$$
\text { ANL-PHY }--85-2
$$

DE 86005582

\title{
DISSIPATIVE HEAVY-ION COLLISIONS
}

\author{
Hans T. Feldmeier
}

Physics Dive: on

Argonne National Laboratory, Argonne, IL 60439

\author{
Dedicated to my wife Renate and my children Elke and Felix \\ who accompanied me during my stay at \\ Argonne National Labcratory
}

\author{
Permanent address: \\ Gesellschaft für Schwerionenforschung mbH \\ Planckstr. 1 , \\ Postfach 110541 \\ D-6100 Darmstadt, West Germany \\ *Heisenberg Fellow
}

\section{DISCLAIMER}

This report was prepared as an account of work sponsored by an agency of the United States Government. Neither the United States Government nor any agency thereof, nor any of their employees, makes any warranty, express or implied, or assumes any legal liability or responsibility for the accuracy, completeness, or usefulness of any information, apparatus, product, or process disclosed, ur represents that its use would not infrigge privately owned rights. Reference herein to any specific commercial product, process, or service by trade name, trademark, manufacturer, or otherwise does not necessarily constitute or imply its endorsement, recommendation, or favoring by the United States Government or any agency thereof. The views and opinions of authors expressed herein do not necessarily state or reflect those of the United States Government or any agency thereof. 
Table of Contents

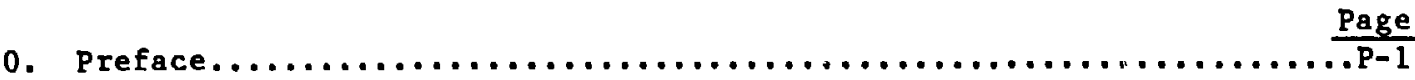

1. Brownian Movement and the Langevin Equation....................

1.1 General Equations of Motion for First and Second Moments.......12

1.2 Conservation I.aws...............................22

2. Master Equation and Fokker-Planck Equation..................27

3. A Model for Dissipative Heavy-Ion Collisions...................31

3.1 Macroscopic variables.................................

$3.2 \quad$ Kinetlc Energy.................................

3.3 Potential Energy $\ldots \ldots \ldots \ldots \ldots \ldots \ldots \ldots \ldots \ldots \ldots \ldots \ldots \ldots \ldots \ldots \ldots \ldots$

3.4 One-body Dissipation.............................

3.4 .1 Wall Dissipation............................

3.4.2 Exchange of Particles, Momentum and Entropy...........62

3.4.3 Window-plus-wa 11 and Wall Dissipation................

3.5 Fluctuations Caused by the Exchange of Particles.............87

3.6 Equations of Motion and Conservation Laws.................

3.7 Results of Trajectory Calculations....................112

4. Dissipative Heavy-1on Collisions................................

4.1 Quasi-elastic and Deep-inelastic Collisions................134

4.2 Large Mass Variance But Small Mass Drift.................141

4.3 Sharing of Excitation Energies.........................

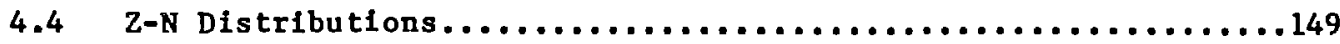

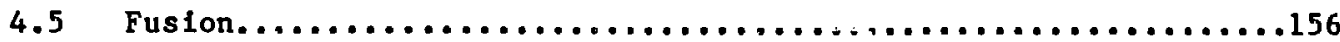

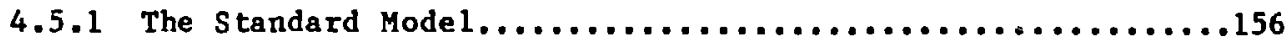

4.5.2 Hindrance in the Fusion of Heavy Nucle1...................

4.6 Dynamics in the Mass Asymmetry..........................

Appendix $\quad \ldots \ldots \ldots \ldots \ldots \ldots \ldots \ldots \ldots \ldots \ldots \ldots \ldots \ldots \ldots \ldots \ldots \ldots \ldots \ldots \ldots \ldots \ldots \ldots \ldots \ldots \ldots \ldots 175$

References............................................. 


$$
\begin{aligned}
& \text { P-1 } \\
& 0 . \quad \text { Preface }
\end{aligned}
$$

These lecture notes comprise a series of lectures held at Argonne National Laboratory In October and November 1984. They may serve as an introduction to dissipative phenomena as observed in collisions of atomic nucle1. These reactions present a formidable task to the theory of nonequilibrium statistical physics. One has to describe a system which has Initially zero temperature and all the energy is residing in kinetic and binding energy. During the colliston which does not last much longer than typical intrinsic times the nuclei become highly excited. Finally, the outgolng fragments or the compound system deexclte again before they are detected in the counters.

In conventional nonequilibrium physics one studies how a system, which is in thermal equilibrium with a large heat bath, responds to a small change in the external parameters like the magnetic field, pressure etc. The situation in heavy-ion collisions is quite different and in a way unique. A closed microscopic system without contact to an external bath evolves irrevers:bly in time from a pure state, prepared by the accelerator, to a mixture as seen by a highly incomplete measurement. From a generalized point of view one may regard all degrees of freedom other than the collective ones as the "heat bath" which absorbs information and thus produces the entropy. However, one should keep in mind that this "heat bath" depends on the choice of the macroscopic variables and on their dynamical evolution. Furthermore, different stages of equilibration may be achieved because the colliding nuclei are pulled apart by electric or centrifugal forces before the macroscopic variables can equilibrate. The great variety of dissipative phenomena seen in 
heavy-ion collisions together with the challenge of statistical physics far from equilibrium has aroused the interest of a whole community of physicists during the last 25 years.

In Section 1 the classical description of Brownian movement is used to introduce the concept of dissipation. The discussion in terms of the Langevin equation contains all basic tdeas which can be found in modern theorles based on the projection formalism. Although the projection formalism is certainly the correct way to introduce friction into a quantum mechanical description the more phenomenological approach is preferred here as it makes the physical concept quite transparent.

In Section 2 the master equation and the Fokker-planck equation are derived. The rextbook of Radu Balescu on "Equilibrlum and Nonequilibrium Statistical Mechanics", John Wiley \& Sons (1975) ISBN-0471-04600-0, may be used for further reference.

Section 3 applies the concepts introduced in Section 1 in a model for disstpative heavy ion collisions. This model is based on the long meanfree-path of nucleons in their selfconsistent one-body potential. Let us call It the "One-body Dissipation Model". Besides the cholce of macroscopic variables and their mutual interaction drift and diffusion coefficients are derived microscopically for different transport processes.

A selection of dissifative phenomena are presented in section 4. Whereever possible the measured data are discussed with help of the one-body dissipation model. 
There were two more lectures which are not covered by these noter. One was on "Scattering Theory and Statistical Properties of the S-matrix" where the link between scattering theory and trajectory calculations was established. Furthermore, it was shown that quantum diffraction effects play only a minor role in dissipative collisions. This lecture followed closely the article by Beck, Spangenberger and Feldmeier published in Nuclear Physics A435 (1985) 267.

The other lecture reviewed time-dependent Hartree-Fock theory, its merits and shortcomings. A comprehensive review can be found in $J$. Negele, Rev. Mod. Phyzics 54 (1982) 913. More results of time dependent Hartree-Fock calculations are contained in the article of Devies, Devi, Koonin and Strayer "TDHF Calculetions of Heavy Ion Collistons" in Heavy Ion Science edited by D. A. Bromley, Plenum Press (1984). 
I wish to thank the members of the Physics Division for their warm hospitality during our stay at Argonne Nattonal Laboratory. I also a:preciate very much the help of Frank Wolfs in preparing the lecture notes and the patience of Donna Nelson, Carol Przyzyck1, and Karen Theyer for typing the many-times revised manuscript. Flnally, I should like to express my gratitude for the continuous ald of Walter Henning and thank Donald S. Gemmell for the invitation. 


\section{Brownian Movement and the Langevin Equation}

The Scottish botanist, Robert Brown, reported in 1827 that an aqueous suspension of pollen (herb clarkla pulchella) contained microscoplc particles, which carried out a continuous haphazard zigzag movement. This random movement was subsequently called "Brownlan Movement". But it took almost 80 years until a satisfactory theoretical description had been given by the contributions of A. Einstein, V. Smoluchowski and P. Langevin (1906).

The study of the Brownian Movement is the key to the understanding of many dissipative processes including such phenomena as friction, noise in an electric circuit, implantation of atoms in metal, diffusion in fluids, or certain heavy-ion reactions,

As Indicated in $\mathrm{fig}$. 1.1 a heavy Brownian particle with mass $\mathrm{M}$ is kicked around by the irregular pushes of the surrounding fluid mclecules with mass $m$. It is assuned that $m$ is much smaller than $M\left(\varepsilon^{2}=\frac{m}{M}<1\right)$.

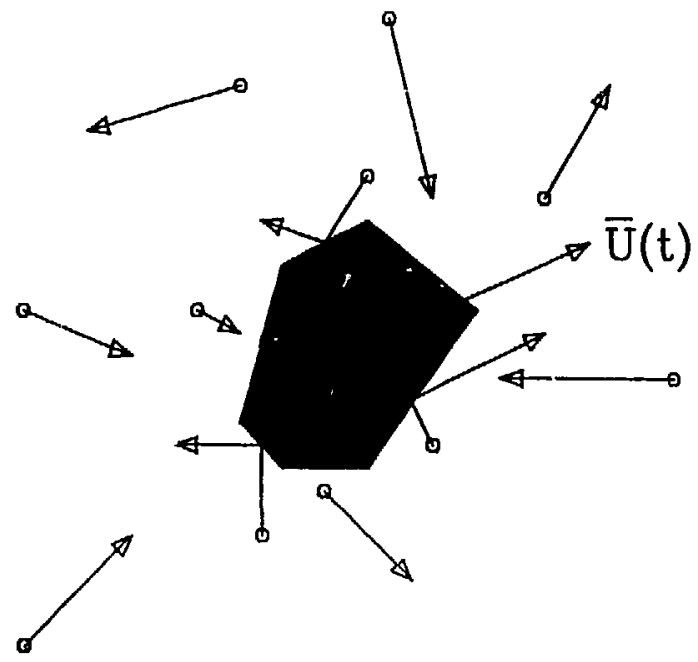

F1g. 1.1

Brownian particle interacts with fluid molecules 
2

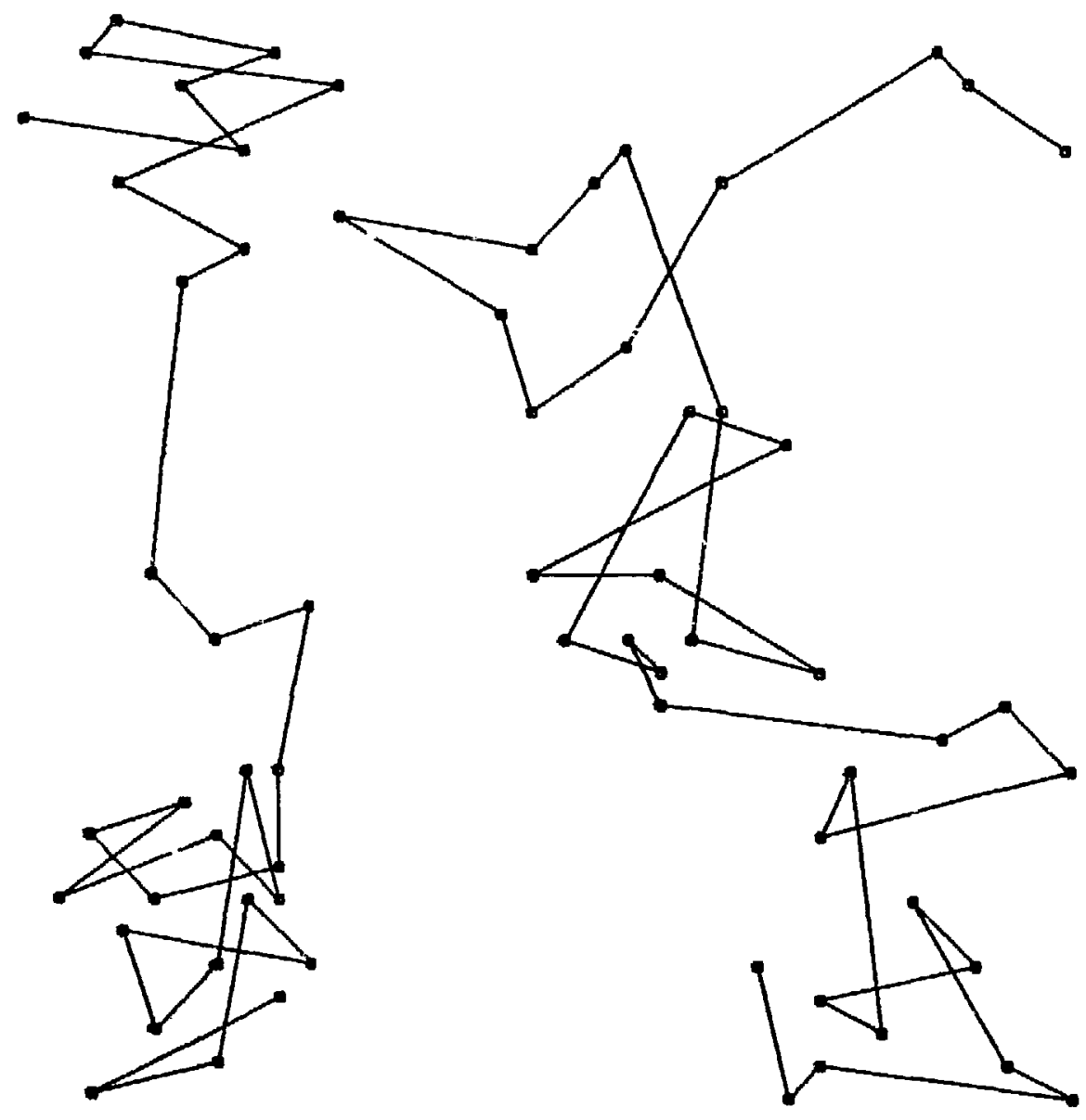

Fig. 1.2 Random movement of a Brownian particle. 
Altogether, a fluctuating force $\vec{X}(t)$ is acting on the particle. The result is a random movement for which no predictions can be made (c.f. fig. 1.2). Nevertheless, a probabilistic treatment of the process is possible and brings order -nto the chaotic motion of a single Brownlan particle. Here I want to present the phenomenological approach [Ch43,WU45] rather than a microscopic derivation [LR63] involving the projection formalism [Gr82]. The fluctuating force $\vec{X}(t)$ can be decomposed into two parts (c.f. fig. 1.3):

$$
\stackrel{\vec{X}}{\mathrm{X}}(\mathrm{t})=\langle\overrightarrow{\mathrm{X}}(\mathrm{t})\rangle+\dot{\delta} \overrightarrow{\mathrm{X}}(\mathrm{t})
$$

where $\leftrightarrow\rangle$ denotes the average over an ensemble of fluids (stochastic average). Experience tells us that in many cases the mean value is proportional to the velocity of the particle:

$$
\langle\vec{X}(t)\rangle=-\gamma \vec{u}(t),
$$

where $\gamma$ is the friction coefficient.

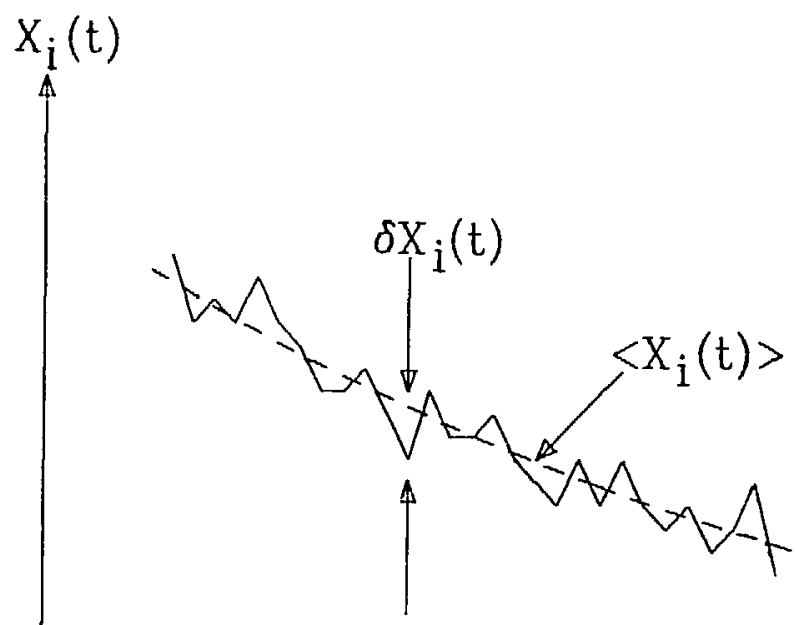

Fig. 1.3 Sketch of a component of the fluctuating force which is decomposed into a mean and a fluctuating part 
The fluctuating force $\delta \vec{F}(t)$ is not known in detall but has the following properties:

$$
\begin{aligned}
& \langle\delta \vec{x}(t)\rangle=0 \\
& \left\langle\delta x_{1}(t) \delta x_{j}(s)\right\rangle=2 D \delta_{1 j} \hat{\delta}(t-8) \text { for } 1, j=1,2,3 .
\end{aligned}
$$

The mean value vanishes by the definition given in eq. (1.1). The autocorrelation in time is supposed to be a sharply peaked function $\hat{\delta}(t-s)$ around $t=s$ (c.f. fig. 1.4). Its characteristic width $\tau_{m}$ is small compared to any macroscopic time scale and resembles the typical time between two successive collisions of the Brownian particle with the fluid molecules. $\tau_{m}$ is called the memory time. The process is called Markovian if $\tau_{\mathrm{m}}<\tau_{\mathrm{coll}}$, where $\tau_{\text {coll }}$ is the typical time for the motion in the collective varlables, here the c.m. motion of the Brownian particle.

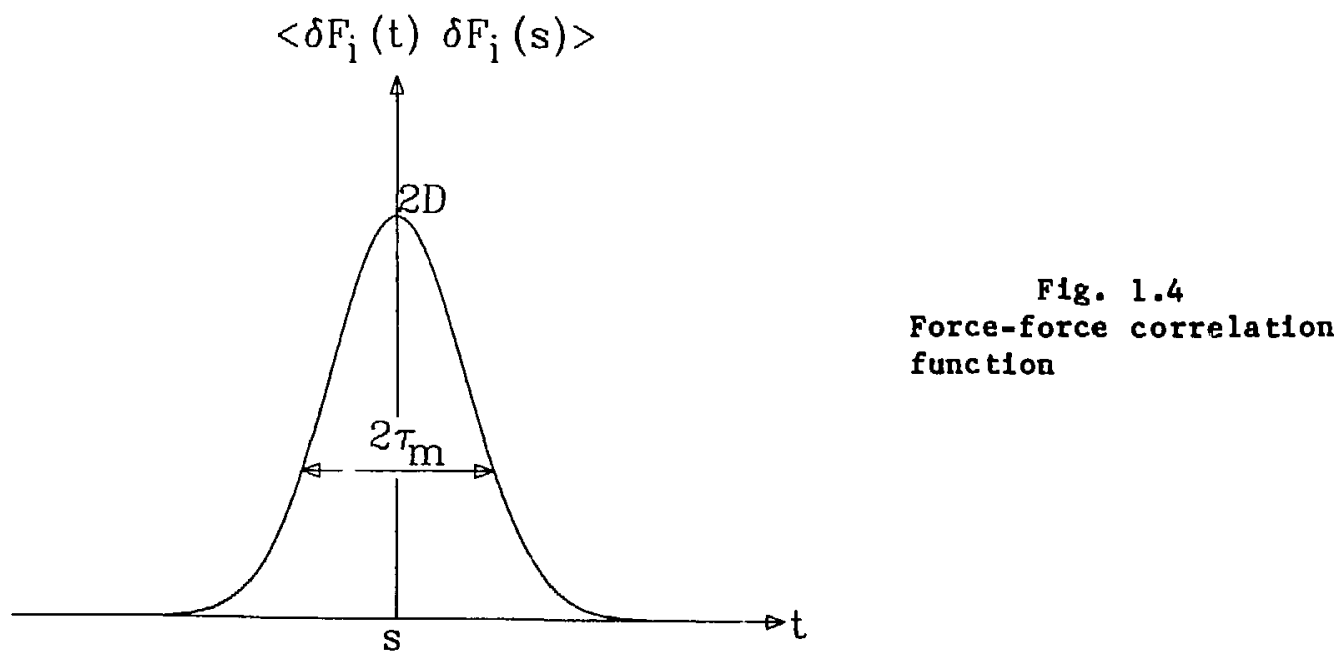

Thus, Integrals of the form $\int f(t, s)\left\langle\delta x_{1}(t) \delta x_{j}(s)\right\rangle d s$ can be evaluated by making the approximation $\hat{\delta}(t-s)+\delta(t-s)$ : 


$$
\int f(t, s)\left\langle\delta x_{1}(t) \delta x_{j}(s)\right\rangle \otimes f(t, t) 2 D \delta_{1 j}
$$

provided $f(t, s)$ varies smoothly in $(t-s)$. The isotropy of the problem implies that $\left\langle\delta x_{1}(t) \delta x_{j}(s)\right\rangle$ Is diagonal and has equal strength in all three spatial directions. This will not be the case for the random exchange of particles between two colliding nuclei as we will see later.

$$
\text { Using ansatz }(1.2),(1.3) \text { and }(1.4) \text { the Brownian movement can be }
$$
described completely. To do this we first write down the equation of motion for the collective variaules of the Brownian particle, which is the so-called Langevin equation:

$$
M \frac{d}{d t} \stackrel{\vec{u}}{u}(t)=-\gamma \vec{u}(t)+\delta \vec{X}(t)
$$

The formal solution of this differential equation is given by

$$
\vec{u}(t)=\vec{u}_{0} e^{-\frac{Y}{M} t}+\frac{1}{M} \int_{0}^{t} e^{-\frac{Y}{M}(t-s)} \delta \vec{X}(s) d s
$$

where $\vec{u}_{0}=\vec{u}(0)$ denotes the initial velocity at time $t=0$. Taking the ensemble average and using eq. (1.3) we obtain an exponentially decaying mean velocity

$$
\langle\vec{u}(t)\rangle=\stackrel{\vec{u}}{0}_{0} e^{-\frac{Y}{M} t}
$$

The mean velocity drops to zero for $t+\infty$. However, the mean kinetic energy should not vanish even if $\langle\vec{u}(t)\rangle=0$ since the particle is still being kicked a round and should possess the thermal kinetic energy accurding to the temperature of the fluid which acts as a heat bath for the Brownian particle. The mean kinetic energy is calculated by squaring eq. (1.7) and averaging 
$\left\langle\frac{M}{2} \vec{u}(t)^{2}\right\rangle=\frac{M}{2} \vec{u}_{0}^{+2} e^{-\frac{2 Y}{M} t}+\frac{1}{2 M} \int_{0}^{t} d s \int_{0}^{t} d s^{\prime} e^{-\frac{Y}{M}\left(2 t-s^{\prime}-s^{\prime}\right)}\left\langle\delta \vec{X}(s) \delta \vec{X}\left(s^{\prime}\right)\right\rangle$

which, due to eq. (1.5), becomes

$$
\left\langle\frac{M}{2} \vec{u}(t)^{2}>=\frac{M}{2} \vec{u}_{0}^{2} e^{-\frac{2 Y}{M} t}+\frac{3}{2} \frac{D}{Y}\left(1-e^{-\frac{2 Y}{M} t}\right)\right.
$$

The initial kinetic energy is damped exponentlally, whereas the thermal energy increases to reach a final value of $\frac{3}{2} \frac{D}{Y}$ for $t+\infty$ (c.f. fig. 1.5). However, we expect this to be equal to $\frac{3}{2} T$, where $T$ is the temperature of the heat bath $\left(k_{B}=\right.$ Boltzmann's constant $\left.=1\right)$. Therefore, we suspect that $\gamma$ and $D$ are related via

$$
T=\frac{D}{Y}
$$
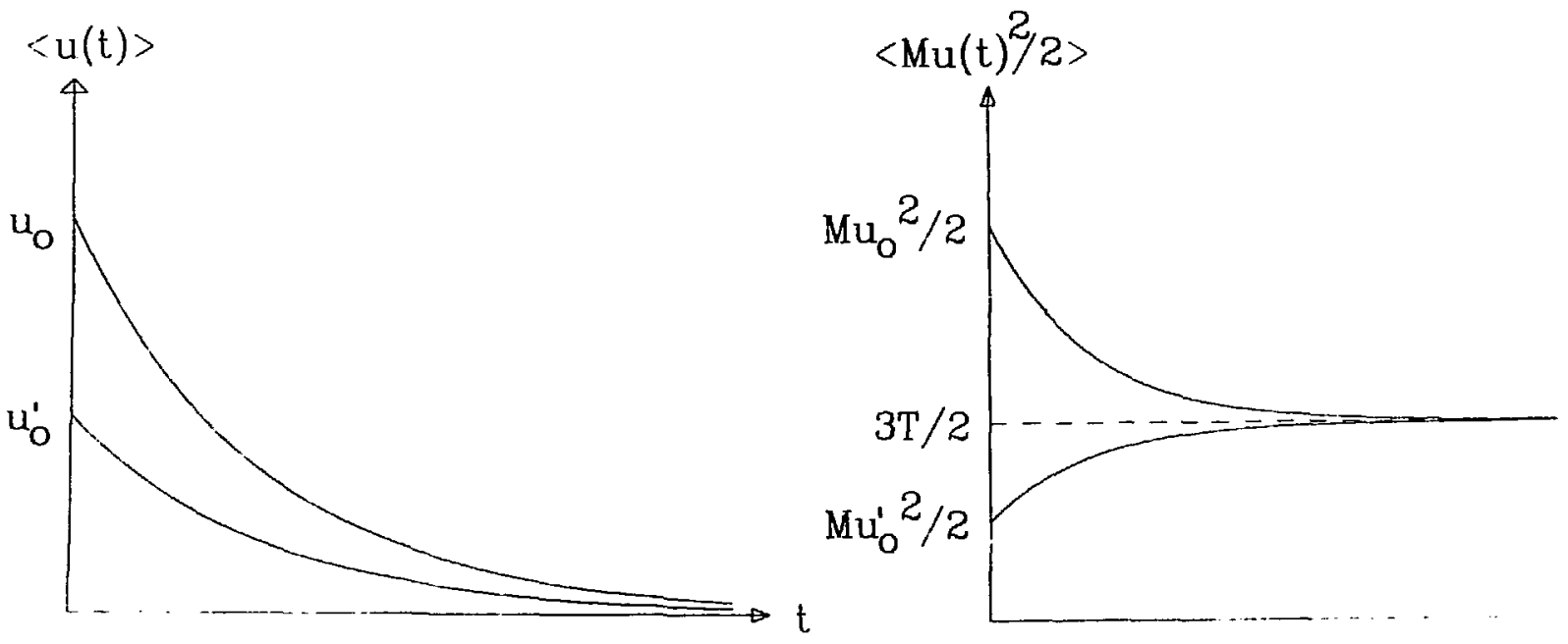

Fig. 1.5

Mean velocity and mean klnetlc energy as a function of time for two different intial conditions 
The ansatz (1.11) can be checked by requiring that the kinetic energy, averaged over a Maxwellian distribution for the initial velocties $\vec{u}_{0}$, should be stationary. Let us denote by a bar the average over a Maxwellian distribution $W_{M}$ :

$$
\overline{f\left(\vec{u}_{0}\right)}=\int d \vec{u}_{0} w_{M}\left(\vec{u}_{0}, T\right) f\left(\vec{u}_{0}\right)
$$

wi th

$$
W_{M}\left(\vec{u}_{0}, T\right)=\left(\frac{M}{2 \pi \mathrm{L}}\right)^{\frac{3}{2}} e^{-\frac{M u_{0}^{2}}{2 T}} .
$$

Averaging eq. (1.10) we obtain

$$
\left.\overline{\left\langle\frac{M}{2} \vec{u}(t)^{2}\right.}\right\rangle=\frac{3}{2} T e^{-\frac{2 \gamma}{M} t}+\frac{3}{2} \frac{D}{\gamma}-\frac{3}{2} \frac{D}{\gamma} e^{-\frac{2 Y}{M} t}
$$

In order to have a stationary $r . n . s$. we have to set $T=D / \gamma$ to get rid of the time dependence. This relation between the friction coefficient $\gamma$, the temperature of the heat bath $T$, and the strength of the fluctuations $D$, represents the most simple form of the so called dissipation-fluctuationtheorem:

$$
\mathrm{D}=\mathrm{T} Y
$$

This equation is also known as the Einstein relation.

Let us now consider the probability distribution for the velocities. $\mathrm{w}_{1}(\vec{u}, t)$ denotes the probability density to find the Brownian particle with a velocity $\vec{u}$ at time $t$. The mere knowledge of $w_{1}(\vec{u}, t)$ is not enough to describe the dynamics of the process, it should rather be the outcome of a diffusion equation. From the joint probability density of finding a value $\vec{u}_{1}$ at time $t$, 
and a value $\vec{u}_{2}$ at $t_{2}$, denoted by $w_{2}\left(\vec{r}_{1}, t_{1} ; \vec{u}_{2}, t_{2}\right)$, we can Infer about the time evolution. However, for a complete characterization of the random process we must, in principle, specify all foint probabilities. It is obvious that these probablity densities must possess the following three properties:

$$
\begin{gathered}
w_{n}>0 \\
w_{m}\left(\vec{u}_{1}, t_{1} ; \vec{u}_{2}, t_{2} ; \ldots ; \vec{u}_{m}, t_{m}\right) \text { 1s symmetric under permutation } \\
\int d^{3} u_{k+1} \ldots d^{3} u_{m} W_{m}\left(\overleftrightarrow{u}_{1}^{+}, t_{1} ; \ldots ; \vec{u}_{m}, t_{m}\right)=w_{k}\left(\stackrel{u}{u}_{1}, t_{1} ; \ldots ; t_{k}, t_{k}\right) \text { for } k<m
\end{gathered}
$$

The Idea behind this classification is that one may concelve of situations in which the knowledge of a few $W_{m}$ is sufficient to characterize the process.

The simplest case in which only $w_{1}$ ls needed and $w_{2}\left(\vec{u}_{1}, t_{1} ; \vec{u}_{2}, t_{2}\right)=$

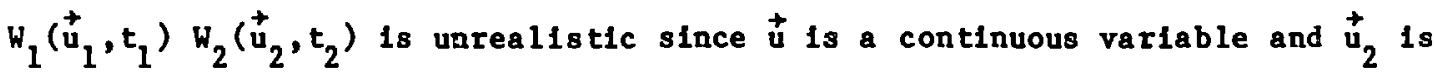
certainly correlated to $\vec{u}_{1}$ if $t_{2}-t_{1}$ is small (if $t_{2}-t_{1}+0$ then $\vec{u}_{2}-\vec{u}_{1}+0$ ).

The next simple case in which the whole information is contained in the first two functions $w_{1}$ and $w_{2}$ is of fundamental importance in statistical physics; it is called the Markovian process.

It is convenient to introduce the conditional probability $w_{2}\left(\vec{u}_{2}, t_{2} \mid \vec{u}_{1}, t_{1}\right)$ defined as

$$
w_{2}\left(\vec{u}_{2}, t_{2} \mid \vec{u}_{1}, t_{1}\right)=\frac{w_{2}\left(\vec{u}_{1}, t_{1} ; \vec{u}_{2}, t_{2}\right)}{w_{1}\left(\vec{u}_{1}, t_{1}\right)}
$$

which is the probability to find the velocity $\vec{u}_{2}$ at time $t_{2}$ provided the Browntan parifle had a velocity $\vec{u}_{1}$ at time $t_{1}$. The relations (1.16) 1mply the following properties for $w_{2}$ 


$$
\begin{aligned}
& w_{2}\left(\vec{u}_{2}, t_{2} \mid \vec{u}_{1}, t_{1}\right)>0 \\
& \int d^{3} u_{2} w_{2}\left(\vec{u}_{2}, t_{2} \mid \vec{u}_{1}, t_{1}\right)=1 \\
& w_{1}\left(\vec{u}_{2}, t_{2}\right)=\int d^{3} u_{1} w_{2}\left(\vec{u}_{2}, t_{2} \mid \vec{u}_{1} t_{1}\right) w_{1}\left(\vec{u}_{1}, t_{1}\right) \text { for } t_{2}>t_{1}
\end{aligned}
$$

The last Integral equation shows the property of $W_{2}$ being a transition probability. The distribution at a time $t_{2}$ can be calculated from the knowledge of the distribution $w_{1}$ at time $t_{1}$ and the transition probability to go from a given $\vec{u}_{1}$ to all possible $\vec{u}_{2}$ within the time interval $t_{2}-t_{1}$. Let us consider the n-th order tansition probability

$$
w_{n}\left(\vec{u}_{n}, t_{n}\left|\vec{u}_{n-1} t_{n-1}\right| \cdots \mid \vec{u}_{1}, t_{1}\right)
$$

which is defined as the conditional probability density of finding the velocity $\vec{u}_{n}$ at time $t_{n}$ provided the system had the velocity $\vec{u}_{n-1}$ at time $t_{n-1}$ and so on for times $t_{n}>t_{n-1}>\ldots>t_{1}$. Herewith, one can define a Markovian process by the condition

$$
w_{n}\left(\vec{u}_{n}, t_{n}|\cdots| \vec{u}_{1}, t_{1}\right)=w_{2}\left(\vec{u}_{n} t_{n} \mid \vec{u}_{n-1}, t_{n-1}\right)
$$

This equation implies that the probability of a transition at time $t_{n-1}$ to time $t_{n}$ depends only on the value of $\vec{u}_{\text {at }}$ time $t_{n-1}$ and not on the previous history of the system.

It is easy to check that for a Markovian process all joint probability densities $W_{n}$ for $n>3$ can be expressed in terms of $W_{1}$ and $W_{2}$. Furthermore, one can show that condition (1.21) implies 


$$
w_{2}\left(\vec{u}_{3}, t_{3} \mid \vec{u}_{1}, t_{1}\right)=\int d^{3} u_{2} w_{2}\left(\vec{u}_{3}, t_{3} \mid \vec{u}_{2} t_{2}\right) w_{2}\left(\vec{u}_{2}, t_{2} \mid \vec{u}_{1}, t_{1}\right)
$$

This important integral equation, obeyed by the transition probability, is of ten taken as the definition of a Markovian process. It is called the Chapman-Kolmogorov equation or, sometimes, the Smoluchowsk1 equation.

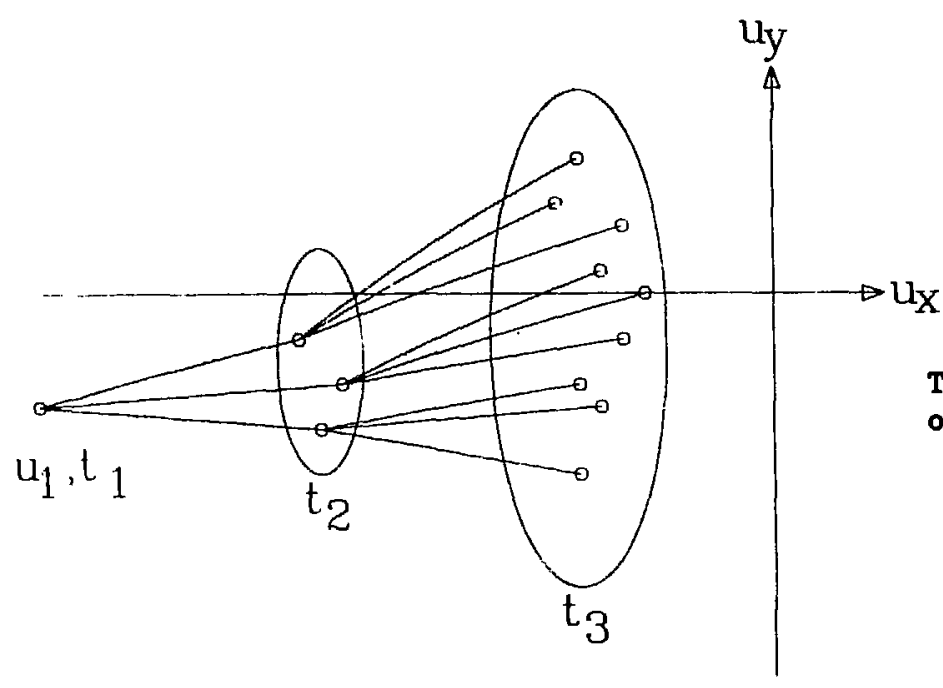

Fig. 1.6

The physical interpretation of eq. $(1.22)$

Figure 1.6 illustrates the Markovian diffusion in velocity space as expressed In eq. (1.22). A particle which at time $t_{1}$ has velocity $u_{1}$ assumes a velocity distribution at time $t_{2}\left(t_{2}>t_{1}\right)$ which has a mean value different from $u_{1}$ and is spread out. Later at time $t_{3}$ the distribution has broadened further and the mean value approaches $\vec{u}_{0} 0$. By successive folding of the previous distribution one describes the dissipative time evolution. 
The conditional probability $w_{2}$ for the Brownian particle turns out to be

$W_{2}\left(\vec{u}_{2}, t_{2} \mid \vec{u}_{1} t_{1}\right)=\left[\frac{M / 2 \pi T}{1-\exp \left(-2 \frac{Y}{M}\left|t_{2}-t_{1}\right|\right)}\right]^{\frac{3}{2}} \exp \left(-\frac{M}{2 T} \frac{\left(\vec{u}_{2}-\vec{u}_{1} \exp \left(-\frac{Y}{M}\left|t_{2}-t_{1}\right|\right)\right)^{2}}{1-\exp \left(-\frac{2 Y}{M}\left|t_{2}-t_{1}\right|\right)}\right)$

One sees that for $t_{2}-t_{1}+\infty$ the distribution ends up as a Maxwell distribution independently of the initial velocity $\stackrel{t}{u}_{1}$. For $t_{2}-t_{1}+0$ it becomes sharply peaked around $\vec{u}_{2}-\vec{u}_{1}$ and approaches $\delta\left(\vec{u}_{2}-\vec{u}_{1}\right)$. 


\subsection{General equations of mecion for first and second moments}

In this subsection we derive the equations of motion for the mean values of the macroscopic variables and their second moments. We follow the ideas employed to describe Brownian motion [Re65, FS85], where one starts with the most general form of the Langevin equation:

$$
\dot{\mathrm{p}}_{1}(t)=k_{1}(\stackrel{+}{\mathrm{p}}(t), \vec{q}(t))+x_{1}(t) ; 1=1, \ldots, n
$$

and the relation between the velocities and momenta:

$$
\dot{q}_{1}(t)=\sum_{k} M^{-1}(\vec{q}(t)){ }_{1 k} p_{k}(t) ; 1=1, \ldots, n .
$$

Here, $K_{1}(\stackrel{+}{p}, \vec{q})$ de:s.:s the forces acting between the macroscopic variables $\left(K_{i}=-\frac{\partial H}{\partial q_{1}}, H=\right.$ Hamiltonian) while $x_{1}(t)$ are the forces originating from the intrinsic degrees of freedom which produce an irregular distortion of the macroscopic momenta. The inverse mass tensor, $M^{-1}\left(q_{q}^{+}\right)$, may be coordinate dependent.

Next one supposes that the system can be described by a phase-space distribution $f_{\text {tot }}(\vec{p}, \vec{q}, \xi, t)$, which includes all the macroscopic and intrinsic degrees of freedom. A reduced phase-space distribution, $f(\vec{p}, \vec{q}, t)$, for the macroscopic variables alone is obtained by integrating over the intrinsic variables:

$$
f(\vec{p}, \stackrel{+}{q}, t)=\int d^{m} \xi f_{t c t}(\stackrel{+}{p}, \stackrel{+}{q}, \xi, t)
$$

The phase-space distribution will not be specified explicitly but serves only to define averaged quantities. It is assumed to be normalized. 
The mean valuea of the macroscoplc varfables are denoted by capital

letters:

$$
\begin{aligned}
& P_{1}(t)=\left\langle p_{1}(t)\right\rangle=\int d^{n} p d^{n} q f\left(p_{1}^{+},+t\right) p_{1} \\
& Q_{1}(t)=\left\langle q_{1}(t)\right\rangle=\int d^{n} p d^{n} q f\left(\stackrel{p}{+},+_{q}^{+}, t\right) q_{1}
\end{aligned}
$$

It should be noted that in the preceding equations the letters $p_{1}$ and $q_{1}$ are Integration variables, whereas $p_{i}(t)$ and $q_{i}(t)$ are solutions of the Langevin equation (eq. $(1.1 .1)$ ) and of eq. (1.1.2). respectively. The momentum $P_{1}(t+\tau)$ at a time $\tau$ later than $t$ may be written in two ways:

$$
\begin{aligned}
& P_{1}(t+\tau)=\left\langle p_{1}(t+\tau)\right\rangle=\int d^{n} p d^{n} q f(\vec{p}, \vec{q}, t+\tau) p_{1} \\
& P_{1}(t+\tau)=\int d^{n} p d^{n} q d^{m} \xi f_{t o t}(\vec{p}, \vec{q}, \xi, t) \hat{p}_{1}(t+\tau) .
\end{aligned}
$$

In eq. (1.1.7) $\hat{p}_{1}(t+\tau)$ is a solution of the Langevin equation with the initial condition $\hat{p}_{1}(t)=p_{1}$, where $p_{1}$ is the integration variable. Hence, through the action of $x_{1}(t)$ the momentum $\hat{p}_{1}(t+\tau)$ depends also on $\xi$ and, therefore, $f_{\text {tot }}\left(\stackrel{+}{p}, q^{+}, \xi, t\right)$ has to be used. Furthermore, $\hat{p}_{1}(t+\tau)$ depends on the initial condition $\hat{p}_{1}(t)=p_{1}$, so that for every incagration point $(\stackrel{+}{p}, \vec{q})$ in phase space one has to solve the equations of motion (eqs. $(1.1 .1$ ) and $(1.1 .2)$ ) with the appropriate initial conditions.

To achleve macroscopic equations of motion [Ch43, K146] one considers first the time evolution during a time interval $\tau$, which is short on the macroscopic time scale but long on the microscopic scale: 


$$
\begin{aligned}
& p_{1}(t+\tau)=p_{i}(t)+\int_{t}^{t+\tau} d t K_{i}^{\prime}\left(\vec{p}\left(t^{\prime}\right), \vec{q}\left(t^{\prime}\right)\right)+\int_{t}^{t+\tau} d t^{\prime} x\left(t^{\prime}\right) \\
& q_{1}(t+\tau)=q_{1}(t)+\sum_{k}^{t+\tau} d t^{\prime} M^{-1}\left(q^{+}\left(t^{\prime}\right)\right){ }_{1 k^{2}} p_{k}\left(t^{\prime}\right)
\end{aligned}
$$

The terminology of macroscopic and microscopic time scales implies the 1at assumption: that 1 it is possible to choose a set of macroscopic variables which vary slowly in time such that the remaining intrinsic degrees of freedom possess much higher frequences. After taking averages of eqs. (1.1.8) and (1.1.9) with the phase-spacedistribution at time $t$ one employs the

2nd assumption: that the phase-space distribution $f(\vec{p}, \vec{q}, t)$, is peaked sharply around the mean values.

Actually, it has to be localized enough to allow for the following approximations:

$$
\begin{aligned}
& \left\langle K_{i}(\vec{p}(t), \vec{q}(t))\right\rangle=K_{i}(\vec{P}(t), \vec{Q}(t)) \\
& \left\langle M^{-1}(\vec{q}(t))_{1 k_{k}} P_{k}(t)\right\rangle \approx M^{-1}(\stackrel{Q}{Q}(t))_{1 k} P_{k}(t) .
\end{aligned}
$$

One can extend the validity of these approximations to broader distributions by expanding around the mean value and keeping terms up to first derivatives in the mass tensor or second derivatives for the potential $V(q)$. This would also be more consistent with the equations of motion for the variances $(1.1 .31)-(1.1 .33)$. If one includes the effect of the variances in the equations for the mean values eqs. $(1.1 .10)$ and $(1.1 .11)$ are to be replaced by 


$$
\begin{aligned}
& \left\langle K_{1}(\stackrel{+}{P}(t), \vec{q}(t)\rangle \simeq K_{1}(\vec{P}(t), \vec{Q}(t))-\frac{1}{2} \sum_{k 1} \frac{\partial M_{k 1}^{-1}}{\partial q_{1}}(\vec{Q}(t)) A_{k 1}(t)\right. \\
& \left\langle M^{-1}(\vec{q}(t))_{1 k} \vec{P}_{k}(t)\right\rangle=M^{-1}(\vec{Q}(t))_{1 k} \vec{P}_{k}(t)+\sum_{1}^{T} \frac{\partial \Gamma_{1 k}^{-1}}{\partial q_{1}}(\vec{Q}(t)) C_{k 1}(t)
\end{aligned}
$$

whe:s: we assume tine Hamlltonfan to be of the form $\left.H_{1}^{+},+\vec{q}\right)=\frac{1}{2} \sum_{1 k} M^{-1}(\vec{q}){ }_{1 k} P_{1} P_{k}+V(\vec{q})$. For the definitions of $A_{k 1}$ and $C_{k 1}(t)$ see eqs. (1.1.22) and (1.1.24).

Since $\tau$ 1s supposed to be small on the macroscopic scale one keeps only terms which are of lowest order in $\tau$ :

$$
\begin{aligned}
& \left\langle\int_{t}^{t+\tau} d t^{\prime} K_{i}\left(\vec{p}\left(t^{\prime}\right),+q^{+}\left(t^{\prime}\right)\right)\right\rangle \simeq \tau K_{1}(\vec{P}(t), \stackrel{Q}{Q}(t)) \\
& \left\langle\int_{t}^{t+\tau} d t^{\prime} M^{-1}\left(q^{+}\left(t^{\prime}\right)\right){ }_{1 k} P_{k}\left(t^{\prime}\right)\right\rangle=\tau M^{-1}\left(Q^{+}(t)\right){ }_{1 k} P_{k}(t) .
\end{aligned}
$$

Thus, one gets for the change in the macroscopic varlables during time $\tau$ the following expressions:

$$
\begin{aligned}
& \frac{P_{1}(t+\tau)-P_{1}(t)}{\tau}=K_{1}(\vec{P}(t), \vec{Q}(t))+\frac{1}{\tau}\left\langle\int_{t}^{t+\tau} d t^{\prime} X_{1}\left(t^{\prime}\right)\right\rangle \\
& \frac{Q_{1}(t+\tau)-Q_{1}(t)}{\tau}=\sum_{k} M^{-1}\left(Q^{+}(t)\right){ }_{1 k} P_{k}(t) .
\end{aligned}
$$

Here, the left hand side is regarded as a time derivative. However, $\tau$ cannot go to zero because the treatment of the averaged value of the fluctuating force (seconds term on r.h.s. of eq. (1.1.14)) requires a finite $\tau$. One can only specify general properties of $x_{1}(t)$ since it is not known in detall. Let us denote by $n_{1}$ the change of the momentum during the time $\tau$ caused by the Irregular actions of the Internal degrees of freedom: 


$$
n_{1}=\int_{t}^{t+\tau} d t^{\prime} x_{1}\left(t^{\prime}\right)
$$

One presumes that the information known about $X_{1}(t)$ is a probability distribution $w(\vec{p}, \vec{q}, \mid \vec{n}, \tau)$ for the random variable $\vec{n}$ (the analogue to $W_{2}\left(\vec{u}_{2} t_{2} \mid \vec{u}_{1} t_{1}\right)$ defined in section 1$)$. $W(\vec{p}, \vec{q}, \mid \vec{n}, \tau) d^{n} n$ denotes the probability of finding the system with momentum $\vec{p}+\vec{n}$ at time $t+\tau$ provided it resided at $\vec{p}$ and $\vec{q}$ in a phase space at time $t$. By not specifying $t$ explicity in the 11st of agruments one made use of the

3rd assumption: that the system behaves in a Markovian way. The actions of the internal degrees of freedom at time t+ $\tau$ is uncorrelated to the one at time $t$, where $\tau$ is a time during which the macroscopic varlables have changed by negliglble amounts.

In subsection 3.5 we shall derive an explicit expression for $w(\vec{p}, \vec{q}, \mid \vec{\eta}, \tau)$ by assuming a statistical exchange of nucleons between the two nuclef. Returning now to the equations of motion, the friction force is given by

$$
\begin{aligned}
& \frac{1}{\tau}<\int_{t}^{t+\tau} d t^{\prime} x_{i}\left(t^{\prime}\right)>=\frac{1}{\tau} \int d^{n} p d^{n} q f(\vec{p}, \stackrel{+}{q}, t) \int d^{n} n w(\stackrel{+}{p}, \stackrel{+}{q}, \mid \vec{n}, \tau) \eta_{1} \\
& \simeq \frac{1}{\tau} \int d^{n} \eta W(\vec{P}(t), \vec{Q}(t) \mid \vec{n}, \tau) \eta_{1} \\
& =F_{1}((\stackrel{+}{P}(t), \stackrel{+}{Q}(t)) \text {. }
\end{aligned}
$$

In order to get the last line of eq. (1.1.17) one makes use of the 2 nd assumption by claiming that $W(\vec{p}, \vec{q}, \mid \vec{n}, \tau)$ depends weakly on $\vec{p}$ and $\vec{q}$. If the resulting friction force 18 of the type $F_{1}(\vec{p}, \vec{q})=-\sum_{t} R_{1 k}(\vec{q}) \vec{p}_{k}$ the inclusion of higher order terms leads to 
$\left\langle\int_{t}^{t+\tau} d t^{\prime} x_{1}\left(t^{\prime}\right)\right\rangle=F_{i}(\stackrel{+}{P}(t), \stackrel{+}{Q}(t))-\sum_{k 1} \frac{\partial R_{1 k}}{\partial q_{1}}(\stackrel{+}{Q}(t)) c_{k 1}(t)$

Neglecting the higher order terms, one obtains the following equations of motion for the first moments:

$$
\begin{aligned}
& \frac{d}{d t} F_{1}(t)=K_{1}(\stackrel{+}{P}(t), \stackrel{+}{Q}(t))+F_{1}(\stackrel{+}{P}(t), \stackrel{+}{Q}(t)) ; 1=1, \ldots, n \\
& \frac{d}{d t} Q_{1}(t)=\sum_{k} M^{-1}(\stackrel{+}{Q}(t))_{1 k} P_{k}(t) ; 1=1, \ldots, n .
\end{aligned}
$$

The mass tensor, $M_{1 k}$, and conservative forces, $K_{1}$, will be defined in subsection 3.2 and 3.3 , respectively, while the friction force will be calculated in subsection 3.4 .

Let the deviations from the mean values be denoted by:

$$
\begin{aligned}
& \Delta p_{1}(t)=p_{1}(t)-p_{i}(t) \\
& \Delta q_{i}(t)=q_{i}(t)-Q_{i}(t) .
\end{aligned}
$$

Then the variances or second moments of the macroscopic variables are defined as:

$$
\begin{aligned}
& A_{i j}(t)=\left\langle\Delta p_{1}(t) \Delta p_{j}(t)\right\rangle \\
& B_{i j}(t)=\left\langle\Delta q_{i}(t) \Delta q_{j}(t)\right\rangle \\
& C_{1 j}(t)=\left\langle\Delta p_{i}(t) \Delta q_{j}(t)\right\rangle .
\end{aligned}
$$


To derive the macroscopic time derivatives of the variances one first calculates $A_{1 j}(t+\tau), B_{1 j}(t+\tau)$ and $C_{1 j}(t+\tau)$ by subtracting from eq. (1.1.8) its mean value:

$$
\begin{aligned}
\Delta \mathrm{p}_{1}(t+\tau)=\Delta \mathrm{p}_{1}(t)+\tau\left\{K_{1}(\vec{p}(t), \vec{q}(t))-k_{1}(\vec{P}(t), \vec{Q}(t))\right\} \\
\quad+\int_{t}^{t+\tau} d t^{\prime} x_{i}\left(t^{\prime}\right)-\tau F_{1}(\vec{P}(t), \vec{Q}(t)) .
\end{aligned}
$$

Since the phase-space distribution is peaked sharply around the mean values, the expression in the curly brackets of eq. (1.1.25) may be expanded up to linear terms:

$$
\mathrm{K}_{1}(\overrightarrow{\mathrm{p}}, \overrightarrow{\mathrm{q}})-\mathrm{K}_{\mathrm{i}}(\overrightarrow{\mathrm{P}}, \overrightarrow{\mathrm{Q}}) \simeq \sum_{\mathrm{k}} \Delta \mathrm{p}_{\mathrm{k}} \frac{\partial \mathrm{K}_{1}}{\partial \mathrm{p}_{\mathrm{k}}}(\overrightarrow{\mathrm{P}}, \overrightarrow{\mathrm{Q}})+\sum_{\mathrm{k}} \Delta \mathrm{q}_{\mathrm{k}} \frac{\partial \mathrm{K}_{1}}{\partial \mathrm{q}_{\mathrm{k}}}(\overrightarrow{\mathrm{P}}, \overrightarrow{\mathrm{Q}})
$$

By muiciplying eq. $(1,1.25)$ with itself, but with the subscript changed to $j$, and taking the average of the result, one encounters the two following terms which will be discussed separately. Firstly,

$$
\begin{aligned}
& \left\langle\Delta p_{i}(t)\left\{\int_{t}^{t+\tau} d t^{\prime} x_{j}\left(t^{\prime}\right)-\tau F_{j}(\vec{P}(t), \vec{Q}(t))\right\}\right\rangle \\
& =\int d^{n} p^{n}{ }_{q} f(\vec{p}, \vec{q}, t) \Delta p_{1} \int d^{n} n w(\vec{p}, \vec{q} \mid \vec{n}, \tau) \eta_{j} \\
& =\tau \sum_{k}\left\{A_{i k}(t) \frac{\partial F}{\partial p_{k}}(\vec{P}, \vec{Q})+C_{i k}(t) \frac{\partial F}{\partial q_{k}}(\vec{P}, \vec{Q})\right\},
\end{aligned}
$$

where the part with $\tau F_{j}$ vanishes because $\left\langle\Delta p_{1}(t)\right\rangle=0$. Since $w(\vec{p}, \vec{q} \mid \vec{n}, \tau)$ is supposed to depend weakly on the first two arguments and the phase-space distribution is narrow, one expands around the mean values and uses eq. 
(1.1.17) together with the normalization condition to derive the third line of eq. (1.1.27). Secondly,

$$
\left\langle\int_{t}^{t+\tau} d t^{\prime} x_{i}\left(t^{\prime}\right) \int_{t}^{t+\tau} d t^{\prime \prime} x_{j}\left(t^{\prime \prime}\right)\right\rangle=\int d^{n} n W\left(\stackrel{+}{P}(t), \stackrel{+}{Q}(t)||_{n}^{+}, \tau\right) n_{i} n_{j} \cdot
$$

This term is the source of the diffusion of the relative momentic originating In irregular "kicks" from the intrinsic degrees of freedom. The diffusion tensor, $D_{1 j}$, is defined as the second moment of the previously introduced conditional probability $w(\vec{p}, \vec{q} \mid \vec{n}, \tau)$, divided by $2 \tau$ :

$$
D_{1 j}(t)=\frac{1}{\tau} \frac{1}{2} \int d^{n} \eta W(\stackrel{P}{P}(t), \stackrel{Q}{Q}(t) \mid \stackrel{+}{n}, \tau) n_{1} n_{j} \cdot
$$

It depends on the mean trajectory $\{\vec{P}(t), \dot{Q}(t)\}$ and is time dependent. Looking back at eq. (1.1.17) one sees that the friction force turned out to be the first moment of $w(\vec{p}, \vec{q} \mid \vec{n}, \tau)$ with respect to $\vec{n}$, divided by $\tau$. Equations $(1.1 .17)$ and $(1.1 .29)$ resemble the so called dissipation-fluctuation relation. Here, it is presented in its most general form. The Einstein relation is a special case where the intrinsic degrees of freedom can be describea by just one parameter, namely, the temperature. This will not be the case for nucleus-nucleus collisions in the particle exchange model as derived in subsection 3.5 .

By collecting all terms and neglecting those which are of order $\tau^{2}$ one gets:

$$
\begin{aligned}
\left\langle\Delta p_{i}(t+\tau) \Delta p_{j}(t+\tau)\right\rangle=\left\langle\Delta p_{1}(t) \Delta p_{j}(t)\right\rangle \\
+\tau \sum_{k}\left\{A_{j k}(t) \frac{\partial\left(K_{1}+F_{1}\right)}{\partial P_{k}}+A_{i k}(t) \frac{\partial\left(K_{j}+F_{j}\right)}{\partial P_{k}}\right\}
\end{aligned}
$$




$$
\begin{aligned}
& +\tau \sum_{k}\left\{C_{j k}(t) \frac{\partial\left(K_{1}+F_{1}\right)}{\partial Q_{k}}+c_{1 k}(t) \frac{\partial\left(K_{j}+F_{j}\right)}{\partial Q_{k}}\right\} \\
& +2 \tau D_{i j}(t) .
\end{aligned}
$$

Simflar reasoning leads to the macroscople time derlvative for $B_{1 j}(t)$ and $c_{1 j}(t)$. The results are summarized in eqs. $(1.1 .31),(1.1 .32)$ and $(1.1 .33)$

$$
\begin{aligned}
\frac{d}{d t} A_{1 j}(t) & =\frac{1}{\tau}\left[A_{1 j}(t+\tau)-A_{1 j}(t)\right\}= \\
& \sum_{k}\left\{A_{j k}(t) \frac{\partial\left(K_{1}+F_{1}\right)}{\partial P_{k}}+A_{1 k}(t) \frac{\partial\left(K_{j}+F_{j}\right)}{\partial Q_{k}}\right\} \\
& +\sum_{k}\left\{C_{j k}(t) \frac{\partial\left(K_{1}+F_{1}\right)}{\partial Q_{k}}+C_{1 k}(t) \frac{\partial\left(K_{j}+F_{j}\right)}{\partial Q_{k}}\right\} \\
+ & 2 D_{1 j}(t)
\end{aligned}
$$$$
\frac{d}{d t} B_{1 j}(t)=\frac{1}{\tau}\left\{B_{1 j}(t+\tau)-B_{1 j}(t)\right\}=
$$$$
\sum_{k}\left\{C_{k i}(t) M_{j k}^{-1}+C_{k j}(t) M_{1 k}^{-1}\right\}
$$$$
+\sum_{k, 1}\left[B_{j k}(t) \frac{\partial M_{i 1}^{-1}}{\partial Q_{k}}+B_{i k}(t) \frac{\partial M_{j 1}^{-1}}{\partial Q_{k}}\right] P_{1}(t)
$$

$$
\begin{aligned}
\frac{d}{d t} C_{1 j}(t)= & \frac{1}{\tau}\left\{C_{1 j}(t+\tau)-C_{1 j}(t)\right\}= \\
& \sum_{k}\left\{B_{j k}(t) \frac{\partial\left(K_{1}+F_{1}\right)}{\partial Q_{k}}+C_{k j}(t) \frac{\partial\left(K_{1}+F_{1}\right)}{\partial P_{k}}\right\}
\end{aligned}
$$




$$
+\sum_{k} A_{1 k}(t) M_{j k}^{-1}+\sum_{k, 1} C_{i k}(t) \frac{\partial M_{j 1}^{-1}}{\partial Q_{k}} P_{1}(t)
$$

In these equations all quantities which depend on the macroscopic variables have to be taken at the mean values which the system has at time $t$.

A moment expansion of the Fokker-Planck equation [HS77] which w111 be derived and discussed in the following section yields the same equations. The derivation shown here, however, does not need the detour in going from the Lasgevin equation to the Master equation from there to the Fokker-Planck equation and then to the moment expansion. 


\subsection{Conservation laws}

The dynamics of the macroscopic degrees of freedom as well as the interaction with the intrinsic degrees of freedom is in general obeying conservation laws. For example, in a heavy ion collision which may be regarded as a micro-canonical system a model description should take care of the conservation of total energy, total momentum, total spin, total particle number, etc. These conservation laws induce correlations in the mean values and varlances.

Let us denote a conservation law by

$$
c_{a}(y)=0
$$

where $y=\left\{y_{1} ; 1=1, \ldots, n\right\}$ stands for the macroscopic variables. If the action of the intrinisic degrees of freedom which is described by the conditional probability $W_{2}\left(y^{\prime}, t^{\prime} \mid y, t\right)$ obey the conservation law $(1.2 .1)$ and if $c_{a}(y)=0$ then also $C_{a}\left(y^{\prime}\right)=0$. For sufficiently small time intervals $t^{\prime}-t$ we may expand

$$
c_{a}\left(y^{\prime}\right)=c_{a}(y)+\sum_{k} \frac{\partial c_{a}(y)}{\partial y_{k}}\left(y_{k}^{\prime}-y_{k}\right)+\ldots=0
$$

If $y$ is in accord with the conservation law $\left(C_{a}(y)=0\right)$ we deduce from eq. $(1.2 .2$.$) with n=y^{\prime}-y$

$$
\lim _{t^{\prime} \rightarrow t} \frac{1}{t^{\prime}-t} \int d^{n} n \sum w_{2}\left(y+n, t \mid y, t^{\prime}\right) \sum_{k} \frac{\partial C_{a}}{\partial y_{k}}(y) \eta_{k} n_{1}=0
$$

This provides a relation between the different diffusion coeffictents 


$$
\sum_{k} \frac{\partial C_{a}}{\partial y_{k}} D_{k l}=0
$$

Here we used the definition $(1.1 .29)$ for the diffusion coefficients. For the drift coefficients which are the first moments of $w_{2}$ one has to invoke the conservative forces as well.

Similar relations can be derived for first and second moments of the phase space distribution. Let us always presume that the distribution at on initial time obeyed the conservation laws. With the assumption of a narrowly peaked phase space distribution one may expand around the mean values

$$
c_{a}(\langle y\rangle)+\left.\sum_{1} \frac{\partial c_{a}}{\partial y_{i}}\right|_{\langle y\rangle} \Delta y_{1}+\left.\frac{1}{2} \sum_{1 j} \frac{\partial^{2} c_{a}}{\partial y_{i} \partial y_{j}}\right|_{\langle y\rangle} \Delta y_{i} \Delta y_{j}+. \cdot \cdot
$$

where $\Delta y_{1}=y_{1}-\left\langle y_{1}\right\rangle$ denotes the deviation from the mean value. If the conservation law is linear in $y$ eq. (1.2.5) implies the conservation law for the mean values

$$
C_{a}(\langle y\rangle)=0
$$

and the exact relations among the variances

$$
\left.\sum_{1} \frac{\partial C_{a}}{\partial y_{1}}\right|_{\langle y\rangle} A_{1 k}=0 \quad \text { for all } k
$$

where $A_{1 k}=\left\langle\Delta y_{1} \Delta y_{k}\right\rangle$.

If the conservation law is quadratic the expectation value of eq. $(1.2 .2$.$) provides the following exact relation$

$$
c_{a}(\langle y\rangle)+\left.\frac{1}{2} \sum_{i j} \frac{\partial^{2} c_{a}}{\partial y_{1} \partial y_{j}}\right|_{\langle y\rangle} A_{1 j}=0
$$


For conservation laws whych are of higher order in $y$ eqs. (1.2.7) and $(1.2 .8)$ ar fulfilled only approsimately, the narrower the distribution the better the accuracy.

Let us consider three examples. First, a linear conservation law between two observables like particle number or momentum:

$$
C\left(y_{1}, y_{2}\right)=y_{1}+y_{2}-A=0
$$

Eq. $(1.2 .3)$ becomes

$$
\sigma_{11}+\sigma_{12}=\sigma_{22}+\sigma_{21}=0
$$

or since $\sigma_{12}=\sigma_{21}$

$$
\sigma_{22}=\sigma_{11}, \sigma_{12}=-\sigma_{11}
$$

The correlation coefficlent $\rho=\sigma_{12} /\left(\sigma_{11} \sigma_{22}\right)^{1 / 2}=-1$ which means $y_{i}$ and $y_{2}$ are completely anticorrelated. Whenever $y_{1}$ increases by an amount $y_{2}$ has to decrease by the same amount.

The second example is a linear conservation law between three observables like the comporents of the relative and the two intrinsic spins of colliding nuclet.

$$
\begin{aligned}
& c_{x}\left(\vec{y}_{1}, \vec{y}_{2}, \vec{y}_{3}\right)=y_{1 x}+y_{2 x}+y_{3 x}=0 \\
& c_{y}\left(\vec{y}_{1}, \vec{y}_{2}, \vec{y}_{3}\right)=y_{1 y}+y_{2 y}+y_{3 y}-J=0
\end{aligned}
$$




$$
\begin{array}{r}
25 / 26 \\
C_{z}\left(\vec{y}_{1}, \vec{y}_{2}, \vec{y}_{3}\right)=y_{1 z}+y_{2 z}+y_{3 z}=0
\end{array}
$$

For each spatial component $(x, y, z)$ we obtain the relations

$$
\begin{array}{lll}
\sigma_{11}+\sigma_{12}+\sigma_{13}=0 & \sigma_{12}=1 / 2\left(\sigma_{33}-\sigma_{11}-\sigma_{22}\right) \\
\sigma_{12}+\sigma_{22}+\sigma_{23}=0 & \text { or } & \sigma_{13}=1 / 2\left(\sigma_{22}-\sigma_{11}-\sigma_{33}\right) \\
\sigma_{13}+\sigma_{23}+\sigma_{33}=0 & & \sigma_{23}=1 / 2\left(\sigma_{11}-\sigma_{22}-\sigma_{33}\right)
\end{array}
$$

For each direction the 6 variances can be reduced to the 3 independent diagonal ones.

The third example is a motion restricted on a circle

$$
c_{a}(y)=y_{1}^{2}+y_{2}^{2}-R^{2}=0
$$

It may serve as an exercise for the reader. 


\section{Master Equation and Fokker-Planck Equation}

Let us now consider the physically important situation in which the transition proability $w_{2}\left(\vec{u}_{2}, t_{2} \mid \vec{u}_{1}, t_{1}\right)$ does not depend on the time $t_{1}$ explicftly, but only on the interval $t_{2}-t_{1}$. It may still depend on time implicity via its dependence on the macroscopic variables. For example, the Brownian parifcle might experience a changing environment as it is moving in space. This situation will be seen in nucleus-nucleus collisions discussed later on. We abbreviate

$$
W\left(\vec{u}_{1} \mid \vec{u}_{2}-\vec{u}_{1}, t_{2}-t_{1}\right) \equiv w_{2}\left(\vec{u}_{2}, t_{2} \mid \vec{u}_{1}, t_{1}\right)
$$

Thus, $W(\vec{u} \mid \Delta \vec{u}, \tau)$ is the probability of finding the Brownian particle having a velocity $\vec{u}+\Delta \vec{u}$ at time $t+\tau$ provided it had the velocity $\vec{u}$ at time $t$. Using the Chapman-Kolmogorov equation $(1.20)$ one can write

$$
\begin{aligned}
& \frac{\partial W(\vec{v} \mid \vec{u}-\vec{v}, t)}{\partial t}=\lim _{\tau \rightarrow 0} \frac{1}{\tau}\{W(\vec{v} \mid \vec{u}-\vec{v}, t+\tau)-W(\vec{v} \mid \vec{u}-\vec{v}, t)\} \\
& =\lim _{\tau+0}\left\{\int g^{3} v^{\prime} \frac{W\left(\vec{v}^{\prime} \mid \vec{u}-\vec{v}^{\prime}, \tau\right)}{\tau} W\left(\vec{v} \mid \vec{v}^{\prime}-\vec{v}, t\right)-\frac{1}{\tau} W(\vec{v} \mid \vec{u}-\vec{v}, t)\right\}
\end{aligned}
$$

If we now make use of the normalization condition (1.17) we obtain

$$
\begin{aligned}
\frac{\partial}{\partial t} W(\vec{v} \mid \vec{u}-\vec{v}, t) & =\lim _{\tau+0}\left\{\int d^{3} v^{\prime} \frac{W\left(\overrightarrow{v^{\prime}} \mid+\vec{u}-\vec{v}^{\prime}, \tau\right)}{\tau} W\left(\vec{v} \mid \vec{v}^{\prime}-\vec{v}, t\right)\right. \\
& \left.-\int d^{3} v^{\prime} \frac{W\left(\vec{u}||^{+}-\vec{u}, \tau\right)}{\tau} W(\vec{v} \mid \vec{u}-\vec{v}, t)\right\}
\end{aligned}
$$


First, one identifies $\frac{1}{\tau} W\left(\vec{v}, \mid \vec{u}-\vec{v}^{\prime}, \tau\right)$ as the transition rate for changing the velocity from $\vec{v} \rightarrow \vec{u}$ :

$$
\dot{W}_{\vec{v}^{\prime} \rightarrow \vec{u}} \equiv \lim _{\tau+0} \frac{1}{\tau} W\left(\vec{v}^{\prime} \mid \vec{u}-\vec{v}^{\prime}, \tau\right)
$$

and analogously

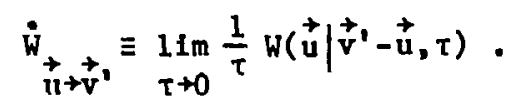

Second, one observes that the distribution function $W_{1}$ can be expressed in terms of $W$ and the initial distributions as

$$
w_{1}(\vec{u}, t)=\int d^{3} v w_{1}(\vec{v}, 0) w(\vec{v} \mid \vec{u}-\vec{v}, t)
$$

If one keeps this in mind and integrates eq. (2.4) with the inftial distribution $W_{1}(\vec{v}, 0)$ one obtains from the Chapman-Kolmogorov incegral equation a balance equation which is called the master equation:

$$
\frac{\partial}{\partial t} w_{1}(\vec{u}, t)=\int d^{3} v \dot{\vec{w}}_{\vec{v} \rightarrow \vec{u}} w_{1}(\vec{v}, t)-\int d^{3} v \dot{\mathrm{w}}_{\vec{u} \rightarrow \vec{v}}^{\vec{v}} \underset{1}{ }(\vec{u}, t)
$$

The first term describes how much probability is gained in a certain velocity bin $\vec{u}$ due to transitions from all other velocity bins $\vec{v}$ into bin $\vec{u}$, whereas the second term is the loss term counting the flux ou'c of bin $\vec{u}$.

In order to derive the Fokker-planck equation we multiply eq. (2.2) by an arbitrary function $g(\vec{u})$ which is smooth and vanished for large values of $\vec{u}$. Then we incegrate over $\vec{u}$ to get 


$$
\int d^{3} u g(\vec{u}) \frac{\partial w(\vec{v} \mid \vec{u}-\vec{v}, t)}{\partial t}=
$$

$$
\begin{aligned}
& \left.\lim _{\tau+0} \frac{1}{\tau} i \int d^{3} v^{\prime} \int d^{3} u W\left(\vec{v}^{\prime} \mid \vec{u}^{+} \vec{v}^{\prime}, \tau\right) W\left(\left.\vec{v}^{+}\right|^{+}-\vec{v}, t\right)\left[\sum_{n=0}^{\infty} \frac{1}{n !}\left(\left(\dot{u}^{+}-\vec{v}^{\prime}\right) \frac{\partial}{\partial v^{+}}\right)^{n} g^{+} \vec{v}^{\prime}\right)\right] \\
& \left.-\int d^{3} u g(\vec{u}) w(\vec{v} \mid \vec{u}-\vec{v}, t)\right\}
\end{aligned}
$$

Here, we made a Taylor expansion of $g(\vec{u})$ around $\vec{v}^{\prime}$. The $n=0$ term cancels the last term because $\int \mathrm{d}^{3} \mathrm{u} W\left(\overrightarrow{v^{\prime}} \mid \overrightarrow{\mathrm{u}}-\vec{v}^{\prime}, \tau\right)=1$ according to eq. $(1,17)$. Hence, we are left with

$\int d^{3} u g(\vec{u}) \frac{\partial W(\vec{v} \mid \vec{u}-\vec{v}, t)}{\partial t}=$

$\left.\sum_{n=1}^{\infty} \frac{1}{n !} \int d^{3} v^{\prime} W\left(\vec{v} \mid \vec{v}^{\prime}-\vec{v}, t\right) \lim _{\tau+0} \frac{1}{\tau} \int d^{3} u w^{\left(\vec{v}^{\prime}\right.} \mid \vec{u}-\vec{v}^{\prime}, \tau\right)\left(\left(\vec{u}-\vec{v}^{\prime}\right) \frac{\partial}{\partial \vec{v}^{\prime}}\right)^{n}\left(\vec{v}^{\prime}\right)$

The averages of the powers of $\left(\vec{u}-\vec{v}^{\prime}\right)$ are called transition moments. It turns out that all transition moments of order 3 and higher are proportional to at least $\tau^{2}$ so that in the $\operatorname{limit} \tau+0$ they vanish:

$$
\begin{aligned}
& \int d^{3} u W\left(\vec{v}^{\prime} \mid \vec{u}^{+}-\vec{v}^{\prime}, \tau\right)\left(u-v^{\prime}\right)_{i}=A_{i}\left(\vec{v}^{\prime}\right) \tau+0\left(\tau^{2}\right) \\
& \int d^{3} u W\left(\vec{v}^{\prime} \mid \vec{u}^{+}-v^{\prime}, \tau\right)\left(u-v^{\prime}\right)_{i}\left(u-v^{\prime}\right)_{k}=B_{i k}\left(\vec{v}^{\prime}\right) \tau+0\left(\tau^{2}\right) \\
& \int d^{3} u W\left(\vec{v}^{\prime} \mid+v^{+}-v^{\prime}, \tau\right)\left(u-v^{\prime}\right)^{n}=0\left(\tau^{2}\right) \quad \text { for } n \geqslant 3
\end{aligned}
$$


30

$\int d^{3} u g(\vec{u}) \frac{\partial W(\vec{v} \mid \vec{u}-\vec{v}, t)}{\partial t}=$

$\int d^{3} v^{\prime} g\left(v^{\prime}\right)\left[-\sum \frac{\partial}{\partial v_{i}^{\prime}}\left(A_{1}\left(\vec{v}^{\prime}\right) w\left(\left.\vec{v}^{+}\right|^{+}-\vec{v}, t\right)\right)+\frac{1}{2} \sum_{1 k} \frac{\partial}{\partial v_{k}^{\prime}} \frac{\partial}{\partial v_{1}^{\prime}}\left(B_{1 k}\left(\vec{v}^{\prime}\right) w\left(\left.\vec{v}^{+}\right|^{+},-\vec{v}, t\right)\right)\right]$

By renaming $\vec{v}$ as $\vec{u}$ and arguing that the integration must hold for all $g(\vec{u})$ we obtain the Fokker-Planck equation

$$
\left.\frac{\partial}{\partial t} W(\vec{v} \mid \vec{u}-\vec{v}, t)=-\sum \frac{\partial}{\partial u_{i}}\left(A_{1}(\vec{u}) W(\vec{v} \mid \vec{u}-\vec{v}, t)\right)+\frac{1}{2} \sum_{1 k} \frac{\partial}{\partial u_{i}} \frac{\partial}{\partial u_{k}}(B)(\vec{u}) W(\vec{v} \mid \vec{u}-\vec{v}, t)\right)(2.14)
$$

The same equation also holds for $w_{1}(\vec{u}, t)$ which can be shown with the help of eq. $(2.6)$. 


\section{A Model for Dissipative Heavy-ion Collisions: The One-body Dissipation Model}

Dissipative phenomena observed in collisions between two atomic nuclef bear great resemblance to a generalized Brownian movement. One of the macroscopic variables is the relative momentum. The corresponding mass is the reduced mass which has to be compared to the mass of the individual nucleons which play the role of the heat bath molecules. The ratio $\mathrm{M} / \mathrm{m}$ is of the order 100 compared to $10^{20}$ in tie Brownian particle case. Therefore, we expect rather violent fluctuations and strong friction forces. On the other hand, heavy-ion collisions are very distinct from a Brownian particle in a heat bath, because one is dealing with a closed system consisting of only a few hundred constituents. Besides this, the initial state has zero temperature, and only during tine interaction time, which is not much longer than the typical time for a direct reaction, the system converts incident kinetic energy into intrinsic excitation and may thus build up a heat bath. Therefore, conventional statistical physics canno, be applied immediately, because there a large external heat bath, coupled to the system of interest, governs the dissipative behavior, whereas in heavy-ion collisions the thermal energy contained in the intrinsic degrees of freedom changes rapioly from zero up to a substantial fraction of the coherent kinetic energy contained in the macroscopic variables.

In the following lectures I shall present a model [Fe84] which describes various aspects of dissipative heavy-ion collisions for center of mass energles ranging from the Coulomb barrier up to several MeV per nucleon above the barrier. The lower limit is imposed by treating classical trajectories, whereas the upper limit is based on the assumption that the mean 
field is well established. This means that the relative velocity of the two nuclel when they touch should be small compared to the Fermi velocity. I shall concentrate on macroscopic properties of large-scale nuclear motion rather than investigating the problem on a completely microscopic level. Only where the microscopic motion affects the macroscopic variables, we have to consider the coupling between the intrinsic and collective degrees of freedom In a microscopic picture. This will be done by evaluating the conditional probability $u(p, q \mid \eta, \tau)$ which will provide the friction and the diffusion tensor. Even then we will not treat nuclear structure effects which could be done and which are certainly present in measured data. The model uses only well-established gross properties of nuclei and concepts of nonequilibrium statistical physics.

Let us denote by $p$ and $q$ a set of $n$ macroscopic variables. Then, the Langevin equation can be written as

$$
\dot{p}_{1}=k_{1}(p(t), q(t))+x_{1}(t) \quad i=1, \ldots, n
$$

Here, $k_{1}(p, q)$ denotes the components of the conservative force which acts between the macroscoplc variables, while $X_{1}(t)$ are the forces originating from the intrinsic degrees of freedom. The mass tensor $M_{1 k}$, which may depend on the coordinates, relates velocities and momenta via

$$
\dot{q}_{1}=\sum_{k} M^{-1}(q(t))_{i k} p_{k} \quad i=1, \ldots, n
$$

Reading eqs. (3.1) and (3.2) term by term tells us that input is required for our model. First of all we have to define the macroscopic variables $\{q\}$. There is no general recipe which tells us how to find them. 
Each dissipative system has to be considered Individually, and physical intuition and experience has to replace stringent reasoning to some extent. Our cholce of variables, is explained in subsection 3.1 . Next we have to define the mass tensor $M(q)$ describing the collective kinetic energy. This will be done in subsection 3.2 . The conservative forces $K_{1}(p, q)$ arc derivatives of a Hamiltonian $H(p, q)$ which contalns a conservative potential $v(q):$

$$
K_{i}(p, q)=-\frac{\partial H}{\partial q_{1}}=-\frac{\partial T}{\partial q_{i}}-\frac{\partial V}{\partial q_{i}}
$$

The kinetic energy is given by

$$
T(p, q)=\frac{1}{2} \sum_{1 k} p_{1} M^{-1}(q)_{1 k} p_{k}
$$

The conservative potential $V(q)$ of the nuclear system will be given in subsection 3.3 . In subsection 3.4 we discuss the dissipation mechanism, which consists of wall and window friction. It is here where we try to understand, on a microsiopic level, the origin of friction in a gas of fermions which have a long mean-free-path (Knudsen gas). The mean value $\frac{1}{\tau}\left\langle\int_{t}^{t+\tau} x_{i}\left(t^{\prime}\right) d t^{\prime}\right\rangle$ of the time averaged fluctuating force will be the friction force, whereas $\frac{1}{\tau}\left\langle\int_{t}^{t+\tau} x_{1}\left(t^{\prime}\right) d t^{\prime} \int_{t}^{t+\tau} x_{j}\left(t^{\prime \prime}\right) d t^{\prime \prime}>\right.$ will describe the diffusion (subsection 3.5 ). In the following sections we have to employ many idealizations and assumptions to simplify the full nuclear many-body problem. The relevant approximations whIch we shall refer to In different places are listed below. They represent a vfew of gross properties of nuclei and nuclear matter [Sw80]. Macroscopic: The number of particlpating nucleons is large, so that Individual particle degrees of freedom need not be considered explicitly. The dynamics of the nuclear system are described by a few collective variables. 
Leptodermous: Nuclei have a thin skin. The width of the diffuse surface is small compared to the size of the nuclear system. This follows from the saturation property of nuclear matter and the short range of the nuclear force.

Low temperature: The excitation energy of the nucleus is such that the corresponding temperature is small compared to the Fermi energy. The nucleons can be treated as filling a nearly degenerate Fermi sea obeying Fermi-nirac statistics.

Long mean free path: Due to the Pauli exclusion principle two-body collisions between nucleons are strongly suppressed in a nucleus at low temperature. The nucleons form a Knudsen gas enclosed in the mean potential Semi-classical: The collective velocities are small compared to the Fermi velocity. This is necessary to obtain equilibration in internal degrees of freedom on a time scale which is smaller than the one for collective variables.

\subsection{Macroscopic variables}

If the relative velocity of the two colliding nuclei is small compared to the Fermi velocity, it is assumed that the nuclear matter of the system does not behave much differently than in ordinary nuclef. The nuclei still form a leptodermous (thin-skinned) system with a well-defined surface and a unform deusity distribution in the interfor independent of the actual shape, the intrinsic excitation energy or the flow velocities. Therefore, we shall characterize the macroscopic appearance of the nuclear system by a family of shapes with sharp surfaces. The volume of the shapes is conserved, so that the uniform mass and charge densities are the same as before the 
collision. Next we restrict the shapes to possess axial symmetry. If we take the z-axis to be the symmetry axis, then the shape is given by the profile function $P(z)$ which generates the configuration by a rotation around the z-axis.

In the following we shall use three degrees of freedom for the shape, denoted by $\{s, \sigma, \Delta\}$. Thus the profile is uniquely given by the function $\mathrm{P}(s, \sigma, \Delta ; z)$. For positive values of $\sigma$ we are using the shapes proposed by Blockl and Swlateck1 [BS81,B180] which are defined as

$$
\begin{array}{ll}
\mathrm{R}_{1}^{2}-\mathrm{z}^{2} & \text { for }-\mathrm{R}_{1}<z<z_{1}, \\
a_{1}+b z+c z^{2} & \text { for } \mathrm{z}_{1}<z<z_{2}, \\
\mathrm{P}^{2}(s, \sigma>0, \Delta ; z)= & \text { for } z_{3}<z<z_{4}, \\
a_{2}+b z+c z^{2} & \text { for } z_{4}<2<s+R_{2} .
\end{array}
$$

The family includes single shapes and bipartite shapes ausisting of two fragments (see fig. 3.1.1). In elther case the two end pieces of a shape are composed of spheres with radil $R_{1}$ and $R_{2}$. At the matching points, $z_{1}$ and $z_{4}$, 

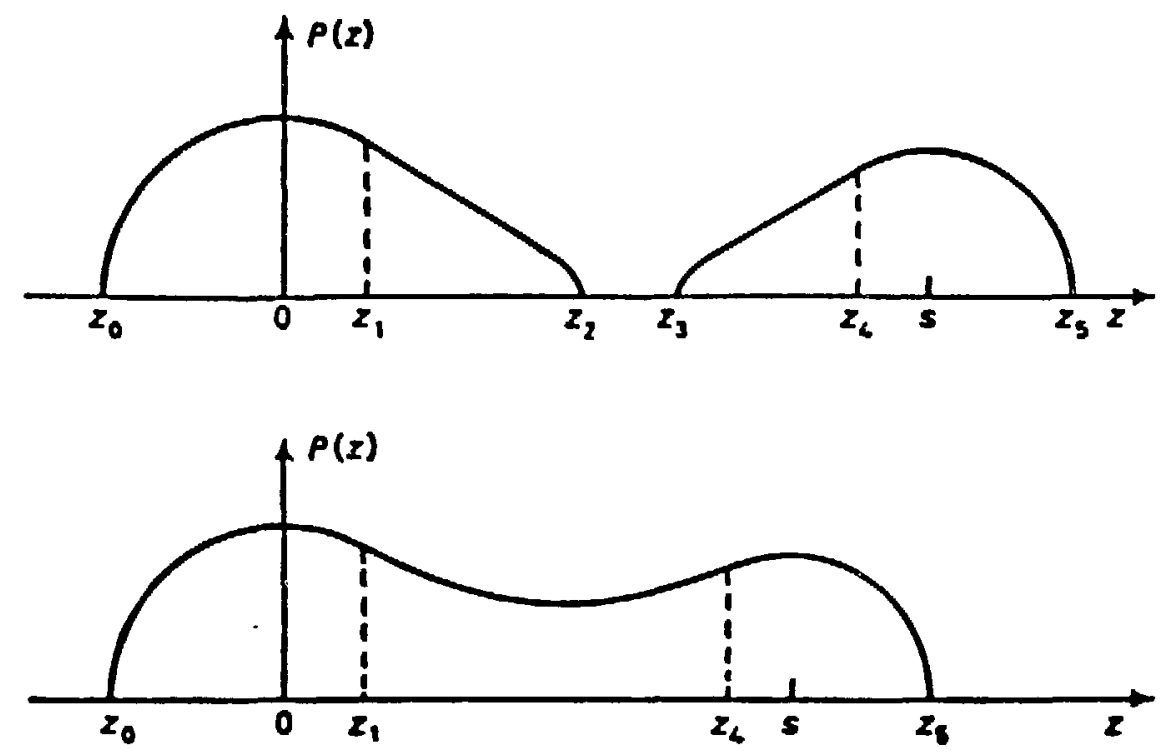

Fig. 3.1.1 Profile of shapes as a function of $z$. Rotation around the $z$-axis generates axially symmetric separated (bipartite) and single nuc lear objects.

they are smoothly connected (continuous first derivative with respect to $z$ ) to a hyperboloid or a spheroid. For the single shape the parameters $a_{1}$ and $a_{2}$ are identical while $z_{2}$ and $z_{3}$ are obsolete. For the bipartite shape $a_{1}$ and $a_{2}$ are chosen such that the volume in each fragment is conserved separately for fixed mass asymmetry; then $z_{2}$ and $z_{3}$ are the positions of the inner tips of the two fragments. All of the parameters $R_{1}, R_{2}, a_{1}, a_{2}, b, c, z_{1}, z_{2}, z_{3}, z_{4}$ depend on the three collective degrees of freedom $s, \sigma, \Delta$, where

$s=$ distance between two sphere centers,

$$
\sigma=\frac{v_{0}-(4 \pi / 3) R_{1}^{3}-(4 \pi / 3) R_{2}^{3}}{v_{0}}=\frac{\text { neck volume }}{\text { total volume }}
$$




$$
\Delta=\frac{R_{1}-R_{2}}{R_{1}+R_{2}}=\text { asymmetry }
$$

Here, $V_{0}$ is the total volume of the shape and is independent of $\{s, \sigma, \Delta\}$, 8 characterizes the elongation, $\sigma$ is a measure of the neck and of the deformation and $\Delta$ detern.ines the asymmetry. Only for bipartite shapes is $\Delta$ equivalent to the mass asymmetry. For thin necks this is still the case, whereas for compact shapes with fat necks $\Delta$ becomes merely the shape asymmetry and mass asymmetry loses its meaning. We shall call the subset of shapes which contains bipartite shapes and shapes where the neck radius is smaller than $R_{1}$ and $R_{2}$ the di-nuclear regime; we will speak about the mono-nuclear regime if the shapes have no necks anymore. Mono-nuclear shapes are, for example, the ones at $(s, \sigma)=(6,0),(11, .3)$ displayed in fig. 3.1 .2 .

For the above-defined shapes we encounter a boundary at $\sigma=0$ for bipartite shapes (two spheres) and a boundary at negative o for shapes where the neck part has disappeared $\left(z_{1}=z_{4}\right)$ leaving just two intersecting spheres. Actual trajectory calculations show that the system wants to cross these boundaries and enter into undefined regions. The physical reason for this is that the long-ranged Coulomb force prefers slightly oblate deformations in the approach phase instead of two spheres $(\sigma=0)$. Therefore, we attach to the family of shapes defined in eq. (3.1.1) at $\sigma=0$ another family of shapes which have oblate deformations. For negative values of $\sigma$ the profile function is defined by 

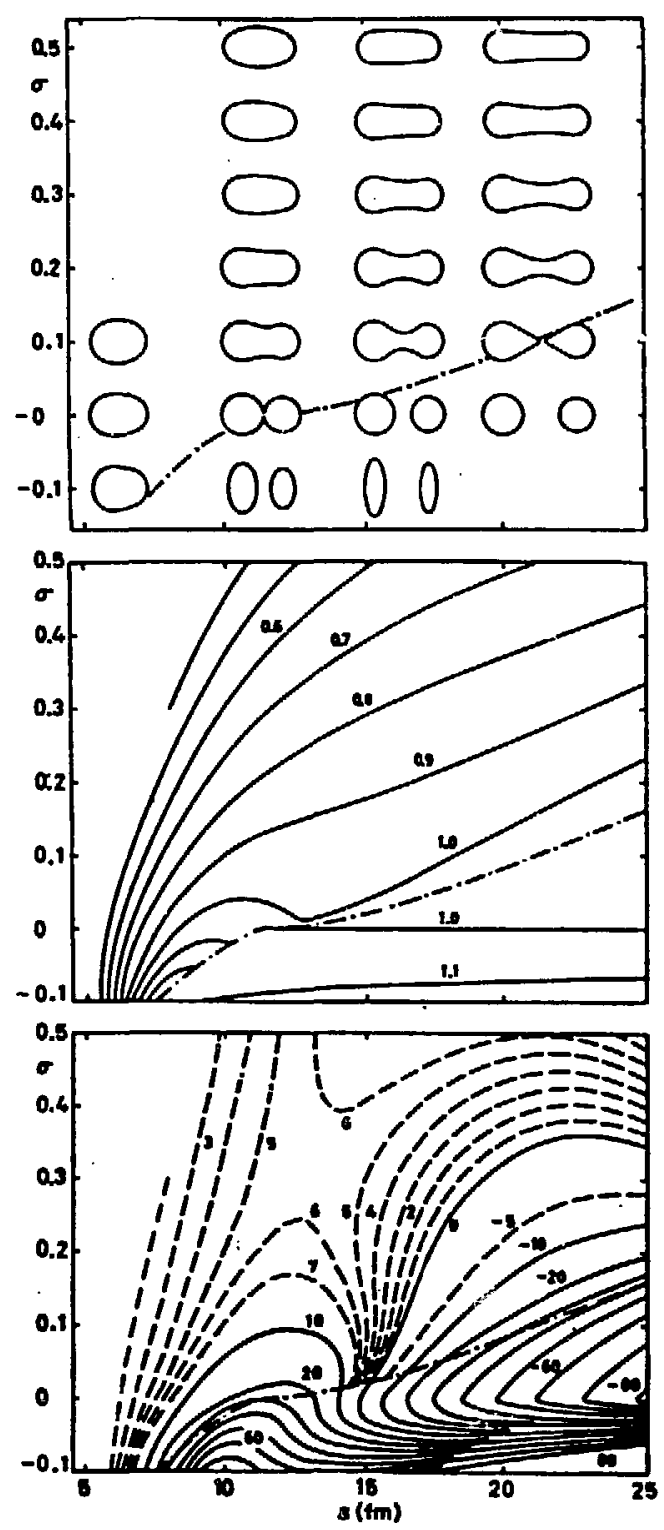

Fig. 3.1.2 Upper part: family of shapes for fixed asymmetry as a function of $s$ and $\sigma, A=225$ and $\Delta=0.08$. Center part; contour plot of the diagonal matrix element $M_{1}$ for the motion in the s-direction divided by the reduced mass $\mu$ in the Werner-Wheeler collective flow tensor, $A=225, \Delta=0.08$. Lower part: contour plot of the potential energy calculated with the folding potential, $A=225, Z=93, \Delta=0.08$. Energles are in $\mathrm{MeV}$ and with respect to the compound sphere. 


$$
\mathrm{P}^{2}(\mathrm{~s}, \sigma<0, \Delta ; z)=\alpha \mathrm{P}^{2}(\tilde{\mathrm{s}}, 0, \Delta ; \tilde{z})
$$

with $\tilde{s}=\alpha s$ and $\tilde{z}=\alpha z$.

The deformation parameter $\alpha$ depends on $s$ and $\sigma$ and is chosen to make the transition from negative to positive $\sigma$ as smooth as possible, where

$$
\alpha=1-2\left(\frac{\varepsilon}{R_{0}}\right)^{2} \sigma
$$

with $R_{0}$ the radius of the compound sphere $\left((4 \pi / 3) R_{0}^{3}=v_{0}\right)$. Thus we extend the shapes into negative values of $\sigma$ by taking a shape at $\sigma=0$ and squeezing it in the $z$-direction while blowing it up radially. Transformation (3.1.3) conserves volume and the distance $s$ between the outer ellipsoids remains unchanged. For bipartite shapes we have just two ellipsoids at a distance s with a ratio of the semi-axes given by $\alpha^{j / 2}$. The reason for not taking $\alpha$ as a fourth degree of freedom which would bring us to the shapes used by Nix and co-workers [Ni69,MN76] will become clear when we define the rotational degrees of freedom. For head-on collisions or induced fission a could be an Independent variable since the end pieces do not rotate.

The above-defined family of shapes is plotted as a function of $s$ and $\sigma$ for fixed asymmetry $\Delta=0.08$ in fig. 3.1.2. Included in the graph is the scission line which divides the areas of single and bipartite shapes. Moving from large to small values of $s$ we go over from stretched or separated shapes to very compact shapes resembling more a single deformer. nucleus. Positive growing $\sigma$ denotes increasing necks and negative $\sigma$ oblate shapes.

In addition to the three shape degrees of freedom there are three rotational degrees of freedom. The axially symmetric shapes defined above may be rotated around an axis perpendicular to the symmetry axis by an angle $\theta$ 
(see fig. 3.1.3). In addition, the sphere on the outer ends may be rotated Independently by the angles $\theta_{1}$ and $\theta_{2}$. Thus, we are able to describe the transfer of relative to intrinsic angular momentum of two colliding nuclei without further assumptions about sticking, etc. However, the Independent rotations about $\theta_{1}$ and $\theta_{2}$ Imply that we ought to have spheres at the outer tips of the shape. If we rotate ellipsoids, we cannot keep the axial symmetry. This is the case for negative $\sigma$. The computed trajectorles show that the oblate deformations never become really large in the entrance channel, so that we can live with the compromise between treating the rotation properly and not having an unphysical boundary. Altogether, we are dealing with six collective degrees of freedom denoted by

$$
q=\left\{q_{1}, q_{2}, \ldots, q_{6}\right\}=\left\{s, \sigma, \Delta, \theta, \theta, \theta_{2}\right\} .
$$

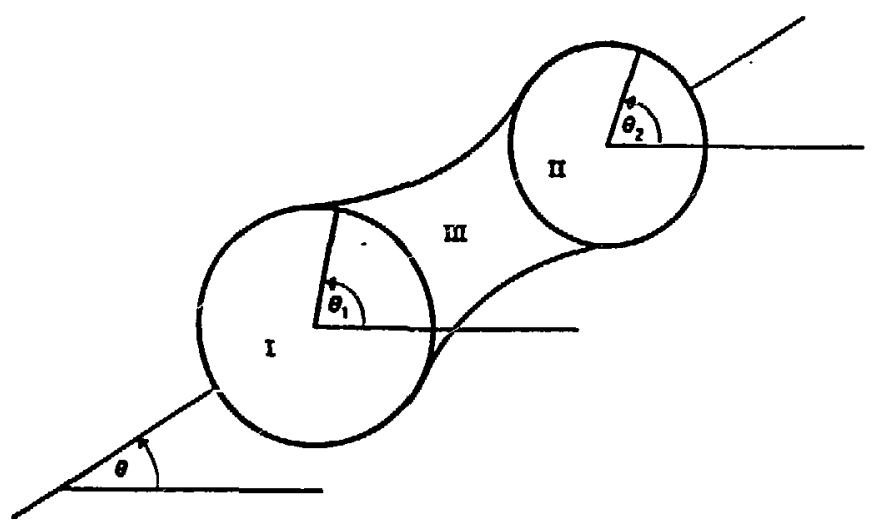

Fig. 3.1.3 Rotational degrees of freedom 


\subsection{Kinetic energy}

The collective kinetic energy of a gas or fluld is given by

$$
T=\frac{1}{2} \int \rho(\vec{r})[\vec{v}(\vec{r})]^{2} d^{3} r=\frac{\rho_{m}}{2} \int_{\text {shape }}[\vec{v}(\vec{r})]^{2} d^{3} r
$$

where $\rho(r)$ is the matter density and $\vec{v}(\vec{r})$ the collective velocity at position $\vec{r}$. In our case the matter density is uniformly distributed within the shape and is zero outside. The velocity fleld $\vec{v}(\vec{r})$ has to be chosen in accordance with the collective degrees of freedom.

The time-dependent changes of the shape result in a collective flow $\vec{u}(\vec{r})$ which rearranges the matter to maintain a uniform density distribution. The boundary condition being that the velocity component normal to the surface is equal to the normal component of the surface velocity $\vec{w}$ :

$$
\overrightarrow{\mathrm{un}}=\overrightarrow{\mathrm{wn}} \text { at the surface. }
$$

Here $\vec{n}$ is the unit vector normal to the surface and $\overrightarrow{w n}$ is given by

$$
\underset{\mathrm{wn}}{+\overrightarrow{\mathrm{N}}}=\frac{\mathrm{dP}}{\mathrm{dt}}\left[1+\left(\frac{\partial \mathrm{P}}{\partial \mathrm{z}}\right)^{2}\right\}^{-1 / 2}
$$


Figure 3.2.I explains this relation. The task is to find an adequate velocity field. The assumption of incompressible Irrotational flow together with the boundary condition (3.2.2) weuld uniquely define the whole velocity fleld $\vec{u}(\vec{r})$ and thereby the kinetic energy for the shape degrees of freedom. However, the solution of the resulting Laplace equation is numerically too costly.

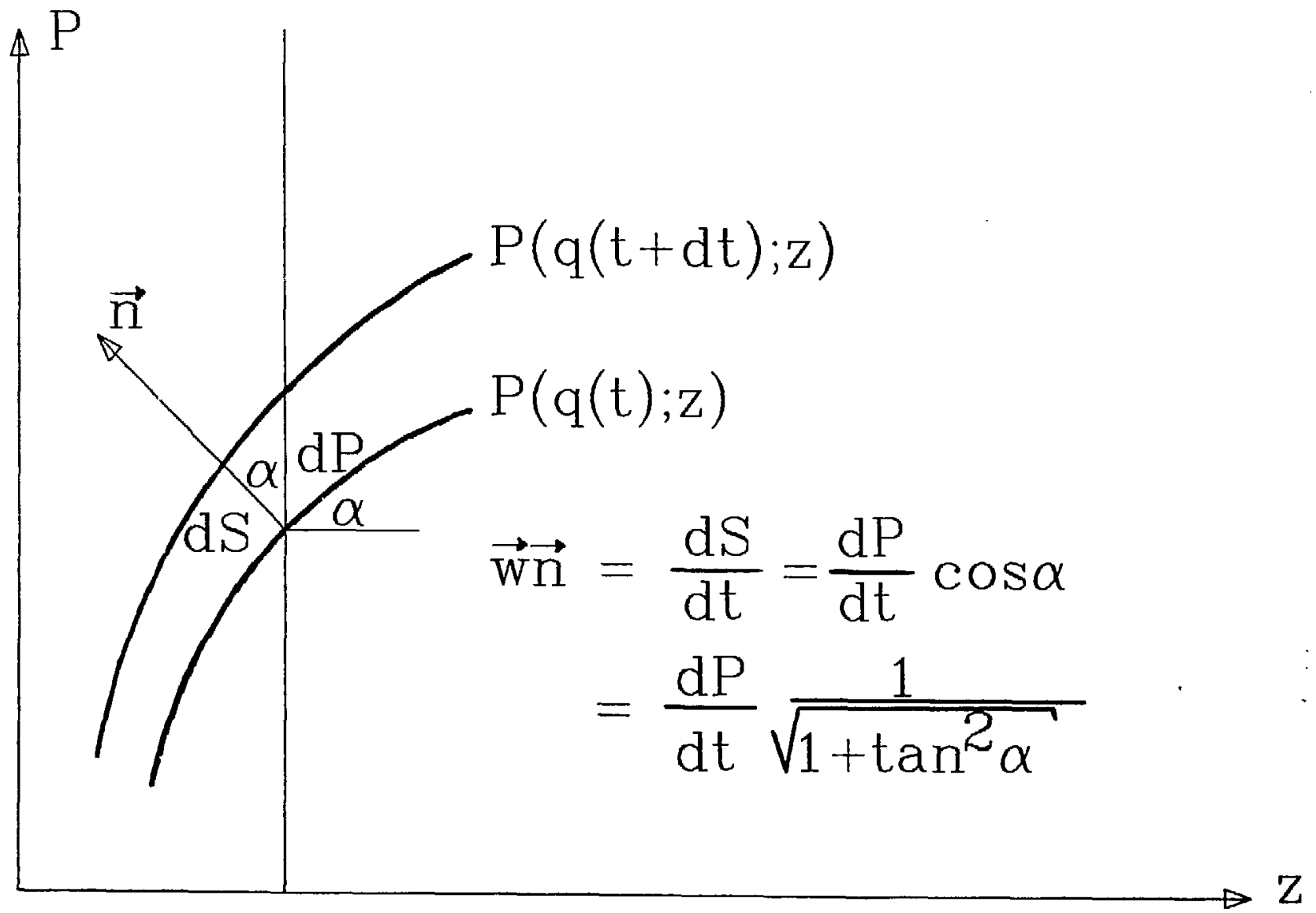

Fig. 3.2.1 Derivation of eq. (3.2.3.) 
Therefore, we are using the Werner-Wheeler flow [Ke64] which is an approximation to the irrotational flow [DS76].

The first assumption for the Werner-Wheeler flow is Incompressibility.

$$
\operatorname{div} \vec{u}=0
$$

For axial symmetry the velocity field has a radial component $u_{r}$ and a zcomponent $\mathbf{u}_{2}:$

$$
\operatorname{div} \vec{u}=\frac{1}{r} \frac{\partial}{\partial I}\left(r u_{I}\right)+\frac{\partial u_{z}}{\partial z}=0
$$

The second assumption is that the flow is such that a plane surface at zposition $\zeta$ remains plain and is moving so that the volume on each side remains unchanged :

$$
\frac{d}{d t} \int_{z_{0}}^{\zeta} \pi P(q ; z)^{2} d z=\frac{d}{d t} \int_{\zeta}^{z_{5}} \pi P(q ; z)^{2} d z=0
$$

The profile $P(q ; z)$ of the shape has been defined in eqs. $(3.1 .1)-(3.1 .3)$ as a function of the collective parameter $q$ and the spatial coordinate $z$ (c.f. fig. $3.2 .2 j$. 


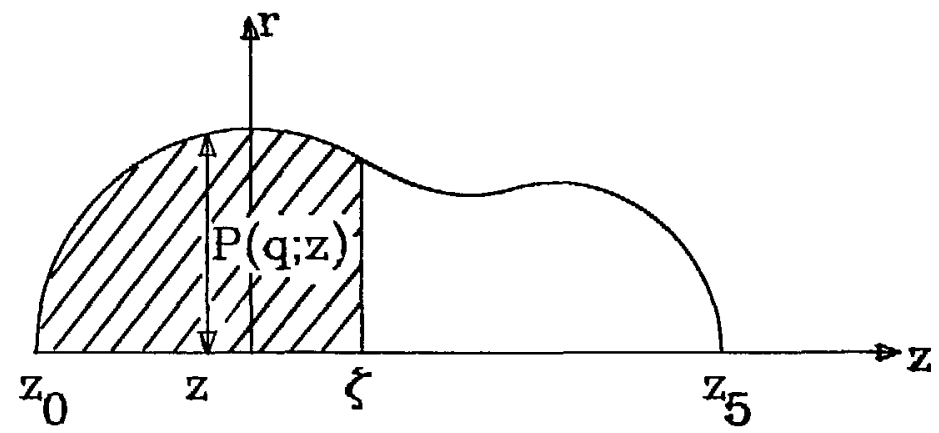

Fig. 3.2.2 Profile of the shape of the nuclear aystem as a function of the collective parameters $q$ and the spatial coordinate $z$

Using eq. (3.2.6) we determine the z-component $u_{z}$ of the velocity

field

$$
\begin{aligned}
& 0=\frac{d \zeta}{d t} P(q ; \zeta)^{2}-\frac{d z}{d t} P(q ; z)^{2}+\int_{z_{0}}^{\zeta} \frac{d}{d t}\left(P\left(z^{\prime} ; q\right)^{2}\right) d^{\prime} \\
& \frac{d \zeta}{d t}=-\frac{1}{P(q ; z)^{2}} \int_{z_{0}}^{z} \frac{d}{d t}\left(P\left(q ; z^{\prime}\right)^{2} d z^{\prime}\right.
\end{aligned}
$$

Since we want to count all velocities with respect to the center of mass velocity, $u_{z}$ is given by

$$
u_{z}=-\frac{1}{P(q ; z)^{2}} \int_{z_{0}}^{z} \frac{d}{d t}\left(P\left(q ; z^{\prime}\right)^{2} d z^{\prime}-\frac{d z c m}{d t} \quad \text { for } z_{0}<z<z z_{2}\right.
$$

or equivalently

$$
u_{z}=-\frac{1}{P(q ; z)^{2}} \int_{z_{5}}^{z} \frac{d}{d t}\left(P\left(q ; z^{\prime}\right)^{2}\right) d z^{\prime}-\frac{d z c m}{d t} \quad \text { for } z_{3}<z<z_{5} \quad(3.2 .10)
$$


where $z_{0}$ is the position of the left-hand tip and $z_{5}$ that of the right-hand t1p where $P\left(q ; z_{0}\right)=P\left(q ; z_{5}\right)=0$. Equation $(3.2 .10)$ follows from the seconot part of eq. $(3.2 .6)$.

Equations (3.2.9) and (3.2.10) are valld equally for bipartite and single shapes. In the case of the latter the range of validity for (3.2.9) reaches up to $z_{5}$ and for $(3.2 .10)$ down to $z_{0}$. The position of the center of mass 18 denoted by $\mathrm{cm}$.

Since $u_{z}$ depends only on $z$ the incompressibility condition (3.2.5) can be integrated to get the radial velocity by incorporating the boundary condition $(3.2 .2)$ to yield

$$
u_{r}=-\frac{r}{2} \frac{\partial u_{z}}{\partial z} \text {. }
$$

Since the time derivative in eqs. $(3.2 .9)$ and $(3.2 .10)$ enters via the collective velocities $\dot{q}_{i}$ as

$$
\frac{d}{d t}=\sum_{i=1}^{3} \dot{q}_{i} \frac{\partial}{\partial q_{1}},
$$

the Werner-Wheeler flow velocity $\stackrel{\vec{u}}{\text {, where }}$

$$
\vec{u}=u_{\mathbf{r}} \vec{e}_{\mathbf{r}}+u_{z} \overrightarrow{\dot{\epsilon}}_{z} .
$$

is determined completely by the shape parameters and their time derivatives. 
The velocities of the rotational degrees of freedom give rise to an additional flow. The total collective velocity field in the left-hand sphere, therefore, is given by

$$
\vec{v}(\vec{r})=\stackrel{+}{u}(\vec{r})+\vec{\omega}_{1} \times \vec{r}-\stackrel{t}{w} \times \vec{r}_{\mathrm{cm}} \text { for } \stackrel{t}{\mathrm{r}} \varepsilon \mathrm{I},
$$

in the right-hand sphere by

$$
\vec{v}(\vec{r})=\stackrel{+}{u}(\vec{r})+\stackrel{+}{w}_{2} \times\left(\stackrel{+}{r}-\vec{s}^{+}\right)-\vec{w}_{w} \times\left(\vec{s}-\vec{r}_{\mathrm{cm}}\right) \text { for } \overrightarrow{\mathrm{r}} \in I I
$$

and in the neck region by

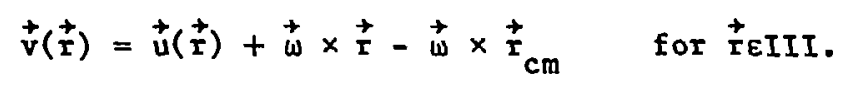

The areas I, II and III are Indicated in fig. 3.1.3. The a..gular velocities $\vec{w}, \vec{w}_{1}$ and $\vec{w}_{2}$ are given by

$$
\vec{\omega}=\dot{\theta}_{\mathrm{e}}, \vec{\omega}_{1}=\dot{\theta}_{1} \overrightarrow{\mathrm{e}}_{\mathrm{y}}, \vec{\omega}_{2}=\dot{\theta}_{2} \overrightarrow{\mathrm{e}}_{\mathrm{y}},
$$

Inserting the collective velocity field $\vec{v}(\vec{r})$ into eq. $(3.2 .1)$ results in the kinetic energy $T$ which is expressed in the following equation (3.2.18). It 1s worthwhile to note that cross-terms between $\vec{u}$ and the rotational velocities due to the axial symmetry do not contribute in eq. $(3.2 .1)$

$$
T=\frac{1}{2} \quad \sum_{1, j=1}^{6} \dot{M}_{1 j} \dot{q}_{1} \dot{q}_{j}
$$


If we use eqs. $(3.2 .9)-(3.2 .12)$, the mass tensor $M_{i j}(1, j=1,2,3)$ for the shape degrees of freedom is as follows:

$$
M_{i j}=p_{m} \pi \int\left\{\frac{1}{2} B_{i} B_{j}+P^{2} A_{i} A_{j}\right\} d z, i, j=1,2,3,
$$

with

and

$$
\begin{aligned}
& \tilde{A}_{1}=-\frac{1}{p^{2}} \int_{z_{0}, z_{5}}^{z} \frac{\partial p^{2}}{\partial q_{1}} d z^{\prime}, \\
& A_{i}=\tilde{A}_{i}-\frac{\partial z_{c m}}{\partial q_{1}}, \\
& \frac{\partial z_{c m}}{\partial q_{i}}=\frac{\pi}{v_{0}} \int_{z_{0}}^{z_{5}} \frac{\partial p^{2}}{\partial q_{1}} z d z
\end{aligned}
$$

$$
B_{i}=\frac{1}{2} \frac{\partial p^{2}}{\partial z} \tilde{A}_{1}+\frac{1}{2} \frac{\partial p^{2}}{\partial q_{1}} \text {. }
$$

As an illustration we display in the center part of fig. 3.1 .2 the matrix element $M_{11}$ of the Werner-Wheeler flow mass tensor divided by the reduced mass. For bipartite shapes it is very close to unity and becomes smallet for compast sharas.

The mass tensor $M_{1 j}(i, j=4,5,6)$ for the rotational degrees of freedom is diagonal. The total moment of inertia is

with

$$
I_{\text {tot }}=\rho_{m} \pi \int_{z_{0}}^{z_{5}}\left\{\frac{1}{4} p^{4}+p^{2} z^{2}\right\} d z-p_{m} v_{0} z_{c m}^{2}
$$

$$
z_{c m}=\frac{\pi}{v_{0}} \int_{z_{0}}^{z_{5}} \mathrm{p}^{2} z d z
$$


The relative moment of inertia $\mathrm{M}_{44}$ is the difference between the total and the two moments of inertia $I_{1,7}$ of the spheres (ellipsoids for $\sigma<0$ ):

$$
\begin{aligned}
& M_{44} \equiv I_{\text {rel }}=I_{\text {tot }}-I_{1}-I_{2}, \\
& M_{44,55} \equiv I_{1,2}=\rho_{m} \frac{4 \pi}{15} R_{1,2}^{5}\left[\alpha+\frac{1}{\alpha^{2}}\right] .
\end{aligned}
$$

For $\sigma>0$ we have $\alpha=1$ and, for $\sigma<0$, $\alpha$ measures the oblate deformation (see eq. (3.1.3). Matrix elementte that cross-terms between $\star \star \star \star \star \star$ and the rotational velocities due to the axial symmetry do not contribute in eq. $(3.2 .1)$

Although the rotational velocity fields are defined properly only when the two spheres are not intersecting (fig. 3.1.3), we use the abovedefined mass tensor for all shapes. Once the nuclei have come so close that the two spheres overlap appreciably, the system has reached most likely the sticking limit, so that only $I_{\text {tot }}$ enters the equations of motion and $I_{1}$ and $I_{2}$ become obsolete.

\subsection{Potential energy}

We want to associate with each shape an energy of the nuclear system. A unified nuclear potential for large-scale collective motion has been developed by Krappe, Nix, Sierk and Möller [KN79,MN81]. They propose a double volume integral of a Yukawa-plus-exponential folding function for the nuclear part, $v_{n}$, of the potential energy:

$$
v_{n}=-\frac{c_{s}}{8 \pi^{2} r_{0}^{2} a^{3}} \int_{\text {shape }} d^{3} r d^{3} r^{\prime}\left\{\frac{1}{a}-\frac{2}{\left|\vec{r}-\vec{r}^{\prime}\right|}\right\} \exp \left[-\frac{\left|\vec{r}-\vec{r}^{\prime}\right|}{a}\right] \text {. }
$$


The parameters have been fitted to ground-state energies and fission barrier heights. We are using the set given in [KN79], where

$$
\begin{aligned}
& c_{s}=a_{s}\left(1-k_{s}\left(\frac{N-Z}{A}\right)^{2}\right) \\
& a_{s}=21.7 \mathrm{MeV} \\
& \kappa_{s}=3 \\
& r_{0}=1.18 \mathrm{fm} \\
& a=.65 \mathrm{fm}
\end{aligned}
$$

The volume term of [KN79] does not show up here, because the total volume is kept fixed and therefore it would add only a constant to the energy.

For a single sphere configuration eq. (3.3.1) can be integrated and the following explicit expression has been obtained

$$
V_{n}(\text { sphere })=\left[1-3\left(\frac{a}{R_{0}}\right)^{2}+\left(\frac{R_{0}}{a}+1\right)\left[2+3 \frac{a}{R_{0}}+3\left(\frac{a}{R_{0}}\right)^{2}\right] e^{-2 R_{0} / a}\right] c_{s} A^{2 / 3}
$$

The leading term for nuclei with radil $R_{0}>$ a is the surface energy $c_{s} A^{2 / 3}$. The Yukawa-plus-exponential potential may be regarded as a replacement of the liquid drop surface energy. Ft -thermore, it includes the nuclear attraction between two nuclei, which $\rightarrow$ imrortant for separated shapes and necked-in shapes, where two nuclear surfaces are within the surface diffuseness or are within the range of the nuclear interaction. The nuclear interaction between two spherical nuclei before they touch is compared with other nuclear potentials in fig. 3.3.1 (taken from [KN79]). 


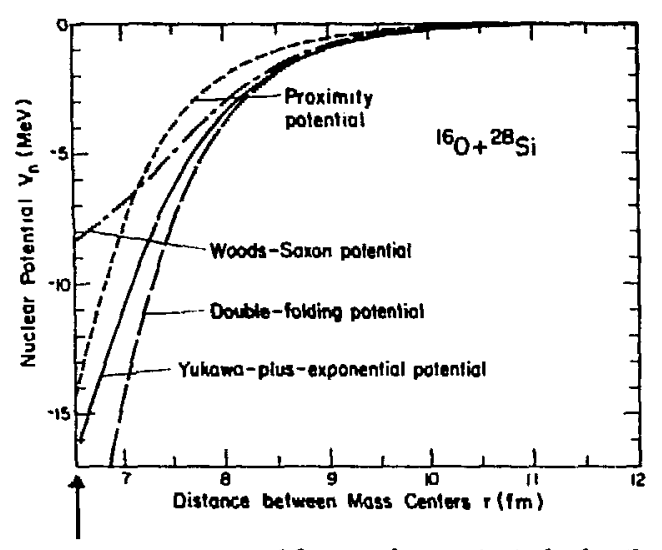

Comparison of four nuclear potentials for the ${ }^{16} \mathrm{O}+{ }^{25}$ si system. The proximity potential is obtained from Ref. 32, the double-folding potential is obtalned from Ref. 26, and the Woods-Saxon potential is obtained from Rev. 54 .

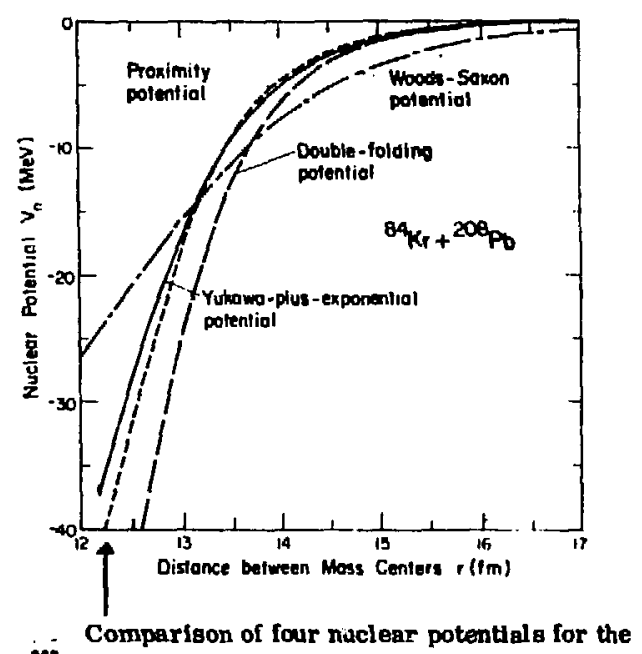

${ }^{4} \mathrm{Kr}+{ }^{200} \mathrm{~Pb}$ system. The proximity potentlal is obtalned from Ref. 32, the double-folding potential is obtained from Ref, 26, and the Woods-Saxon polential is obtained from Ref. 55.

Fig. 3.3.1. Nuclear potentials. The arrows indicate the distance at which the two spheres touch. Reproduced with the permission of the American Physical Society and the author.

The global part of the interaction is the long-ranged Coulomb force, $V_{c}$, which is calculated assuming a uniform charge distribution, $\rho_{c}$, with a sharp surface.

$$
v_{c}=\frac{\rho_{c}^{2}}{2} \int_{\text {shape }} d^{3} r d^{3} r^{\prime} \frac{1}{\left|\vec{r}-\overrightarrow{r^{\prime}}\right|}
$$

Although the finite surface thickness 1s, to a certain extent, incorporated Into the Yukawa-plus-exponentlal folding potential, the Coulomb energy is calculated for a sharp surface only. 
The double-folding integrals in eqs. (3.3.1) and (3.3.4) a re calculated numerically. In order to reduce numerical effort the sixfold integral has been transformed to a threefold integral by using a twofold application of the Gauss divergence theorem and the axial symmetry of the shape [KN79]. The resulting integrals are

$$
\begin{aligned}
V_{n, c}=E_{n, c} \int d z d z^{\prime} d \phi f_{n, c}(r) & P(z)\left[P(z)-P\left(z^{\prime}\right) \cos \phi-\frac{d P(z)}{d z}\left(z-z^{\prime}\right)\right] \\
& P\left(z^{\prime}\right)\left[P\left(z^{\prime}\right)-P(z) \cos \phi-\frac{d P\left(z^{\prime}\right)}{d z^{\prime}}\left(z^{\prime}-z\right)\right]
\end{aligned}
$$

where

$$
r^{2}=P(x)^{2}+P\left(z^{\prime}\right)^{2}-2 P(z) P\left(z^{\prime}\right) \cos \phi+\left(z-z^{\prime}\right)^{2}
$$

with

$$
E_{n}=\frac{c_{s}}{4 \pi r_{0}^{2}} \text { and } f_{n}(r)=\frac{1}{r^{4}}\left\{2-\left[\left(\frac{r}{a}\right)^{2}+2 \frac{r}{a}+2\right] e^{-r / a}\right\}
$$

for the nuclear part, and

$$
E_{c}=\frac{\pi}{6} \rho_{c}^{2} \text { and } f_{c}(r)=\frac{1}{r}
$$

for the Coulomb part. The energles depend on the three shape degrees of freedom $(s, \sigma, \Delta)$ via the dependence of the profile function $P(q ; z)$. The q-dependence was not written down explicitly in eqs. (3.3.5) and (3.3.6). The total energy is the sum of the nuclear plus the Coulomb part 


$$
v(s, \sigma, \Delta)=v_{n}+v_{c}
$$

In fig. 3.1 .2 we displayed a contour plot of the potential energy $v(s, \sigma, \Delta)$ for flxed asymmetry $\Delta=.08$ and a total mass of $A=225$ and charge of $z=93$. For smal1 $\sigma$ and large $s$ we see the binary valley through which in a heavy-ion collision the nuclel enter into the landscape. Close to the touching point of two spheres ( $s=11.3 \mathrm{fm}, \sigma=0$ ) there is the Coulomb barrier which has to be passed in order to bring the two nuclei in close contact. At ( $s=10.1 \mathrm{fm}, \infty=0.25)$ a saddle point divides the two regions where the system can gain energy either by fusing or by separating into two fragments.

\subsection{One-body dissipation}

The one-body dissipation picture makes use of the long mean free path of a nucleon in a nucleus at not too high an excitation energy. The reason for this is that the accessible phase space to final states of two-body collisions is blocked to a large extent by the pauli principle. The other aspect is the mean field of the nuclear object acting as a confining vessel. Altogether the nucleons are assumed to behave like a Knudsen gas, 1.e. they move almost freely inside the shape and then bounce elastically at the wall. The picture is very similar to the shell model and to the time-dependent Hartree-Fock model.

Dissipation occurs when particles which are about to hit the wall do not possess a mean velocity equal to the wall velocity. Thus, after scattering with the wall, their velocity distribution shows a mismatch with the rest of the gas. Now enters a randomization hypothesis which states that the bounced particles obtain the velocity distribution of the bulk before they h1t the wall again [BB78, Gr75]. 
The window friction mechanism is of similar nature. In this case we consider two gases with different mean velocities in contact at a window. Again, particles crossing the window show a mismatch in their velocity distributions with respect to the gas they are entering. The randomization assumption makes sure that the particles have forgotten their original velocity distribution before they return to the window.

To get the friction force $F_{1}$, we have to derive the rate of dissipated energy $\dot{Q}$ for both wall and window friction:

$$
F_{1}=-\frac{1}{2} \frac{\partial \dot{q}}{\partial \dot{q}_{1}}=-\sum_{j} R_{1 j} \dot{q}_{j} .
$$

where $R_{1 j}$ is the friction tensor. Since $\dot{Q}$ will be a bilinear form of the velocities, we can define a friction tensor $R_{1 j}$ as done in eq. (3.4.1) which depends on $q_{1}$ only.

In deriving the friction coefficients for wall and window dissipation we need some knowledge about the time evolution of the intrinsic degrees of freedom. Since the nucleons in the nucleus have a long mean free path, let us study the evolution of a Ferml gas where two-body collisions are absent. The most convenient and Intultive way is to consider the one-body Wigner function (the definition of the wigner function in terms of the statistical operators is given in Appendix A where we also discuss its properties in some detall). Not only the expectation values of one-body operators are formally identical tu the classical expressions but also the time evolution under the influence of a mean fleld is quite similar. In this case the Pauli exclusion principle is taken care of by the Llouville theorem and need not be considered separately. The Lisuville theorem states that in the absence of two-body collisions the phase space distribution behaves like 
an Incompressible fluid. A certain phase space cell can only change $i$ ts shape but always keeps its volume. Thus, a cell of size $(2 \pi h)^{3}$ containing a fermion will always have the space to accommodate this fermion irrespective of how it gets moved around and disturbed by the mean field. If it is going to enter an area of phase space that is being occupied by another particle, the time evolution is such that the occupied space is being emptled as the other phase space cell is moving in.

Let us consider the time evolution of the Wigner function for a onedimensional system. In fig. 3.4 .1 we display the time evolution for a gas which does not feel any forces. Each point moves with a certain velocity $v$ along a straight line in the $x$ direction. The upper two little boxes show how

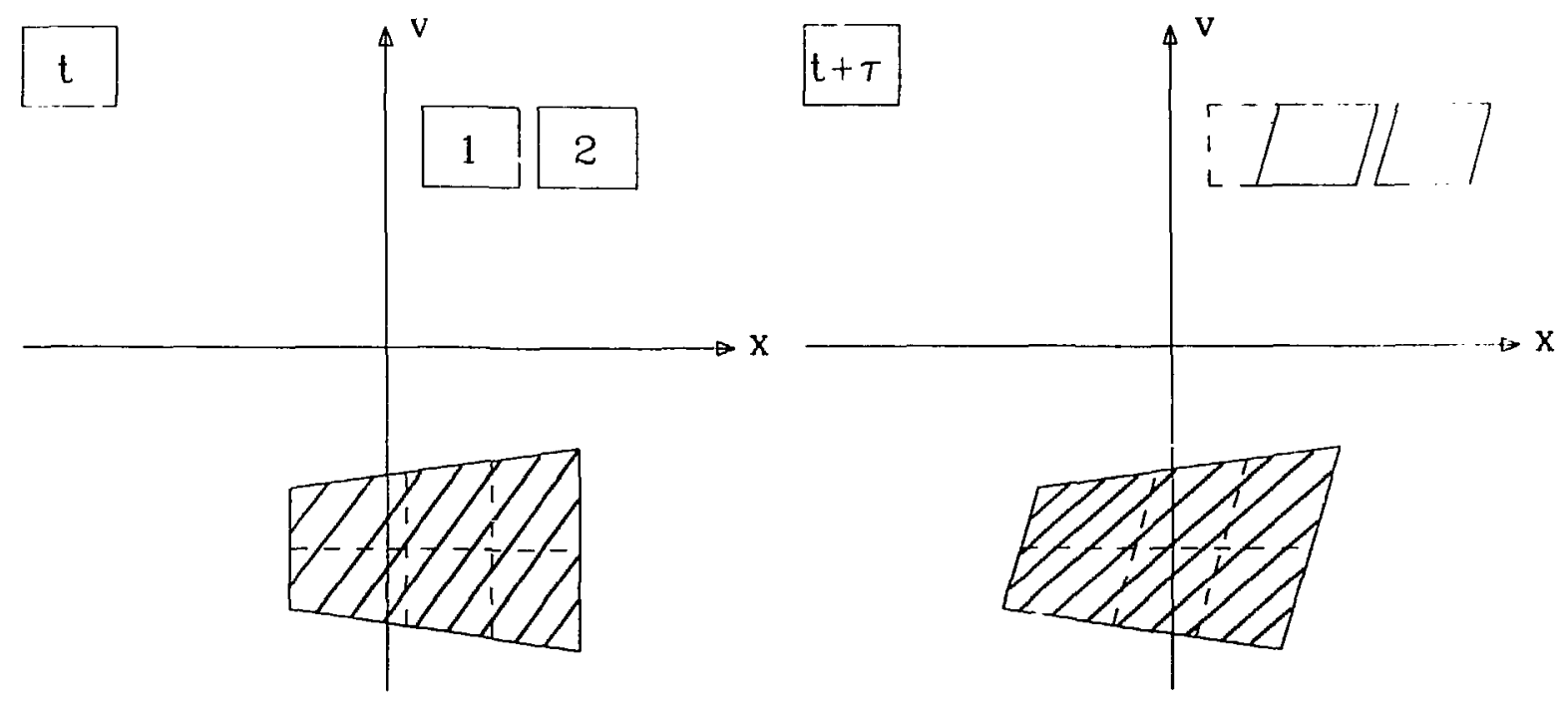

Fig. 3.4.1 Time evoluison of the Wigner function $f^{w}(v, x, t)$ in the absence of any forces. Each point in phase space moves during a time $\tau$ with a velocity $v$ by an amount $v t$ in coordinate space. 
the Pauli exclusion principle is satisfied. The particle sitting in 1 on the left hand side is going to move into the space of 2 irrespective if this is filled or empty because the conceivable particle in the cell originally located at 2 is going to move away. In the lower half we would like to illustrate what indistinguishability meaus. Here, we have a larger portion of phase space which may contain several, say six, fermions. One would now make a division into six celils of size $(2 \pi h)$ as indicated. These six cells would move and distort as a function of time. Or in another language: we are following the evolution of six time-dependent single particle wave functions which have a momentum and coordinate distribution as indicated by the phasespace cells. The total wave function would be a slater determinant made out of these six single particle states. Similar to invariance of a slater determinant under a unitary transformation among the occupied single particle states, the Wigner function is independent of the way in which it is divided up into phase space cells, one could also imagine a time-independent regular grid representing a time-independent basis. Then the motion of a particle would mean occupying and emptying of states. Another Interesting aspect is the movement of a hole which is identical to the movement of a particle.

If there is a potential the motion behaves accordingly. An example of a linear step in the potential is given in fig. 3.4.2. This example, in which a finite step in the mean field keeps part of the Fermi gas bounded to the left and lets only the fast particles escape to the right, might illustrate what happens if the mean velocity of the Fermi gas with respect to the velocity of the mean field is high enough to allow for particle emission, as in the promptly emitted particle (PEP) picture. 

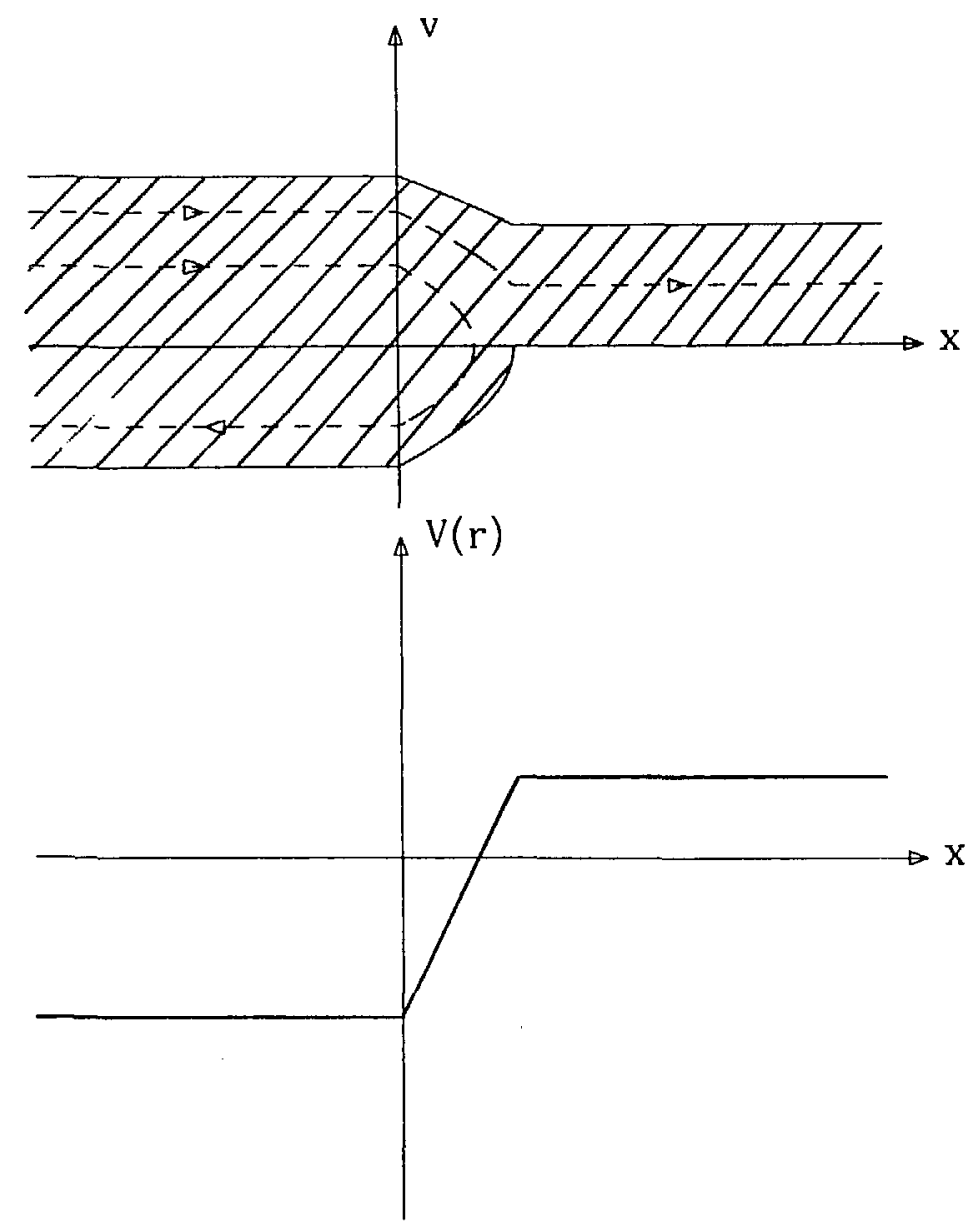

Fig. 3.4.2 A Fermi gas which is partially held back by a finite step in the mean field (lower part). Particles which are fast enough escape to the right while slowing down. Dashed lines indicate trajectories of phase space cells.

of course, one should keep in mind that in a small system one cannot expect sharp edged distributions; the finite penetration probability into the classically forbidden areas of the potential, also smears out the edges. Nevertheless, this simplified view provides valuable insight without solving the time-dependent single particle Schrödinger equation. 


\subsubsection{Wall dissipation}

First let us consider the wall dissipation for a one-dimensional Knudsen gas. In fig. 3.4 .3 we show a schematis of the one-body phase-space density for a gas close to a moving wall at time $t\left(1, h_{,}, s^{)}\right.$and a time t+r (r.h.s.upper and lower). The distribution is that of a Fermi gas with drift velocity $v_{d}$ and Fermi velocity $v_{F}$. In the upper graph on the r.h.s. the wall is moving with the velocity $w$ which is less than $v_{d}$. After time $\tau$ the wall has moved by $w \tau$. The fastest particles close to the
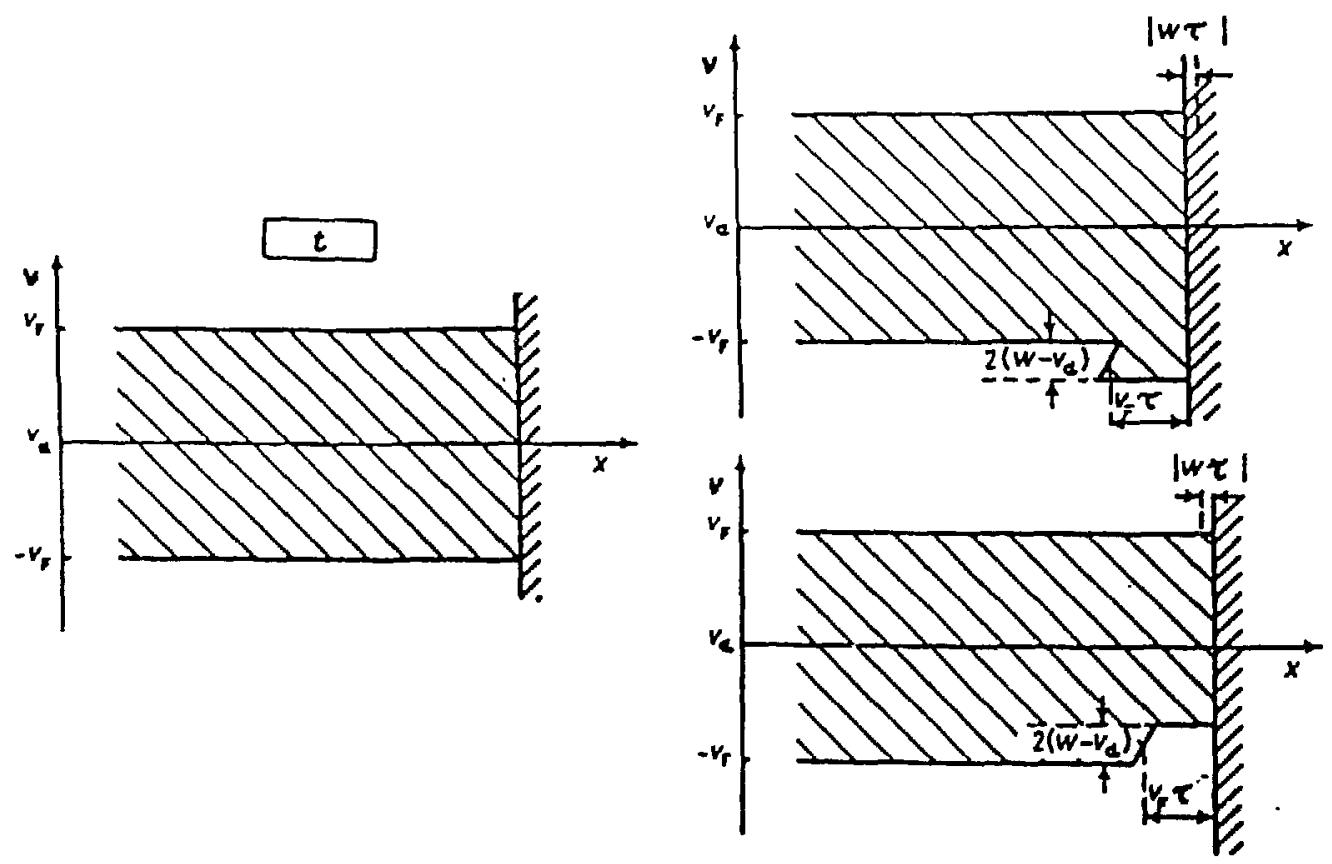

$t+\tau$ $w<v_{c}$

Fig. 3.4.3 Illustration of the wall dissipation in the one-dimensional oneparticle phase space. The hatched area indicates the occupied part. 
wall which have a velocity of $v_{d}+v_{F}$ have bounced and appear now at the velocity $2 w-v_{d}-v_{F}$. The other particles after colliding with the wall reappear according to their velocities. The distribution of $w<v_{d}$ shows a corner of mismatched particles which tend to slow down the drift velocity as they equilibrate with the bulk. This mechanism continues until $v_{d}=w$. The lower graph on the r.h.s. is for $w>v_{d}$ and shows a lack of particles in the lower right-hand corner (holes). This time the holes will increase $v_{d}$ until it reaches $w$.

We are now interested in the difference $\Delta p$ of the total momentum of the gas at time $t+\tau$ between the case in which the wall velocity $w$ differs from the drift velocity $v_{d}$ and that in which they are equal:

$$
\frac{\Delta \mathrm{p}}{\tau}=\frac{1}{\tau} \int \mathrm{w}_{1}(\mathrm{v}) \mathrm{mv} \mathrm{dv}
$$

$W_{1}(v) d v$ is the number of particles with velocity $v$ which have bounced off the wall during the time $\tau$ and did not end up in previously occupied phase space:

$$
w_{1}(v)=f_{0}|(v-w) \tau| g_{b}(2 w-v)\left(1-g_{b}(v)\right),
$$

where $f_{0}$ is the phase-space density and $g_{b}(v)$ is the velocity distribution of the particles before they hit the wall:

$$
g_{b}(v)=g_{r}\left(v-v_{d}\right)
$$

where $g_{F}(v)$ denotes the Fermi distribution $\left(0<g_{F}<1\right)$. Using the simple geometry of fig. 3.4 .3 and eq. $(3.4 .2), \Delta p$ can be calculated: 


$$
\Delta p=f_{0} 2\left(w-v_{d}\right) v_{F}{ }^{T} v_{F}
$$

Here we assumed $w-v_{d}<<v_{F}$. The result is just the number of particles in the little plece sticking out the lower right-hand corner $\left.\left(f_{0}{ }^{2\left(w-v_{d}\right.}\right) v_{F} \Delta t\right)$ times the momentum relative to the bulk $\left(m v_{F}\right)$. Furthermore, using $f_{0} m=F_{i l i} / 2 v_{F}$, we get

$$
\frac{\Delta p}{\tau}=\rho_{m} v_{F}\left(w-v_{d}\right)
$$

where the mass density is denoted by $\rho_{m}$. If this momentum is dissipated as supposed in the randomization hypothesis, then the corresponding energy loss per unit time is given by

$$
\dot{Q}_{1}=\frac{\Delta p}{\tau}\left(w-v_{d}\right)=\rho_{m} v_{F}\left(w-v_{d}\right)^{2}
$$

At this point we should like to comment on the effect of Pauli blocking in wall friction. As discussed in section 3.4 , one would expect no contribution from particles which bounce off the wall into occupied phasespace cells (c.f. fig. 3.4.3) because it is only a rearrangement among occupied cells. However, we excluded this from the very beginning (c.f. eq. $(3.4 .3)$ ) and counted only particles in the little piece sticking out where the phase space has been empty previously. In the derivation by Swiatecki et al. [BB78] for a classical gas all particles which hit the wall during the time $\tau$ ase included. This results in a pressure term plus the dissipative term given in eq. $(3.4 .2)$ or $(3.4 .6)$. It is this pressure term which could be called Paulf forbidden. However, this term is not entering the wall formula. Thus 
Pauli blocking does not decrease the standard wall friction! By the way, the finite pressure of a Fermi gas at zero temperature exists and is not Pauli forbidden.

After this discussion it is a stralghtforward exercise to get the expression for the rate of dissipated energy in the three-dimensional case:

$$
\dot{Q}_{1}=\rho_{m} \bar{v} \int_{\text {surface }} \mathrm{df}\left(\overrightarrow{\mathrm{n}}\left(\overrightarrow{\mathrm{w}}-\vec{v}_{\mathrm{d}}\right)\right)^{2}
$$

where it is assumed that $\left|\vec{w}-\vec{v}_{d}\right| \ll<v_{F}$. The surface area element df has $\vec{n}$ as its normal unit vector and moves with velocity $t_{\text {, while }} t_{d}$ denotes the drift velocity of the gas particles which are about to hit the surface element df. (c.f. fig. 3.2.1). The integration has to be performed over the surface of the shapes defined in section 3.1 .

If we assume that the drift velocity has only a z-component

$$
\stackrel{\vec{v}}{\mathrm{~d}}_{\mathrm{d}}=\mathrm{v}_{\mathrm{d}} \overrightarrow{\mathrm{e}}_{\mathrm{z}}=\sum_{\mathrm{i}} \Phi_{1} \dot{\mathrm{q}}_{\mathbf{1}} \dot{\mathrm{e}}_{\mathrm{z}}
$$

and use eqs. $(3.2 .3),(3.2 .12),(3.4 .1)$ and $(3.4 .9)$, the wall dissipation tensor is given by

$$
\begin{aligned}
& R_{i j}^{(1)}=\rho_{m} \bar{v} \frac{\pi}{2} \cdot \\
& { }_{z_{5}}^{z_{j}} \frac{\left(\partial \mathrm{p}^{2} / \partial q_{i}+\left(\partial \mathrm{P}^{2} / \partial z\right) \dot{\phi}_{1}\right)\left(\partial \mathrm{P} / \partial q_{1}+\left(\partial \mathrm{P}^{2} / \partial z\right) \phi_{j}\right)}{\left\{\mathrm{P}^{2}+\left(\frac{1}{2}\left(\partial \mathrm{P}^{2} / \partial z\right)\right)^{2}\right\}^{1 / 2}} d z
\end{aligned}
$$

and the energy dissipation rate is 


$$
\dot{Q}^{\text {wall }}=\sum_{i, j=1}^{3} R_{i j}^{\text {wall } \dot{q}_{i} \dot{q}_{j}}
$$

The choice of the drift velocity, $\stackrel{\mathbf{v}}{d}_{\mathrm{d}}$ will be discussed later. It should not be mixed up with the collective velocity $\vec{u}$ used in section 3.2 to obtain the kinetic energy. Close to the wall $\vec{u}$ is a mean between those particles which have just bounced and those which are about to bounce, therefore $\vec{n}(\vec{w}-\vec{u})=0$, whereas $\vec{v}_{d}$ is the mean velocity of only those particles which are flying towards the wall, and $\vec{n}\left(\vec{w}-\vec{v}_{d}\right)$ need not be zero. In a knudsen gas two velocity distributions may coexist at the same position.

The choice of the drift velocity fleld will be discussed in section $\because .4 .3$ where we combine the wall and the window friction. 
3.4.2 Exchange of particles, momentum and entropy

In this section we derive in a unified way transport coefficients

for the particle flux, momentum flux and entropy flux through the window

between two nuclei. We shall calculate microscopically the transport

coefficients for the window friction, the heat conduction, the resistance

against mass flow and cross terms which correspond to the Peltier effect

observed at the junction of two metals. The window friction coefficlent will

be derived a second time in section 3.5 together with the ciffusion

coefficients. There we use the same ideas as in the previous section. One talks of a window because two touching nuclei produce a mean single particle

field which resembles two containers with an opening between them. Through

this window particles will be exchanged and equilibration will take place.

The basic idea of the model is to regard each nucleus as a Fermi gas described by a statistical operator. A moving Fermi zas is completely spectfied by the following three extensive quantities:

$$
\begin{array}{ll}
\text { excitation energy } & \mathrm{E}^{*}=\langle\underset{\sim}{\mathrm{H}}\rangle-\mathrm{E}_{\mathrm{gS}} \\
\text { number of particles } & \mathrm{N}=\langle\underset{\sim}{N}\rangle \\
\text { center of mass momentum } & \stackrel{+}{\mathrm{P}}=\langle\stackrel{\dot{\sim}}{\sim}\rangle
\end{array}
$$

Hence, the statistical operator which contains only this information is of the form

$$
\underset{\sim}{R}=1 / Z \exp \{-\beta \underset{\sim}{H}-\alpha \underset{\sim}{N}-\underset{\sim}{\stackrel{P}{P}}\}
$$

The Lagrange parameters are composed of three intensive quantities:

$$
\begin{array}{ll}
\text { tempera cure } & T=1 / \beta \\
\text { chemical potential } & u=-\alpha / \beta+\frac{1}{2} \overrightarrow{m u}^{2} \\
\text { center of mass velocity. } & \vec{u}=-\vec{\gamma} / \beta
\end{array}
$$

Here, m denotes the mass of one nucleon. However, since there is no a priori equilibration between the two nuclei, one considers two temperatures $\mathrm{T}_{1,2}$, twr 
chemical potentials $\mu_{1,2}$, and two c.m. velocities $\vec{u}_{1,2}$ (c.f. Fig. 3.4.4). If we make the Idealization that the nucleus can be described by an Ideal Fermi gas it is sufficient to consider only the velocity distribution of the particles. This can be deduced from the statistical operator as

$$
\begin{aligned}
& g\left(\stackrel{\vec{v}}{,} \alpha_{i}, \beta_{1}, \vec{\gamma}_{1}\right)=\left\{1+\exp \left(\beta_{1} \frac{1}{2} \mathrm{mv}^{+2}+\vec{\gamma}_{1} \mathrm{mv}+\alpha_{1}\right)\right\}^{-1} \\
& =\left\{1+\exp \left(\left[\frac{1}{2} m\left(\vec{v}-\vec{u}_{1}\right)^{2}-\mu_{1}\right] / T_{1}\right)\right\}^{-1}
\end{aligned}
$$

Here, the index $1=1,2$ refers to nucleus 1 and 2 , respectively. Therew 1 th, the Intensive quantities depend on position in space in such a way that they are uniform in each nucleus but show a finite discontinuity at the "window" between the two nuclei. The particle exchange between the two gases will tend to equilibrate the mismatch in temperatures, chemical potentials and velocities by meins of a flux of entropy, particles and momentum between the gases.

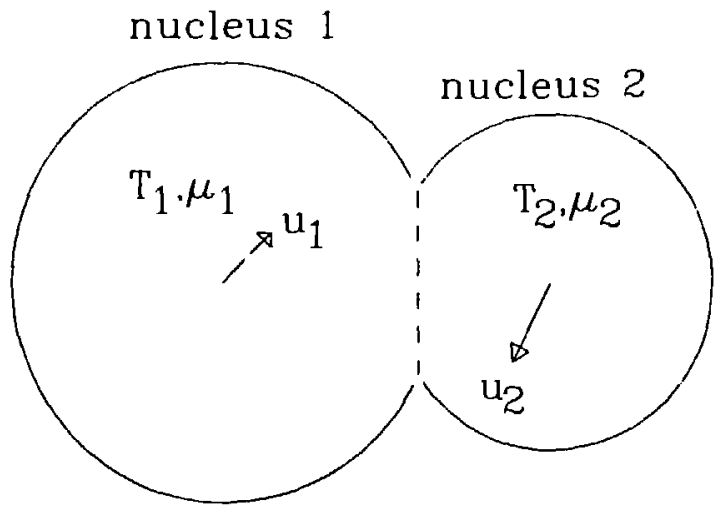

Fig. 3.4.4 The two nuclet are regarded as moving Fermi gases which are specified by their temperatures, chemical potentials and velocities. There exists communication between the two gases by means of particle exchange through the window.

The flux of $\ln (z)$ through a hole in the container enclosing the gas, which is given by 


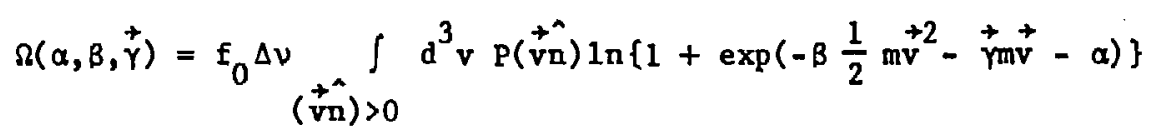

is a quantity from which all other desired fluxes can be calculated. In eq. (3.4.15), $f_{0}$ is the phase space density, $\Delta v$ is tive a rea of a window element and $\hat{n} \mathbf{i t s}$ normal vector. If there is a potential barrier in the window then the particles have a certain probability, P, to cross the barrier. The limit $(\vec{v} \hat{n})>0$ for the integration means that all velocities are represented in a frame in which the window has zero velocity. The particle flux out of nucleus 1 Into nucleus 2 is the negative of the first derivative with respect to $\alpha$ :

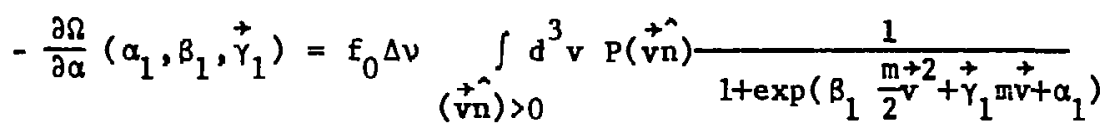

Similarly the momentum and entropy currents can be written as:

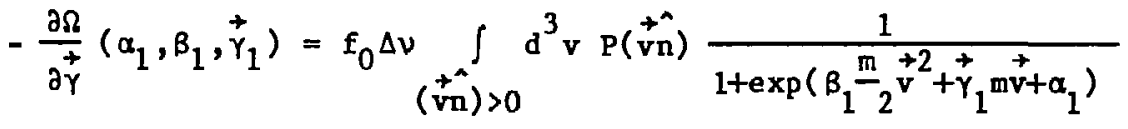

$$
\begin{aligned}
& \dot{s}_{1}=\left[\Omega-\alpha \frac{\partial \Omega}{\partial \alpha}-\beta \frac{\partial \Omega}{\partial \beta}-\dot{\gamma} \frac{\partial \Omega}{\partial \dot{\gamma}}\right]\left(\alpha_{1}, \beta_{1}, \vec{\gamma}_{1}\right)
\end{aligned}
$$

It is easy to verify that the corresponding fluxes from nucleus 2 into nucleus 1 are given by:

$$
\begin{aligned}
& -\frac{\partial \Omega}{\partial \alpha}\left(\alpha_{2}, \beta_{2},-\vec{\gamma}_{2}\right) \\
& +\frac{\partial \Omega}{\partial t}\left(\alpha_{2}, \beta_{2},-\vec{\gamma}_{2}\right) \\
& {\left[\Omega-\alpha \frac{\partial \Omega}{\partial \alpha}-\beta \frac{\partial \Omega}{\partial \beta}-\stackrel{+}{\frac{\partial}{\gamma}}\right]\left(\alpha_{2}, \beta_{2},-\vec{\gamma}_{2}\right)}
\end{aligned}
$$


The net fluxes are then the mutual differences. For example, the net particle flux which 18 also the rate of change of the particle number in nucleus one can be expressed as:

$$
\dot{A}_{1}=\frac{\partial \Omega}{\partial \alpha}\left(\alpha_{1}, \beta_{1}, \vec{\gamma}_{1}\right)-\frac{\partial \Omega}{\partial \alpha}\left(\alpha_{2}, \beta_{2},-\vec{\gamma}_{2}\right)
$$

If the difference in the intensive quantities are small one can use a Taylor expansion around the mean values in order to linearize eq. $(3.4 .20)$

$$
\begin{aligned}
\dot{A}_{1} & =\frac{\partial \Omega}{\partial \alpha}\left(\alpha_{0}, \beta_{0}, 0\right)+\left.\frac{\partial^{2} \Omega}{\partial \alpha^{2}}\right|_{0}\left(\alpha_{1}-\alpha_{0}\right)+\left.\frac{\partial^{2} \Omega}{\partial \alpha \partial \beta}\right|_{0}\left(\beta_{1}-\beta_{0}\right)+\left.\frac{\partial^{2} \Omega}{\partial \alpha \partial+\vec{\gamma}}\right|_{0}\left(\vec{\gamma}_{1}-\vec{\gamma}_{0}\right) \\
& -\frac{\partial \Omega}{\partial \alpha}\left(\alpha_{0}, \beta_{0}, 0\right)-\left.\frac{\partial^{2} \Omega}{\partial \alpha^{2}}\right|_{0}\left(\alpha_{2}-\alpha_{0}\right)-\left.\frac{\partial^{2} \Omega}{\partial \alpha \partial \beta}\right|_{0}\left(\beta_{2}-\beta_{0}\right)-\left.\frac{\partial^{2} \Omega}{\partial \alpha \partial \dot{\gamma}}\right|_{0}\left(-\vec{\gamma}_{2}-\vec{\gamma}_{0}\right)
\end{aligned}
$$

The symbol $\left.\right|_{0}$ means that all the derivatives have to be taken at the mean values:

with

$$
\alpha_{0}=-\frac{\varepsilon_{F}}{T_{0}}, \beta_{0}=\frac{1}{T_{0}}, \vec{\gamma}_{0}=0
$$

$$
\varepsilon_{F}=\frac{1}{2}\left(\varepsilon_{F 1}+\varepsilon_{F 2}\right), T_{0}=\frac{1}{2}\left(T_{1}+T_{2}\right), \dot{\gamma}_{0}=\frac{1}{2}\left(\dot{\gamma}_{1}+\dot{\gamma}_{2}\right)
$$

The choice $\vec{\gamma}_{0}=0$ corresponds to the situation where the window moves with a velocity $\frac{1}{2}\left(\vec{u}_{1}+\vec{u}_{2}\right)=0$. The differences in the intensive quantities are defined as

$$
\Delta \mathrm{T}=\mathrm{T}_{2}-\mathrm{T}_{1}, \Delta \mu=\mu_{2}-\mu_{1}, \Delta \overrightarrow{\mathrm{u}}=\overrightarrow{\mathrm{u}}_{2}-\overrightarrow{\mathrm{u}}_{1}
$$


Collecting all terms in eq. $(3.4 .21)$ and using the relations in eq. (3.4.13) to transform back to the old coordinates we get

$$
\dot{\mathrm{A}}_{1}=\left.\frac{\partial^{2} \Omega}{\partial \alpha^{2}}\right|_{0} \frac{1}{\mathrm{~T}_{0}} \Delta \mu+\left[\left.\frac{\partial^{2} \Omega}{\partial \alpha \partial \beta}\right|_{0}-\left.\varepsilon_{\mathrm{F}} \frac{\partial^{2} \Omega}{\partial \alpha^{2}}\right|_{0}\right] \frac{1}{\mathrm{~T}_{0}{ }^{2}} \Delta \mathrm{T}
$$

Here, we have retained only the terms which are of lowest order in $\Delta \mu, \Delta T$ and $\Delta \vec{u}$.

For the change of the momentum of nucleus 1 we get

$$
\dot{\mathrm{p}}_{11}=\frac{\partial \Omega}{\partial \gamma_{1}}\left(\alpha_{1}, \beta_{1}, \vec{\gamma}_{1}\right)+\frac{\partial \Omega}{\partial \gamma_{1}}\left(\alpha_{2}, \beta_{2},-\vec{\gamma}_{2}\right)
$$

The linearization yields

$$
\begin{aligned}
\dot{P}_{1 i} & =\left.2 \frac{\partial \Omega}{\partial \gamma_{i}}\right|_{0}+\left.\underset{k}{\sum} \frac{\partial^{2} \Omega}{\partial \gamma_{1} \partial \gamma_{k}}\right|_{0}\left(\gamma_{1 k}-\gamma_{2 k}-2 \gamma_{0 k}\right) \\
& =\left.2 \frac{\partial \Omega}{\partial \gamma_{i}}\right|_{0}+\left.\underset{k}{\sum} \frac{\partial^{2} \Omega}{\partial \gamma_{i} \partial \gamma_{k}}\right|_{0} \frac{1}{T_{0}} \Delta \vec{u}
\end{aligned}
$$

Terms in $\Delta \mu$ and $\Delta T$ do not appear in lowest order since on the r.h.s. of eq. (3.4.25) there is a plus sign unlike the minus sign in eq. $(3.4 .20)$. For the same reason the first derivative term survives [first term r.h.s. eq. (3.4.26)]. This is just the pressure which is present even in equilibrium $(\Delta \vec{u}=0)$. It does not give rise to a friction force and is balanced by the nuclear interaction. It does not give an additional repulsive force which drives the nuclei apart. If this were so any nucleus in its ground state would explode because one could imagine a fictitious cross section through the nucleus and calculate by the method mentioned above the force which is executed on the two halves. The result would be the cross 
section times the zero-point pressure which is present in any Fermi gas. This force certainly does not cause the nucleus to break because it is balanced by the nuclear interactions which hold the nucleus together. See also [BB78] and the corresponding discussion in section 3.4.1. Therefore, the pressure term is dropped and the friction force resulting from the exchange of momentum is given by

$$
\mathrm{F}_{1}=-\dot{\mathrm{P}}_{11}=-\left.\sum_{\mathrm{k}} \frac{\partial^{2} \Omega}{\partial Y_{i} \partial Y_{k}}\right|_{0} \frac{1}{\mathrm{~T}_{0}} \Delta \vec{u}
$$

The derivation of the entropy flux proceeds in a similar way by using eq. $(3.4 .18)$.

The results of these linearizations can be summarized as a set of coupled thermodynamic equations:

$$
\begin{aligned}
& \dot{A}_{1}=g_{A A} \Delta \mu+g_{A S} \Delta T \\
& \dot{S}_{21}=g_{S A} \Delta \mu+g_{S S} \Delta T \\
& \dot{F}=-\dot{P}_{1}=-\ddot{g} \Delta \vec{u}
\end{aligned}
$$

The mobility coefficient for particle transfer $\mathrm{g}_{\mathrm{AA}}$ is given by:

$$
g_{A A}=\left.\frac{1}{T_{0}} \frac{\partial^{2} \Omega}{\partial \alpha^{2}}\right|_{0}
$$

The equality between the peltier coefficient $g_{A S}$ and the Seebeck coefficlent $g_{S A}$ reflects the Onsager relation: 


$$
g_{A S}=g_{S A}=\frac{1}{T_{0}^{2}}\left[\left.\frac{\partial^{2} \Omega}{\partial \alpha \partial \beta}\right|_{0}-\left.\varepsilon_{F} \frac{\partial^{2} \Omega}{\partial \alpha^{2}}\right|_{0}\right] ;
$$

the entropy conductivity g $g_{S S}$ is calculated as:

$$
g_{S S}=\frac{1}{T_{0}^{3}}\left[\left.\frac{\partial^{2} \Omega}{\partial \beta^{2}}\right|_{0}-\left.2 \varepsilon_{F} \frac{\partial^{2} \Omega}{\partial \alpha \partial \beta}\right|_{0}+\left.\varepsilon_{F}^{2} \frac{\partial^{2} \Omega}{\partial \beta^{2}}\right|_{0}\right] ;
$$

and finally the momentum transport coefficients for the window friction tensor $\ddot{\mathrm{g}}$ are given by

$$
\ddot{\mathbf{g}}=\left.\frac{1}{T_{0}} \frac{\partial^{2} \Omega}{\partial \underset{\gamma}{t} \partial \vec{\gamma}}\right|_{0}
$$

The various second derivatives of $\Omega$ appearing in eqs. (3.4.29)(3.4.32) can be evaluated for the case of a classical penetrability $P$ which appeared in eq. $(3 \cdot 4 \cdot 15)$. Let us consider the case of semi-infinite nuclear matter on either side of a potential barrier at the window as indicated in fig. 3.4.5)

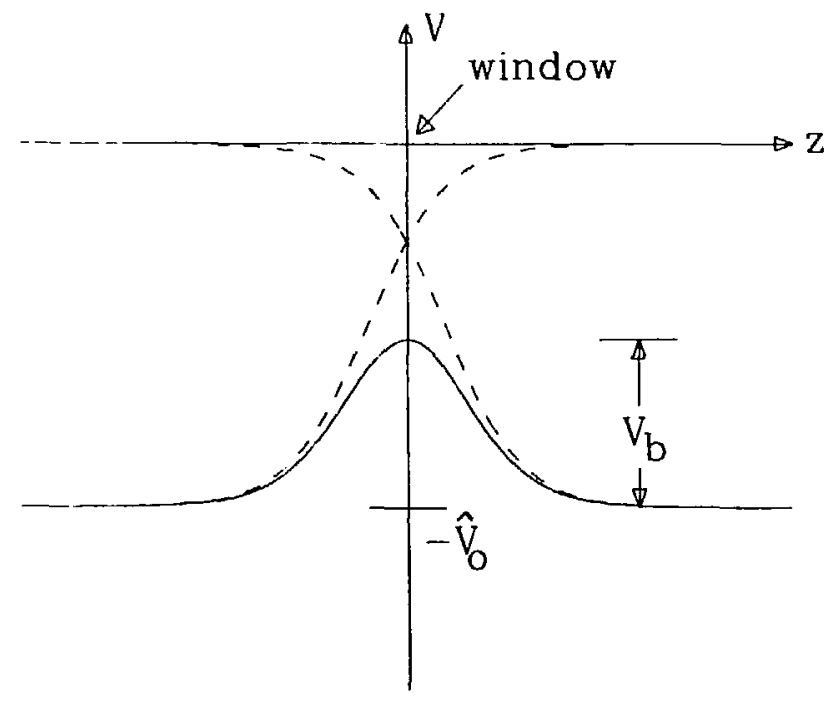

Fig. 3.4.5 Single particle potential in the vicinity of the window. 
The shape of the single particle potential is assumed to be of the form

$$
v_{S}(D, z)=-\frac{\hat{v}_{0}}{1+e^{(z+D / 2) / b}}-\frac{\hat{v}_{0}}{1+e^{-(z-D / 2) / b}}
$$

It depends on the distarice $D$ between the equivalent sharp surfaces which, for now, are assumed to be planes. The helght $v_{b}$ of the barrier seen by a particle is then given by

$$
v_{b}(D)=\hat{v}_{0}\left(1-\frac{2}{1+e^{D / 2 b}}\right) \quad \text { for } D>0
$$

The actual parameters used are $\hat{\mathrm{V}}_{0}=50 \mathrm{MeV}, \mathrm{b}=.85 \mathrm{fm}$; these parameters will reproduce the universal function derfved by Randrup [Ra78] from a Thomas Ferm 1 calculation. The classical penetrability is the following step function

$$
P(\hat{v} \tilde{n})=\theta\left(\frac{m}{2}\left(\vec{v}^{n}\right)^{2}-V_{b}(D)\right)
$$

Using eq. (3.4.35) and $\vec{\gamma}=0$ we can rewrite $\Omega$ (defined in eq. (3.4.15)) in terms of dimensionless functions

$$
\Omega(\alpha, \beta, 0)=\tilde{f}_{0} \frac{1}{\beta^{2}} \int_{-\infty}^{-\beta v_{b}-\alpha} d y \int_{-\infty}^{y} d x \ln \left\{1+e^{x}\right\}
$$

with $\tilde{\mathrm{f}}_{0}=\frac{3}{4} \frac{\rho}{\mathrm{p}_{\mathrm{F}}} \frac{1}{\varepsilon_{F}} \Delta v$, where $\rho, \mathrm{p}_{\mathrm{F}}$ and $\varepsilon_{\mathrm{F}}$ denote the particle density, Fermi momentum and Fermi energy, respectively.

Since the Integral in eq. $(3.4 .36)$ cannot be performed analytically we introduce the abbreviations

$$
L(y)=\ln \left\{1+e^{y}\right\}
$$




$$
\begin{aligned}
& I(y)=\int_{-\infty}^{y} d x L(x) \\
& K(y)=-\int_{\infty}^{y} d x I(x)
\end{aligned}
$$

Using these, we can express the derivatives of $\Omega$ as

$$
\begin{aligned}
& \frac{\partial \Omega}{\partial \alpha}=-\tilde{\mathrm{f}}_{0} \frac{\mathrm{l}}{\beta^{2}} \mathrm{I}\left(-\beta \mathrm{V}_{\mathrm{b}}-\alpha\right) \\
& \frac{\partial \Omega}{\partial \beta}=-\tilde{\mathrm{f}}_{0} \frac{2}{\beta^{3}} K\left(-\beta \mathrm{V}_{\mathrm{b}}-\alpha\right)-\tilde{\mathrm{f}}_{0} \frac{\mathrm{V}_{0}}{\beta^{2}} I\left(-\beta V_{b}-\alpha\right) \\
& \frac{\partial^{2} \Omega}{\partial \alpha^{2}}=\tilde{\mathrm{f}}_{0} \frac{1}{\beta^{2}} \mathrm{~L}\left(-\beta \mathrm{V}_{\mathrm{b}}-\alpha\right) \\
& \frac{\partial^{2} \Omega}{\partial \beta^{2}}=\tilde{\mathrm{f}}_{0} \frac{6}{\beta^{4}} K\left(-\beta V_{b}-\alpha\right)+\tilde{\mathrm{f}}_{0} \frac{4 V_{b}}{\beta^{3}} I\left(-\beta V_{b}-\alpha\right)+\tilde{\mathrm{f}}_{0} \frac{V_{0}}{\beta^{2}} L\left(-\beta V_{b}-\alpha\right) \\
& \frac{\partial^{2} \Omega}{\partial \alpha \partial \beta}=\tilde{\mathrm{f}}_{0} \frac{2}{\beta^{3}} I\left(-\beta V_{b}-\alpha\right)+\tilde{\mathrm{f}}_{0} \frac{V_{b}}{\beta^{2}} L\left(-\beta V_{b}-\alpha\right)
\end{aligned}
$$

The derivatives with respect to $\vec{\gamma}$ can be evalueted by using the following trick. Introduce a "temperature in z-direction" as $\beta_{z}$, then define

$$
\hat{\Omega}\left(\alpha, \beta, \beta_{z}\right)=\tilde{\mathrm{f}}_{0} \int_{\mathrm{V}_{0}}^{\infty} \mathrm{d} \varepsilon_{z} \int_{0}^{\infty} \mathrm{d} \varepsilon \ln \left[1+e^{-\beta \varepsilon-\beta \varepsilon_{z}-\alpha}\right\}
$$

which is related to $\Omega$ by

$$
\Omega(\alpha, \beta, \vec{\gamma}=0)=\hat{\Omega}(\alpha, \beta, \beta)
$$


The coordinate transformation $x=-\beta \varepsilon+y$ and $y=-\beta_{z} \varepsilon_{z}-\alpha$, which for $\beta=\beta_{z}$ has led to eq. $(3.98)$, now leads to

$$
\hat{\Omega}\left(\alpha, \beta, \beta_{z}\right)=\widetilde{f}_{0} \frac{1}{\beta \beta_{z}} K\left(-\beta_{z} v_{b}-\alpha\right)
$$

From

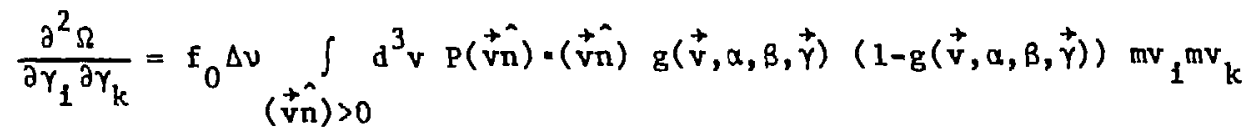

one realfzes for $\stackrel{+}{y}=0$ that due to axial symmetry

$$
\frac{\partial^{2} \Omega}{\partial \gamma_{x}^{2}}=\frac{\partial^{2} \Omega}{\partial \gamma_{Y}^{2}} \quad \text { if } \hat{n} \text { denotes the } z \text {-direction }
$$

and fur thermore

$$
\left.\frac{\partial^{2} \Omega}{\partial \gamma_{x}^{2}}\right|_{0}+\left.\frac{\partial^{2} \Omega}{\partial \gamma_{y}^{2}}\right|_{0}=\left.m \frac{\partial^{2} \hat{\Omega}}{\partial \alpha \partial \beta}\right|_{B_{z}=\beta_{0}}=2 m \tilde{f}_{0} \frac{1}{\beta_{0}^{3}} I\left(\beta_{0}\left(\varepsilon_{F}-V_{b}\right)\right)
$$

Herewith, we get for the tangential part

$$
\left.\frac{\partial^{2} \Omega}{\partial \gamma_{\mathbf{x}}{ }^{2}}\right|_{0}=\left.\frac{\partial^{2} \Omega}{\partial \gamma_{\mathbf{y}}{ }^{2}}\right|_{0}=\mathrm{m} \tilde{\mathrm{f}}_{0} \frac{1}{\beta_{0}{ }^{3}} I\left(\beta_{0}\left(\varepsilon_{\mathrm{F}}-\mathrm{V}_{\mathrm{b}}\right)\right)=-\left.\mathrm{m} \frac{1}{\beta_{0}} \frac{\partial \Omega}{\partial \alpha}\right|_{0}
$$

Since $\left.B_{0} \frac{\partial^{2} \Omega}{\partial \gamma^{2}}\right|_{0}$ is the friction coefficient one sees from eq. (3.4.40) and from eq. (3.4.38) that the tangential window friction coefficient is equal to the one-sided current of particles through the window. The longitudinal coefficient can he calculated by using the identity 
as

$$
\frac{\partial^{2} \Omega}{\partial \gamma_{x}^{2}}+\frac{\partial^{2} \Omega}{\partial \gamma_{y}^{2}}+\frac{\partial^{2} \Omega}{\partial \gamma_{z}^{2}}=2 m \frac{\partial^{2} \Omega}{\partial \alpha \partial \beta}
$$

$$
\left.\frac{\partial^{2} \Omega}{\partial Y_{z}^{2}}\right|_{0}=\left.2 m \frac{\partial^{2} \Omega}{\partial \alpha \partial \beta}\right|_{0}-\left.2 m \frac{\partial^{2} \hat{\Omega}}{\partial \alpha \partial \beta}\right|_{\beta_{z}=\beta}
$$

which yields

$$
\left.\frac{\partial^{2} \Omega}{\partial Y_{z}^{2}}\right|_{0}=2 m \tilde{f}_{0} \frac{1}{\beta_{0}^{3}}\left[I\left(\beta_{0}\left(\varepsilon_{F}-V_{b}\right)\right)+\beta_{0} V_{b} L\left(\beta_{0}\left(\varepsilon_{F}-V_{b}\right)\right)\right]
$$

The longitudinal friction coefficient derived here is, in general, not just twice the tangential one as in [BB78] but contains an additional part. However, this part vanished for $v_{b}=0$.

The cross terms $\partial^{2} \Omega /\left.\partial \gamma_{1} \partial \gamma_{k}\right|_{0}$, where $i \neq k$, vanish due to the axial symmetry when $\hat{n}$ is the z-axis. This is easily verified by using eq. $(3.4 .46)$. Since the functions $I(x)$ and $K(x)$ do not exist in an analytic form, it is quite convenient to use the following approximations:

$$
I(x) \approx \frac{\pi^{2}}{12}\left[1+\operatorname{sgn}(x) \frac{1-\exp \left(\frac{-24 \ln 2}{\pi^{2}} x\right)}{1-\exp (-.955 x)}\right]+\theta(x) \frac{x^{2}}{2}
$$

The deviation from the exact function is not greater than $3.10^{-4}$ over the whole range $-\infty<x<+\infty$.

$$
K(x)=x I(x)+K_{0}\left[\frac{\exp (-.766 x)-\exp (-2 \alpha x)+2 \exp (-\alpha x)}{1+\exp (-.766 x)}\right]-\theta(x) \frac{x^{3}}{3}
$$

with $K_{0}=\sum_{\nu=1}^{\infty} \frac{(-1)^{\nu+1}}{v^{3}}=.90154268$ and $\alpha=\sqrt{\frac{1 \mathrm{n} 2}{K_{0}}}=.87683845$. The maxima 1 deviation for this approximation is $9 \cdot 10^{-4}$ for all $\mathrm{x}$. 
The window friction coefficients derived in [BB78] were calculated for zero temperature and no barrier which means $\beta_{0}\left(\varepsilon_{F}-v_{0}\right) \rightarrow \infty$. We retrieve the same coefficients by taking this limit and recognizing the behavior of $L(x), I(x)$ and $K(x)$ for large $x$ which is given by

$$
\begin{aligned}
& L(x>>1)+x \\
& I(x>>1)+\frac{x^{2}}{2}+\frac{x^{2}}{6} \\
& L(x>>1)+\frac{x^{3}}{6}+\frac{\pi^{2}}{6} x .
\end{aligned}
$$

The next step is to relate the coefficients calculated for semiInfinite nuclear matter to those for the actual shape. To do this we make use of the proximity 1dea. Given a shape as indicated in fig. 3.4 .6 we assume that the fluxes at each radial distance $\nu$ are equal to those calculated for the case of plane surfaces. Therefore, the coefficients appearing in eq. $(3.4 .28)$ can be calculated by integrating those given in eqs. $(3.4 .29)$ through (3.4.32) along the radial coordinate $v$ :

$$
g_{a b}(q)=\int_{0}^{\nu} \frac{2 \pi \nu \cdot d \nu}{\Delta \nu} g_{a b}(D(\nu)) ; a, b=A, s, x, y, z
$$

The area element $\Delta v$ is now $2 \pi u d \nu$. The upper boundary is equal to the radius of the smaller sphere $\nu_{\max }=\min \left\{R_{1}, R_{2}\right\}$. 


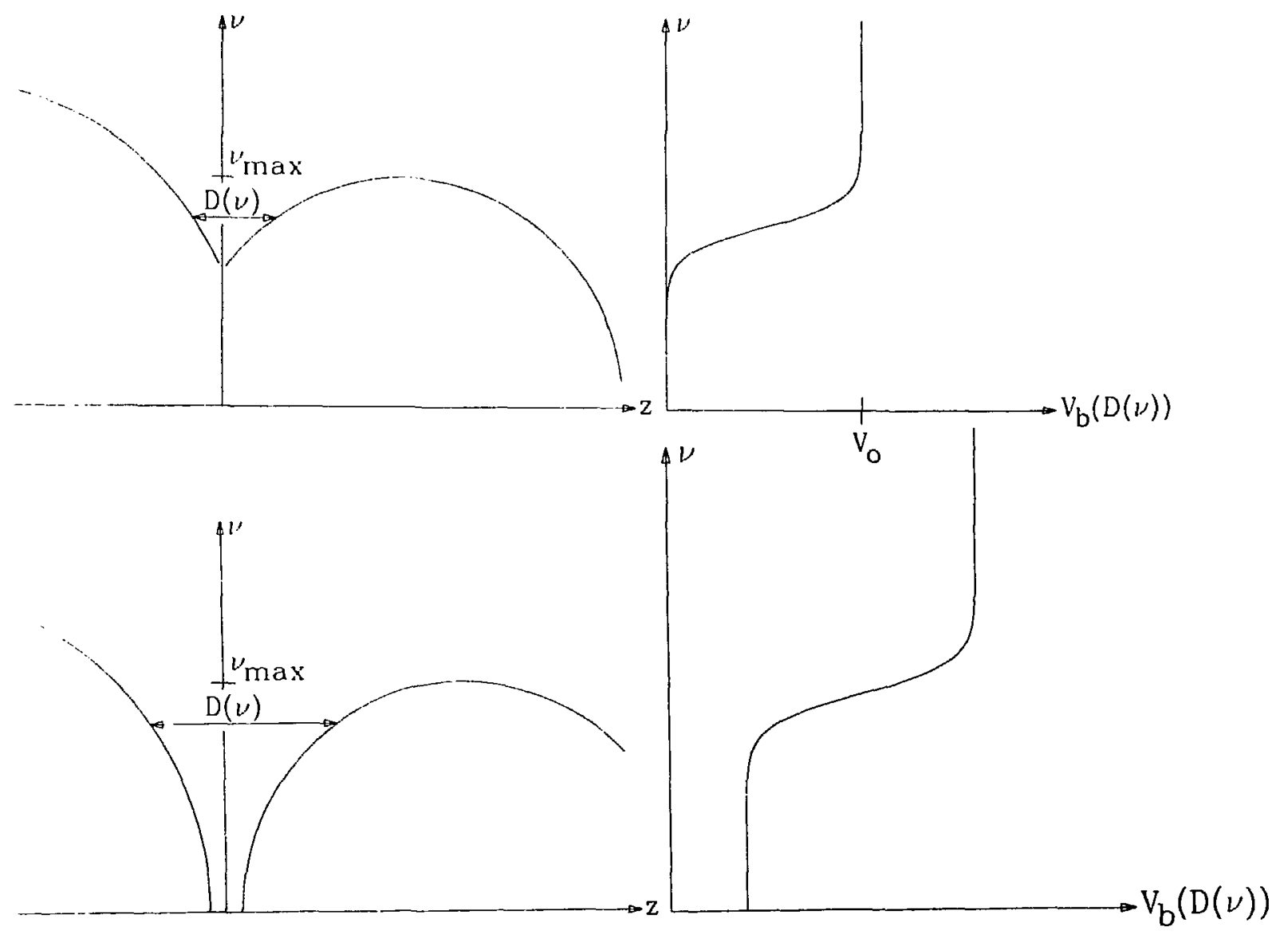

Fig. 3.4.6 Potential barrier in the vicinity of the geometricai window (upper) and for separated shapes (lower).

The coefficlents $g_{a b}(D(v))$ depend on the barrier height $\nabla_{b}(D)$ which depends on the separation between the two nuclear surfaces $D(v)$; this distance is a unique function of the shape coordinates $q=\{s, \sigma, \Delta\}$. Therefore, the integrated coefficients $g_{a b}(q)$ depeno on the actual shape. They also depend on the mean temperature. In the general case the geometrical window which is completely open $\left(v_{b}=0\right)$ is surrounded by a corona which has an increasing potential barrier $V_{b}(D)$. For separated shapes we encounter a proximfty effect in the friction and in the flux of particles and heat even before the nuclei touch with thes: equivalent sharj surfaces (c.f. fig. 3.4.6). 
Herewith, we have calculated within a unified microscopic model, transport coefficients for four difflerent phenomena:

1. the gradient in chemical potential, $\Delta \mu$, drives the particle flux,

2. the temperature mismatch, $\Delta T$, drives the entropy flux,

3. the velocity mismatch, $\overrightarrow{\Delta u}$, sets in motion the momentum flux,

4. the coupling between particle and entropy flux drives particles from the hotter gas to the colder gas.

The flux of particles from the hotter gas to the colder gas counteracts the driving force towards symmetry in mass number, as has been pointed out by Moretto [Mo83]. In how far 1 t can be made responsible for the lack of mass drift seen in heavy ion collisions will be discussed later.
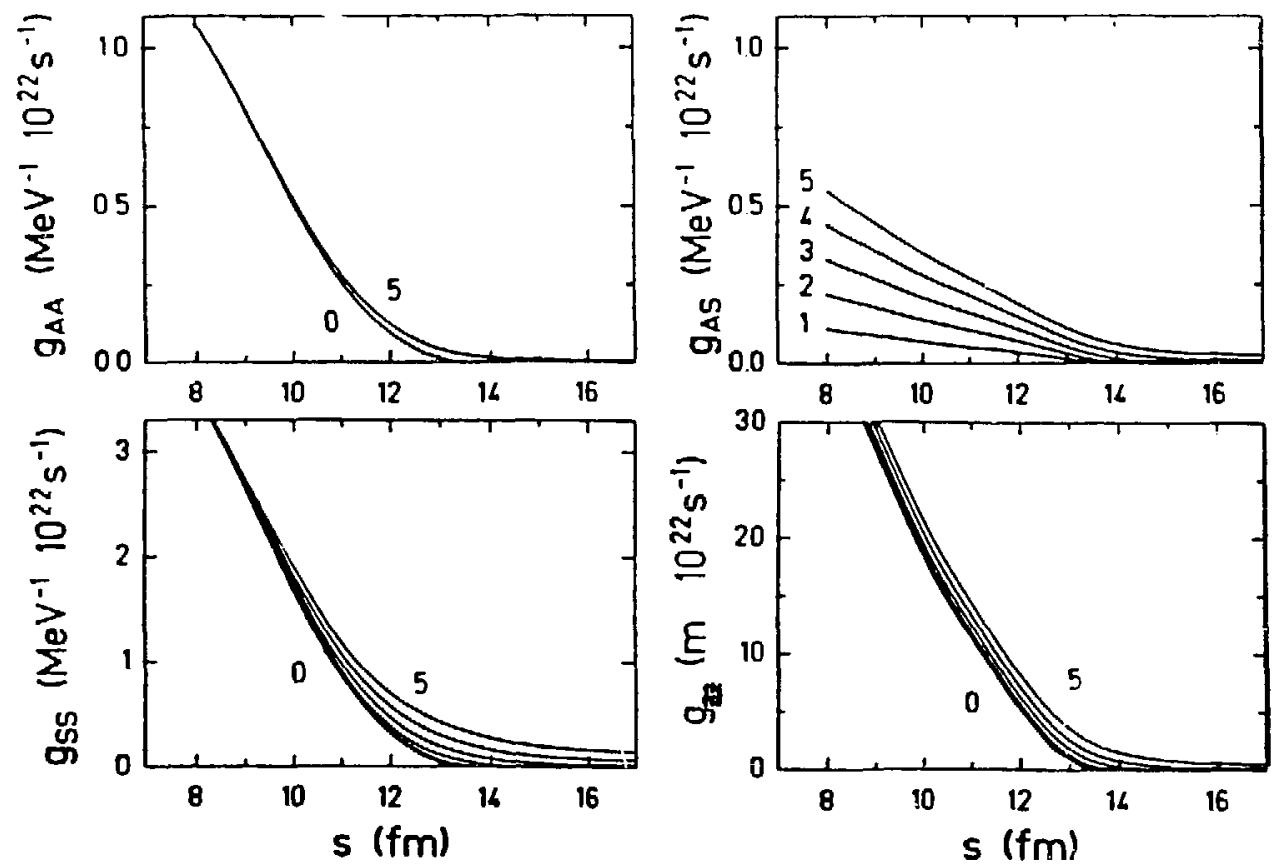

Fig. 3.4.7 Dependence of the different transport coefficlerts on the distance 8 , between $H o$ and $F e$ and on the temperature. The curves are labeled by the temperature in MeV. The mass of a nucleon is denoted by $\mathrm{m}$. 
In fig. 3.4.7 the dependence of the coefficients on the distance $s$ and the mean temperature $T$ is shown for Fetho $(\sigma=0, \Delta=0.156)$. The two spheres touch at $s_{t}=11.1 \mathrm{fm}$. The mobility coefficient $g_{\mathrm{AA}}$ exhibits the weakest temperature dependence, whereas the cross coeffirient $g_{A S}$ is almost linear in T. This can also be seen from the explicit expressions $(3.4 .30),(3.4 .40)$, (3.4.42) and (3.4.55). For zero temperature the longitudinal coefficient $g_{33}$ is almost the same as in Randrup's proxtmity friction because we have chosen the parameterization for the potential barrier $V_{h}(D)$ such that tine corresponding universal function is as close as possible to Randrup's universal function. The shape dependence of: the other coefficients is thereby determined. It is also worthwhile to note that for a completely open window $\left(v_{b}=0\right)$ and zero temperature, $1 / g_{A A}$ becomes the friction coefficient which has been discussed recently be Swiatecki and Randrup to complete the wali flus window friction [RS84]:

$$
1 / \mathrm{g}_{\mathrm{AA}}=4 \mathrm{p}_{\mathrm{F}} /\left(3 \rho \mathrm{a}_{\mathrm{W}}\right)
$$

where $\rho$ is the particle density, $p_{F}$ the Fermi momentum and $a_{w}$ the window area. A typical value for this coefficient is $.5 \mathrm{MeV}^{-1} 10^{22} \mathrm{~s}^{-1}$ (c.f. $\mathrm{fig}$. (3.4.7)) which means that for a typical mismatch in chemical potentials of $\Delta \mu=1 \mathrm{MeV}$, the change in the mean number of particles per unit time is of the order of

$$
\dot{A}_{1}=g_{A A} \Delta \mu=5 / 10^{-21} s
$$

For collisions which end up with final energies above the Coulomb barrier the typical reaction time is $10^{-21} \mathrm{~s}$. This means that the mean mass drift is only a few mass units for these reactions or in other words, the mobility of 
nucleons through the window 18 rather small. These simail mass drifts are typical for pertpheral heavy ion collisions. A more detalled discussion of the mass drift and how it is influenced by the thermal force ${ }_{A S} \Delta T$ will follow when we look at the results of trajectory calculations.

In this section the mass number $A_{1}$ of one nucleus appears as a macroscoplc variabie rather than the asymmetry $\Delta$ which we introduced in section 3.1. For shapes with a thin neck $A_{1}$ can be uniquely related to the variable $\Delta$ by

$$
A_{i}=A \frac{(1+\Delta)^{2}}{1+3 \Delta^{2}}
$$

where $A$ is the total mass number of the system. For shapes without neck we shall use the same relation to define $A_{1}$. The problems arising from this will be discussed in subsection 3.4.3. Thus, whereever $A_{1}$ appears it may be replaced by the macroscopic variable $\Delta$. For example, the heat produced per unit time by particle exchange is given by

$$
\dot{Q}^{\text {window }}=\sum_{a, b=\{x, y, z\}} \Delta_{a} g_{a b} \Delta u_{b}+\dot{A}_{1} \frac{1}{g_{A A}} \dot{A}_{1} \text {. }
$$

In this equation we have to express the velocity mismatch $\Delta$ and the mass number $A_{1}$ in terms of the macroscopic variasles:

$$
\begin{aligned}
& \Delta u_{a}=\sum_{\ell} C_{a \ell}^{\mathrm{m}}(q) \dot{q}_{\ell} \\
& \dot{A}_{1}=\sum_{\ell} c_{\ell}^{A}(q) \dot{q}_{\ell}=\frac{d A_{1}}{d \Delta}(\Delta) \dot{\Delta} .
\end{aligned}
$$


The coefficients $c_{i \ell}^{m}(q)$ which relate the mismatch in velocity to the macroscoplc varlables will be worked out later. Using eqs. (3.4.61) and $(3.4 .62)$ we can write down the window friction tensor $R^{\text {window }}$ as

$$
R_{1 k}^{w 1 n d o w}=\sum_{a, b=\{x, y, z\}} C_{1 a}^{m} g_{a b} c_{b k}^{m}+\left(\frac{d A}{d \Delta}\right)^{2} \frac{1}{g_{A A}} \delta_{33}
$$

where $\Delta=q_{3}$. The heat produced by the window friction can be summarized by

$$
\dot{Q}^{\text {indow }}=\sum_{1, k=1}^{6} R_{1 k}^{\text {wlndow }} \dot{q}_{1} \dot{q}_{k}
$$

However, one should keep in mind that the particle flux Induced by the temperature difference reduces the heat production by an amount

$$
\dot{\mathrm{Q}}^{\Delta \mathrm{T}}=-\dot{\mathrm{A}}_{1} \frac{\mathrm{g}_{\mathrm{AS}}}{\mathrm{g}_{\mathrm{AA}}} \Delta \mathrm{T}
$$

This quantity is negative since $\dot{A}_{1}$ has the same sign as $\Delta T=T_{2}-T_{1}$ and the coefficlents $g_{A S}$ and $g_{A A}$ are positive.

\subsubsection{Window-plus-wa 11 and wall dissipation.}

In this section we combine the wall and the window dissipation. In order to do so we have to distinguish between di-nuclear and mono-nuclear shapes. Let us flrst consider the two extreme cases for which the distinction 1s clear. Shapes for which the window area between the two nuclei is small compared to the surface are called dinnuclear shapes. Mono-nuclear shapes do not have a neck and are more or less convex objects. Fig. 3.4.8 shows a family of shapes and Indicates the divfsion Into mono- and di-nuclear shapes. 
Let us first consider the case of di-nuclear shapes. If we combine for those the wall dissipation discussed in section 3.4 .1 with the window dissipation as derived in the previous section 3.4 .2 we then get a damplng mechanism which acts on all degrees of freedom (eqs. $(3.4,11)$ and $(3.4 .64)$ ) called the window-plus-wall dissipation:

$$
\dot{Q}^{w+w}=\dot{Q}^{\text {window }}+\dot{Q}_{\mathrm{d} 1}^{\text {wal1 }}
$$

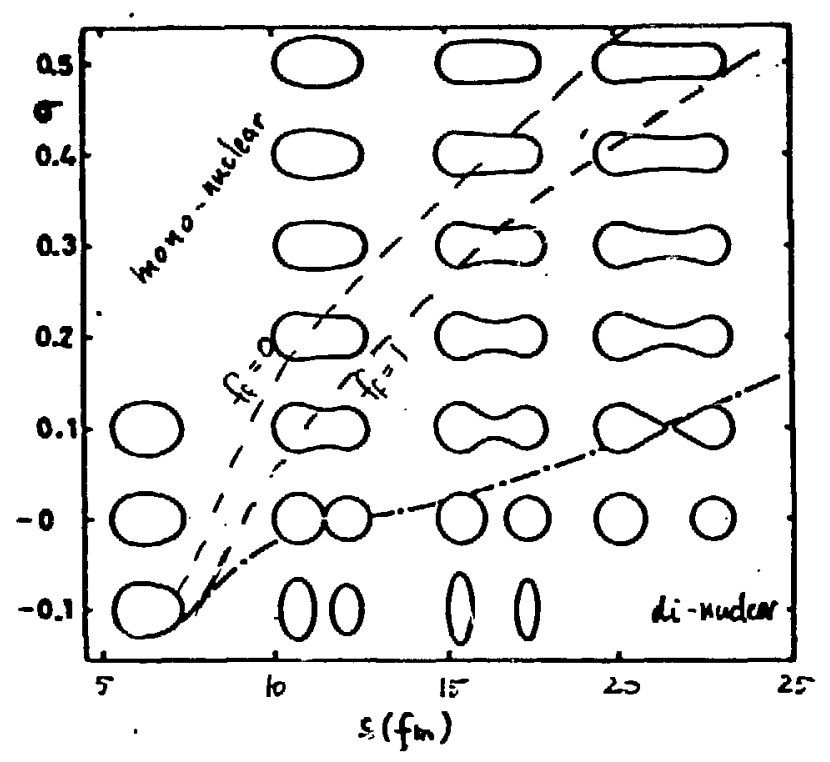

Fig. 3.4.8 Form factor for the transition from di-nuclear to mononuclear shapes.

However, we ought to be cautious not to double count to a certain extent. This problem arises because, in the case of window friction, the randomization hypothesis should be achleved malnly by successive collistons with the wall of the acceptor vesse1. On the other hand, the wall is the self-consistent potential which does not move by 1 ts own but rather reflects 
the bulk motion of the gas inside. Therefore, we have to use two different drift velocities $\vec{v}_{\mathrm{d} 1}$ and $\dot{\mathrm{v}}_{\mathrm{d} 2}$, one for each nucleus. Furthermore, the velocity mismatch in the longitudinal direction, $\hbar_{u} \cdot \hat{n}$ should be the difference between $v_{d 2}$ and $v_{d 1}$. In this way we avoid spurious contributions and take into accont the self-consistency of the wall motion as far as possible.

The cholce of the drift velocities $\vec{v}_{\mathrm{d} 1}$ and $\overrightarrow{\mathrm{v}}_{\mathrm{d} 2}$ is still an open question. Let us consider the problem in a co-rotating coordinate frame in which the z-axis is the symmetry axis of the shape. The first assumption is that the drift velocity field is uniform. . Then the axial symmetry implies that $\vec{v}_{\mathrm{dl}, 2}$ has only a z-component. The drift velocity fields are divided by a plane at position $z_{d}$ which, for connected shapes, is defined by

$$
A_{1}=\rho \int_{z_{0}}^{z} d P^{2}(q ; z) d z
$$

For separated shapes $z_{\mathrm{d}}$ is defined to be as

$$
\mathrm{z}_{\mathrm{d}}=\frac{\mathrm{R}_{1}}{\mathrm{R}_{1}+\mathrm{R}_{2}} \mathrm{~s}
$$

This means that there are $A_{1}$ particles left of $z_{d}$ and $A-A_{1}$ particles on the right. $A_{1}$ is related to the asymmetiy degree of freedom $\Delta$ by eq. (3.4.59). The drift velocities $\vec{v}_{\mathrm{d} 1}$ and $\overrightarrow{\mathrm{v}}_{\mathrm{d} 2}$ are determined such that no heat $1 \mathrm{~s}$ produced for pure translational and rotational motion of the shape. The latter has been eliminated already because we are working in the co-rotating frame. For the translational motion we utilize the idea of minimal entropy production. This means that we are choosing the drift velocities $\vec{v}_{\mathrm{d} 1,2}$ of the drift velocity field 


$$
v_{d}(z)=\sum_{1}\left\{\Phi_{11} \theta\left(z_{d}-z\right)+\Phi_{21} \theta(z-z d)\right\} \dot{q}_{1}
$$

such that the heat produced by the wall is minimized:

$$
\frac{\partial \dot{Q}_{d 1}^{\text {wa } 11}}{\partial \Phi_{11}}=0, \frac{\partial \dot{Q}_{d 1}^{* \text { wall }}}{\partial \Phi_{21}}=0
$$

Looking back at eq. $(3.4 \cdot 10)$ this leads to

$$
\phi_{1 i}=-\int_{z_{0}}^{\zeta_{1}} \frac{\frac{\partial p^{2}}{\partial q_{1}} \frac{\partial p^{2}}{\partial z}}{d\left(P^{2}+\frac{1}{4}\left(\frac{\partial P^{2}}{\partial z}\right)^{2}\right)} d z\left[\int_{z_{0}}^{\zeta} \frac{\frac{\partial p^{2}}{\partial z}}{\sqrt{ }\left(P^{2}+\frac{1}{4}\left(\frac{\partial P^{2}}{\partial z}\right)^{2}\right)} d z\right\}^{-1}
$$

For $\Phi_{21}$ the integration has to be performed from $\zeta_{2}$ to $z_{5}$ (c.f. subsection 3.1); $\zeta_{1,2}=z_{d}$ for connected shapes and $\zeta_{1}=z_{2}, \zeta_{2}=z_{3}$ for separated shapes. The components $\Phi_{11}, \Phi_{21}$ of the drift velocity field have the following properties. For a pure translation of one or the other part of the separated shape there will be no wall friction at all. For connected dinuclear shapes a motion in the $s$ degree of freedom gives only little wall dissipation because the walls are still making more or less a translational motion. If calculated for each part of the separated shapes individually, the drift velocities coincide with the drift velocity proposed by Swlatecki [Sw80].

To be consistent, we should also use the above determined drift velocities for the mismatch velocity occuring in the window dissipation, name ly

$$
\overrightarrow{\Delta \vec{u} \cdot \hat{n}}=\sum_{1}\left(\Phi_{2 i}-\Phi_{11}\right) \dot{q}_{1}
$$


Mono-nuclear shapes are encountered for example in the case of induced fission of a heavy nucieus where the trajectory starts at the saddle point of a rather compact shape. Or one could Imagine that a heavy ion collision has produced a rather compact compound-like nucleus which subsequently fissions due to 1 ts high fissility. Looking at those mono-nuclear shapes in fig. 3.4 .8 , one would rather use a unfform drift velocity field for the whole shape as

$$
v_{d}=\sum_{i} \Phi_{m i} \dot{q}_{i}
$$

This drift velocity can be obtained from eq. (3.4.71) by setting the upper boundary $\zeta_{1}=z_{5}$, which means an integration over the whole shape. The window has disappeared in mono-nuclear shapes so that the disalpation is only due to wall friction. The rate of disslpated energy per unit time can be calculated from eqs. $(3.4 .10)$ and $(3.4 .11)$ by using the drift velocity given in eq. $(3.4 .73)$ :

$$
\dot{Q}_{\text {mono }}^{\text {wall }}=\sum_{1, k}\left(\mathrm{R}_{\text {mono }}^{\text {wall }}\right)_{1 k} \dot{q}_{1} \dot{q}_{k}
$$

Since up to now there is no scheme based on a microscopic picture which would allow a smooth transition between the mono- and the di-nuclear regime, we simply introduce a form factor $f_{f}$ which allows a smooth transition between the two dissipation mechanisms.

$$
\dot{Q}=\left(1-f_{f}\right) \dot{Q}^{w+w}+f_{f} \dot{Q} \underset{m o n o}{\text { wall }}
$$

The form factor $f_{f}$ is defined as: 


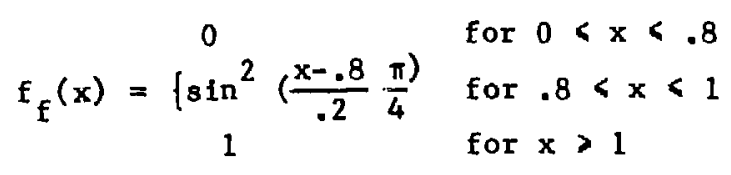

where

$$
x= \begin{cases}0 & \text { for separated shapes } \\ R_{\text {neck }}^{2} / \min \left\{R_{1}^{2}, R_{2}^{2}\right\} & \text { for shapes with neck }\end{cases}
$$

The quantity $x$ which enters eq. $(3.4 .76)$ is a measure of the ratio between the window area and the wall area surrounding a particle in the smaller part of the shape. At $x=R_{\text {neck }}^{2} / R_{1}^{2}=1$ the probability that the particle will go through the window rather than hit the wall is about $1 / 3$. This corresponds to a situation where the neck is gone and there is no minimum anymore in the profile function $P(z)$. From here on we regard these shapes to belong to the mono-nuclear reglme $\left(f_{f}=1\right)$. For $x<.8$, which means a probability of less than about $20 \%$ to escape through the window, we assume that the re will be a thermal equilibrium within each side of the shape so that we are dealing with the di-nuclear situation described in subsection 3.4.2. Here, we use the window-plus-wall dissipation. In between, the form factor $f_{f}$ smoothly jolns the two dissipation mechanisms. This construction is certainly not satisfying and one may argue about the form factor $f_{f}[N I 84, B S 82]$ or the whole procedure. Especially for very elongated shapes the decision between the mono- and the di-nuclear regime is difficult. In order to get a feeling in how far $\dot{Q}^{W+w}$ and $\dot{Q}_{\text {mono }}^{\text {wall }}$ differ for the same shape let us consider the simple case of a cylinder which stretches in the direction of the symmetry axis while shrinking in the radial direction in order to conserve the total volume. The geometry is illustrated in fig. 3.4.19. 


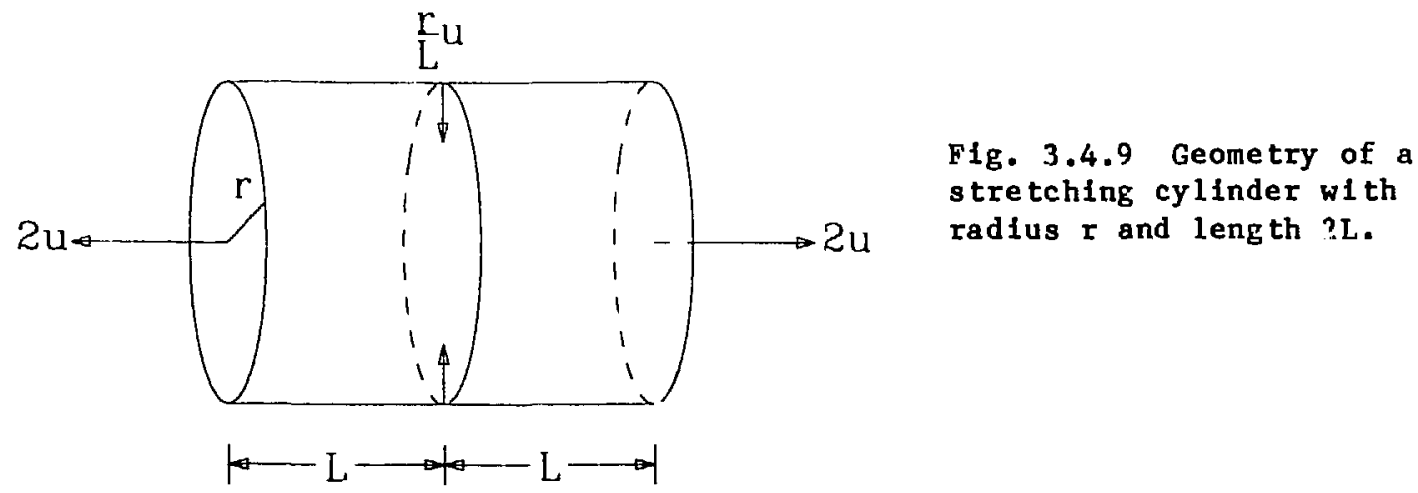

In the mono-nuclear case the drift velocity is zero and we get for the rate of dissipated energy

$$
\dot{Q}_{\text {mono }}^{\text {wall }}=m \rho \overline{v \pi r}\left(8+4 \frac{r}{L}\right) u^{2}
$$

If we regard the cylinder as a di-nuclear shape the two drift velocities are $v_{\mathrm{dl}, 2}= \pm 2 u$ which gives to a window-plus-wall dissipation equal to

$$
\begin{aligned}
\dot{Q}^{\text {window }}+\dot{Q}_{\mathrm{d} 1}^{\mathrm{wall}} & =m \frac{\rho \overrightarrow{\mathrm{v}}}{4} \pi \mathrm{r}^{2} 2(4 \mathrm{u})^{2}+\mathrm{m \rho} \overline{\mathrm{v}} \pi \mathrm{r}^{2} 4 \frac{\mathrm{r}}{\mathrm{L}} \mathrm{u}^{2} \\
& =m \rho \overline{\mathrm{v}} \pi \mathrm{r}^{2}\left(8+4 \frac{\mathrm{r}}{\mathrm{L}}\right) \mathrm{u}^{2}
\end{aligned}
$$

It turns out to be just the same result as that for the mono-nuclear case. This amazing outcome is a frieno-y gesture of the one-body dissipation which relaxes somewhat the concern on how to choose the form factor $f_{f}$. Also the 
friction tensor for the shapes which we are using shows the same behavior. For the stretching degree of freedom window-plus-wall and wall dissipation give simflar results.

However, this does not apply to the asymmetry degree of freedom. Let us consider a change of the shape, Indicated in fig. 3.4.10 where one half shrinks and the other half blows up in the radial direction. The drift velocities vanish in this configuration.

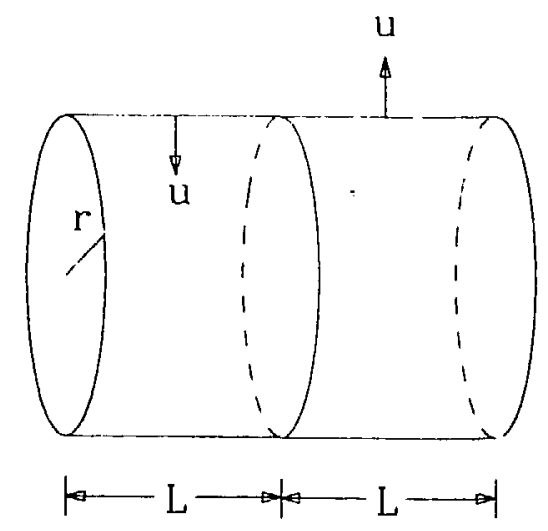

Flg. 3.4.10 Geometry of a cylinder which is changing the asymmetry.

The mono-nuclear dissipation is given by

$$
\dot{Q}_{\text {mono }}^{\text {wall }}=m \rho \bar{v} 2(2 \pi r L) u^{2}=m \rho \bar{v} \pi u^{2} 4 r L
$$

In the window-plus-wall case, we obtain

$$
\begin{aligned}
\dot{Q}_{\text {momo }}^{\text {wa } 11}+\dot{Q}_{\mathrm{d} i}^{\text {wall }} & =\dot{Q}^{\text {window }}+\dot{Q}_{\text {mono }}^{\text {wa } 11} \\
& =\frac{4}{3} \frac{\mathrm{mv}_{\mathrm{F}}}{\rho \pi \mathrm{r}}(\rho \mathrm{L} 2 \pi \mathrm{ru})^{2}+\mathrm{m \rho v} \pi \mathrm{u}{ }^{2} 4 \mathrm{rL}
\end{aligned}
$$


Here, we used eq. $(3.4 .60)$ for $\dot{Q}^{\text {window }}$ and eq. $(3.4 .57)$ for the friction coefficient $\left(\Delta \vec{u}=\vec{v}_{\mathrm{d} 2}-\vec{v}_{\mathrm{d} l}=0\right)$. The mono- and di-nuclear wall dissipation rates are equal in this example because all drift velocities are zero. The final result

$$
\dot{Q}^{\text {window }}+\dot{Q}_{d i}^{\text {wall }}=m \rho \bar{v} \pi u^{2}\left(\frac{64}{9} L^{2}+4 L \tau\right)
$$

(using $\bar{v}=\frac{3}{4} v_{F}$ ) shows that for elongated shapes the dissipation rate is quite different. For $\mathrm{L}=\mathbf{2 r}$ the window-plus-wall nechanism produces a 4.6 times stronger friction against a change of the mass asymetry than the mono-nuclear wal1 dissipation! 
3.5 Fluctuations caused by the exchange of particles

In this section we will derive the friction and diffusion

coefficients for the particle exchange picture following the treatment of Brownian motion as presented 1 in sections 1 and 1.1 . From the quite general equations (1.1.17) and (1.1.29) we saw that friction force (drift $c_{\sim}$ fficient) and diffusion tensor are first and second moments of the same probability distribution $w(\vec{p}, \vec{q} \mid \vec{n}, \tau)$ which represents one form of the so-called dissipation fluctuation theorem. Of course we expect the friction force to be identical to the result of section 3.4 .2 where we did not discuss fluc tuations.

We will determine explicitly $W(\vec{p}, \vec{q} \mid \vec{n}, \tau) d^{3} \eta$, which is the conditional probability that the relative momentum of the di-nuclear system has changed from $\vec{p}$ to $\stackrel{+}{p}+\vec{n}$ due to the action of internal degrees of freedom during the time $\tau$.
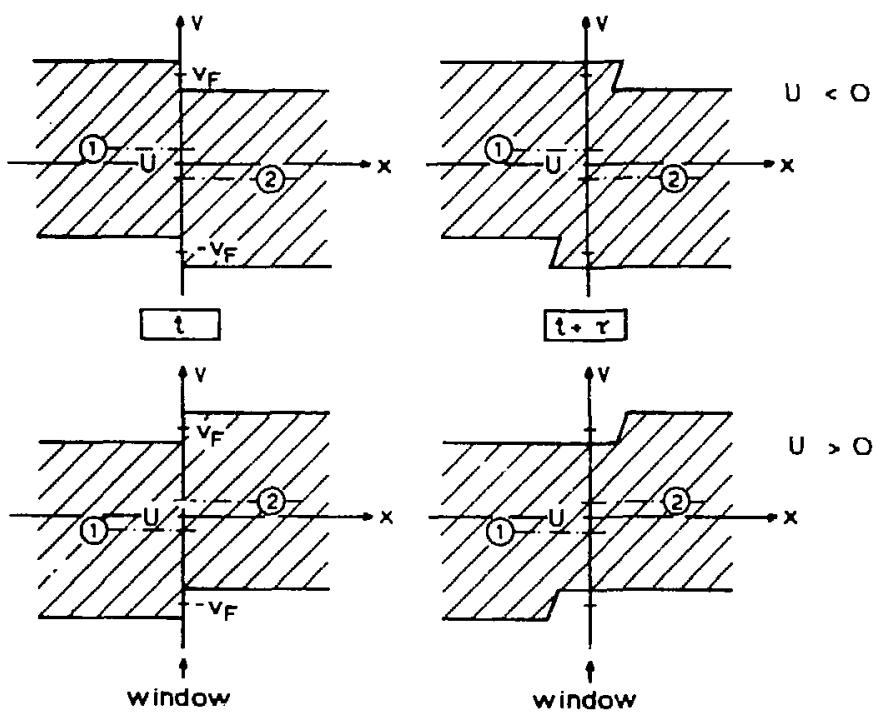

Fig. 3.5.1 Illustration of the window dissipation in the one dimensional one-particle phase space. Upper part: approaching Fermi gas. Lower part: diverging gases. The hatched areas indicate the occupled part. 
The first and second moments with respect to $\vec{n}$, will be calculated under certain approximations. We are going to consider the exchange of particles between the two nuclei as a dissipative mechanism ("window dissipation"). There are certainly other means of dissipation in heavy-ion collisions, but in order not to blur the effects by adding different sources of fluctuations we regard here particle exchange only.

For illustrative purposes, it is quite useful to consider first the window dissipation for a one-dimensional knudsen gas. Similar to the discussion of the wall friction in subsection 3.4 .1 we show in fig. 3.5 .1 the one-body phase-space distribution for two Fermi-gases in contact at a window $(x=0)$. Their mean velocities are shifted with respect to each other by $u=$ $u_{2}-u_{1}$, and there is no potential barrier in this example. In the upper part of fig. 3.5.1 we display the situation for approaching gases at itme $t$ and $t+\tau$. Due to the mismatch in velocities, occupied cells (hatched area) from the left enter through the window into unoccupied phase-space on the righthand side and vice-versa. As expected, when calculating the mean velocity on one side at time $t+\tau$, it has decreased when compared to time $t$. The same holds if the two gases are diverging as illustrated in the lower part of $\mathrm{fig}$. 3.5.1. Here, empty phase-space cells are moving into criginally occupied space. This exchange of holes has the same dissipative effect as the exchange of particles. In the fnterior region fully occupied cells also cross the window replacing other occupied cells which themselves have moved away. However, this is only a rearrangement in the occupled phase space and does not result in an observable change in the state of the system. Therefore, only transitions in the little step-like edges close to the Fermi velocity, $v_{F}$, contribute to a change in observable quantities such as the relative momentum or the mass number of one nucleus. 
Let us denote with $g_{1,2}(\vec{v})$ the Fermi-distribution of the velocities in nucleus 1 and 2 , respectively, where

$$
\Sigma_{1}(\vec{v})=\left[1+\exp \left\{\frac{1}{2} m\left(\left(\vec{v}-\vec{u}_{i}\right)^{2}-v_{F}^{2}\right) / T_{i}\right\}\right]^{-1}, \quad i=1,2 .
$$

Here, $\vec{u}_{i}$ denotes the mean velocity and $T_{i}$ the temperature of nucleus $i=1,2$. For reasons of simplicity we are not going to treat the mass drift here; therefore we assume the same Fermi-velocity, $v_{F}$, for both nuclei. Looking at Fig. 3.5.1 it becomes evident that the number of particles with velocity between $v$ and $v+d v$ which have been exchanged from right to left inco previously unoccupled phase space is glven by

$$
\pi_{I \rightarrow 2}(v) d v=f_{0} \theta(v) v \tau g_{1}(v)\left(1-g_{2}(v)\right) d v
$$

For small $\tau$ this is also the probability that a particle with velocity between $v$ and $v+d v$ has been transierred from gas 1 to gas 2 . In the six-dimensional phase-space the analogue is

$$
\Pi_{1+2}(\vec{v})=f_{0} P_{b}(\vec{v}, \vec{v}) \theta(\vec{v} \hat{n})(\vec{v} n) \Delta v \tau g_{1}(\vec{v})\left(1-g_{2}(\vec{v})\right)
$$

Here, the phase-space density of nuclear matter is taken as $f_{0}=3 /\left(4 \pi r_{0}^{3}\right) 3 /\left(4 \pi v_{F}^{3}\right)$, where $r_{0}=1.18 \mathrm{fm}$ is the radius parameter and $v_{F}=7.84 \times 10^{22} f_{\text {in }} s^{-1}$ is the Ferm 1 veloctty. The window-area element and 1 ts direction are denoted by $\Delta \nu$ and $\hat{n}$, respectively, while $\theta\left(v^{*}\right)$ is the step function which allows only for those particles which have a positive velocity component in the window direction. The probability for a particle having a velocity $\vec{v}$ to cross the barrier at a location denoted by $\vec{v}$ is given by the penetrability $\mathrm{F}_{\mathrm{b}}(\vec{v}, \vec{v})$. 
The probability per velocity bin that a hole moves from gas 1 to gas

2 is given by:

$$
\tilde{\Pi}_{1,2}(\vec{v})=E_{0} P_{b}(\stackrel{\vec{v}}{\vec{v}}) \theta(\vec{v} \hat{n})(\vec{v} n) \Delta \nu \tau g_{2}(\vec{v})\left(1-g_{1}(\vec{v})\right) .
$$

The analogue single-particle transition probabilities for nucleus 2 are:

$$
\begin{aligned}
& \left.\Pi_{2+1}(\vec{v})=f_{0} P_{b}(\vec{v}, \vec{v}) \theta(-\vec{v})(-\stackrel{+}{n}) \Delta \nu \tau g_{2}(\vec{v}), 1-g_{1}(\vec{v})\right) \\
& \tilde{\Pi}_{2 \rightarrow 1}(\vec{v})=f_{0} P_{b}(\vec{v}, \vec{v}) \theta(-\vec{v} \hat{n})(-\vec{v} \hat{n}) \Delta \nu \tau g_{1}(\vec{v})\left(1-g_{2}(\vec{v})\right) .
\end{aligned}
$$

The velocities ir eqs. (3.5.3) - (3.5.6) are with respect to the rest frame of the window. Since it is assumed that the mass drift vanishes and that the Fermi velocities for both nuclei are equal, the window velocity is the mean, $\left(\vec{u}_{1}+\vec{u}_{2}\right) / 2$, of $\vec{u}_{1}$ and $\vec{u}_{2}$.

Using the single-particle transition probabilities, $\pi_{1+2}(\vec{v})$ and $\tilde{\pi}_{1+2}(\vec{v})$, and the anologue ones for nucleus $2, \pi_{2 \rightarrow 1}(\vec{v})$ and $\tilde{\Pi}_{2+1}(\vec{v})$, we are ready to calculate all transport coefficients for the particle-exchange model.

The probability that nucleus 2 will change 1 ts momentum by an amount $\vec{n}$ during time $\tau$ can be expressed as

$$
W_{2}(\vec{n})=\int_{\text {window }} \frac{d^{2} v}{\Delta v} \int d^{3} v\left\{\pi_{1+2}(\vec{v}) \delta(\vec{n}-m \vec{v})+\tilde{\Pi}_{1+2}(\vec{v}) \delta(\vec{n}+m \vec{v})\right\} \cdot(3.5 .7)
$$

From all poss ble transitions we take only those with velocities which lead to the desired momentum change $\vec{\eta}$. The holes contrihute if miv=- $\vec{\eta}$. In addition, we sum over the whole window area. Correspondingly, the probability for nucleus 1 to change its momentum by $\vec{n}$ is given by 


$$
W_{1}(\vec{n})=\int_{\text {window }} \frac{d^{2} v}{\Delta v} \int d^{3} v\left\{\pi_{2+1}(\vec{v}) \delta(\stackrel{+}{n}-m \vec{v})+\tilde{\pi}_{2+1}(\stackrel{+}{v}) \delta(\stackrel{+}{n}+m \overrightarrow{+})\right\} .
$$

Conservation of the total momentum requires that a change $\vec{n}$ in the momentum of nucleus 2 is correlated with a change $-\vec{n}$ in the momentum of nucleus 1 and hence with a change $\vec{n}$ in the relative momentum $\overrightarrow{\mathrm{p}}=\overrightarrow{\mathrm{p}}_{2}=-\overrightarrow{\mathrm{p}}_{1}$. This many-body correlation is of course not observable in the single-particle phase-space distribution. Therefore, $w_{2}(\vec{n})$ and $w_{1}(-\vec{n})$ are in general not exactly equal. They coincide only when the two nuclei have equal temperatures and Fermivelocities. For that reason we take the averaged value of $w_{1}(-\vec{n})$ and $w_{2}(\vec{n})$ for the conditional probability $w(\vec{p}, \vec{q} \mid+\vec{n}, \tau)$ :

$$
w(\vec{p}, \vec{q} \mid \vec{n}, \tau)=\frac{1}{2}\left(W_{2}(\vec{n})+W_{1}(-\vec{n})\right) \simeq W_{2}(\vec{n}) \simeq W_{1}(-\vec{n}) .
$$

Thus, the explicit expression for the conditional probability characterizing the fiuctuating force between the nuclei is given by

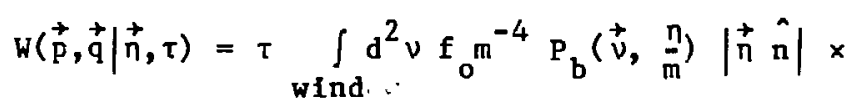

$$
\begin{aligned}
& \frac{1}{2}\left[g_{1}\left(\frac{\vec{n}}{m}\right)\left(1-g_{2}\left(\frac{\vec{n}}{m}\right)\right)+g_{2}\left(-\frac{\vec{n}}{m}\right)\left(1-g_{1}\left(-\frac{\vec{n}}{m}\right)\right)\right\} \text {. }
\end{aligned}
$$

The r.h.s. depends on the macroscopic variables $\vec{p}$ and $\vec{q}$ via the two Fermidistributions $g_{1}(\vec{v})$ and $g_{2}(\vec{v})$ for the gases in the vicinity of the window. One should note that the velocity mismatch, $\Delta \vec{u}$, at the window is the relative velocity of the two nuclei, $\vec{p} / \mu$, plus the mismatch of the intrinsic rotational velocity-fields. The geometry of the window is a function of the shape degrees (see subsection 3.1). Furthermore, $w(\vec{p}, \vec{q} \mid \vec{n}, \tau)$ depends on the two temperatures $T_{1}$ and $T_{2}$. 
At this point, let us summarize briefly the ideas behind eq. (3.5.10). Firstly, we make assumptions about the internal motion: The intrinsic degrees of freedom may be described by two Fermi-gases which serve as the so called heat bath. Each of them is characterized b a temperature, a chemical potential, a mean velocity and a rotational frequency. This is the least amount of information required for a rotating moving Fermi-gas. At the window the two gases are in contact and the exchange of part ales tends to equilibrate the four intensive macroscopic quantities. The Intrinsic time evolution in the vicinity of the window is assumed to be that of a long meanfree-path gas following the geometry of the window between the nuclei. Secondly, -n equilibration hypothesis enters which states that the distortion of the single-particle phase-space distribution initiated by the exchanged particles (see fig. 3.5 .1 ) equilibrates fast enough within one nucleus so that after a time $\tau$ there is no memory left which would influence the presumed single particle motion at the window. This means that particles which have crossed the window adopt the velocity distribution of the recipient nucleus before returning to the window. The equilibration can be thought of as collisions with the moving wall or residual two-body interactions. This Markovian behaviour might be questioned in the early stage of the collision [Nö81, Nö83]. Thirdly, the coupling between the intrinsic motion and the macroscopic degrees of freedom originates from the simple fact that the mean momentum of one nucleus is the sum over all intrinsic momenta. Taken together we obtain then the information, $w(\vec{p}, \vec{q} \mid \vec{n}, \tau)$, needed to describe the properties of the fluctuating force contained in the Langevin equation.

As derived in subsection 1.1 the components of the friction force are given by $F_{1}(\vec{p}, \vec{q})=\frac{1}{\tau} \int d^{3} \eta W(\vec{p}, \vec{q} \mid \vec{n}, \tau) \eta_{i}$ 


$$
\begin{aligned}
& =\int_{\text {window }} \mathrm{d}^{2} v \int \mathrm{d}^{3} v \mathrm{f}_{0} \mathrm{~m} \mathrm{P}_{\mathrm{b}}(\vec{v}, \vec{v})|\vec{v} \hat{\mathrm{n}}| \times \\
& \frac{1}{2}\left[g_{1}(\vec{v})\left(1-g_{2}(\vec{v})\right)+g_{2}(-\vec{v})\left(1-g_{1}(-\vec{v})\right)\right\} v_{1} .
\end{aligned}
$$

The components of the diffusion tensor are the second moments:

$$
\begin{aligned}
D_{1 j}(\vec{p}, \vec{q}) & =\frac{1}{2} \frac{1}{\tau} \int d^{3} n w(\vec{p}, \vec{q} \mid \vec{n}, \tau) n_{1} n_{j} \\
& =\int_{\text {window }} d^{2} v \int d^{3} v f_{0^{m}} P_{b}(\vec{v}, \vec{v})|\vec{v} \hat{n}| \times \\
& \frac{1}{2}\left\{g_{1}(\vec{v})\left(1-g_{2}(\vec{v})\right)+g_{2}(-\vec{v})\left(1-g_{1}(-\vec{v})\right)\right\} v_{1} v_{j} .
\end{aligned}
$$

Let us discuss some general features before turning to approximate evaluations of eqs. (3.5.11) and (3.5.12). The part in the curly brackets, which determines the phase space avallable for transitions, will govern the whole Integral. In flg. 3.5 .2 we illustrate in veluctty space those areas where the curly-bracket part is not vanishing. During the course of a nucleus-nucleus collision we have inftially a system with two sharp-edged Fermi spheres displaced by a velocity mismatch, $\Delta \vec{u}$, which is in this case the relative velocity (left hand part of fig. 3.5.2). Although the system is cold ( $\mathrm{T}=0$ ) we encounter already a large part of accessible phase space which gives rise to a non-vanishing diffusion coefficlent. Furthermore, one can guess from this picture that for zero temperature the magnitude of the diffusion tensor will be proportional to the absolute value of the velocity mismatch which is the thickness of the outer sickle-shaped parts. For the same reasons, the strength of the friction force ts proportional to the velocity mismatch. At a 
later stage the velocity has slowed down, the Fermi shperes overlap more and the excitation energy has smeared out the edges of the distribution so that additional transitions can take place (centre part of fig. 3.5.2). At a very advanced time during the collision the relative momentum and the angular momentum might have been dissipated completely such that transitions can occur only due to the fact that the system has a temperature (right hand part of fig. 3.5.2). It is only at this stage where we expect eqs. (3.5.11) and (3.5.12) to relate to each other via the Einsteln relation.

It is worthwhile to estimate the strength of the diffusion tensor if there is no temperature and only a shift in the velocity distributions zelative to the case where $\Delta \vec{u}=0$ and $T \neq 0$. A typical velocity mismatch for 1 MeV per particle is $1.410^{-22} \mathrm{fm} \mathrm{s}^{-1}$. On the other hand the smearing of the

velocity space
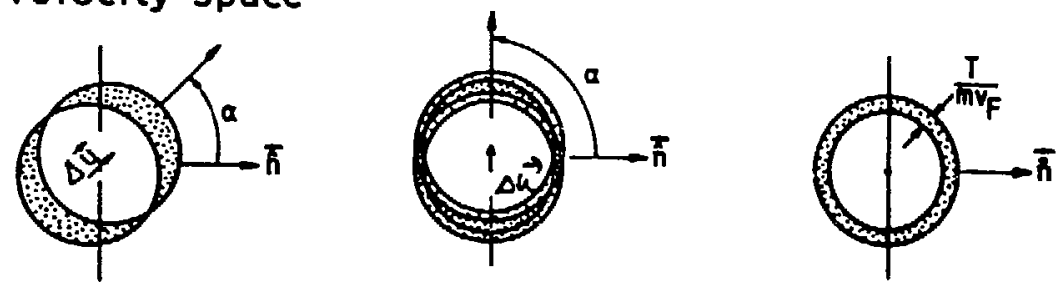

collision time

Fig. 3.5.2 Illustration of the part in the velocity space which contributes to particle transfer (dotted areas). The left hand part shows the case in which only the velocity mismatch $|\Delta u|+$ opens phase space and $T=0$. The center part for an intermediate situation and the right hand part for thermal limit where the temperature determines the avallable phese space and $\Delta u=0$. 
Fermi edge for typically $\mathrm{T}=2 \mathrm{MeV}$ is given by $\Delta \mathrm{v}=\mathrm{T} / \mathrm{mv} F=0.2410_{\mathrm{F}}^{-22} \mathrm{fm} \mathrm{s}^{-1}$. These two velocities correspond to the shift of the distributions and the broadening of the Fermi edge in fig. 3.5.2, respectively. This tells us already that the effect originating from the velocity mismatch will be important and cannot be neglected.

The expression (3.5.11) also allows for a clarifying remark on the Pauli-blocking effects in the window-friction force. If we would have forgotten the blocking factors $\left(1-g_{2}(\vec{v})\right)$ and $\left(1-g_{1}(-\vec{v})\right)$ in the curly brackets of eq. (3.5.11) the result for the friction force would not change at all. The reason is that the forgotten part:

$$
\int \mathrm{d}^{3} \mathrm{v} \mathrm{P}_{\mathrm{b}}(\vec{v}, \overrightarrow{\mathrm{v}})|\vec{v} \hat{\mathrm{n}}| \frac{1}{2}\left\{\mathrm{~g}_{1}(\vec{v}) g_{2}(\vec{v})+g_{2}(-\vec{v}) g_{1}(-\stackrel{+}{v})\right\} v_{1}=0
$$

vanishes because the Integrand is antisymetric with respect to the transformation $\vec{v}+-\vec{v}$. Here, we presumed that the penetrability, $P_{b}$, is symmetric in $\vec{v}$. A deeper reason for this formal argument lies in the one-body nature of the friction force, namely, one-body quantities are not affected by the Pauli-principle. To emphasize this point once again, the Pauli blocking has no effect on the window friction force. However, it is important when calculating two-body quantities like the diffusion tensor. In eq. (3.5.12) the above argument does not hold any longer since the corresponding part is now symmetric with respect to $\vec{v}+-\vec{v}$.

Another example is the diffusion in mass number. The mass diffusion coefficient is given by

$$
D_{A A}=\frac{1}{2} \frac{1}{\tau} \underset{k}{\sum} W\left(A_{2} \mid k, \tau\right) k^{2},
$$


where $W\left(A_{2} \mid k, \tau\right)$ is the conditional probability that the mass of nucleus 2 will change by $k$ unt ts during time $\tau$. As was the case for the relative momentum, it con be calculated with the help of $\pi_{1+2}\left(\stackrel{\vec{v}}{)}, \tilde{\pi}_{1+2}(\vec{v}), \Pi_{2+1}\left(\stackrel{\vec{v}}{)}\right.\right.$ and $\tilde{\Pi}_{2 \rightarrow 1}(\vec{v})$.

$$
\begin{aligned}
& W\left(A_{2} \mid 1, \tau\right)=\int_{\text {window }} \frac{d^{2} v}{\Delta v} \int d^{3} v \frac{1}{2}\left\{\pi_{1+2}(\vec{v})+\tilde{\pi}_{2 \rightarrow 1}(\vec{v})\right\} \\
& W\left(A_{2} \mid-1, \tau\right)=\int_{w i n d o w} \frac{d^{2} v}{\Delta v} \int d^{3} v \frac{1}{2}\left[\tilde{\pi}_{1+2}(\vec{v})+\Pi_{2+1}(\vec{v})\right\} \\
& W\left(A_{2} \mid k, \tau\right)=O\left(\tau^{2}\right) \text { for }|k|>1 .
\end{aligned}
$$

Thus, one arrives at

$D_{A A}(\vec{p}, \vec{q})=$

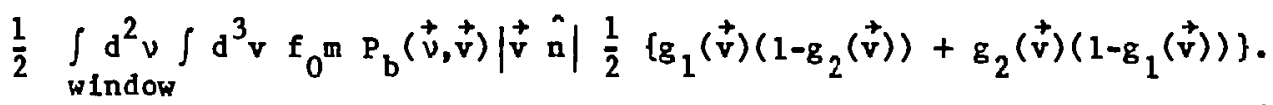

Next we will derive approximate solutions for the integrals appearing in the friction force (eq. (3.5.11)) and the diffusion tensor (eqs. $(3.5 .12)$ and $(3.5 .16))$ under the assumption that the velocity mismatch, $\overrightarrow{\Delta \vec{u}=\vec{u}_{2}}-\vec{u}_{1}$, is small compared to the Fermi velocity, $v_{F}$, and the two temperatures are almost the same and small compared to the Fermi energy. Let us denote with $b\left(\vec{v}, \Delta \vec{u}, T_{1}, T_{2}\right)$ the part in the curly brackets appearing in eqs. $(3.5 .11)$ and $(3.5 .12)$ :

$$
\mathrm{b}\left(\stackrel{\vec{v}}{\mathrm{v}}, \Delta \overrightarrow{\mathrm{u}}, \mathrm{T}_{1}, \mathrm{~T}_{2}\right) \equiv \frac{1}{2}\left\{\mathrm{~g}_{1}(\stackrel{\vec{v}}{\mathrm{v}})\left(1-\mathrm{g}_{2}(\overrightarrow{\mathrm{v}})\right)+\mathrm{g}_{2}(-\overrightarrow{\mathrm{v}})\left(1-\mathrm{g}_{1}(-\overrightarrow{\mathrm{v}})\right)\right\}
$$


Since expression (3.5.17) is symmetric under permutation of $\mathrm{T}_{1}$ and $\mathrm{T}_{2}$ $\mathrm{b}\left(\overrightarrow{\mathrm{v}}, \Delta \overrightarrow{\mathrm{u}}, \mathrm{r}_{1}, \mathrm{~T}_{2}\right)=\mathrm{b}(\overrightarrow{\mathrm{v}}, \Delta \overrightarrow{\mathrm{u}}, \mathrm{T}, \mathrm{T})+\mathrm{O}\left(\left(\mathrm{T}_{2}-\mathrm{T}_{1}\right)^{2}\right)$, where $\mathrm{T}=\left(\mathrm{T}_{1}+\mathrm{T}_{2}\right) / 2$. Assuming a small temperature difference between the nuclei, we evaluate eq. (3.5.17) for the mean temperature, $T$, only. In \& velocity frame where $\vec{v}_{1}=-\vec{u} / 2$ and $\vec{u}_{2}=\vec{u}^{\prime} / 2$, eq. $(3.5 .17)$ can be written as:

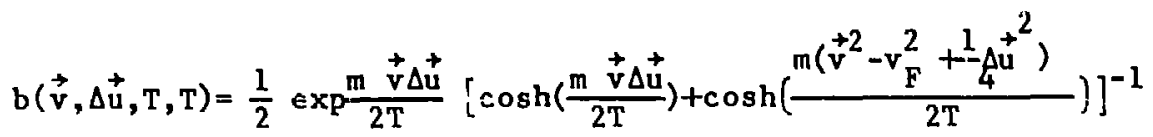

If $|\Delta \vec{u}| \ll<v_{F}$ and $2 T<<m v_{F}^{2}$ one gets contributions only from near the Fermi-edge. Therefore, one can approximate $\mathrm{b}(\overrightarrow{\mathrm{v}}, \Delta \overrightarrow{\mathrm{u}}, \mathrm{T}, \mathrm{T})$ by:

$$
\begin{aligned}
& \mathrm{b}(\overrightarrow{\mathrm{v}}, \Delta \overrightarrow{\mathrm{u}}, \mathrm{T}, \mathrm{T})=\frac{\mathrm{m \vec {v }} \Delta \overrightarrow{\mathrm{u}}}{\exp (\mathrm{m} \overrightarrow{\mathrm{v}} \Delta \overrightarrow{\mathrm{u}} / \mathrm{T})} \frac{\delta\left(\mathrm{v}-\mathrm{v}_{F}\right)}{\mathrm{mv}_{F}} \\
& =\left\{\mathrm{m}|\vec{v} \Delta \vec{u}| \theta(-\vec{v} \Delta \vec{u})+T \frac{m|\vec{v} \Delta \vec{u}| / T}{\exp (m|\vec{v} \Delta \vec{u}| / T)-1}\right\} \frac{\delta\left(v-v_{F}\right)}{{ }_{F} v_{F}}
\end{aligned}
$$

Thus, we see from the curly-bracket part, $b$, that the friction force as well as the diffusion tensor decomposes into two parts. The first - called the velocity part - is proportional to the velocity mismatch, $\Delta \vec{u}$, whereas the second - called the temperature part - is proportional to the mean temperature, $T$, of the system. The step function, $\theta$, as well as the function accompanying $T$, ranges between zero and one. Therfore, a measure of the relative strength of the two contributions is given by the ratio $\omega=\mathrm{mv}_{F}|\Delta \vec{u}| / T$. Discussions made earlier in this subsection show that nel ther of the two terms can be neglected. In the early stage of the collision and during all time for peripheral reactions the velocity part will dominate since $\omega>1$. At a late stage the velocity mismatch at the window disappears $(w=0)$ and one has $b=T \delta\left(v-v_{F}\right) / m v_{F}$, which 1 s the case where the 
Einstein relation holds. In section 3.7 we will show $w$ as a function of time as it results from trajectory calculations.

Calculating the friction force according to eq. (3.5.11) one realizes Immediately that the temperature part does not contribute since it is symmetric with respect to the tranformation $\vec{v}+-\vec{v}$. Thus, the window-friction force is in this afproximation temperature independent. In section 3.4 .2 we were more elaborate in this respect and worked out the temperature dependence of the fiction coefficients. As shown in fig. (3.4.7), it depends only weakly on the temperature. Here we obtain

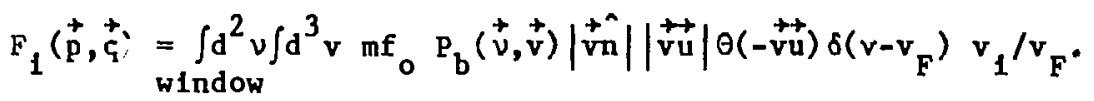

One possibility to avold the problem of finding an appropriate expression for the penetrability of the single-particle barrier at the window is to approximate the integration over the window by

$$
\int d^{2} v \int d^{3} v P_{b}(\stackrel{\vec{v}}{v}, \vec{v}) \ldots .=\pi v_{\text {eff }}^{2}(\stackrel{+}{q}) \int d^{3} v \ldots \ldots
$$

where the effective window size, $\pi \nu_{\text {ef }}^{2}(\vec{q}), 18$ given by

$$
\pi v_{\text {eff }}^{2}(\stackrel{+}{q})=\pi v_{0}^{2}(\stackrel{+}{q})+v_{v_{\min }}^{v_{\max }} \Psi(D(+\vec{q}, v)) 2 \pi v d v
$$

Here, $\Psi(D)$ is the universal proximlty funciton for the one-sided flux as determined by J. Randrup [Ra78]:

$$
\Psi(D)=\left\{\begin{array}{cl}
\left(\cos \left(\frac{D}{3.2} \frac{\pi}{2}\right)\right)^{2} & \text { for } 0<D<3.2 \\
0 & \text { for } 3.2<D
\end{array}\right.
$$


The effective window size which depends on the shape, consists of the geometrical opening, $\pi v_{m i n}^{2}$, and a proximfty part. If the two nuclet are separated $\left(v_{m i n}=0\right)$ the proximity part contributes only. (The nomenclature Is the same in section 3.4.2). One could also go along the lines of subsection 3.4 .2 and calculate first friction and diffusion coefficients for a planar geometry and use the proximity Idea after that. Randrup followed this way [Ra78a, Ra79, Ra82] and worked out different universal functions for drift and diffusion.

By assuming the velocity mismatch to be uniform over the window area, the friction force is given by:

$$
F_{1}(\vec{p}, \vec{q})=\pi v_{\text {eff }}^{2}(\stackrel{\vec{q}}{\mathrm{q}}) \mathrm{mf}{ }_{0} \int \mathrm{d}^{3} \mathrm{v}|\stackrel{+}{\mathrm{v} n}||\overrightarrow{v u}| \theta(-\vec{v} u) \delta\left(v-v_{F}\right) v_{1} / v_{f}
$$

The evaluation of this integral results in the well known window formula [BB78] or In the formula derived in section 3.4 .2 for temperature $T=0$ :

$$
\begin{aligned}
& F_{1}(\stackrel{+}{p}, \vec{q})=-\pi v_{\text {eff }}^{2}(\vec{q}) \frac{3}{16} m \rho v_{F} \sum_{k=1}^{3}\left(d_{1 k}+\hat{n}_{1} \hat{n}_{k}\right) \Delta u_{k} \\
& =-\sum_{k=1}^{3} \gamma_{1 k}{ }_{k} u_{k}
\end{aligned}
$$

It consists of the effective window size multiplied by the one-sided flux and by a non-isotropic tensor acting on the velocity mismatch. This tensor is twice as large in the direction of the window-normal than in the perpendicular directions $\left(\hat{n}=\left(\hat{n}_{1}, \hat{n}_{2}, \hat{n}_{3}\right), \Delta \vec{u}=\left(\Delta u_{1}, \Delta u_{2}, \Delta u_{3},\right)\right)$. The spatial particle density is denoted by $p=3 /\left(4 \pi r_{0}^{3}\right)$. 
According to eq. $(3.5 .19)$ we decompose the diffusion tensor into two parts:

$$
D_{i j}=|\Delta \vec{u}| m v_{F} D_{1 j}^{u}(\alpha)+T D_{1 j}^{T}(\alpha, w)
$$

As anticipated earlier, we encounter a contribution proportional to the absolute value of the velocity mismatch, $\left|\Delta u^{+}\right|$, and one proportional to the temperature, T. The velocity part is given by

$$
D_{i j}^{u}(\alpha)=\frac{1}{2} \pi v_{e f f}^{2}(\stackrel{+}{q}) m f_{0} \int d^{3} v|+\hat{v n}||\overrightarrow{v u}| \theta(-\overrightarrow{v u}) \delta\left(v-v_{F}\right) v_{1} v_{j} / v_{F}^{2},
$$

with $\hat{u}=\overrightarrow{\Delta u} /|\Delta \vec{u}|$ and $\cos \alpha=\hat{u n}$. Invarfance under rotation requires that $v_{i j}^{u}(\alpha)$ depends only on the angle $\alpha$ between the window-normal $\vec{n}$ and the velocity unft-vector $\hat{u}$. After a somewhat lengthy calculation we arrive at the following expression for the tensor, $D_{i j}^{u}(\alpha)$, written in a coordinate independent manner:

$$
D_{1 j}^{u}(\alpha) m C(s) \frac{8}{15 \pi}\left\{\left(3 \alpha+c_{\alpha} k_{\alpha}\right) \delta_{1 j}+s_{\alpha} \hat{n}_{1} \hat{n}_{j}+s_{\alpha} \hat{u}_{1} \hat{u}_{j}+k_{\alpha}\left(\hat{u}_{1} \hat{n}_{j}+\hat{u}_{j} \hat{n}_{1}\right)\right\}
$$

where $c_{\alpha}=\hat{u n}=\cos \alpha, s_{\alpha}=\sin \alpha$ and $k_{\alpha}=\left(\frac{1}{2} \pi-\alpha\right)$ for $0<\alpha<\pi$ and the onesided current $C(\vec{q})=\pi v_{\text {eff }}^{2}\left(q^{+}\right) \frac{3}{16} \rho v_{F}$.

The temperature part given by

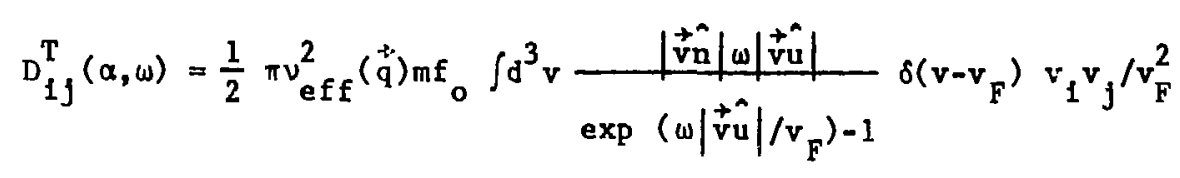


depends on $\alpha$ as well as on the ratio $\omega=|\Delta \vec{u}| m v_{F} / T$. In the 1 imit $\omega+0$, meaning no velocity mismatch but a finite temperature, the w-dependent factor under the integral becomes one and $D_{i j}$ does not depend on $\alpha$ anymore. For this case, it can be shown easily that an Einstein relation holds:

$$
D_{i j}=T D_{1 j}^{T}=T Y_{1 j} \text { for }|\Delta \vec{u}|=0
$$

However, as soon as $|\Delta \vec{u}| m v_{F}$ is not small compared to the temperature $T$ (see eq. (3.5.26) the Einstein relation is not valid any longer.

Since the evaluation of the integral in eq. (3.5.29) leads to functions which cannot be expressed analytically, we employ the following approximation:

$$
\frac{|x|}{\exp (|x|)-1}=(1-|x| / 6)^{3} \theta(6-|x|)
$$

These two functions differ at most by 0.03 which is certainly good enough for our purpose. With the help of eq. (3.5.31) we calculate the coefficients $A_{1}(\alpha, \omega)$ appearing in the general expression for the temperature part of the diffusion tensor:

$$
D_{i j(\alpha, w)}^{T}=m C(s)\left\{A_{1}(\alpha, \omega) \delta_{i j}+A_{2}(\alpha, w) \hat{n}_{i} \hat{n}_{j}+A_{3}(\alpha, \omega) \hat{u}_{i} \hat{u}_{j}+A_{4}(\alpha, w)\left(\hat{u}_{i} \hat{n}_{j}+\hat{n}_{i} \hat{u}_{j}\right)\right\} .
$$


For $0<\omega<6$ the $A_{i}(\alpha, \omega)$ are given by:

$$
\begin{aligned}
& A_{1}(\alpha, \omega)=1-\frac{8 \omega}{15 \pi}\left(s_{\alpha}+c_{\alpha} k_{\alpha}\right)+\frac{\omega^{2}}{72}\left(2-s_{\alpha}^{2}\right)-\frac{2 \omega^{3}}{2835 \pi}\left(3 s_{\alpha}-s_{\alpha}^{3}+3 c_{\alpha} k_{\alpha}\right), \\
& A_{2}(\alpha, \omega)=1-\frac{8 \omega}{15 \pi} s_{\alpha}+\frac{\omega^{2}}{72} s_{\alpha}^{2}-\frac{2 \omega^{3}}{2835 \pi} 2 s_{\alpha}^{3}, \\
& A_{3}(\alpha, \omega)=-\frac{8 \omega}{15 \pi} s_{\alpha}+\frac{\omega^{2}}{72} 2-\frac{2 \omega^{3}}{2835 \pi}\left(6 s_{\alpha}+6 c_{\alpha} k_{\alpha}\right) \\
& A_{4}(\alpha, \omega)=-\frac{8 \omega}{15 \pi} \quad k_{\alpha}+\frac{\omega^{2}}{72} 2 c_{\alpha}-\frac{2 \omega^{3}}{2835 \pi}\left(3 s_{\alpha} c_{\alpha}+3 k_{\alpha}\right),
\end{aligned}
$$

while for $\omega>6$ they are approximated by:

$$
\begin{aligned}
& A_{1}(\alpha, \omega)=A_{1}(\alpha, \omega=6)\left(\frac{6}{\omega} s_{\alpha}^{2}+\frac{36}{\omega^{2}} c_{\alpha}^{2}\right) \text { and } \\
& A_{i}(\alpha, \omega)=A_{1}(\alpha, \omega=6)\left(\frac{6}{\omega} s_{\alpha}^{2}+c_{\alpha}^{2}\right) \text { for } 1=2,3,4 .
\end{aligned}
$$

Here, $A_{1}(\alpha, \omega=6)$ on the r.h.s. has to be taken from eqs. (3.6.32a). As previously, $c_{\alpha}=\hat{u n}=\cos \alpha, s_{\alpha}=\sin \alpha$ and $k_{\alpha}=\left(\frac{1}{2} \pi-\alpha\right)$ for $0<\alpha<\pi$.

If $\omega>6$ the approxination (3.5.31) leads to complicated expressions since one has to split the integration at $\omega|\stackrel{+a}{*}|=6 v_{F}$ into two parts. On the other hand, the temperature part is for $\omega=|\Delta \vec{u}| \mathrm{mv}_{\mathrm{F}} / \mathrm{T}>6$ already sma $1 \mathrm{l}$ compared to the velocity part. Therefore, we content ourselves with an approximation which contains only the leading power in $\omega^{-1}$ for large $\omega$. For $\alpha=0$, which means $\stackrel{+*}{\mathrm{v}^{-}}=\stackrel{+\wedge}{\mathrm{v}}$, the coefficient $A_{1}(\alpha, \omega)$ is decreasing 1 ike $\omega^{-2}$, whereas for $\alpha=\frac{1}{2} \pi$ it is decaying only like $\omega^{-1}$. The other coefficents decay 1ike $\omega^{-1}$ for $\alpha=\frac{1}{2} \pi$ and assume a constant value for $\alpha=0$. The approximation (3.5.32b) takes care of this behaviour. 
A general feature of the particle-exchange pictures is that the friction tensor as well as the diffusion tensor is proportional to the onesided current of particles through the window $\left(c(\vec{q})=\pi v_{\text {eff }}^{2}(\vec{q}) 3 \rho v_{F} / 16\right)$. This quantity plays the role of a form factor which varies with the macroscopic degrees of freedom $\vec{q}$.

The matrix elements of the relative velocity pert depend strongly on the angle between the window normal (symmetry axis of the shape) and the veloctty mismatch. In the lower left corner of fig. 3.5 .3 we display $D_{i j}$ for the case of vanishing temperature where only $D_{1 j}^{u}$ survives. The diffusion tensor is calculated in a coordinate frame in which $\hat{u}=(\sin \alpha, 0, \cos \alpha)$ and $\hat{n}$ $=(0,0,1)$. One sees that for $\alpha=0$, which means head-on collisions, the fluctuations, $D_{33}^{u}(\alpha)$, in the direction of the relative velocity are three times as large as in the other directions. For peripheral

collisions $\left(\alpha=\frac{1}{2} \pi\right)$ the matrix elements become more equal. There is only one off-diagonal element, namely $D_{13}(\alpha)$, since in this coordinate frame $\hat{u}$ and $\hat{n}$ have no component in the 2 -direction.

In the other extreme where one has only the temperature part (upper left corner of $f 1 g .3 .5 .3)$ the matrix $D_{i j}^{T}$ is diagonal and the window-normal direction is twice as strong as in the other directions. 


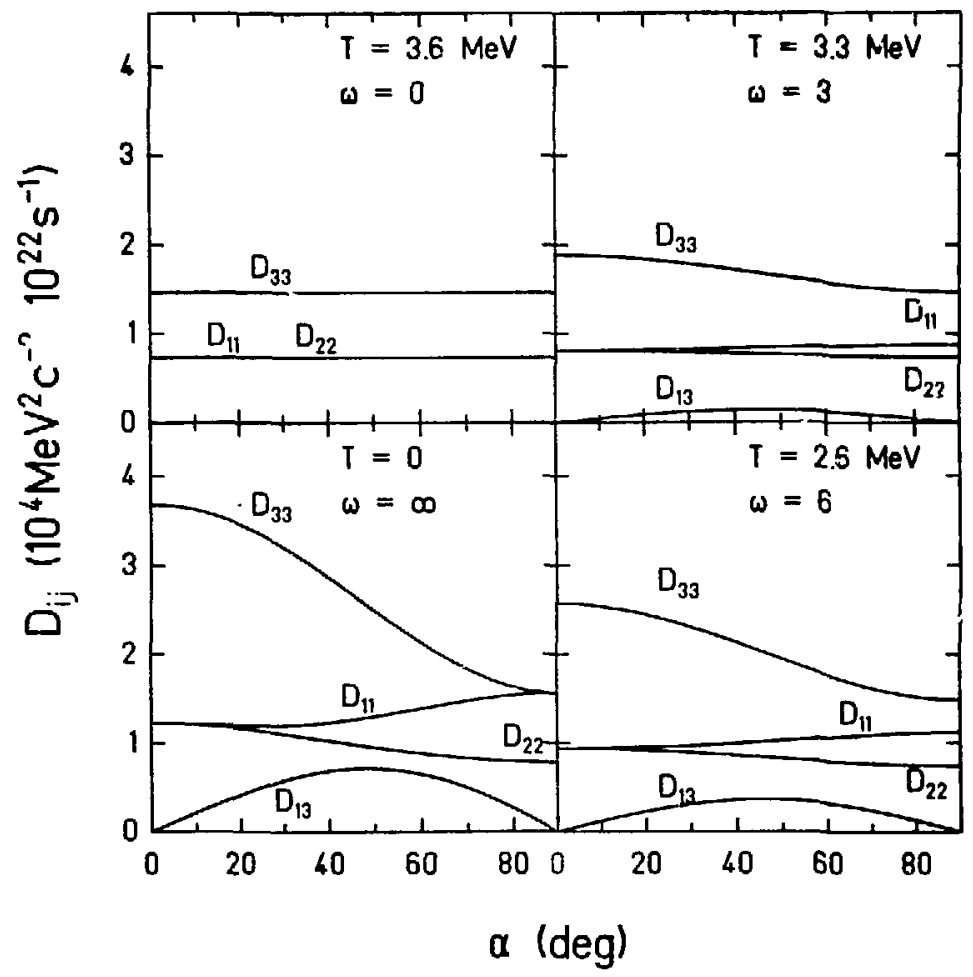

Figure 3.5.3 Maxtrix elements of the momentum diffusion tensor as a Eunction of the angle $\alpha$ between the window normal and the velocity mismatch. The coordinate frame is choosen such that the window normal has only a 3-component. The four different cases correspond to different values of the ratic $\omega=|\Delta \vec{u}| \mathrm{mv}_{F} / \mathrm{T}$. The scale corresponds to a typical window size. Please note the strong nonequilibrium diffusion for $T=0$ in the lower left corner.

If one chooses values for $\omega$ in beteen zero and infinity there will be a smooth transition between the two limiting cases. In the remaining part of fig. 3.5.3 the diffusion tensor is shown as it changes for different values of $\omega$ while keeping the combination $\left\{\left(\left.m ! \Delta \vec{u}\right|^{2} / 3+\pi^{2} T^{2} /\left(4 \varepsilon_{F}\right)\right\}\right.$ at a fixed value of $E_{0} / A$. this means that the system has a total c.m. energy per particle of $E_{0} / A$ which is shared between kinetic and heat energy according to $w$ with the kinetic energy being proportional to $|\Delta \vec{u}|^{2}$. In ectual trajectory calculations 
the relation between $|\Delta \vec{u}|$ and $T$ is more intricate. The two graphs on the right hand side of Fig. 3.5.3. Indicate that the Einstein like relation between diffusion and iriction is a good approximation for $\omega<3$. Let us call this the "temperature regime", the diffusion tensor does not decrease as we go wh the temperature to zero but it rather increases in size while changing the a-dependence. This is an important result since it affects strongly the calculated cross section. It will explain also why in nuclear collisions the mass drift can be hindered severely without diminishing the fluctuations in the mass number [FS84] (c.f. section 4.2).

The mass diffusion coefficient defined in eq. $(3.5 .16)$ can be easily calculated by taking the trace over $\mathrm{D}_{i j} /\left(\mathrm{mv}_{\mathrm{F}}\right)^{2}$ :

$$
D_{A A}=|\Delta \vec{u}| m v_{F} D_{A A}^{u}(\alpha)+T D_{A A}^{T}(\alpha, w),
$$

where the velocity part is given by

$$
D_{A A}^{u}(\alpha)=2 C(\vec{q}) / E_{F} \frac{2}{3 \pi}\left(s_{\alpha}+c_{\alpha \alpha} k\right)
$$

and for $0<\omega<6$ the temperature part is

$$
\begin{aligned}
& \mathrm{D}_{\mathrm{AA}}^{\mathrm{T}}(\alpha, \omega)=2 \mathrm{C}(\stackrel{+}{\mathrm{q}}) / \varepsilon_{\mathrm{F}} \text { * } \\
& \left\{1-\frac{2 \omega}{3 \pi}\left(s_{\alpha}+c_{\alpha} k_{\alpha}\right)+\frac{\omega^{2}}{48}\left(2-s_{\alpha}^{2}\right)-\frac{\omega^{3}}{810 \pi}\left(3 s_{\alpha}-s_{\alpha}^{3}+3 c_{\alpha} k_{\alpha}\right)\right\},
\end{aligned}
$$

whereas for $\omega>6$ we approximate 


$$
D_{A A}^{T}(\alpha, \omega)=D_{A A}^{T}(\alpha, w=6)\left(\frac{6}{\omega} s_{\bar{\omega}}^{2}+\frac{36}{\omega^{2}} c_{\alpha}^{2}\right)
$$

Here, the Fermi energy is denoted by $\varepsilon_{F}$ which is taken to be $32.11 \mathrm{MeV}$. The mean temperature $T$ is related to the heat energy $E^{*}$ residing in a Fermi gas with A nucleons by

$$
T=\frac{2}{\pi} \sqrt{ }\left(E^{\star} \varepsilon_{F} / A\right)
$$

Based on the same physical picture Randrup derived in a pioneer work [Ra78a, Ra79] the same diffusion tensor. However, his way of reasoning differs from ours in many aspects. Let us compare the final results by transforming Randrup's expression (Eq. (3.2) and eq. (2.23) of [Ra79]) into the present paper's nomenclature:

$$
D_{i j}^{\text {Randrup }}=\operatorname{mC}(s) \tau^{\star}\left\{\delta_{i j}+\hat{n}_{i} \hat{n}_{j}\right\}
$$

with

$$
\tau^{\star}=\frac{1}{2} \omega_{\text {eff }} \operatorname{coth}\left(\frac{1}{2} \omega_{\text {eff }} / T\right) \text { and } \omega_{\text {eff }}=\frac{1}{2}\left|\Delta u_{!}^{+}\right|_{F} d\left(1+(\cos \alpha)^{2}\right)
$$

First, one notes that for the temperature regime $\omega_{\text {eff }} \rightarrow 0$ and $\tau^{\star}+T$ his result coincides with ours but in the velocity regime $T+0$ and $\tau^{\star}+\frac{1}{4}|\Delta \vec{u}| \mathrm{mv}_{F} \sqrt{1+(\cos \alpha)^{2}}$ the tensor looks different from ours (c.f. eq. (3.5.18)). The dependence of the matrix elements on a differs strongly. However, the trace of the tensor which measures the overall strength of the fluctuations is only aboyt 10 percent large: than in our case 


$$
\sum_{1} D_{1 j}(\alpha, \omega+\infty) / \int_{1} D_{1 j}^{\text {Rand rup }}(\alpha, T=0) \simeq 0.85 \text { to } 0.94
$$

The reason for this is the approximation made in eq. (3.3) of [Ra79]). The same holds for the mass diffusion coefficient. 


\subsection{Equations of motion and conservation laws}

In this section we discuss the equations of motion for the model

discussed in the previous subsections. In addition we discuss the so called creeping 1 imit for the asymmetry and the neck degree of freedom. Unlike in section 1.1 where we derived quite generally the equations of motion we are going to use throughout this section lower case letters for the macroscopic variables. Nevertheless they are meant to be mean values of a narrowly peaked phase space distribution.

The equations of motion are

$$
\begin{aligned}
& \dot{q}_{i}=\left(M^{-1}\right)_{i}=\sum_{j}\left(M^{-1}\right)_{1 j} p_{j}, \\
& \dot{p}_{i}=-\frac{\partial T}{\partial q_{i}}-\frac{\partial V}{\partial q_{i}}-\sum_{j} R_{i j}\left(M^{-1}\right)_{j},
\end{aligned}
$$

where the indices run from 1 through 6 . The derivative of the kinetic energy $T$ is given by the derivative of the inverse mass tensor, or, if the velocities from eq. (3.6.1) are used, by the negative derivative of the mass tensor itself:

$$
\frac{\partial T}{\partial q_{k}}=\frac{1}{2} \sum_{i j} \frac{\partial\left(M^{-1}\right)_{1 j}}{\partial q_{k}} p_{i} p_{j}=-\frac{1}{2} \sum_{i j} \frac{\partial M_{i j}}{\partial q_{k}} \dot{q}_{i} \dot{q}_{j} \cdot
$$

The mass tensor $M$, the potential $V$, and the friction tenscr $R$ depend on $q$. They have been discussed in detail in subsections $3.2,3.3$, and 3.4 , respectively. In these equations the driving force which results from the temperature difference between the nuclei has not be included.

If the motion of the system is governed by strong frictional forces, the collision process can be divided into two stages. The system will first lose 
most of 1 ts kinetic energy residing in the radial motion ( $T_{\text {rad }}=1 / 2 \mu s^{2}$ ) during a short time of vollent deceleration. This is followed by a long creeping motion with little kinetic energy and practically no acceleration. The neck and the asymmetry degree of freedom do not possess inftial momentum. The kinetic energy residing in these two degrees of freedom bullds up during the collision. However, due to the strong frictional forces it turns out that the asymmetry, $q_{3}=\Delta$, is at all times overdamped. The neck degree of freedom, $q_{2}$, does contain kinetic energies up to about $2 \mathrm{MeV}$. If one neglects this, which has only little effect on the final results, one gets the following equations of inotion:

$$
\begin{aligned}
& \dot{q}_{1}=M_{11}^{-1} p_{i} \\
& \dot{q}_{1}=\left(\hat{R}^{-1} \hat{F}_{i} \text { for } i=2,3,\right. \\
& \dot{q}_{1}=M_{i 1}^{-1} p_{1} \text { for } i=4,5,6 \\
& \dot{p}_{1}=-\frac{\partial T}{\partial q_{1}}-\frac{\partial V}{\partial q_{1}}-\sum_{k=1}^{3} R_{1 k} \dot{q}_{k} \\
& \dot{p}_{2}=0 \\
& \dot{p}_{3}=0 \\
& \dot{p}_{i}=-\frac{\partial T}{\partial q_{1}}-\sum_{k=4}^{6} R_{i k} \dot{q}_{k} \text { for } i=4,5,6
\end{aligned}
$$

Here, we introduced the reduced friction tensor 


$$
\hat{R}=\left(\begin{array}{ll}
R & R \\
R_{32} & R_{33}^{23}
\end{array}\right)
$$

and the force $\hat{F}$ which has two components

$$
\hat{F}=\left(\begin{array}{c}
\hat{F}_{2}^{2} \\
\hat{F}_{3}
\end{array}\right)=\left(\begin{array}{l}
-\frac{\partial T}{\partial q_{2}}-\frac{\partial v}{\partial q_{2}}-R_{21} \dot{q}_{1} \\
-\frac{\partial T}{\partial q_{3}}-\frac{\partial v}{\partial q_{3}}-R_{31} \dot{q}_{1}+F_{\text {th }}
\end{array}\right) .
$$

The thermal force $F_{\text {th }}$ is given by

$$
F_{t h}=\frac{g_{A S}}{g_{A A}} \frac{d A_{1}}{d \Delta}\left(T_{2}-T_{1}\right) .
$$

The equation for $\dot{q}_{3} \equiv \dot{\Delta}$ corresponds to the first of eqs. $(3.4 .28)$ with $\Delta \mu$ replaced by all the different forces other than the thermal and the friction force. In addition we made use of the supposed one-to-one correspondence between $A_{1}$ and $\Delta\left(1\right.$. e. $\left.\dot{A}_{1}=\frac{d A_{1}}{d \Delta} \dot{\Delta}\right)$.

In all equations where a time derivative $\dot{q}$ appears on the r.h.s. It is meant to be replaced by the expression for $\dot{q}$ given in the preceding equations. The kinetic energy resides only in the degrees of freedom which are not overdamped. It is given by

$$
T=\frac{1}{2} M_{11}^{-1} p_{1}^{2}+\frac{1}{2} \sum_{1=4}^{6} M_{11}^{-1} p_{1}^{2}
$$

Besides the conservation of the total center of mass position and the total momentum the equations of motion conserve energy and angular momentum. The total conserved energy is given by 
$E_{\text {tot }}=T(p(t), q(t))+V(q(t))+\int_{0}^{t} d t^{\prime} \dot{q}\left(p\left(t^{\prime}\right), q\left(t^{\prime}\right)\right)-\int_{0}^{t} d t^{\prime} \dot{q}_{3} F_{t h}$

where

$$
\dot{Q}=\sum_{i, k=1}^{6} R_{i k} \dot{q}_{i} \dot{q}_{k}
$$

The last term in eq. (3.5.15) which contains the thermal force has to be omitted in the case of eqs. (3.5.1) - (3.5.3). The other constant of motion is the total anqular momentum given by

$$
L_{\text {tot }}=\sum_{1=4}^{6} M_{11} \dot{q}_{1}=I_{r e l} \dot{\theta}+I_{1} \dot{\theta}_{1}+I_{2} \dot{\theta}_{2}
$$

Both conservation laws can be proven by calculating the total time derivative $\frac{d}{d t}=\frac{\partial}{\partial t}+\sum_{i=1}^{6}\left\{\dot{q}_{i} \frac{\partial}{\partial q_{i}}+\dot{p}_{i} \frac{\partial}{\partial p_{i}}\right\}$ and using the equations of motion. The equations of motion for the second moments are given in general terms in section 1.1. A detafled application to all shape degrees of freedom will not be given here. In section 3.7 we discuss for peripheral collistons the time evolution of the second moments for the relative distance $s$ and the corresponding relative momentum while keeping $\sigma$ and $\Delta$ fixed at their initial values. Calculations by several authors [NS80, YN82] have shown that the assumption of narrowly peaked phase space distributions is not valid for deep inelastic reactions. There, the reaction time may be long enough to allow for a large spreading in coordinate space; the distribution covers such a wide range of the potential landscape that the harmonis. approximation fails (c.f. the discussion leading to eq. $(1.1 .26))$. 
For the mass asymmetry the equation of motion for the second moment assumes a rather simple form. This is due to the creeping assumption which neglects all inertial terms.

$$
\frac{d}{d t} \sigma_{A A}^{2}=2 \frac{d \Delta}{d A_{i}} \frac{\partial}{\partial \Delta}\left(E_{A A} \frac{d \Delta}{d A_{1}} \hat{F}_{3}\right) \cdot \sigma_{A A}^{2}+2 D_{A A}
$$

ite mobility coefficient, $g_{A A}$, has been calculated in section 3.4 .2 end $\hat{F}_{3}$ is defined in eq. $(3 \cdot 6 \cdot 12)$.

\subsection{Results of trajectory calculations}

In fig. 3.7.1 we display as a function of time different quantities along a trajectory. As an example for a deep inelastic collision we took a trajectory with an initial angular momentum $L=200 \mathrm{~K}$ for the case ${ }^{86} \mathrm{Kr}+{ }^{166} \mathrm{Er}$ with $E_{\mathrm{cm}}=465 \mathrm{MeV}$. In the upper part the total energy of the system is decomposed into potential energy $v$, plus kinetic energy of the shape, $T_{s}=\frac{1}{2} \sum_{i j=1}^{3} M_{1 j} \dot{q}_{i} \dot{q}_{j}$, plus kinetic energy residing in the relative rotation, $T_{r r}=\frac{1}{2} I_{r e l} \omega^{2}$, and in intrinsic rotation $T_{r 1}=\frac{1}{2} I_{1} w_{1}^{2}+\frac{1}{2} I_{2} w_{2}^{2}$. The remaining energy has been converted into heat, $Q=Q^{\text {window }}+Q^{\text {wall }}$. In the middle part of the figure the actual shape is plotted as it develops in time. At time t=0 the nuclear surfaces are $14 \mathrm{fm}$ apart. Within about $0.5^{*} 10^{-21} \mathrm{~s}$ they come into contact and, due to the proximity friction, the particle exchange has produced already about $15 \mathrm{MeV}$ of heat. After touching the radial motion is stopped rapidly. The turning point in $\mathrm{s}$ is reached at $t=0.8^{\cdot 10^{-21}} \mathrm{~s}$ and about $108 \mathrm{MeV}$ of kinetic energy has been dissipated. This short relaxation agrees rather well with estimates obtained from experimental data. Riedel, Wolschin and Nörenberg deduced the 
value $\tau_{\text {rad }}=(0.3-0.5) \cdot 1 \mathrm{C}^{-21} \mathrm{~s}[\mathrm{RW79}$, WE80]. On the other hand, this calls into question the randomization assumption, since this time is not long compared to the intrinsic equilibration time estimated by the time a nucleon needs to travel through the nucleus, $\tau_{\text {equ }}=2 \mathrm{R} / \mathrm{v}_{\mathrm{F}} \approx 0.2 \cdot 10^{-21} \mathrm{~s}$. The validity of a Markovian process is not beyond doubt for the approach phase. Now follows a long creeping motion of the shape degrees of freedom characterized by almost no kinetic energy in these degrees $\left(T_{s} m_{0}\right)$. During this period from $t=0.8 \cdot 10^{-21} \mathrm{~s}$ to $t \approx 4 \cdot 10^{-21} \mathrm{~s}$ deformations develop, as can be seen in the middle part of fig. 3.7.1. Again, this fits into the picture of Riedel et a1. [RW79], who supposed an over-damped motion for the deformations with a typical time $\tau_{\text {def }}=(1-5) \cdot 10^{-21} \mathrm{~s}[\mathrm{WE} 80]$. At $t=4.5 \cdot 10^{-21} \mathrm{~s}$ the shape has stretched to an elongated object with a thin neck. The nuclear system measures $34.7 \mathrm{fm}$ from tip to tip compared to two touching spheres with $2\left(R_{1}+R_{2}\right)=23.4 \mathrm{fm}$. About $20 \%$ of the matter 18 now in the neck reglon outside the spheres $(\sigma * 0,20)$. These large deformations allow the system to dissipate energy well below the Coulomb barrier of two spheres. However, not all of the deformation energy goes into heat. As can be seen from fig. 3.7.1, when the system comes close to the scission line, it again picks up kinetic energy. This is due to the rapid fall of the potential close to the scission Iine, which results in a strong force, accelerating the nuclei. In this way the system has gained about $33 \mathrm{MeV}$ of kinetic energy in the shape degrees of freedom when it scissions.

The heat produced by the dissipation is divided by the dotted ine into $Q^{\text {wall }}$ originating from the wall friction and $Q^{\text {window }}$ from the window friction. The wall dissipation contributes for about one-third to the total heat. Its major part is produced around $t=0.8 \cdot 10^{-21} \mathrm{~s}$ by the fast neck opening. 

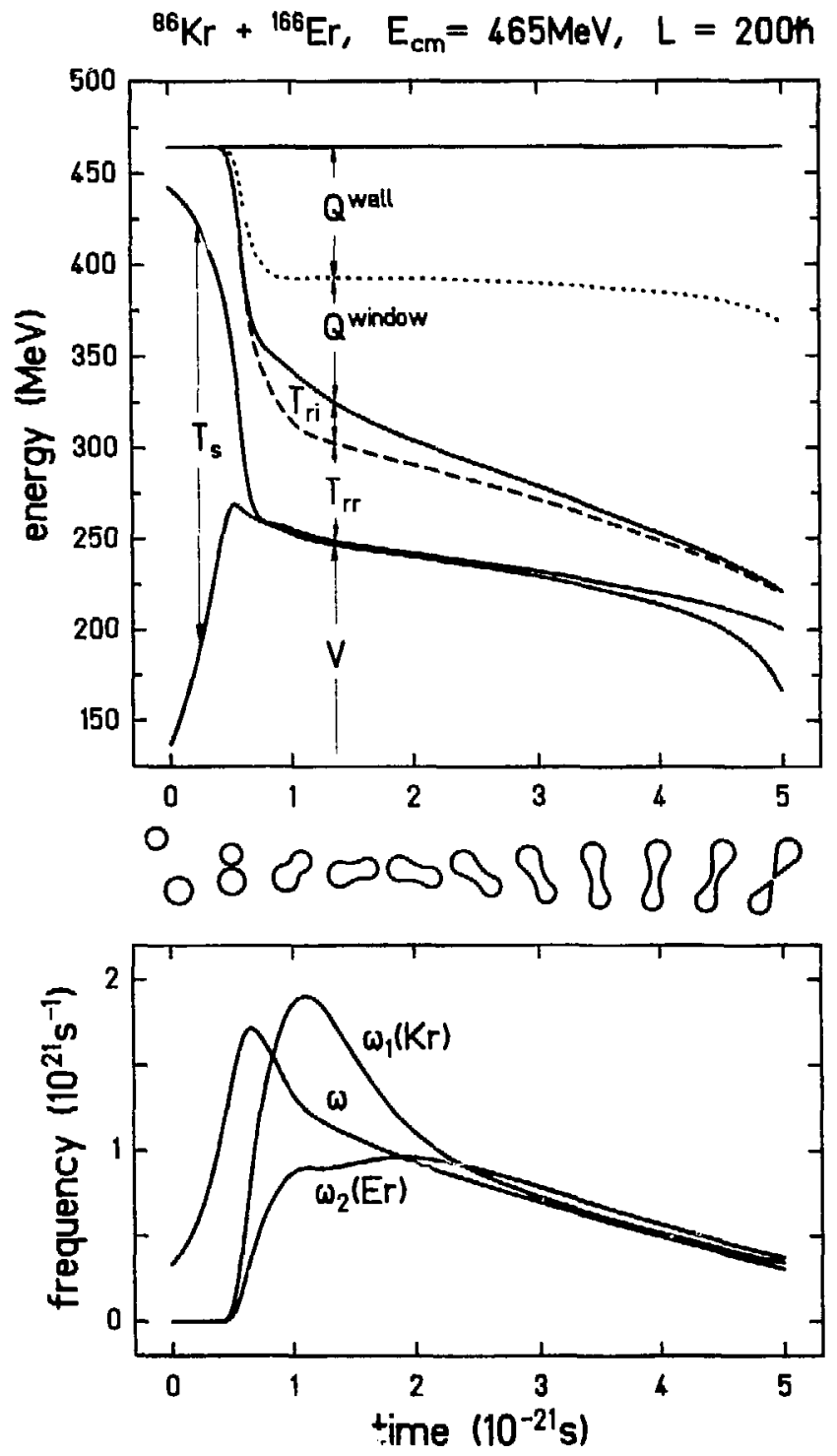

F1g. 3.7.1 Upper part: decomposition of the total energy Into heat produced by wall dissipation, $Q^{\text {wall }}$, window dissipation, $Q^{\text {window, }}$ rotational energy of the two 8 pheres, $T_{r 1}$, relative rotational energy, $T_{r r}$, kinetic energy in the shape of degrees of freedom, $T_{s}$, and potentlal energy, $V$. Centre part: shapes of the nuclear system. Lower part: angular frequencles. All quantitles are shown as a function of time during the collision of $86_{\mathrm{Kr}}$ and ${ }^{166} \mathrm{Er}$ for Inftial values of $\mathrm{E}_{\mathrm{cm}}=465 \mathrm{MeV}$ and $\mathrm{L}=200 \%$. 
The dissipation of the rotational kinetic energy is much slower than the relaxation in the radial degree of freedom. The lower part of fig. 3.7.1 shows that the angular frequencies need $1.810^{-21} \mathrm{~s}$ to become about equal. This number should be compared to the relaxation time $\tau_{1}=1.5 \cdot 10^{-21} \mathrm{~s}$ of Riedel et a1. [RW79]. The kinetic energy of the relative rotation, $T_{r r}$ is decreasing steadily up to sission. Finally, the two nuclei separate with an angul-r momentum of $175 \mathrm{~K}$ compared to $200 \mathrm{~K}$ they started with.

Tie longest relaxation time is seen in the equilibration of the mass asymmetry. The strong window friction hinders the system to reach a symmetric shape. In section 4.2 we will discuss this phenomenon, which leads to the deep inelastic heavy-ion collisions, in more detall. We want to emphasfze that without the window friction against the net particle flux through the window, the trajectories would proceed rather fast towards symmetry which for lighter systems would lead to fusion. The deef inelastic events would be suppressed.

In order to study the effects of the coupling between particle and entropy flux between the nuclei which has been discussed in section 3.4 .2 we use the more asymmetric system Ho $+F e$. The counteracting effects of the thermal force, $\left(g_{A S} / g_{A A}\right)\left(T_{2}-T_{1}\right)$, in comparison to the driving force, $\Delta \mu=\left(\hat{F}_{3}-F\right.$ th $) \frac{d \Delta}{\Delta A_{1}}$, is shown for three different trajectories in $\mathrm{fig}$. 3.7 .2 (c.f. eqs. $(3.6 .13),(3.4 .28)$ ). Please note that $-\Delta \mu$ has been plotted. At the beginning of the reaction the temperature difference $\Delta T=T_{2}-$ $\mathrm{T}_{1}$, builds up and the thermal force takes away a portion of the driving force. For the trajectory with an initial angular momentum of $L=220 \mathrm{~h}$ one sees toward the end of the reaction that the thermal feed back becomes rather substantial. Here, the system is for some time in a situation where the potential barrier between the two nuclei is close to the Fermi edge and the 
transport coefficients are very sensitive to the temperature. This happens around $s=14 \mathrm{fm}$ where $g_{A A} 1 s$ nuch smaller than $g_{A S}$ and therefore the ratio $g_{A S} / g_{A A}$ which enters the thermal force becomes large (see fig. 3.4.7). At the beginning of the reaction the two nuclei pass through this configuration in a short time so that the effect on the final mass number remains rather limited. For the case $\mathrm{L}=180 \mathrm{~h}$, a temperature difference exists during the whole collision time but the magnitude of the thermal force does not reach the values of the driving force. For $\mathrm{L}=140 \mathrm{~h}$ where the nuclei stick together for a long time the temperatures equilibrate and the feedback mechanism is active only during the first sixth of the reaction. Moretto [Mo83] has pointed out that the thermal feedback and self-regulation of the excitation energies could be a possible explanation why the drift in mass asymmetry is in many reactions so small and at the same time the sharing of the excitation energy seems to be proportional to the mass numbers. He performed his calculations taking the helght of the 3 ingle-particle potential-barrier as a time-independent parameter to illustrate the effect. In our calculation the barrier cannot be chosen but is dictated by the time evolution of the macroscopic degrees of freedom. The results show that the thermal feedback has no significant effect on the final values of the mass asymmetry for those cases we studied. In order to see the quantitative influence of thermal force on the final numbers we have set $g_{A S}=0$. The resulting change in the final charge numbers is so little that it could hardly be seen in fig. 3.7.2. Nevertheless, the mean charge number drifts very slowly. The main reason is the smallness of the mobility coefficient, $\mathrm{g}_{\mathrm{AA}}$, or since 1 ts inverse is the friction coefficient the very large window friction against mass drift. This effect will be discussed in greater detall in section 4.2 . 


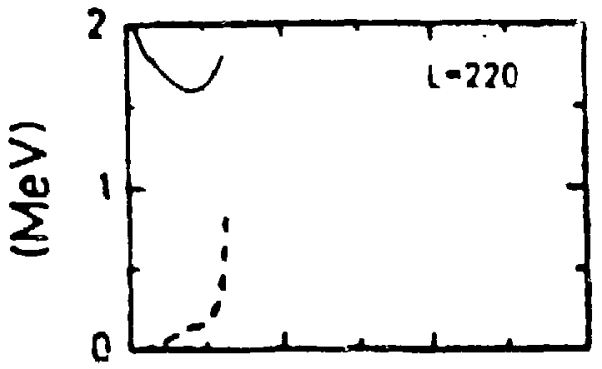

\author{
$\mathrm{Ho}+\mathrm{Fe}$ \\ $E_{\mathrm{cm}}=355 \mathrm{MeV}$
}
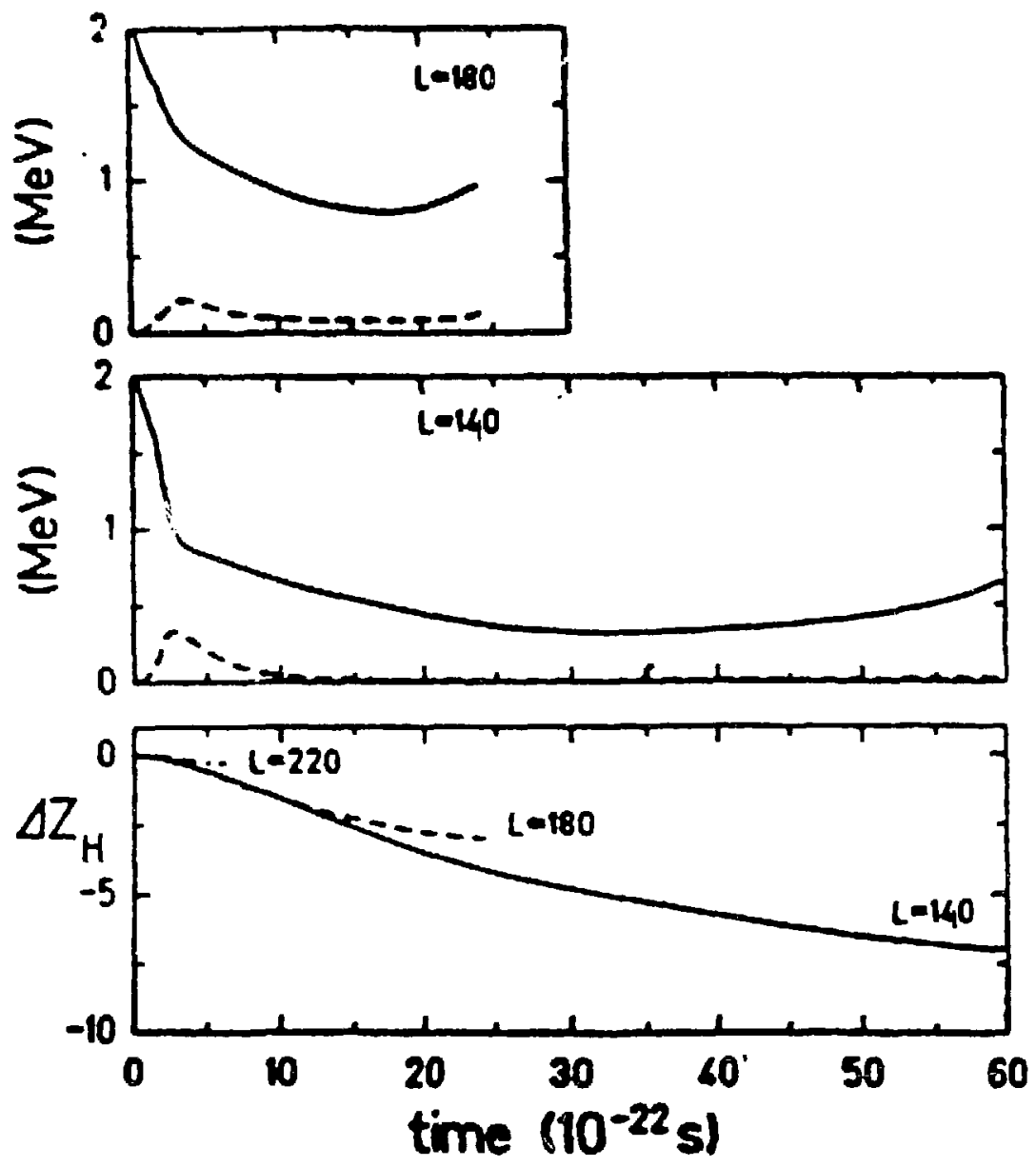

Fig. 3.7.2. Uppex three boxes: negative of the driving force (- $\Delta \mu: F u l 1$ lines) and thermal force (dashed lines) as a function of time for three L-values. Lower box: . change of the mean charge number as a function of time for the same trajectories. 
Let us discuss now the time evolution of the second moments which we obtain by solving eqs. (1.1.31)-(1.1.33). Again we consider the case ${ }^{86} \mathrm{Kr}$ on ${ }^{166} \mathrm{Er}$ with a beam energy of $8.18 \mathrm{MeV}$ per nucleon. However, in the following discussion we do not use all shape degrees of freedom but only the distance $s$ between the nuclei. The neck and the asymmetry degree of freedom are kept frozen at their intial values $\sigma=0$ and $\Delta=.11$. Therefore, we can study only collisions with a final kinetic energy above the Coulomb barrier where deformation effects are not so essential. The restricted family of shapes used is displayed in fig. 3.7.3. Nevertheless, this is an interesting regime because 1 it is here where we expect to see most prominently the nonequilibrium effects which give rise to fluctuations at zero or small temperatures.

In fig. 3.7 .4 we summarize the dependence of the energy loss, $E_{10 s s}=$ $E_{\mathrm{cm}^{-}} \mathrm{E}_{\mathrm{f}}$, the final deflection angle, $\theta_{\mathrm{f}}$, and the loss in relative angular momentum as a function of the Incoming angular momentum $L$. The curves start with $L=117 \not h$ because for lower $L=v a l u e s$ the systems gets trapped in the pocket of the potential. For a more detalled study we display for the Lvalues $280 \mathrm{~K}, 240 \mathrm{~K}, 200 \mathrm{~K}$ and $160 \mathrm{~h}$ several first and second moments as a function of time. In $\mathrm{fig}$. 3.7.5 the time dependence of the relative distance, $S=\left(x^{2}+z^{2}\right)^{1 / 2}$, of the relative velocity, $d S / d t$, and the form factor (effective window size $\pi v_{\text {eff }}^{2}$ ) is shown. For $L=280 k$ the nuclel do not touch and we have only dissipation due to the proximity effect. Correspondingly, the effective window size remains small and the radical velocity ds/dt is not slowed down too much. As soon as the nuclei touch and the geometrical window opens we encounter a delay in the reaction time and dS/dt stays almrat zero for some time $(L=200 h, 160 h)$. Trajectorles with L-values smaller than $160 \mathrm{~h}$ have rather long reaction times of more than $3 \cdot 1^{-21} \mathrm{~s}$ and one has to keep in mind that the system approaches fusion. 


\section{$s(f m)$}

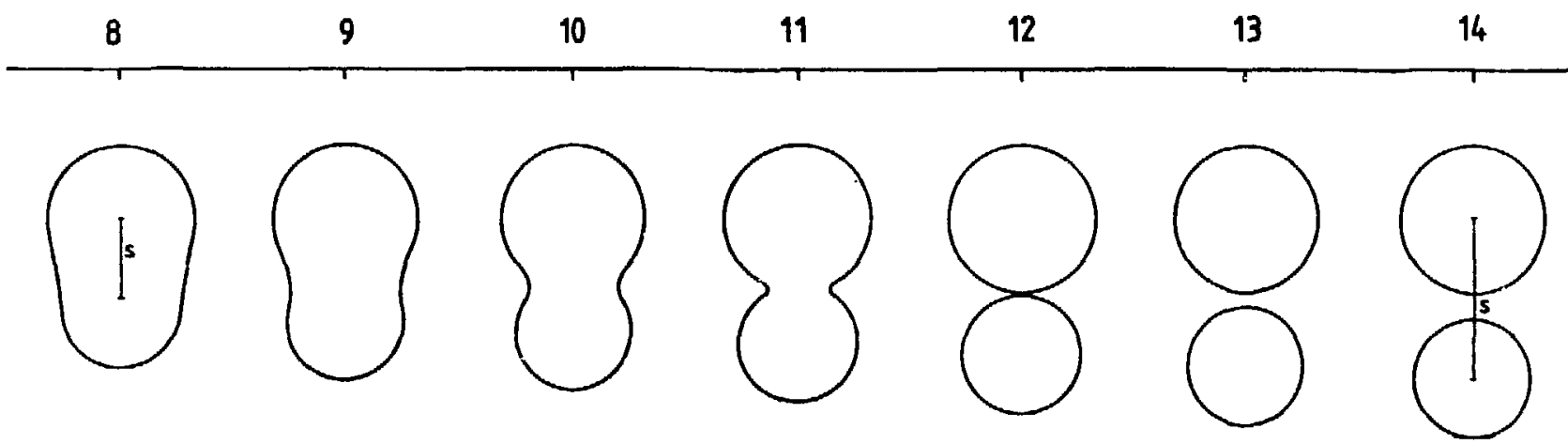

F15. 3.7.3 Restricted famlly of shapes ( $\sigma=0, \Delta=0.11$ ) used to calculate the second moments. 


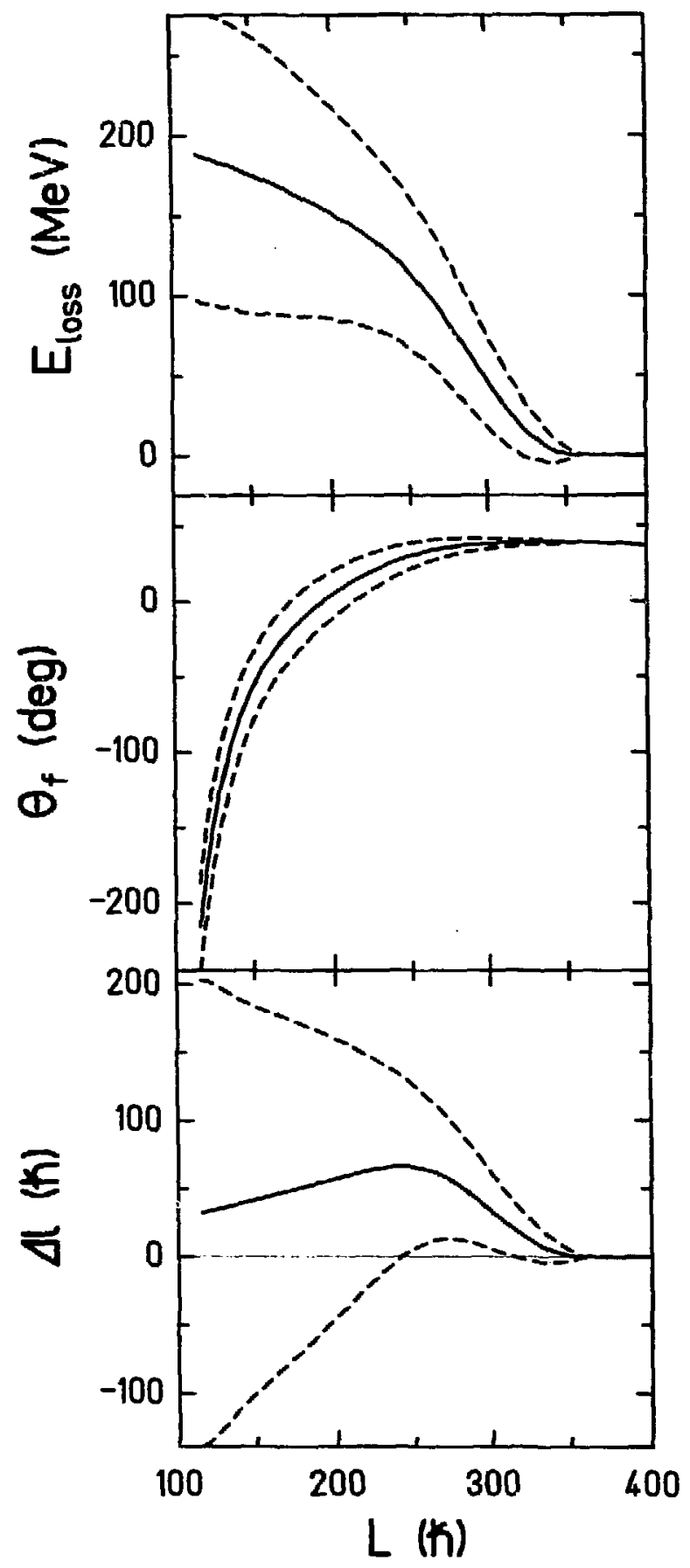

Fig 3.7.4 Energy 108s, finol ocattering angle and angular momentum 1088 as a function of the InItial L-value for ${ }^{86} \mathrm{Kr}+$ $166_{\mathrm{Er}}$ at $\mathrm{E}_{\mathrm{lap}}=8.18 \mathrm{MeV} / \mathrm{u}$ (solid lines). The differences between the dashed and the solld lines denote the corresponding final varlances $\sigma_{\mathrm{EE}}, \sigma_{\theta \theta}$ and $\sigma_{\mathrm{LL}}$. 


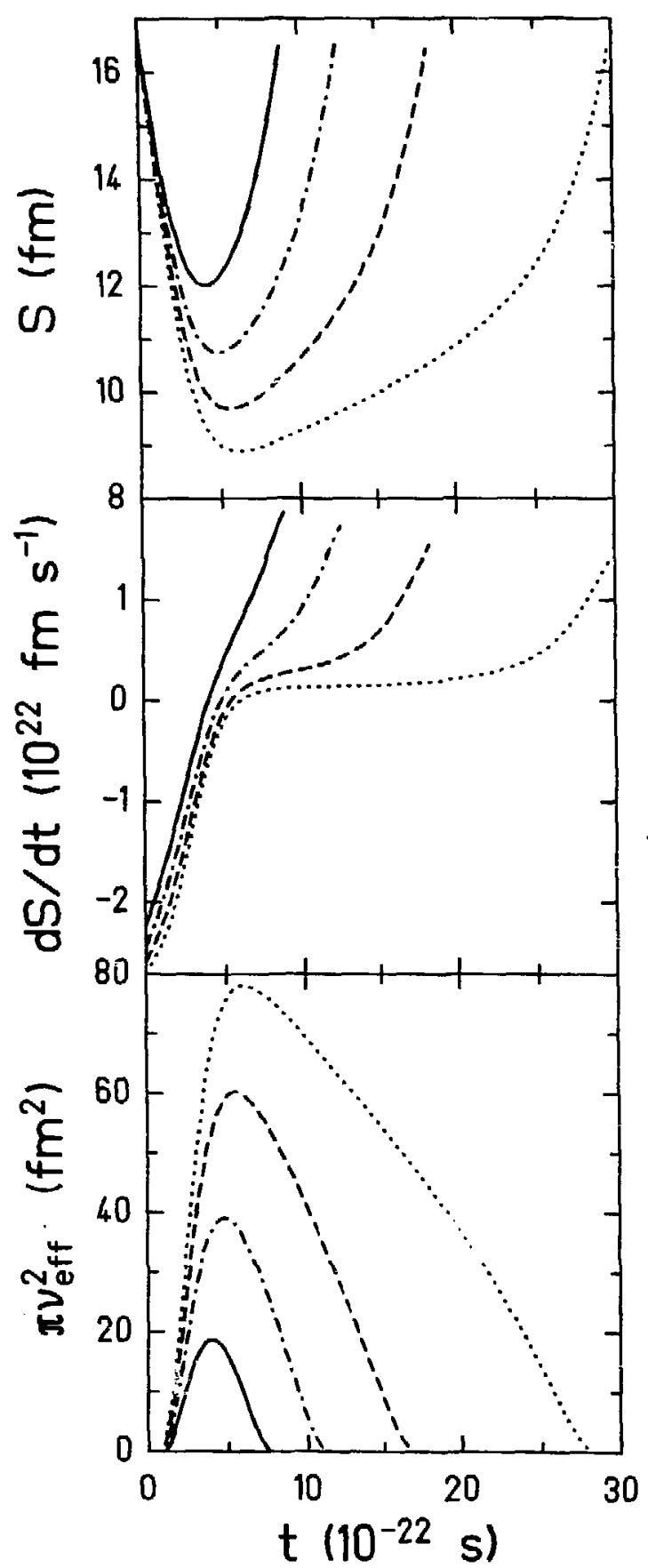

Fig.3.7.5 Relative distance, radial velocity and effective window area as a function of time for four trajectorles with the Initlal L-values: $\mathrm{L}=280 \mathrm{~K}--\mathrm{L}, \mathrm{L}=240 \mathrm{~K}-\ldots, \mathrm{L}=200 \mathrm{~K}-\cdots$, $\left.\mathrm{L}=160 \mathrm{~h} \ldots \mathrm{C}^{86} \mathrm{Kr}+{ }^{166} \mathrm{Er}, \mathrm{E}_{1 \mathrm{ab}}=8.18 \mathrm{MeV} / \mathrm{u}\right)$ 
These long reaction times bring up the question about the validity of approximations made concernig the small width and thereby the weak dependence of the forces on coordinates and momenta. To discuss these problems we display in $\mathrm{fig}$. 3.7 .6 the dependence of the conservative force, $\vec{k}$, and the friction force, $\vec{F}$, on the relative distance, $s$. In the upper part-dv/ds is shown while the lower part is a graph of the effective window size, $\pi v_{\text {eff }}^{2}$, which represents the essential dependence of the friction force on s. The Taylor expansion of the forces around the mean values $\vec{P}^{+}$and $\dot{Q}_{\text {(see }}$ eqs. (1.1.26) and $(1.1 .27)$ ) requires a phase-space distribution with a width not exceeding the range where the linear approximation is meaningful. Since $\vec{K}$ does not depend on $\vec{P}$, and $\vec{F}$ depends only linearly on $\vec{P}$ this approximation is always valid for $\vec{P}$. For the coordinates, however, one would estimate from the conservative force, $d V / d s$, that $\left(B_{x x}+B_{z z}\right)^{1 / 2}$, which is a measure for the width in the relative distance, should not exceed $1 \mathrm{fm}$. To check this we display in $\mathrm{fig} .3 .7 .7$ the time evolution of the according variances. One sees that for a 11 L-values above $160 \mathrm{~K}$ the width in coordinate space does not exceed $1 \mathrm{fm}$. These reactions correspond to times lese than $3 \cdot 10^{-21 s}$. For more central collisions the trajectories spend a rather long time around $S=9 \mathrm{fm}$ which is just the region where the conservative force is parabolic and hence approximated badly by the linerization (cf. fig 3.7.6). Here, we are already so close to fusion that a full solution of the Fokker-Planck equation would probably produce a distribution which splits into two parts, one which fuses and one which reseparates again [BR82]. This behaviour cannot be described in the moment expansion. In our calculations 
the variances become large, up to $4 \mathrm{fm}^{2}$ for $L=118 \mathrm{~h}$, but we do not observe "exploding" widths as encountered by other authors [NS80, YN82, CK81]. For completeness the momentum variances $A_{x x}, A_{z z}$ and $A_{x z}$ as well as $C_{x x}, C_{z z}, C_{x z}$ and $C_{z x}$ are displayed in fig. 3.7.8. In section 4.1 we will discuss those in a more conspicuous way by considering the fluctuations of the energy loss, scattering angle and angular momentum. The mixed variances $C_{x z}$ and $C_{z x}$ are almost equal which reflects the conservation law

$$
C_{a}=1_{1}+1_{2}+x p_{z}-z p_{x}-L_{\text {tot }}=0
$$

for the $y$-component of the total spin. Inserting this into eq. (1.2.7) we obtain

$$
C_{z x}-C_{x z} \approx 0
$$




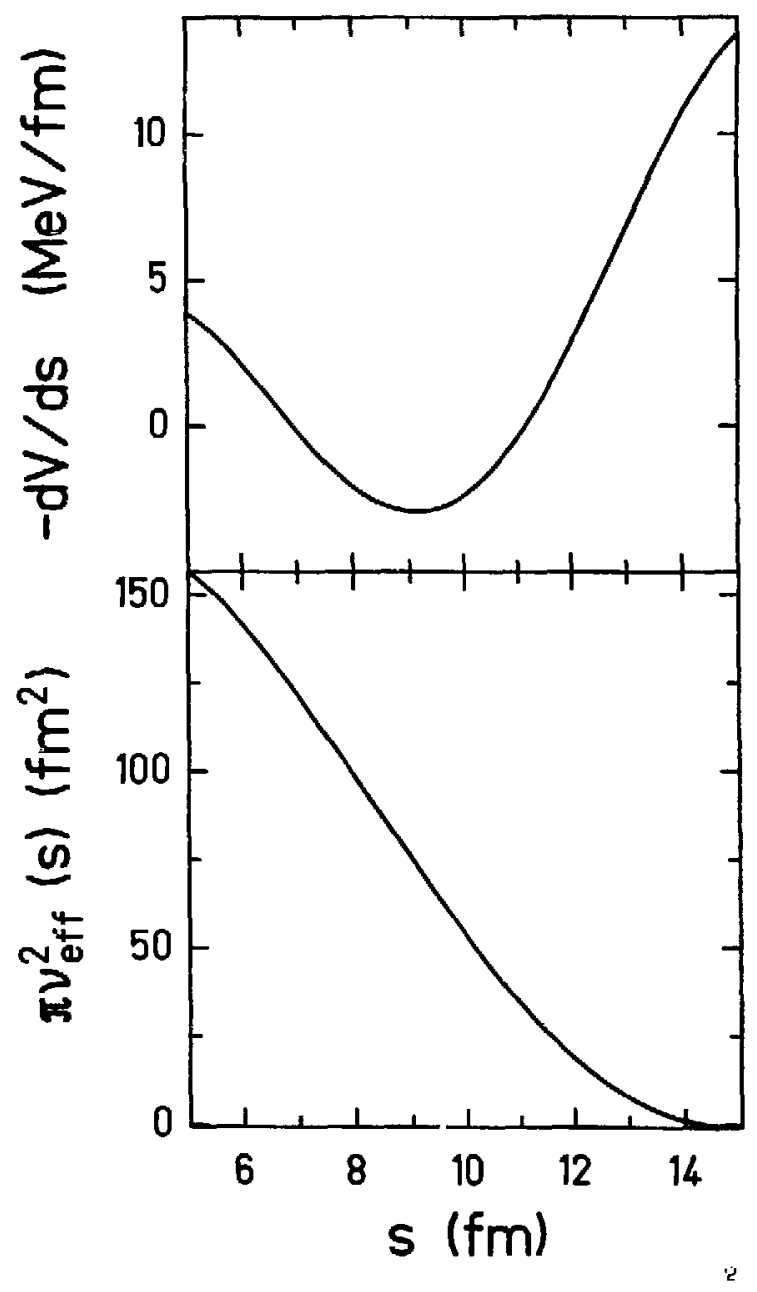

Fig. 3.7.6 Conservative force and effective window area as a function of the relative distance between the nucle? for ${ }^{86} \mathrm{Kr}+166_{\mathrm{Er}}$. 


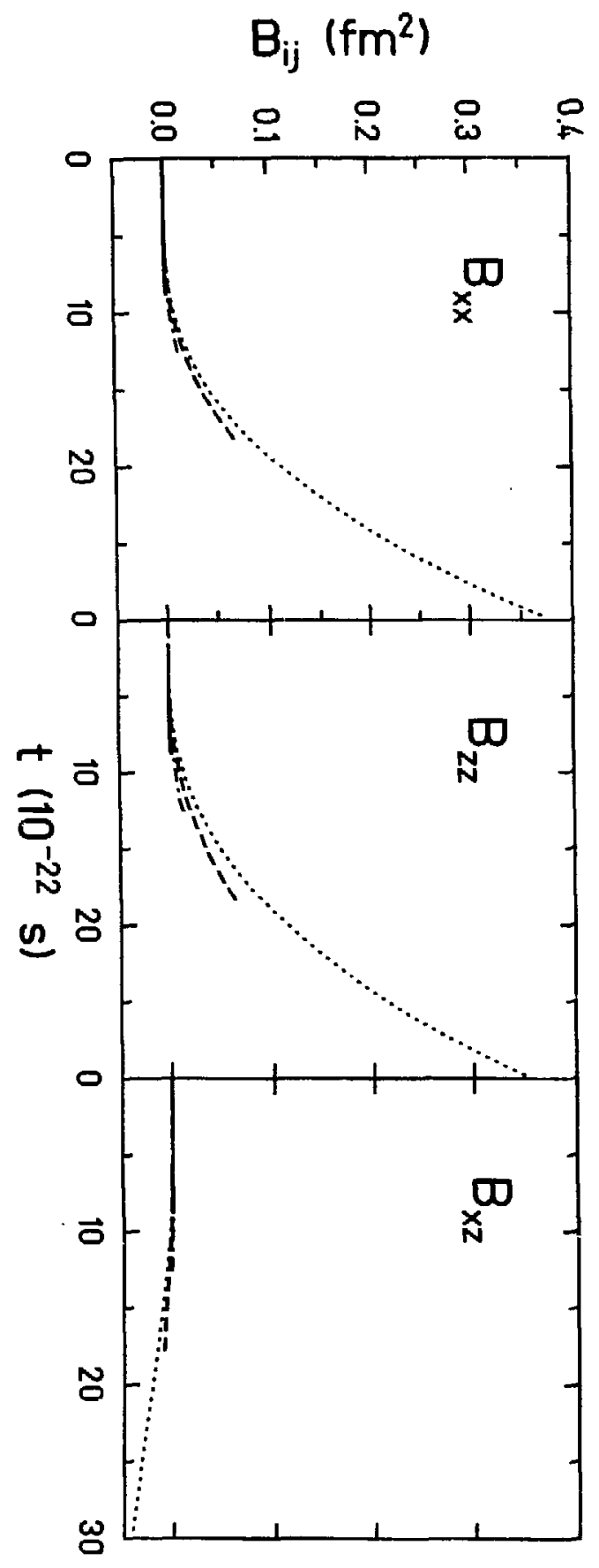

Fig. 3.7.7 Second moments of the spatial coordinates as a function of time for four different trajectories with the int tal

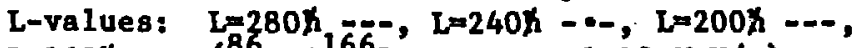
$\mathrm{L}=160 \mathrm{~K} \cdots\left({ }^{86} \mathrm{Kr}+{ }^{166} \mathrm{Er}, \mathrm{E}_{\mathrm{lab}}=8.18 \mathrm{MeV} / \mathrm{u}\right)$. 

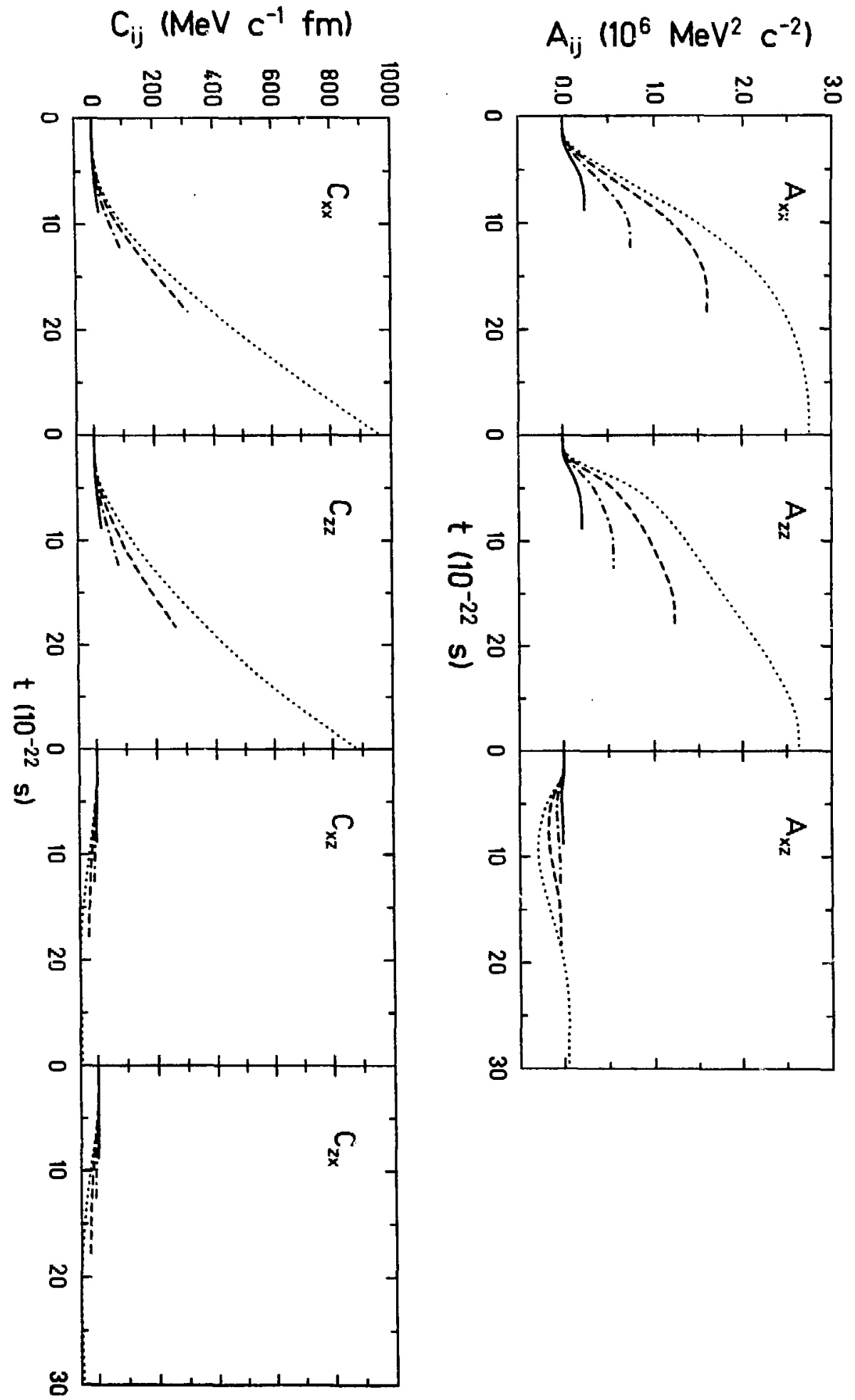

Fig. 3.7.8 Second moments of the relative momentum (upper part) and covariances between coordinates and momenta (lower part) as a function of time for four different trajectorles with the initial L-values: $L=280 \mathrm{~K}--, L=240 \mathrm{~K}-\cdots-$,

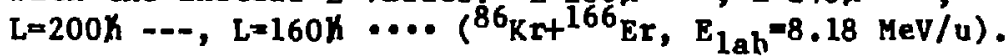


The Importance of the "velocity" part to the diffusion is measured by $\omega=\left|\Delta u^{+}\right| \rho_{F} / T(c, f . \operatorname{section} 3.5)$. Figure 3.7 .9 shows $\omega$ as a function of time for different trajectories. For $\mathrm{L}=280 \mathrm{~h}$ w stays throughout the whole interaction in the velocity regime. For $L=240 K$ it reaches the temperature regime for part of the reaction time. Only for $\mathrm{L}$ values below about $\mathrm{L}=160 \mathrm{~h}$ one would accept the Einstein relation (eq.3.5.30) to be a meaningful approximation. Even then, one misses the boost of the variances by $D^{\mathrm{U}}$ in the early stage of the collision.

We believe that in every theory whish determines diffusion coefficients for low energy nucleus-nucleus collisions one should take care of the velocity displacement of the single-particle distributions. This effect is being sacrificed frequently when the single-particle transition probabilities are discussed in terms of single-particle energy distributions instead of phasespace distributions.

Once the two nuclet are emerging from the interaction zone the diffusion tensor becomes zero and the time evolution of the variances is affected by the Coulomb interaction only. In an experimental setup one measures usually the final kinetic energy, $E_{f}$, and the scattering angle, $\theta_{f}$. Therefore, we hr re to transform the quantities resulting from the trajectory calculation to the observed ones. The mean values $\left\langle E_{f}\right\rangle$ and $\left\langle\theta_{f}\right\rangle$ are deter.nined by Coulomb trajectories which start with inital conditions given by $X, Z, P_{X}$ and $P_{z}$ at scission. In order to transform the variance matrix, $\Sigma^{s}$, which contains the matrices $A_{1 j}, B_{1,}$, and $C_{1 j}$, from the scission point to infinity where the counters are, we make use again of the small width assumption and keep linear terms only [RW80]. The variance matrix, $\Sigma^{f}$, of the observed quantities may be written as a linear tranformation of $\Sigma^{S}$ : 


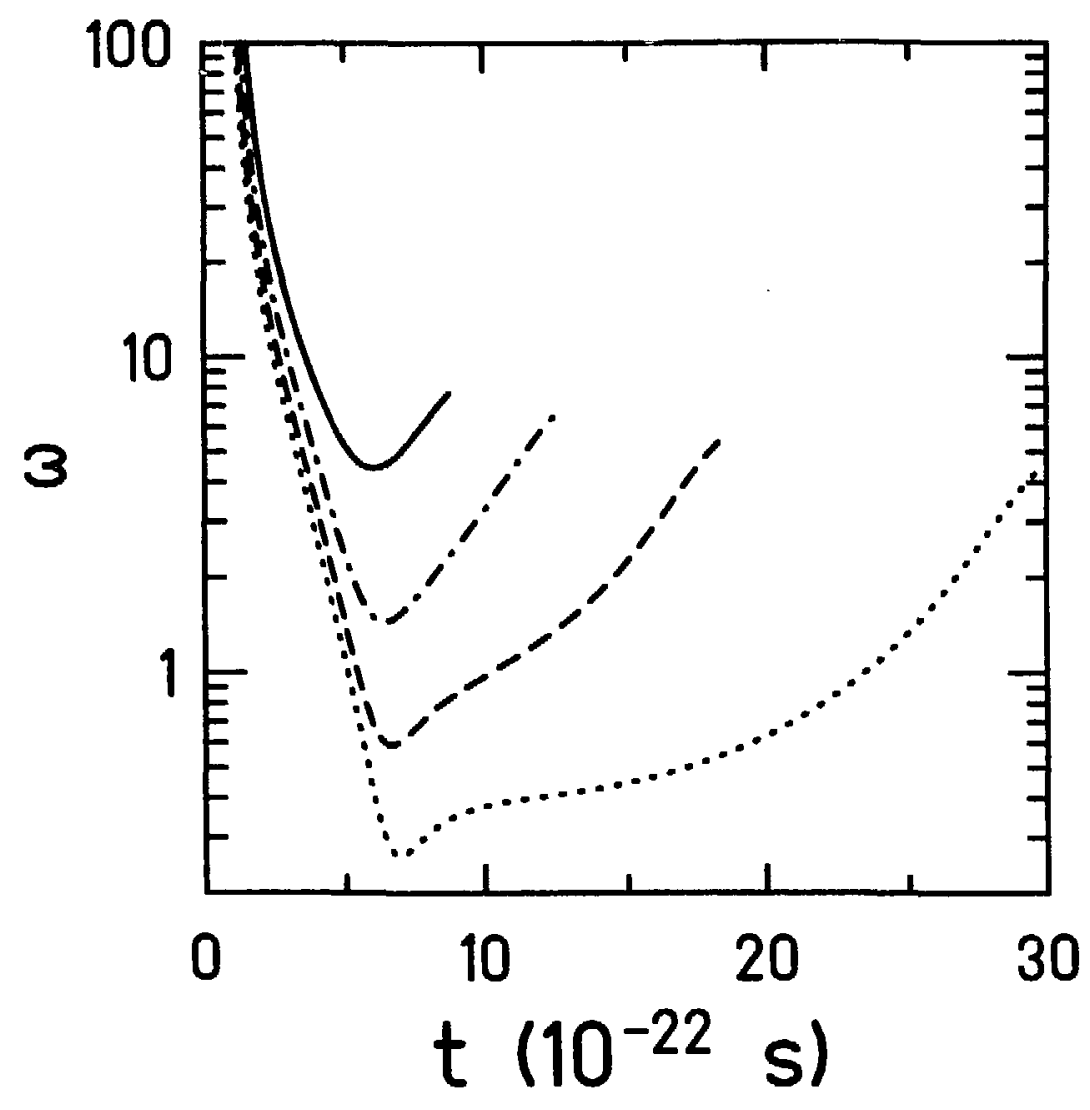

Fig 3.7.9 The ratio $\omega=|\vec{u}| \mathrm{mv}_{F} / \mathrm{T}$ which measures the relative contribution of the nonthermal "velocity part" as a function of time for four different L-values:

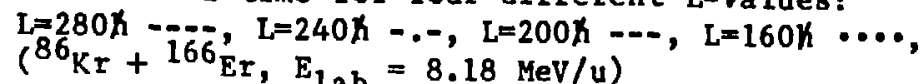




$$
\Sigma^{f}=T \Sigma^{s} T^{T}
$$

where the transformation matrix, $T$, is given by

$$
T_{1 j}=\left\langle\partial q_{i}^{f} / \partial q_{j}^{3}\right\rangle
$$

Here, $q^{s}=\left\{q_{1}^{s}\right\}=\left\{x, z, p_{x}, p_{z}\right\}$ denote the variables at scission, whIle $q^{f}=$ $\left\{q_{f}^{f}\right\}=\left\{E_{f}, \theta_{f}\right\}$ are the final observables. The dependence of $q^{f}$ on $q^{s}$ is given by the coulomb interaction and the derivatives are taken at the mean values. Wi th the help of this transformation the width in the energy 10ss, $\sigma_{\mathrm{EE}}$, and in the scattering angle, $\sigma_{\theta \theta}$, are calculated. They are shown in fig. 3.7.4 as the difference between the dashed and the solid lines. The width, $\sigma_{L L}$, of the $;$-component of the final relative angular momentum is given by

$$
\sigma_{L L}^{2}=\left\langle\left(1_{y}-\left\langle 1_{y}\right\rangle\right)^{2}\right\rangle \text { with } 1_{y}=z p_{x}-x p_{z},
$$

The information about the phase-space distribution is contained in the first and second moments. Thus, the only meaningful ansatz for the distribution is a multi-dimensional Gaussian:

$$
f^{f}\left(q^{f} ; L\right)=\left[(2 \pi)^{n} \operatorname{det} \Sigma^{f}\right]^{-\frac{1}{2}} \exp \left\{-\frac{1}{2} \Delta q^{f}\left(\Sigma^{f}\right)^{-1}\left(\Delta q^{f}\right)^{T}\right\}
$$

w 1 th

$$
\Delta q^{f}=q^{f}-\left\langle q^{f}\right\rangle .
$$


The final distribution, $\mathrm{f}^{\mathrm{f}}$, is a function of the final variables, $\mathrm{q}^{\mathrm{f}}$, and depends on the initial angular momentum, $L$, via the mean values, $\left\langle q^{f}\right\rangle(L)$, and the variances, $\Sigma^{f}(L)$. The latter ones are obtained by the transformation (3.7.1). The double-differentfal cross section is an fncoherent sum over all Inital L-values:

$$
\frac{d^{2} \sigma}{d E_{f} d \theta_{f}}=\frac{\pi}{\mu E_{1}} \sum_{K} \int L d L f^{f}\left(E_{f}, \theta_{k} ; L\right),
$$

where the initial centre of mass energy is denoted by $E_{1}$ and the reduced mass by $\mu$, respectively. The sum over $k$ takes care of the fact that all measured scattering angles range between 0 and $\pi$. For a non-symmetric system $\theta_{k}$ is glven by

$$
k \pi+\theta_{f} \quad \text { for } k \text { even }
$$

$\theta_{k}=$

$$
(1-k) \pi-\theta_{f} \text { for } k \text { odd }
$$

Usually $k=0$ and $k=1$ are sufficlent to cover the whole range of the distribution.

The single differential cross sections are obtained by integrating the double differential ones.

In fig. 3.7 .10 we display the double-differential cross section $d^{2} \sigma / d E_{f} d \theta_{f}$ as a function of the final scattering angle for the system ${ }^{136} \mathrm{Xe}+{ }^{209} \mathrm{BI}$ at a beam eneergy of $10.5 \mathrm{MeV}$ per particle. The calculated one occupies the upper part, while the experimental cross section [Ww81] is shown in the center part of the figure. The dashed lines indicate the correlation between the mean value $\left\langle E_{f}\right\rangle$ and $\left\langle\theta_{f}\right\rangle$ as it results from the trajectory 


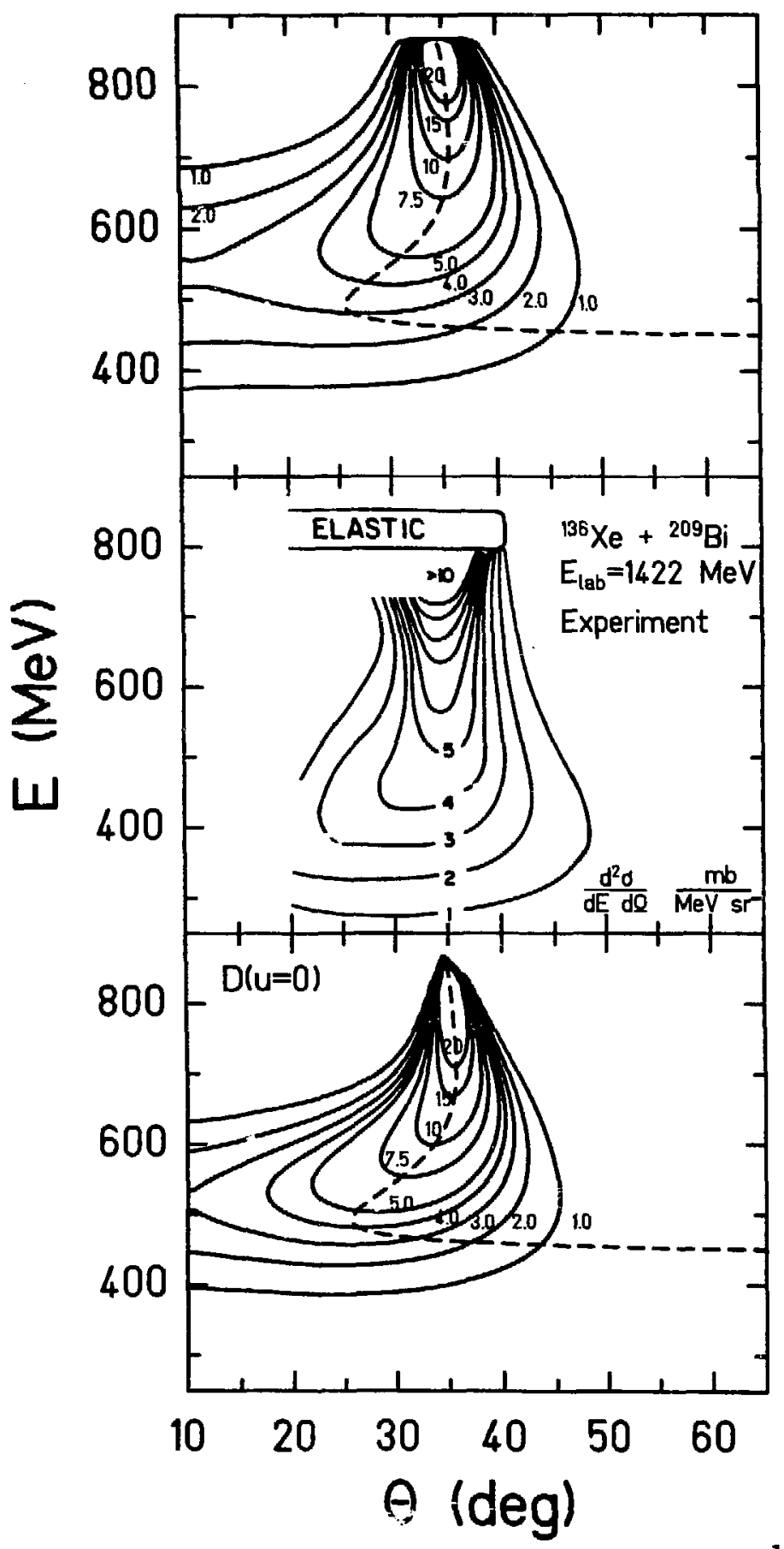

FIg. 3.7.10 Double-differential cross section for ${ }^{136} \mathrm{Xe}+{ }^{209} \mathrm{~B}$. Upfar part: result from the particle exchange model Including the nonequilibrium velocity part. Certre part: experimental cross section. Lower part: result of a calculation where the Einstein relation has been enforced by setting $\vec{u}=0$ at all times in the expression for the difiusion coefficient. 
calculation. The agreement for moderate energy losses is striking since the model reproduces the large spreading in scattering angle rather well. The fluctuations in this region, where the excitation energies and hence the temperatures of the nuclei are small, are mainly due to the velocity shift of the Fermi distributions whereas the temperature plays a negligible role. To illustrate the strong influence of the velocity part, $D^{\mathrm{l}}$, we calculated the Wilczynski diagram with a diffusion tensor obtained via the Einstein relation from the friction tensor (see eq. 3.5 .30 )

$$
D_{1 j}=T \quad Y_{1 j} \text { for }|\Delta \vec{u}|=0
$$

The resulting cross section is displayed in the lower part of fig. 3.7.10. We purposely set $\omega=0$ so that at all times the Einstein relation is enforced. By doing this the width in scattering angle collapses for small energy losses. What one anticipated from the considerations concerning $w$ becomes clearly visible in comparing the upper and lower part of $\mathrm{fig} \cdot 3.7 .10$. The velocity dependence of the diffusion tensor cannot be neglected.

The final energy for the completely damped events is not as low as the measured one. This is a well known shortcoming of trajectory calculations which do not include deformation degrees of freedom. By Inclusion of dynamical deformations the mean values of $E_{f}$ and $\theta_{f}$ follow the data to lower energies. 


\section{Dissipative Heavy Ion Collisions}

According to the classical trajectory picture shown in flg. 4.1 (from [N880]) one distinguishes three qualitatively different types of collisions. They belong to different impact parame'..rs, b, or equivalently to different angular momenta $1=b \sqrt{2 \mu E_{c m}}$ where $\mu 1 s$ the reduced mass and $E_{c m}$ the centerof-mass energy. The grazing Impact parameter, $\mathrm{b}_{\mathrm{gr}}$, is defined by the trajectory which just leads to a considerable nuclear interaction between projectile and target. For collisions with impect parameters larger than $b_{g r}$ one encounters direct reactions. For even larger impact parameters where the nuclear Interaction has vanished (distant collisions) target and projectile interact only via the coloms field. The concern of this lecture are the dissipative collisions which occur at impact parameters less than the grazing one. A comprehensive review on damped nuclear reactions can be found in "Treatise on Heavy Ion Science" edited by D. A. Bromley, Vol. 2 in an article written by W. U. Schröder and J. R. Huizenga [SH84].

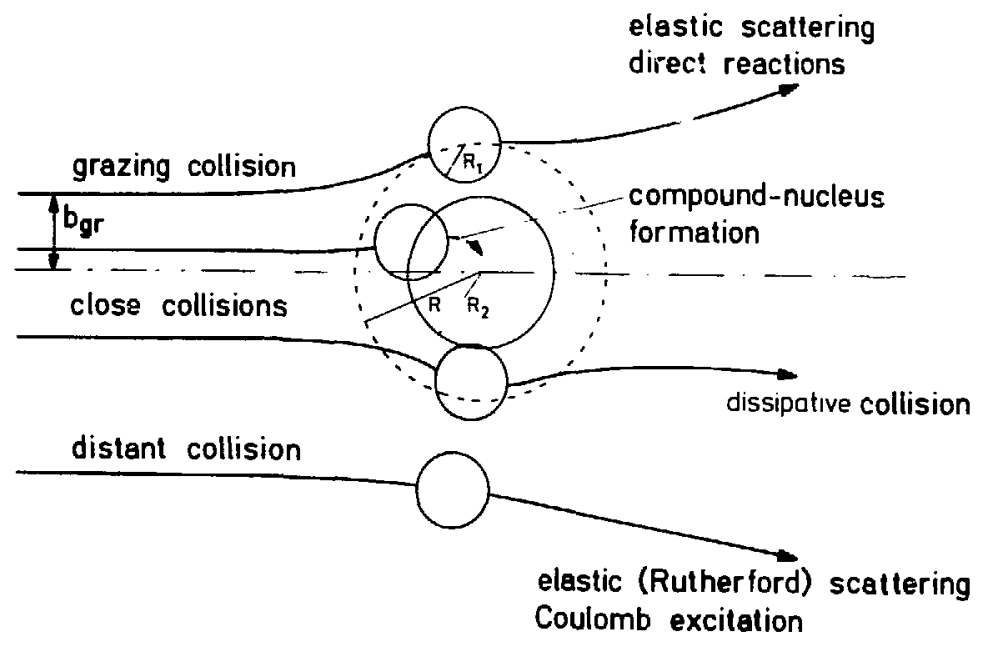

Fig. 4.1. Distant, grazing and close collisions in the classical picture of nucleus-nucleus collistons (from [N880]). Reproduced with permission of North-Holland Publishing Co. and the author. 
4.1. Quasielastic and deep-inelastic collisions

The contour plot of the double differential cross section $d^{2} \sigma / d E d \theta$, which is called Wilczynski diagram, displayed in fig. 4.1 .1 illustrates the continuous evolution of the two measured variables total kinetic energy, $E$, and scattering angle, $\theta$, from the center-of-mass energy of $456 \mathrm{MeV}$ and the grazing angle at $40^{\circ}$ down to the Coulomb barrier, $v_{b}$, and scatiering angles around zero degrees. The ridge in the cross section continues apparently to negative angles and en.rgies below the barrier. The distinction between quasielastic and deep inelastic collisions is not unique. It is suggested by the minimum in $\mathrm{d} \sigma / \mathrm{dE}$ seen in $\mathrm{fig} \cdot 4.1 .2$. In the trafectory calculation quasielastic events stem from those collisions where the trajectory does not led to Intimate contact of the two nuclei. In these reactions the proximity part of the particle-exchange friction is mainly responsible for the energy loss and the nuclei do not undergo large deformations. From a certain impact parameter down the nuclei merge and experience the opening of the neck degree of freedom. Instead of running up and down the binary valley the trajectories can move into the more flat landscape of stretched shapes with fat necks. In fig. 4.1 .3 we illustrate this for the ${ }^{86} \mathrm{Kr}+{ }^{166} \mathrm{Er}, \mathrm{E}_{\mathrm{cm}}=465 \mathrm{MeV}$ case with the aid of the $\mathrm{L}=280 \mathrm{~h}$ and $\mathrm{L}=240 \mathrm{~K}$ trajectories which are put on the potential energy landscape. 


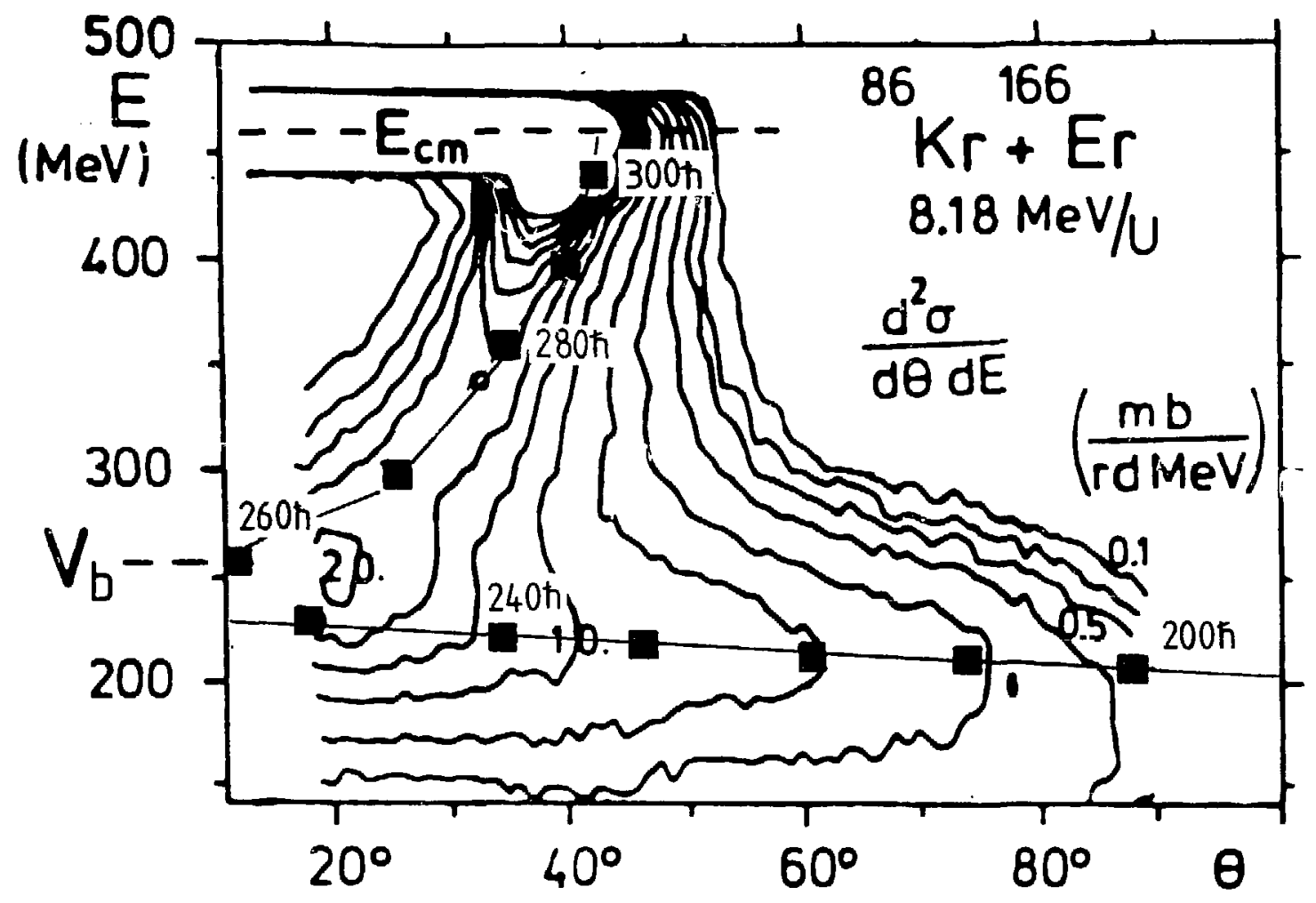

Fig. 4.1.1. Double differential cross section $\mathrm{d}^{2} \sigma / d E d \theta$ and mean values (full squares) resulting from the trajectory calculation. Experimental data from [GN80].

Reproduced from Heavy-Ion Collisions, Vol. 2 with the permission of North-Holland Publishing Co. and the author. 


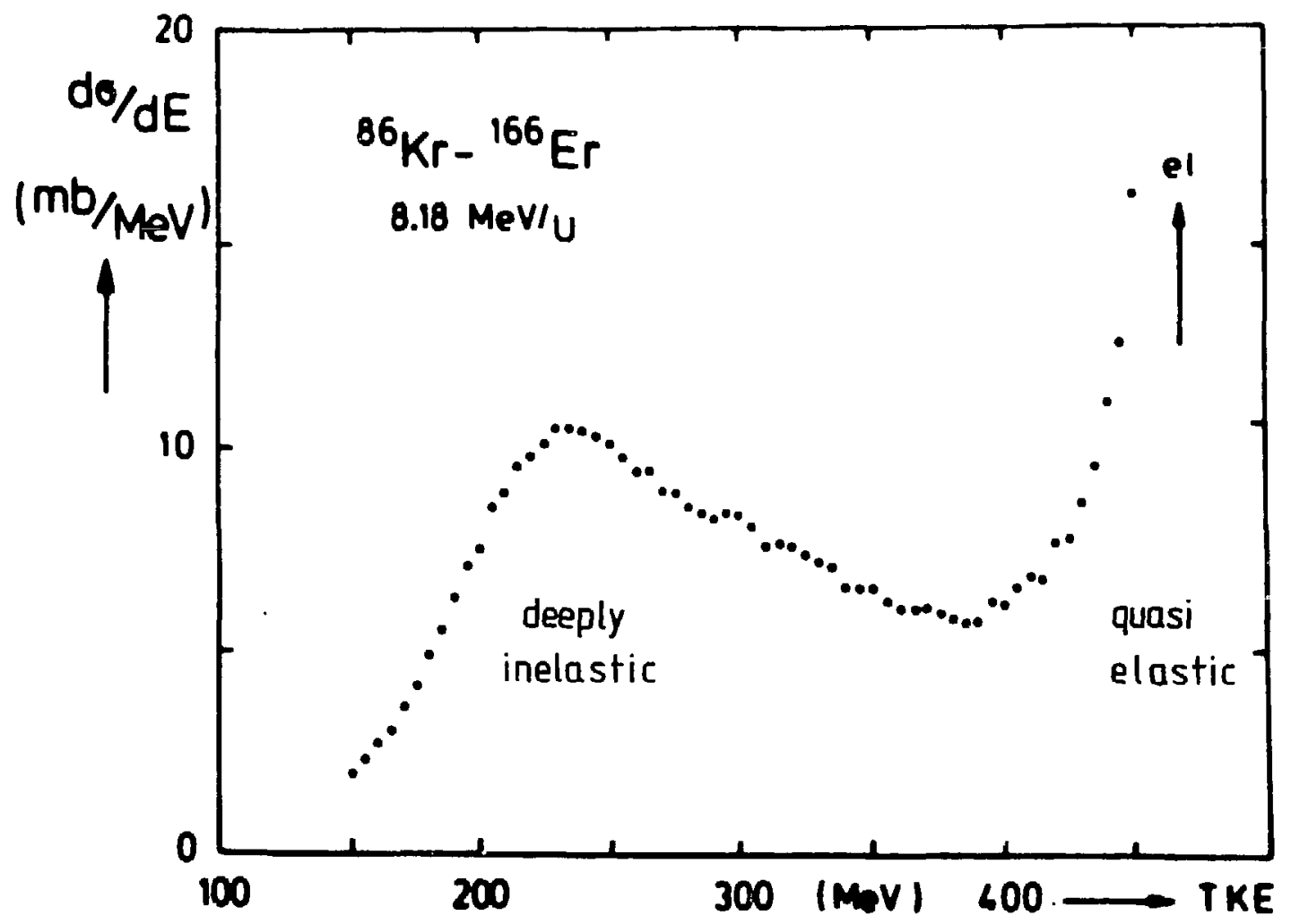

Fig. 4.1.2. Measured differential cross section do/dE for

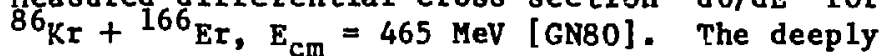
inelastic cross section is actually higher since the events with angles between 0 and 20 degrees are missing (c.f. fig. 4.1.1). Reproduced from Heavy-Ion Collisions, Vol. 2 with the permission of North-Holland Publishing Co. and the author. 


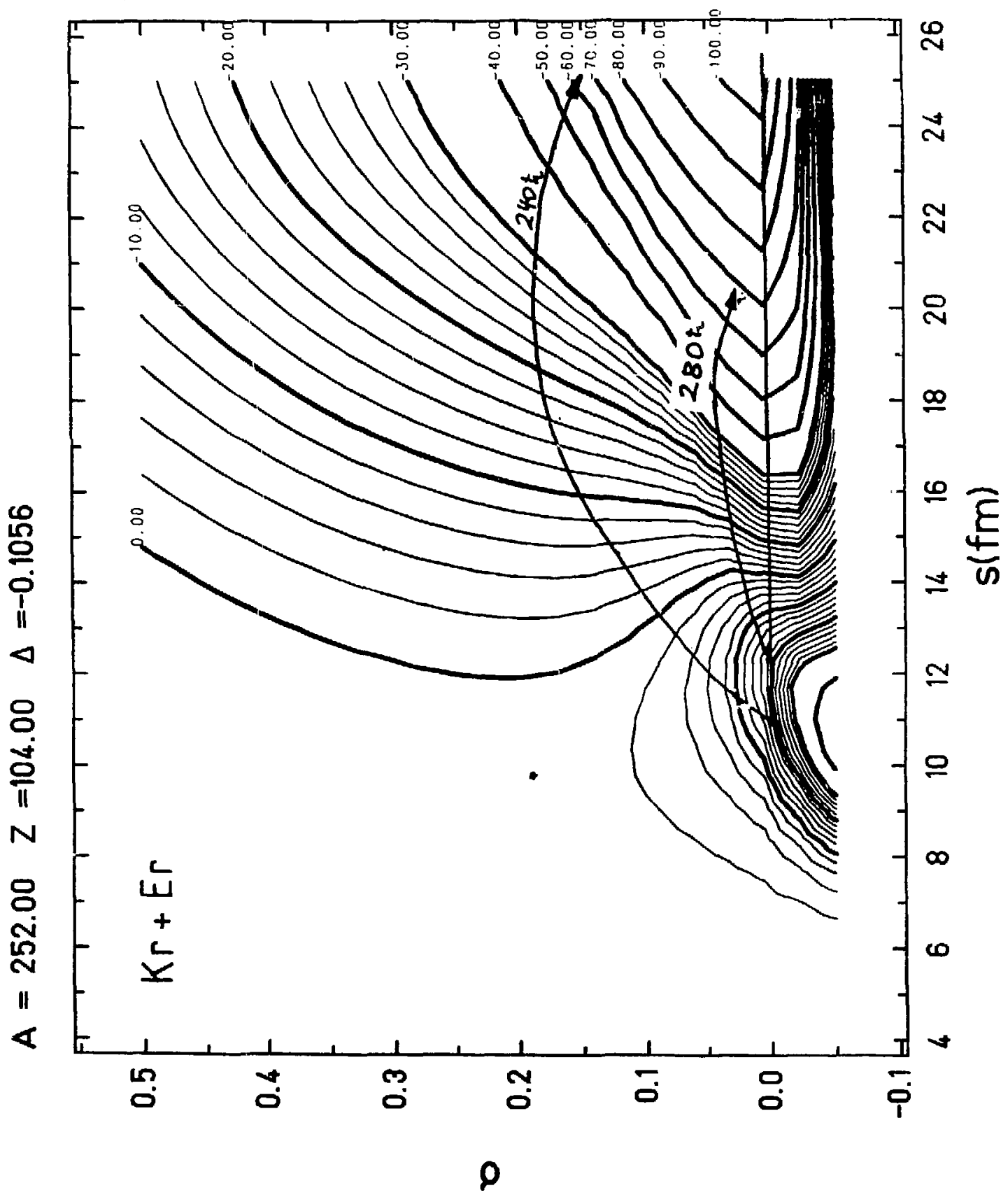

Fig. 4.1.3. Folding potential for a mass $A=252$ and charge $Z=104$ system at an asymmetry $\Delta=0.106$ corresponding to the $86 \mathrm{Kr}+166_{\mathrm{Er}}$ system. The energles are in MeV with respect to the compound nucleus. Included are two trajectorles for an Incoming L-value of $280 \mathrm{~h}$ and $240 \mathrm{~h}$, respectively. 
The trajectories have been calculated from the equations of motion (3.6.1) and (3.6.2) where the friction tensor $R_{1 f}$ contains Randrup's proximfty function. One should keep in mind that in addition to the potential energy the system is feeling different centrifugal forces for different $L$ values. The correlation between energy and angle is broadened by the statistical fluctuations. In fig. 4.1 .4 we show the Wilczynskl diagram as 1 t results from the model calculation which has been discussed in sections 3.5 and 3.7 . In this calculation the neck degree of freedom was frozen so that the nuclei cannot assume the stretched shapes necessary to reach final energles below the Coulomb barrier. Another effect of this restriction is that the quasielastic and the deep-inelastic events are not separated by a minimum as can be seen in fig. 4.1.5. For this and other reasons the dynamical evolution of the neck is necessary for a realistic description of dissipative heavy ion collisions. Deopite that deficiency fig. 4.1 .4 illustrates the strong fluctuations which come about by the nonequilibrlum part in the diffusion coefficients for the particle exchange model. Diffusion models which are based on the Einstein relation [RW80, BG78, BN78, SS81, FS83] predict appreclably smaller Eluc tuations. 


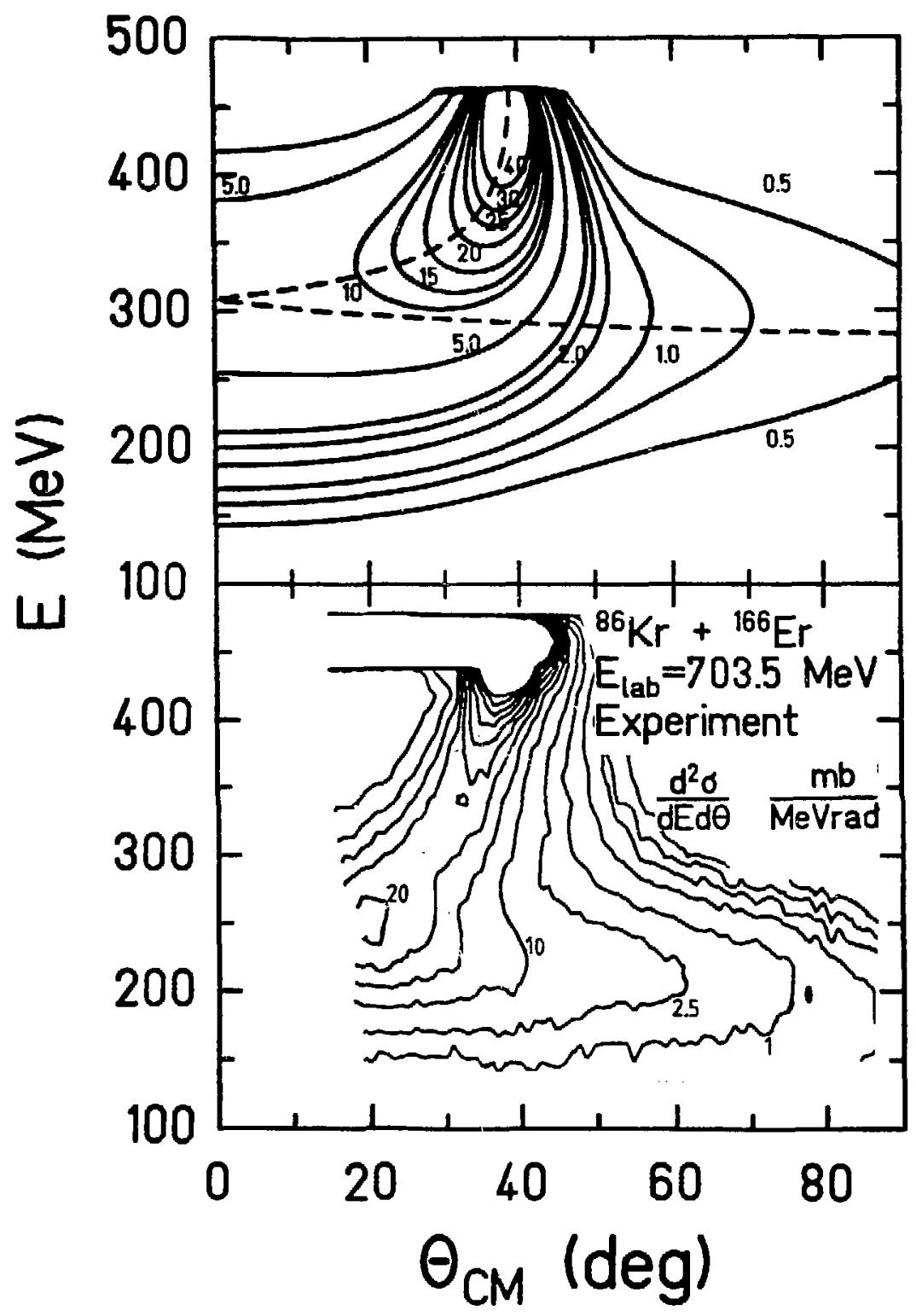

Fig. 4.1.4. Hilezynski diagram for ${ }^{86} \mathrm{Kr}+{ }^{166} \mathrm{Er}, \mathrm{E}_{\mathrm{cm}}=465 \mathrm{MeV}$. Upper part: result of the particle-exchange model. Lower part: experimental data from [GNBO].

Reproduced from Heavy-Ion Collisions, Vol. 2 with the permission of North-Holland Publishing Co. and the author. 


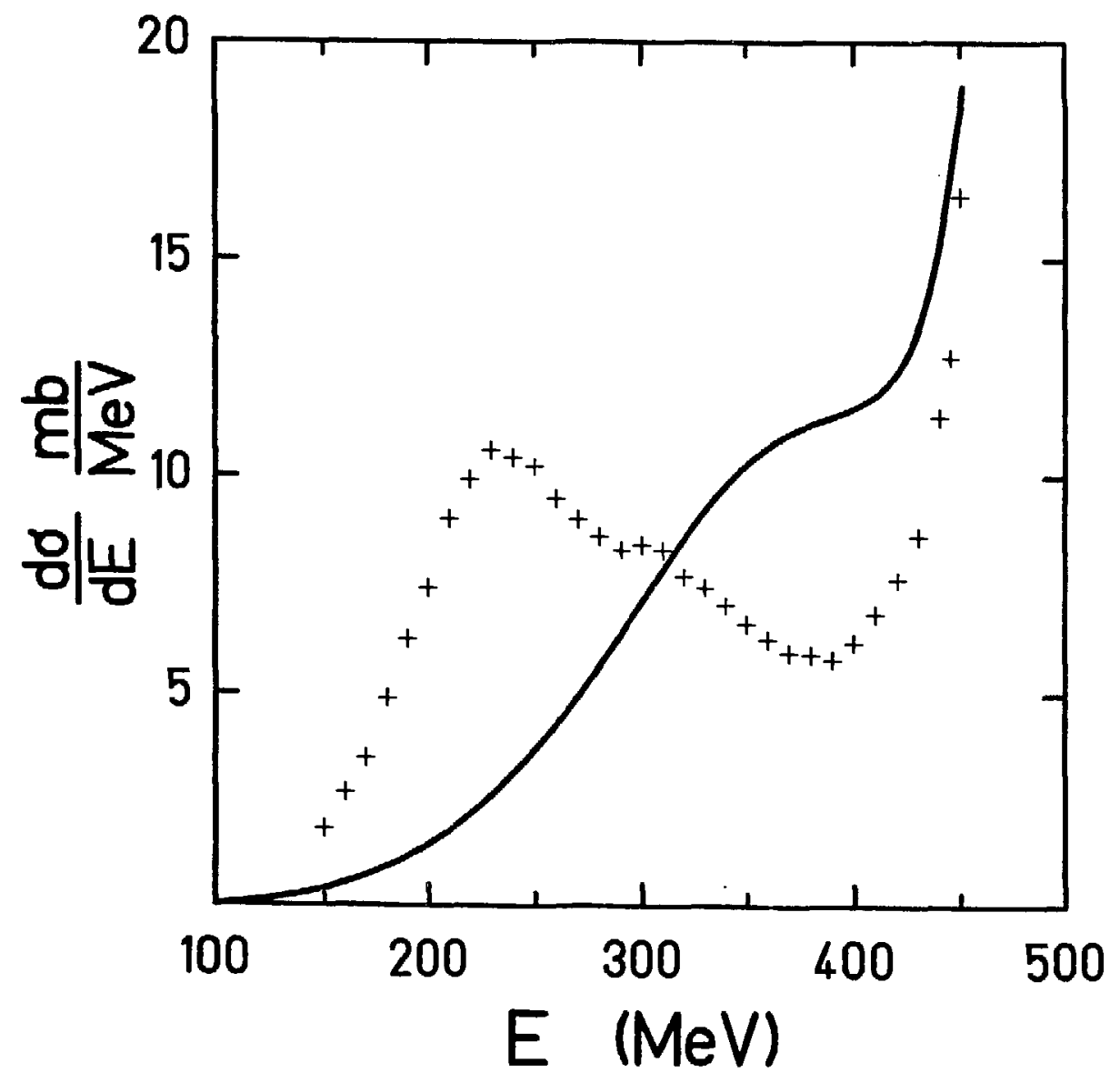

Fig. 4.1.5. Single differential cross sections for ${ }^{86} \mathrm{Kr}+{ }^{186}$ Er at $E_{\text {lab }}=8.18 \mathrm{MeV} / \mathrm{u}$ as a function of the final energy. The solid line displays the result of the calculation which does not include deformation jegrees of freedom while the crosses represent the measured data. 


\subsection{Large mass variance but sma11 mass drift}

As discussed in section 1, any dissipation in the collective variables inevitably goes along with fluctuations caused by the interaction with the Intrinsic degress of freedom. Bearing in mind the Einstein relation the question arises whether a small moblity of the mass number means that the mass distributions are peaked sharply. Looking back at fig. 3.5 .2 it becomes evident that not only the temperature opens phase space for particle transfer but also the mismatch in velocities is able to allow for many transitions which yleld large diffusion coefficients. If one neglects the difference in temperatures and chemical potentials, the diffusion coefficient for the mass asymmetry may be written as the sum of a "velocity" part and a "temperature" part proportional to the velocity mismatch and to the temperature, respectively (c.f. eqs. $(3.5 .33),(3.5 .34)):$

$$
D_{A A}=g_{A A}\left\{|\Delta \vec{u}| P_{F} \hat{D}_{A A}^{u}(\alpha)+T \hat{D}_{A A}^{T}(\alpha, \omega)\right\},
$$

where $p_{F}$ denotes the Ferml momentum. $\hat{D}_{A A}^{u}(\alpha)$ depends on the angle $\alpha$ between the window normal and the veloctty mismatch and varles between .3 and .2 for $\alpha$ between $0^{\circ}$ and $90^{\circ} . \quad \hat{D}_{A A}^{T}$ depends in addition on the ratto $\omega=|\Delta \vec{u}| p_{F} / T$ and ranges between 0 for $\omega=\infty$ and 1 for $\omega=0$. Only 1 .n the $1 \mathrm{im} 1 \mathrm{t}$ of $\omega+0$ where $|\Delta \vec{u}|$ is practically zero eq. $(4.2 .1)$ has the form of an Einstein relation: $D_{A A}=g_{A A} T$. Fox fllustrative purpose let us consider again the fictitious case of a central collision of two equal nuclel and assume that all enargy 1088 turns into heat. Herewith, the velocity mismatch and the temperature are related via the conservation of total energy by $E_{0} / A=E^{*} / A+m|\Delta \vec{u}|^{2} / 8$ and $E^{\star} / A=T^{2} \pi^{2} /\left(4 \varepsilon_{F}\right)$. For this case $\left(E_{0} / A=1\right.$ $\mathrm{MeV}$ ) the dependence of the diffusion coefficient on the transition of 
collective energy into heat is shown in $\mathrm{fig} .4 .2 .1$. The scale for $\mathrm{D}_{\mathrm{AA}}$ is chosen for a typical window size corresponding to $g_{A A}=.5\left(\mathrm{MeV} 10^{-22} 8\right)^{-1}$. In contrast to the Einsteln relation (dashed line) the diffusion coefficient does not vanish as the temperature goes to zero; it incresses in strength by a factor 2 when $\mathrm{T}$ decreases from $3.6 \mathrm{MeV}$ to $0 \mathrm{MeV}$. This Indicates that the particle exchange mechanism results in large fluctuations even for peripheral collision where the nuclel are not heated up too much. Randrup has calculated the same coefficlent [RA78, RA78a] but by means of different approximations he has obtained a forn called generalized Einstein relation with an effective. temperature. With his expression we get the dotted line in fig. 4.2.1.

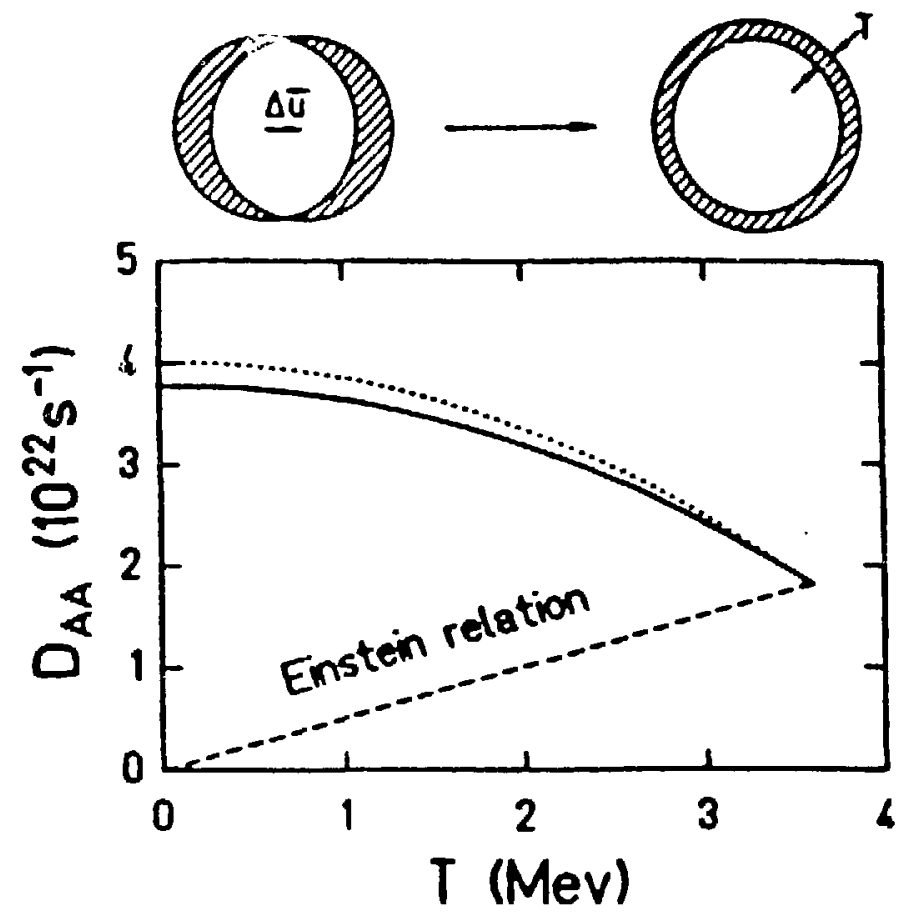

Fig. 4.2.1 Transition of the di-nuclear velocity distribution from the inftial nonequilibrium stage with a velocity mismatch $\overrightarrow{\Delta u}$ to a thermalized Fermi distribution with temperature $T$. Below, the corresponding change of the mass diffusion coefficient. The full line shows our result (eq. (3.5.33)) while the dotted line is Randrup's effective temperature multiplied with $g_{A A}$. The diffusion coefficlent resulting from the Elnstein relation lo proportional to the temperature (dashed line). 
Using the mass diffusion coefficient of eq. (3.5.33) in the trajectory calculation where $g_{A A}, \Delta \vec{u}, T$ and $\alpha$ are changing with time according to the course of development of the macroscopic varlables we obtain the varlance in mass number for each trajectory. The resulting correlation between mean energy loss and $\sigma_{A A}^{2}$ together with the mean mas drift is shown in fig. 4.2.2. The width of the mass distribution is dominated by the "velocity" part up to an energy 1088 of about $80 \mathrm{MeV}$. For these more per'spheral col.11sion the Einstein like diffusion coefficient results in much smaller variances which is In agreement with the small mass drift. Thus, the large varlance at small temperatures is due to a non-equilibrium phenomenon for which the Einstein relation does not apply. We have also added experimental data of Bohne et al. [BF83] although we are aware of the problem that due to fluctuations in the energy loss the cuts in $d^{2} \sigma / d E d A$ along constant $E$ do not result in the same width of the mass distribution as the one belonging to a particular incoming L-value which leads to the mean final energy E. (See subsection 4.4). 
(Reproduced with the permission of North-Holland Publishing Co. and the author)

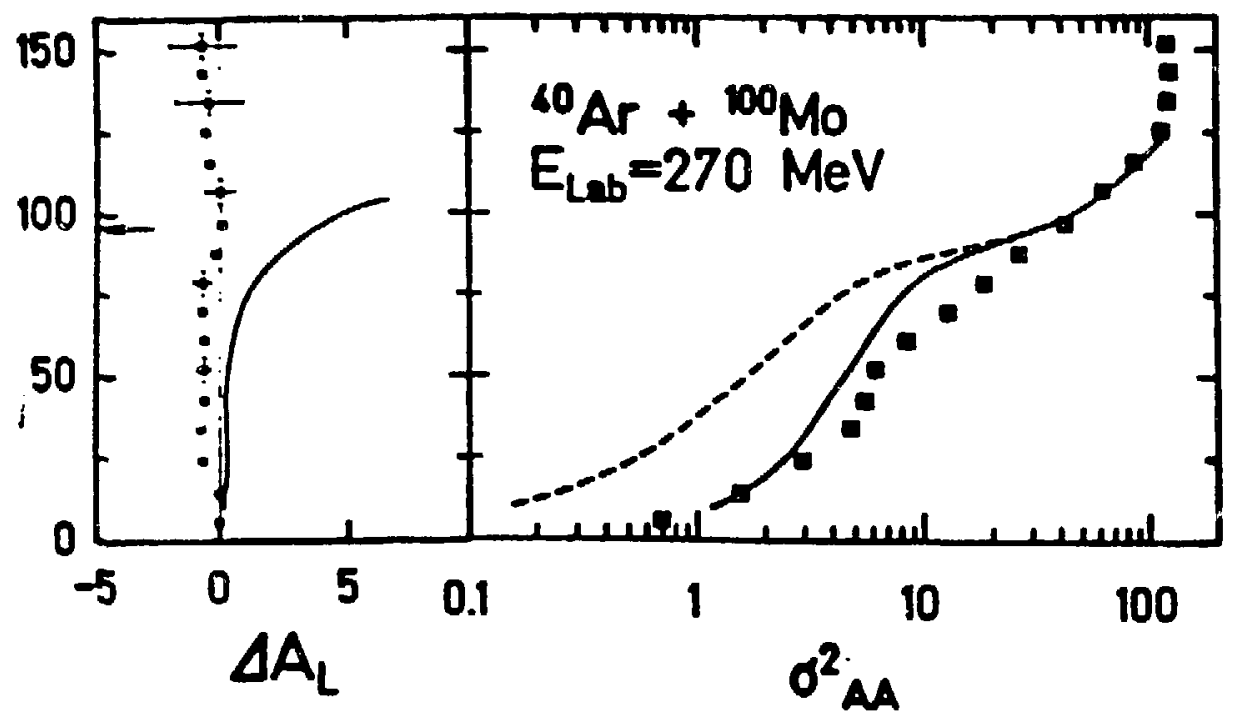

Fig. 4.2.2 Correlation between change of the mean mass number $\left(\Delta A_{t}\right)$, varlance of the mass number $\left(\sigma^{2}\right)$ and total kInetic energy loss (TKEL). The dashed line Indicates the varlance obtained with the Einstein relation $\left(D_{A A}=g_{A A} T\right)$. The arrow indicates the Coulomb barrier. Experimental data from [BF83].

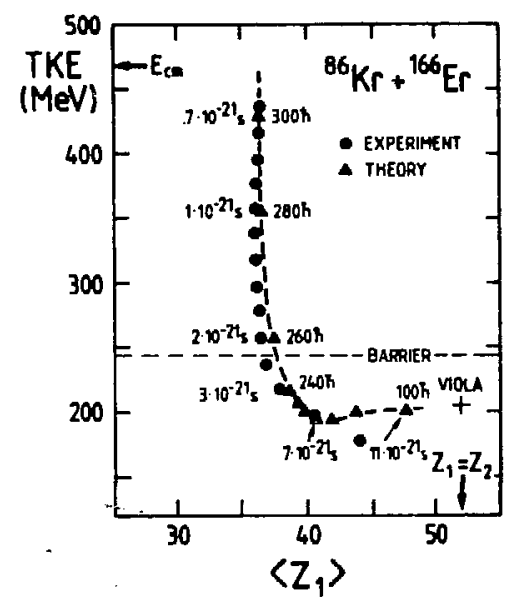

Fig. 4.2.3 Centrolds of the element distribution for the ${ }^{86} \mathrm{Kr}+{ }^{166} \mathrm{Er}$ reaction represented as a function of the total kinetic energy TKE. The experimental data are from [GG84]. The dashed Iine connects the triangles which result from the trajectory calculation. The cross marked by VIOLA is the energy for symmetric fission as calculated for the Viola systematics. Reproduced with the permission of Springer-Verlag Publishing Co. and the author. 
F'or the $\mathrm{Kr}+\mathrm{Er}$ case the charge drift has been measured at different bombarding energles [GG84]. The results of these experiments suggest that Independent of the Initial energy the mean charge does not drift as long as the final energy is above the Coulomb barrier. In $\mathrm{flg}$. 4.2 .3 the measured data are shown together with the results of the calculation for $\mathrm{E}_{1 \mathrm{ab}}=8.18$ $\mathrm{MeV} / \mathrm{u}$. The model reproduces this behavior. In the frame of the model $1 \mathrm{t}$ can be understood by considering the üuration of the colilsion in combination with the window size. For peripheral collisions the reaction time, Treac, which is also Included in $\mathrm{fig} .4 .2 .3$, is of the order of $10^{-21} \mathrm{~s}$. Futhermore, the effective window size $a_{w}$ is rather small during this time as the nuclei do not get into close contact. Therefore, the averaged mobility coefficient, $\int d t^{\prime} g_{A A}\left(t^{\prime}\right) / \tau_{\text {reac' }}$ is rather small and the driving force cannot move many particles through the window. However, once the nuclei come into intimate contact the window is widely open and in addition the reaction times grow, up to $10^{-22}$ s for the case shown. This allows for noticeable mass drifts seen at final kinetic energles below the Coulomb barrier of two touching spheres.

In the Ar + Mo case the measured mean mass number seems to be constant even at very large energy losses in contrast to the model prediction. Here, one has to keep in mind that the experimental data have been corrected for particle evaporation. A much better way wo" " be to complete the model by an evaporation calculation which takes as inpi: the model predictions. This has been done in [LV85]. We will discuss the:r results in section 4.4 .

\subsection{Sharing of excitation energies}

We have seen in section 3.7 (c.f. fig. 3.7.1) that a major part of the dissipated energy is produced by the window friction. The particle exchange mechanf.sm deposics about equal amounts of heat in each nucleus which means 
that the smaller nucleus has a higher temperature. On the other hand, as we derived in subsection 3.4 .2 there $1 \mathrm{~s}$ a flow of entropy or heat through the window which tends to equilibrate the temperatures. The trajectory calculation can tell us in how far this equilibretion can be achleved against the heat source window-friction.

From the center part of $\mathrm{fIg} \cdot 4.3 .1$ one sees that the model predicts a deviation from one for the ratio of temperatures, $T_{L} / T_{H}$, for all energies above the coulomb barrier (indicated by the arrow at $162 \mathrm{MeV}$ ). This is in contrast to the effective temperature extracted from neutron data as shown in the lower part of fig. 4.3.1. Also the large error bars on the extracted temperatures do not allow to plead the cause of agreement since other measurements of evaporated neutrons signal equal temperatures as well [TC79, CJ78, EG78]. However, recently Vandenbosch et al. [VL84] deduced from f18sion data a partition of the excitation energy which supports our and also Randrup's theoretical predictions. The ratio of excltation energies versus total kinetic energy loss is shown together with the theoretial numbers in fig. 4.3.2. Here, the experimental results are much closer to equal energles than equal temperatures. There is an obvlous discrepancy between the two different ways to measure the intrinsic excitation energy. This demands an explanation. 

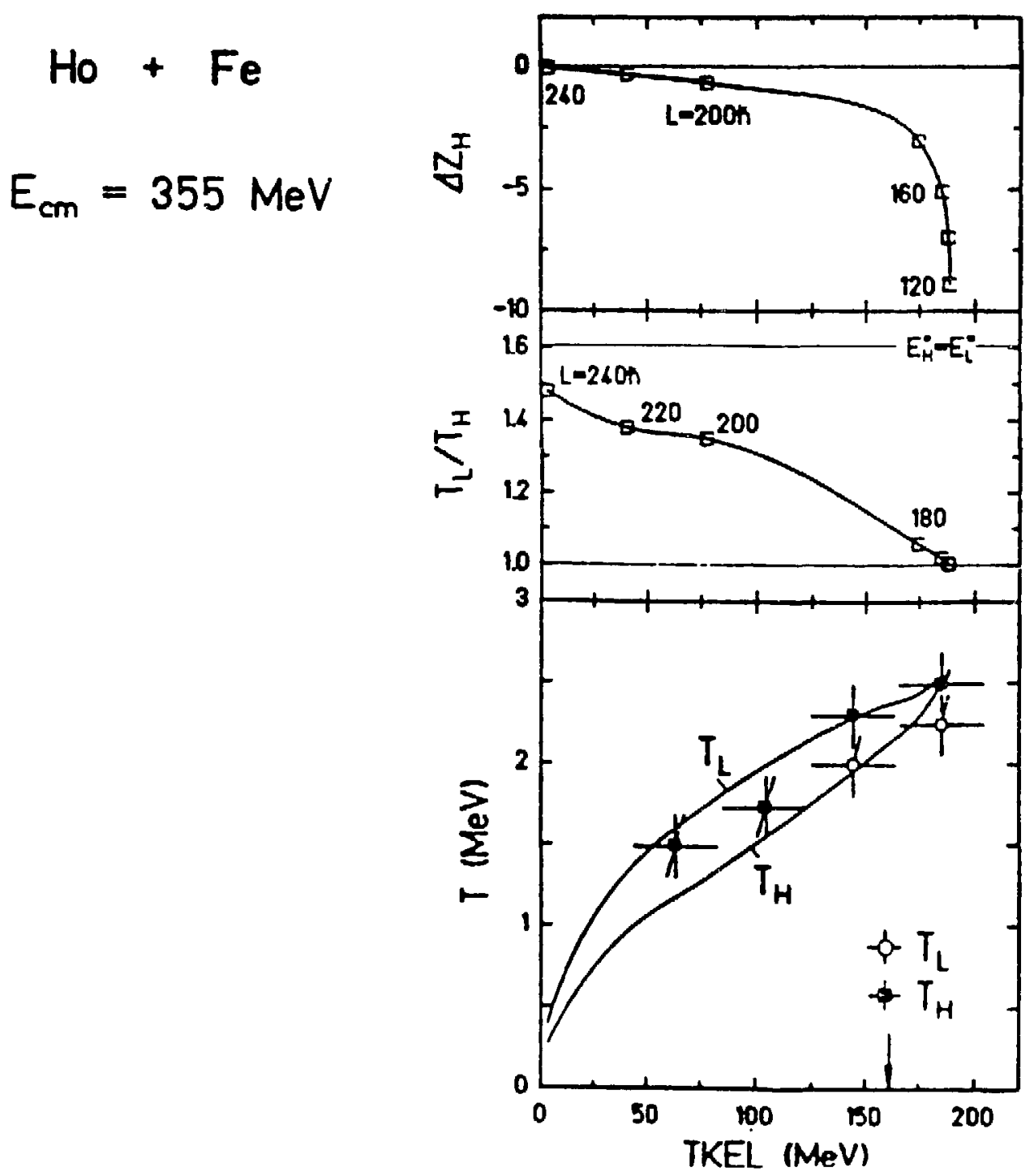

Fig. 4.3.1. Change of mean charge number, ratio of temperatures and effective temperatures ( $T=11 / 12 \sqrt{8 \mathrm{MeV} \mathrm{E}^{\star} / \mathrm{A}}$ ) as a function of energy 108s. Squares denote the incoming L-values. Experimental points are from [HB79]. The arrow indicates the Coulomb barrier. Reproduced with the permission of the American Physical Society and the author. 


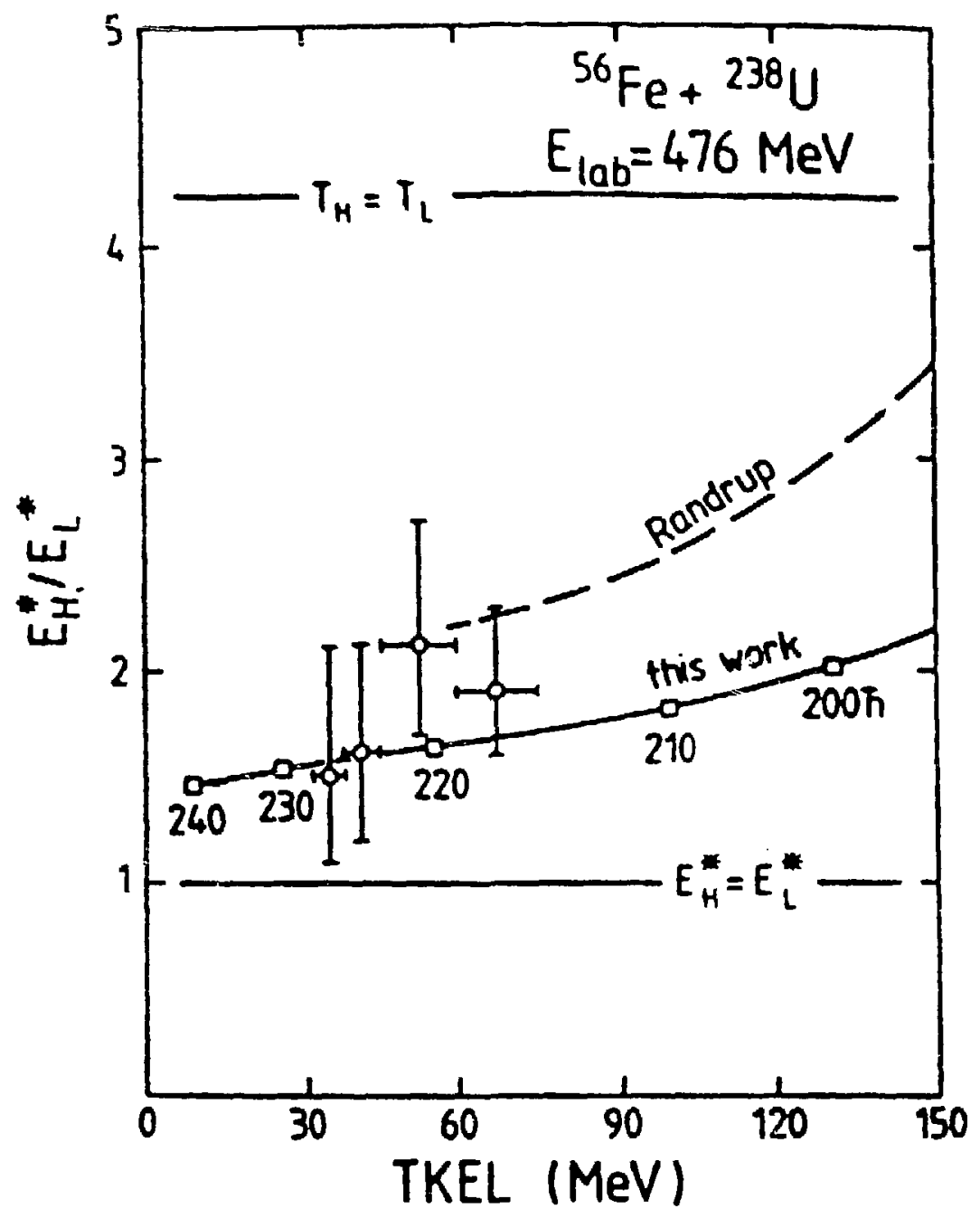

F1g. 4.3.2 Ratio of excitation energles between heavy and 1 ight fragment as a function of the kinetic energy 108s. The figure has been taken from the work of Vandenbosch et al. [VL84] and completed by adiing our result which is given as the full ine connecting the squares. The numbers Indicate the inftial Lvalues in units of $h$. Reproduced with the permission of the American Physical Soclety and the author. 


\section{$4.4 \quad$ Z-N distributions}

The distribution of the measured nuclel provides important Information about the dissipative mechanism especially about the dissipation caused by pa-ticle exchange. In most experiments it turns out that for final kinetic energles down to the Coulomb bairler the measured cross sections have a Gaussian shape In the charge, $Z$, and neutron number, $N$. Therefore, one considers usually the first and second moments of the distribution as a function of the energy loss

$$
\sigma_{A B}^{2}(E)=\langle A B\rangle-\langle A\rangle\langle B\rangle
$$

with $\langle 0\rangle=\int d N d z \frac{d^{3} \sigma}{\operatorname{dEdAdZ}} \circ\left[\int \mathrm{dNdZ} \frac{d^{3} \sigma}{\operatorname{dEdAdZ}}\right]^{-1}$

where $A$ and $B$ stand for the charge, neutron or mass number. From the defintion (4.4.1) follows the relation for the mass variance

$$
\sigma_{\mathrm{AA}}^{2}=\left\langle\mathrm{A}^{2}\right\rangle-\langle\mathrm{A}\rangle^{2}=\left\langle\mathrm{N}^{2}+\mathrm{Z}^{2}+2 \mathrm{NZ}\right\rangle-\langle\mathrm{N}+\mathrm{Z}\rangle^{2}=\sigma_{\mathrm{NN}}^{2}+\sigma_{\mathrm{ZZ}}^{2}+2 \sigma_{\mathrm{NZ}}^{2} \quad(4.4 .2)
$$

often one introduces the correlation coefficient

$$
\rho=\frac{\sigma_{\mathrm{NZ}}^{2}}{\sqrt{\sigma_{\mathrm{NN}}^{2} \sigma_{\mathrm{ZZ}}^{2}}}
$$

which gives the amount of correlation in the distribution. In the particle exchange model protons and neutrons are exchanged stochasticly without any correlation. Nevertheless, the nuclid distribution shows correlations due to driving forces. The potential landscape in which the diffusion takes place is governed by the valley of the nuclid masses. The potential energy surface for $\mathrm{Fe}+\mathrm{Fe}$ and $\mathrm{U}+\mathrm{Fe}$ is shown in fig. 4.4.1 (taken from [BM83]). 


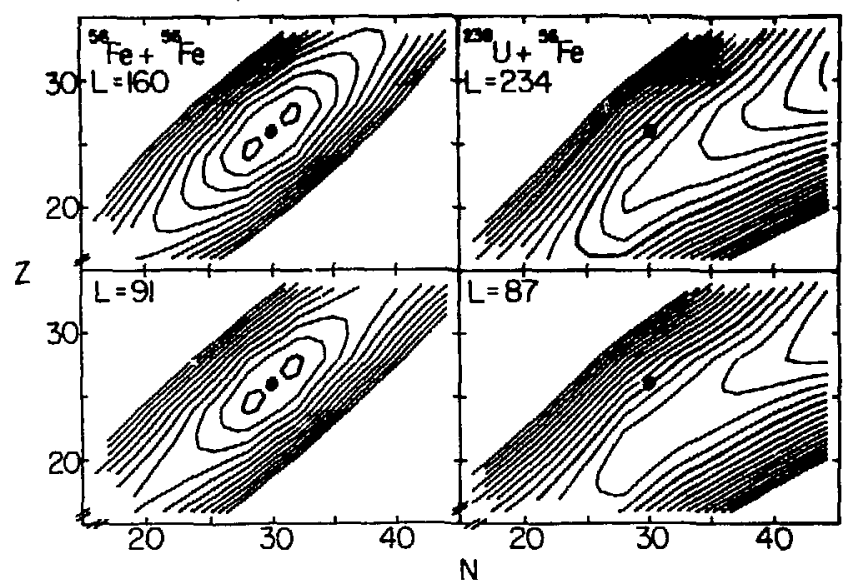

Potential energy surfaces for the $\mathrm{Fe}+\mathrm{Fe} \quad(l=160$ and $91 \hbar)$ and $U+\mathrm{Fe}(l=234$ and $87 \hbar)$ reactions. The calculations include shell corrections and are performed at a targetprojectile distance of $8.69 \mathrm{fm}$ for the Fe target and at $11.65 \mathrm{fm}$ for the $U$ target. The potentials are normalized so that $V=0$ (heavy line) at the injection point ${ }^{56} \mathrm{Fe}$, which is indicated by circles. The contour lines are $5 \mathrm{MeV}$ apart.

F1g. 4.4.1 From [BM83]. Reproduced with permisston of the American Physical Society and the author. 


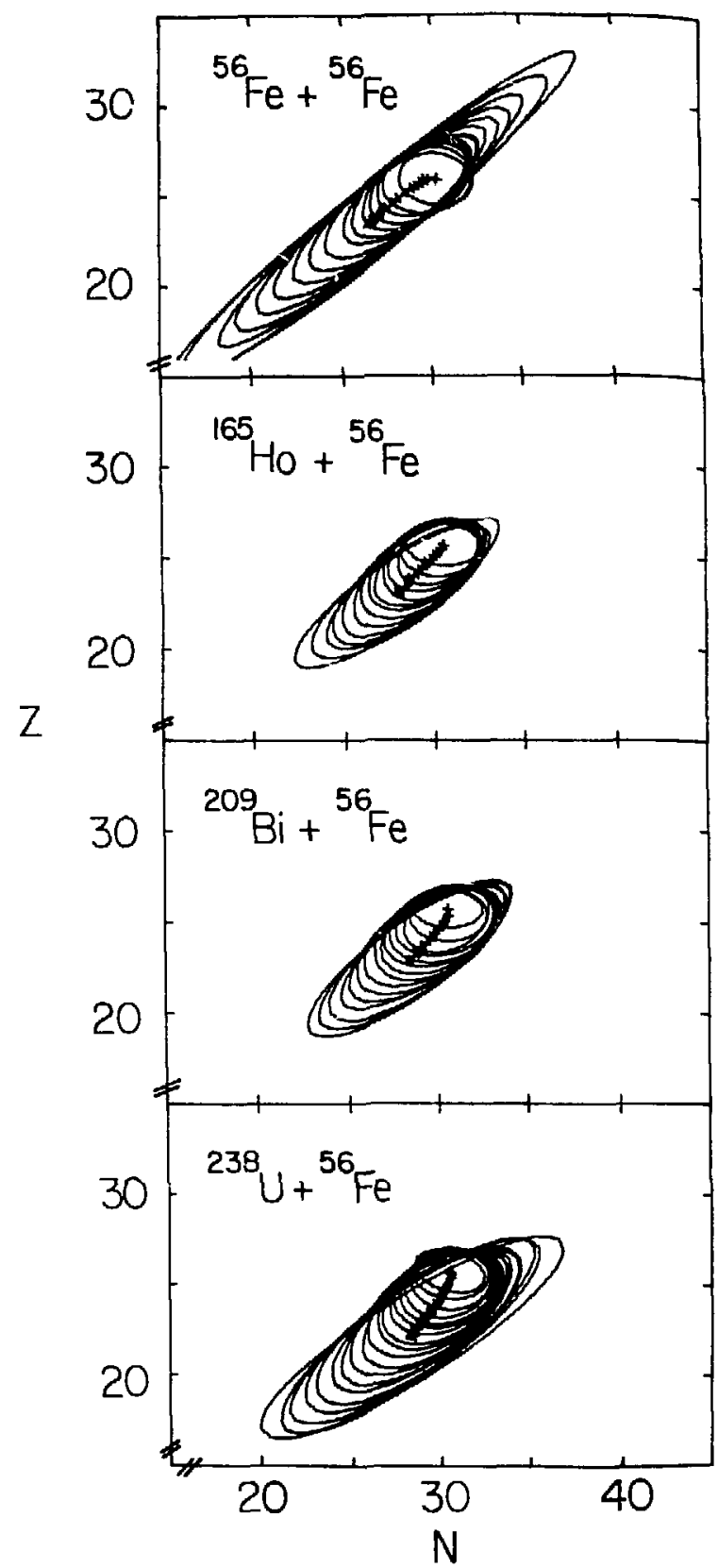

The contour lines with $P / h=e^{-1 / 2}$ for the neutron-proton distributions of damped reaction products at several energy lossen are shown for the reactions indicated. Starting with the smallest ellipse for each reaction, contour lines are shown for $12 \leq E_{L} \leq 60 \mathrm{MeV}$ in $8 \mathrm{MeV}$ steps and for $E_{L} \geq 60$ $\mathrm{MeV}$ in $10 \mathrm{MeV}$ steps. The crosses indicate the centroid of each ellipse. These contour lines represent an overview of the fit results given in Table $I I$ to $\mathrm{V}$ for the measured (not particleemission corrected) data.
Figure 4.4.2 Taken from [BM83].

Reproduced with the permission of the American Physical Society and the author. 
Fig. 4.4.2 [BM83] shows the "evolution" of the measured nuclid distributions ** as a function of the energy loss. Here, Breuer et al. performed a Gausian fit to the data [BY83] instead of using eq. (4.4.1). This is more reasonable because the width of the Gaussian fit corresponds better to the calculated variances. One sees an increasing width and a drift of the mean value with growing energy loss. However, the drift is malnly due to particle evaporation after the collision has taken place. The reason we picked this example out of the many experimental results (for a compilation see [FK84]) is that there is a recent calculation by Lock, Vandenbosch and Randrup [LV85] who performed trajectory calculations within the particle exchange model. In addition, they determined the effects of the particle evaporation by using an evaporation code to calculate the residue yield. The results are shown in $\mathrm{fig} \cdot 4.4 .3$. If one keeps in mind that this model, like the one discussed in section 3, has no adjustable parameters the agreement with the experimental data is gratifying. There is one exception: the mean values $\langle\mathrm{N}\rangle$ and $\langle\mathrm{Z}\rangle$ for the asymmetric system $\mathrm{Fe}+\mathrm{U}$. The drift in the neutron number goes in the wrong direction and the drift in the charge number is too large. The authors excluded a possible distortion by shell effects of the liquid drop potential energy which is used in the calculation. The landscape including shell corrections is shown in $\mathrm{fig} \cdot 4.4 .1$. From this one would also expect that $\langle\mathrm{N}\rangle$ Increases and $\langle Z\rangle$ decreases following the steepest descent. However, after correcting the data for neutron evaporation there seems to be no drift in $\langle N\rangle$. This problem which is not understood exists also in the case of Fe + Ho [BY83] and elsewhere [BN83]. A discussion can be found in the work of Griffin and Bronlowski [GB84]. There are other data (Xe + Fe in [SS81]) where the drift follows more closely the steepest descent as in the calculations [GH81]. 

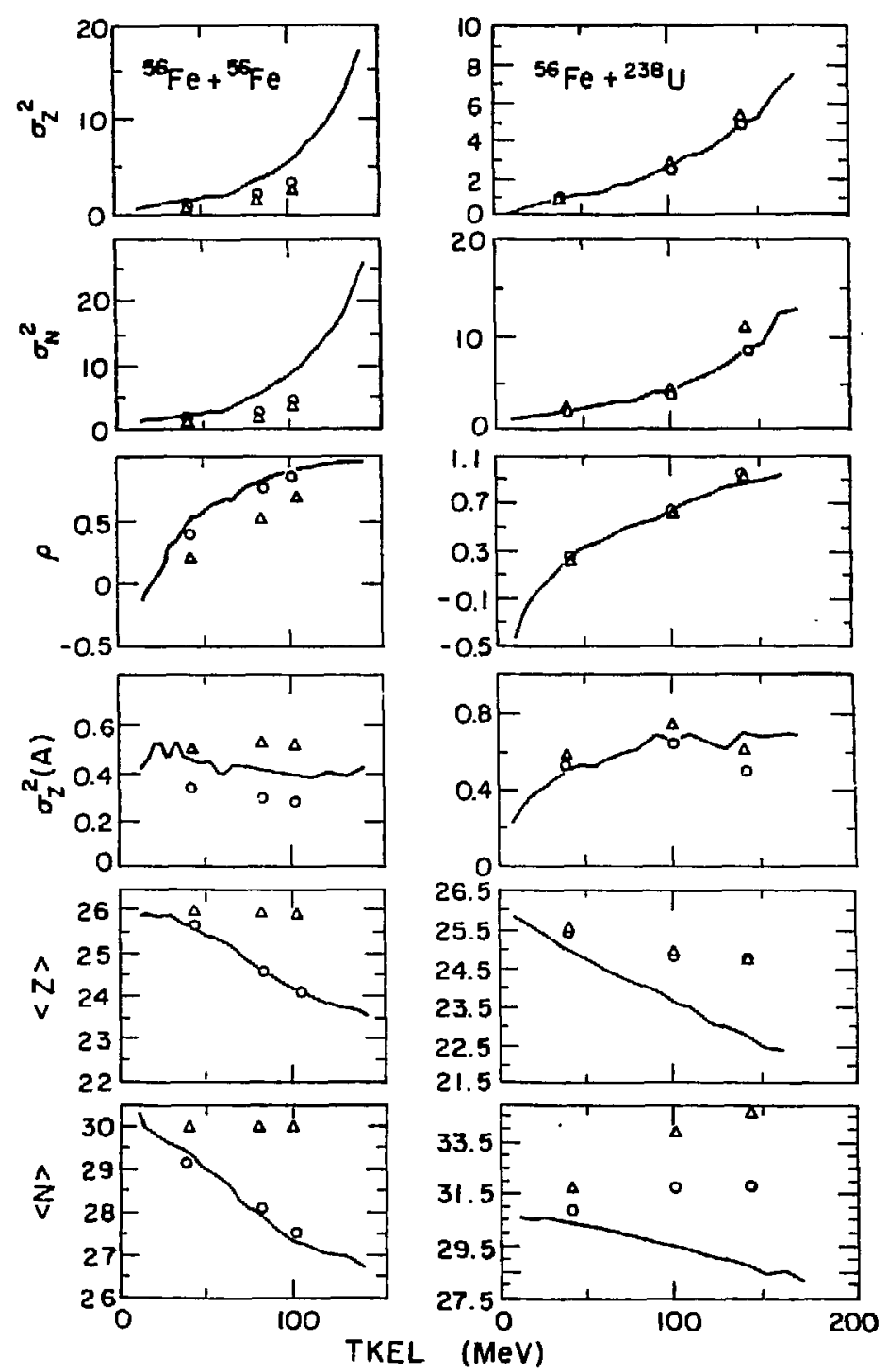

Fig. 4.4.3 Comparison of distribution parameters from the nucleon exchange model before $(\Delta)$ and after $(0)$ evaporation corrections. The full curves give the trends of the experimental data [BM83]. Figure is taken from [LV85]. Reproduced with the permission of the American Physical Society and the author. 
Another problem in comparing theory and experiment in graphs like the one :" In $\mathrm{fig} .4 .4 .3$ is that the measured quantity

$$
\sigma_{Z Z}^{2}(E)=\int d z \frac{d^{2} \sigma}{d E d Z}(Z-\langle Z\rangle)^{2} / \int d z \frac{d^{2} \sigma}{d E d z}
$$

is not the same as the calculated one. In the calculation each trajectory provides the width $\sigma_{\mathrm{ZZ}}^{2}(\langle E\rangle)$ via $\sigma_{\mathrm{ZZ}}^{2}(\mathrm{~L})$ together with $\langle E\rangle(L)$. The reason for this difference between $\sigma_{z z}^{2}(E)$ and $\sigma_{z z}^{2}(\langle E\rangle)$ is that due to fluctuations in the final energy different $L$ values contribute to the same final energy E. Therefore, $\sigma_{Z Z}^{2}(E)$ is a weighted superposition of many $\sigma_{\mathrm{ZZ}}^{2}(L)$ and can be quite different from the calculated $\sigma_{\mathrm{ZZ}}^{2}(\langle\mathrm{E}\rangle)$. In fig. 4.4.4 we show the calculated cross section $d^{2} \sigma / d E d z$ from which we deduce like in the experimental case $\sigma_{Z Z}^{2}(E)$ according to eq. (4.4.4). This is compared to $\sigma_{\mathrm{ZZ}}^{2}(\langle\mathrm{E}\rangle)$ which does not correspond to the measured varlance. ip to an energy loss of $250 \mathrm{MeV}$ they differ by about a factor of two. This discrepancy should be kept in mind when comparing $\sigma^{2}(\langle E\rangle)$ to experimental data as done in fig. 4.2.2. 


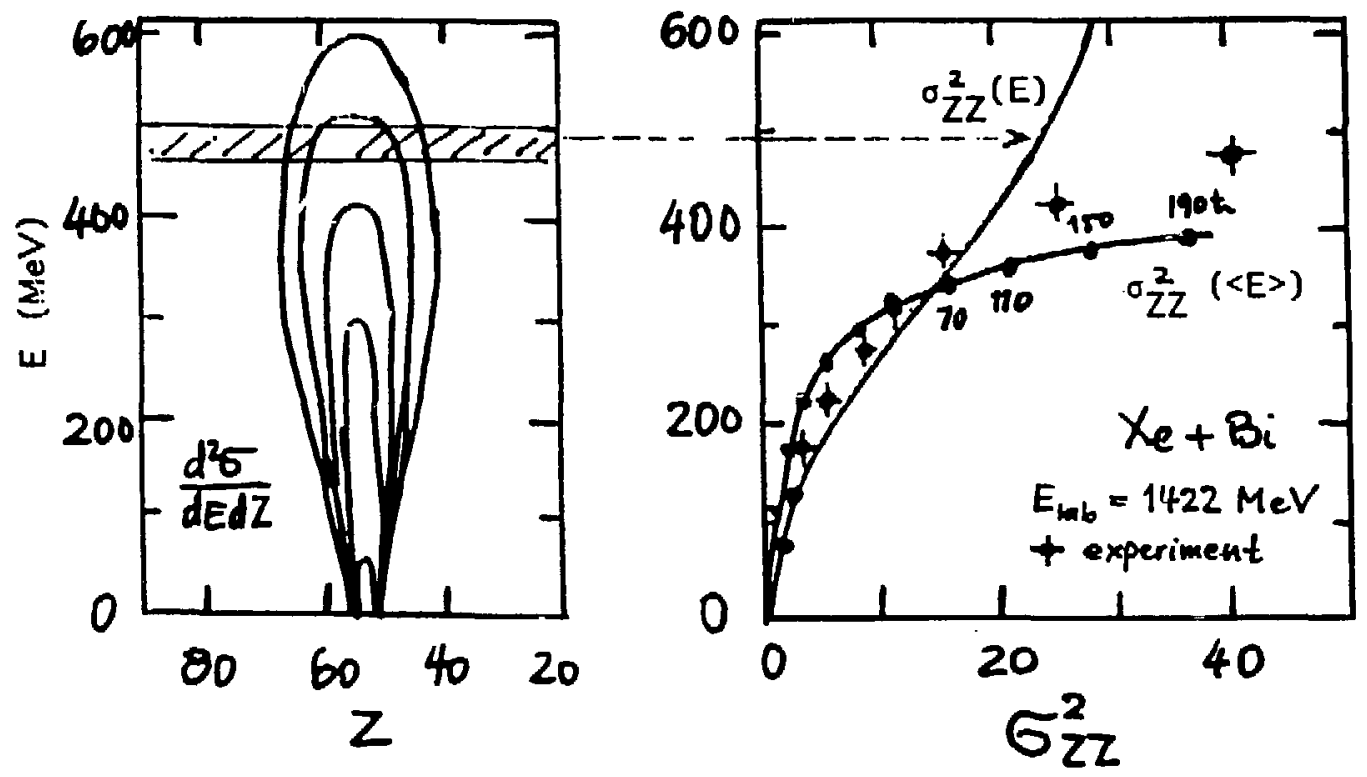

Fig. 4.4.4 The difference between $\sigma_{\mathrm{ZZ}}^{2}\left(\langle\mathrm{E}\rangle\right.$ ) and $\sigma_{\mathrm{ZZ}}^{2}$ (E) (from [FL84]). 


\subsection{Fusion}

For heary-ion collisions between nuclei with charges $z_{1}$ and $z_{2}$ that are small enough $\left(z_{1} z_{2} \lesssim 1600\right)$, the deep inelastic part of the cross section is followed by fusion of the two nuclei resulting in a compound nucleus which subsequently decays by particle evaporation or symmetric fission. The most simple and in many cases successful model considers only the effective nucleus-nucleus potential. We first will discuss this model in order to learn more about the dynamical aspects by looking at systems where the simple static model fails.

\subsubsection{The standard model}

In figure 4.5.1a we show the effective potential for the Ar + Ca system. The potential contains the folding potential as discussed in section 3.3 for shapes with $\sigma=0$ and $\Delta=0$, plus the rotational energy of the rigidly rotating system. Note that the potential barrier for relative angular momentum $\ell=0$ (denoted by $s_{b}$ ) lies outside the touching distance $\left(s_{t}\right.$ ) of the equivalent sharp surfaces. This is common for all light systems and one expects that the two nuclei once they have energy to overcome the barrier will feel the strong attraction and merge. They will not reseparate again if the frictional forces are strong enough to dissipate the collective energy before the trajectory comes back to the barrler. This strong absorption assumption allows us to calculate the fusion cross-section for low energies, as function of the barrier ueight $v_{b}$ and the position of the barrier $s_{b}$ (see eq (4.5.2)). To calculate the cross section one has to determine the angular momentum $\ell_{F}$ which gives a trajectory with its turning point at the barrier 


$$
\frac{\ell_{F}^{2}}{2 \mu s_{b}^{2}}+v_{b}=E_{c m}
$$

The angular momentum $\ell_{F}$ is related to the impact parameter via $l_{F}=\sqrt{2 \mu E_{c m}} b_{F}$ and tilerefore the fusion cross-section $\sigma_{F}=\pi b_{F}^{2}$ assumes the form given in eq. $(4.5 .2)$

If the angular momentum increases the pocket becomes more shallow and it finally disappears at 8 critical angular momentum $\ell_{c}$ (c.f. fig. 4.5.1a). At this point the centrifugal forces become stronger than the nuclear attraction; the nuclei cannot fuse for angular momenta higher than $\ell_{c}$. This provides the second boundary at higher bombarding energles (see eq. (4.5.3) and fig. $4.5 .1 \mathrm{b.})$

$$
\begin{array}{ll}
\sigma_{F}=\pi s_{b}^{2}\left(1-V_{b} / E_{c m}\right) & \text { for } v_{b}<E_{c m}<v_{b}+\ell_{c}^{2} / 2 \mu s_{b}^{2} \\
\sigma_{F}=\pi \ell_{c}^{2} / 2 \mu E_{c m} & \text { for } v_{b}+\ell_{c}^{2} / 2 \mu s_{b}^{2}<E_{c m}
\end{array}
$$

To summarize: the cross section for fusion of nuclei is at low energies IImited by the static properties of the entrance channel (height and position of the barrier); at high energies it is limited by the properties of the compound nucleus. One-dimensional pictures like fig. 4.4 .1 are not sufficient to determine $\ell_{c}$ because the collective variable $s$ in not choosen such that the equilibrjum shape of rotating nuclei is included. Rather we have to look for the disappearance of the fission saddle in the multidimensional energy landscape. See for example fig. 4.5.2 where besides the family of shapes, the 


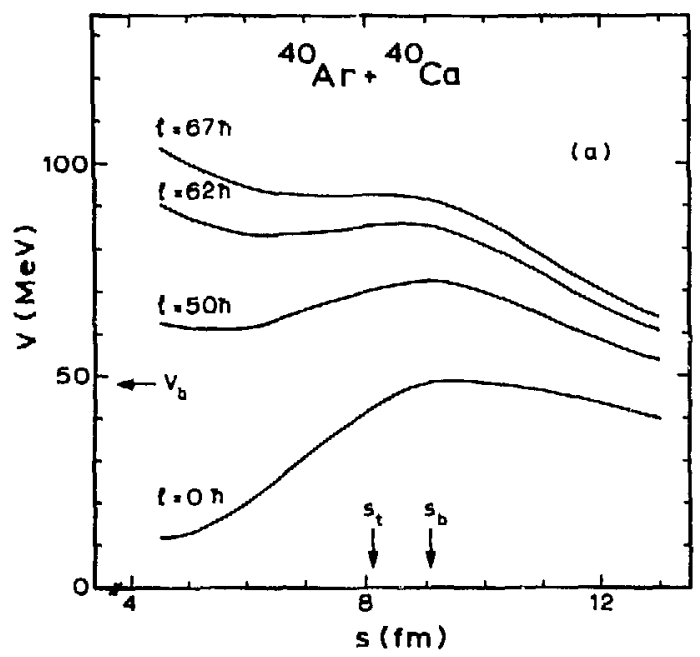

P.g. 4.5 .1 (a) Nucleus-nucleus plus centrifugal potential for different angular momenta.

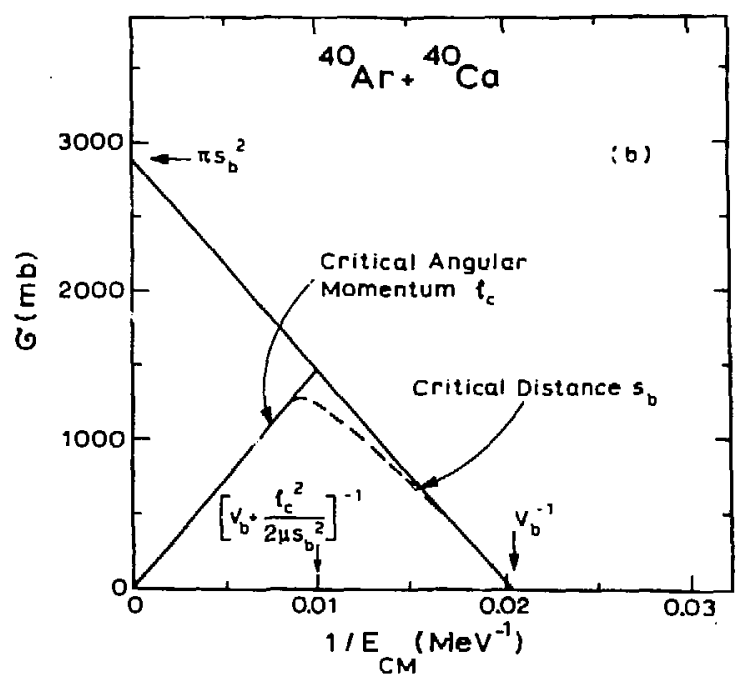

(b) Fusion cross section as a function of the inverse center-of-mass energy. The full lines denote the 1 imits as defined by the static properties of a one-dimensional potential. The dashed lines results from a trajectory model calculation using the potential shown in (a). 


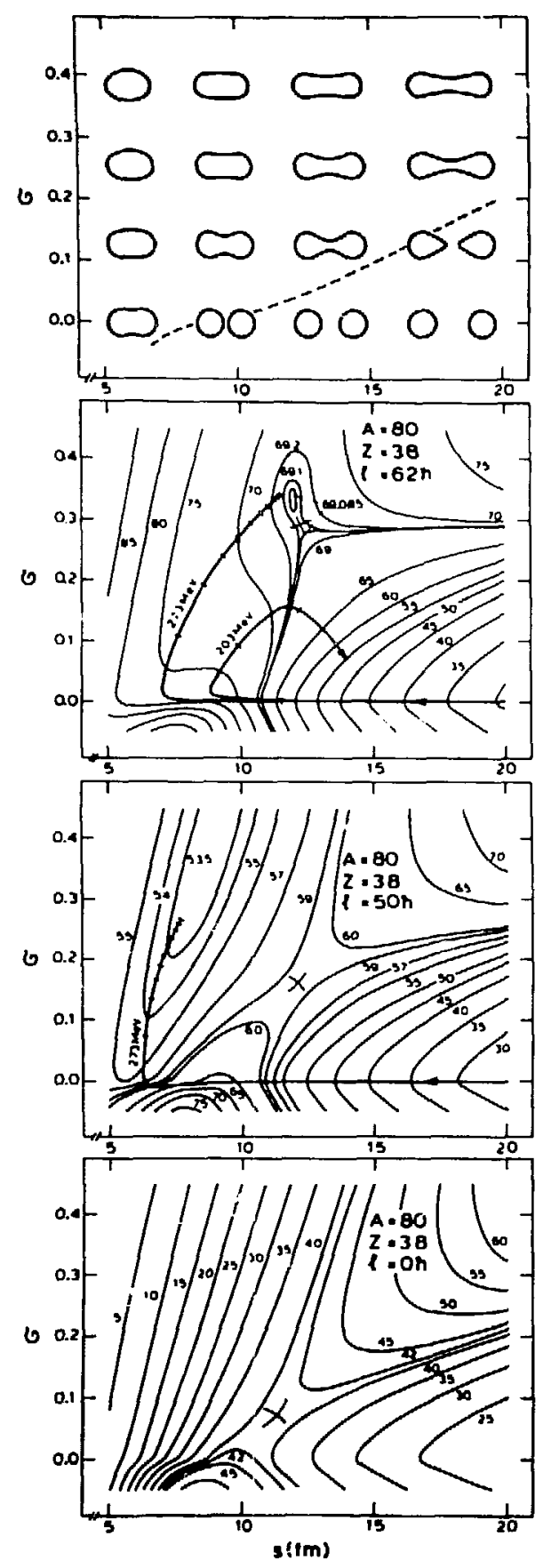

Fig. 4.5.2 Upper part: family of shapes for fixed asymmetry $(\Delta=0)$ as a function of $s$ and $\sigma$; scission is indicated by the dashed line. The other plots are potential maps for the varlous $\ell-$ values as indicated (contours in MeV)(from [CB83]). 
collective energy of the rotating nuclear system is shown for $\ell=0,50,62 \not$. With increasing angular momentum the saddle moves towards a minimum and disappears beyond $\ell_{B_{F}}=62 h$; compared $w 1$ th $\ell_{c}=67 \%$ as deduced from the one dimensional picture. Figure 4.5 .2 shows that the equilibrium shape of ${ }^{80} \mathrm{Sr}$ with $\&$ wh at $(s, \sigma)=(12.5, .33)$ is a stretched, slightly necked-in configuration.

How relevant the static considerations are for an actual trajectory calculation can be judged from fig. 4.5.1b where the dashed line shows the results of dynamical calculations using the one dimensional potentials shown in fig. 4.5.1a. Except for the top of the triangle where dynamics plays a role, the static considerations seem to be sufficient. This is also true for the calculations which include the neck degree of freedom. The results of

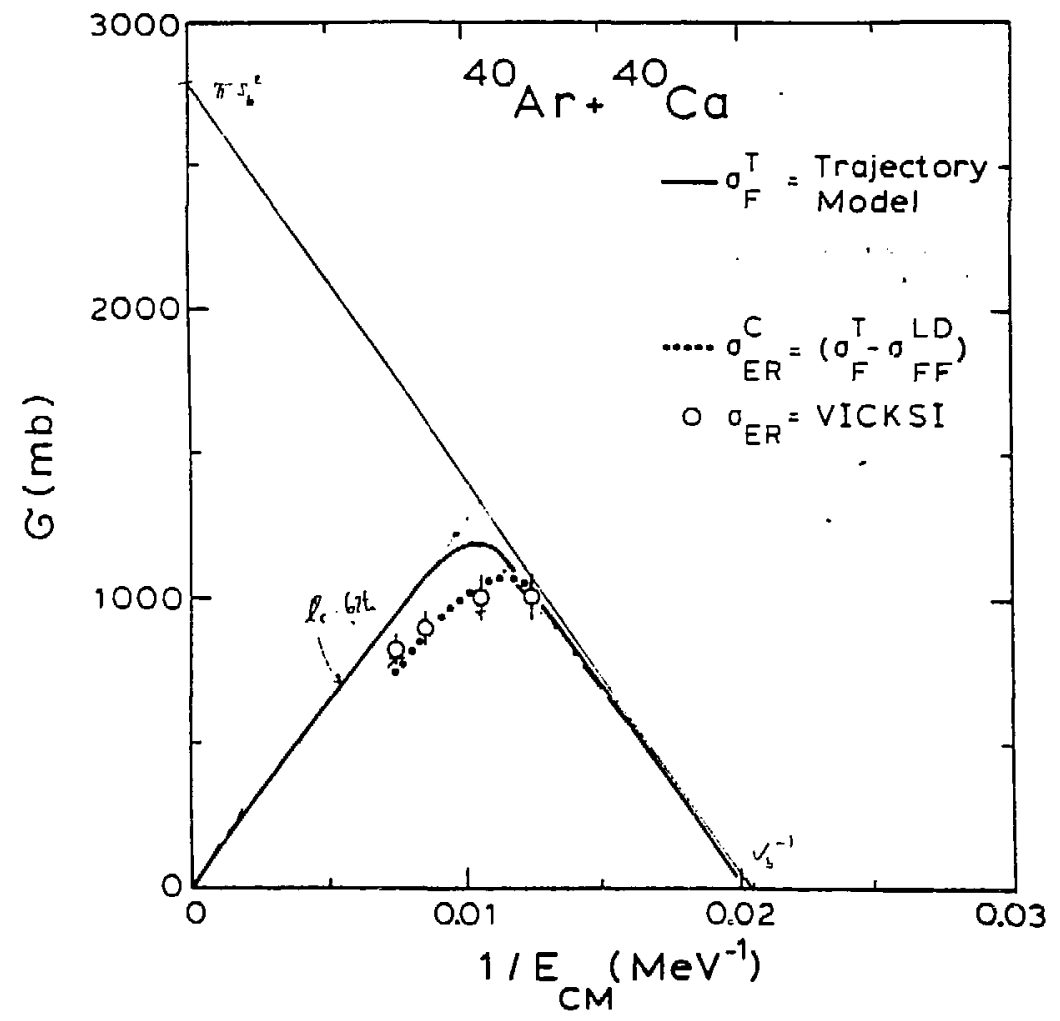

Fig. 4.5.3 Various cross sections (from [CB83]). 
this calculation are shown in $\mathrm{fig} .4 .5 .2$ as trajectories on the way to the equilibrium configuration and in $\mathrm{fig}$. 4.5 .3 as the cross section for forming a compound nucleus; all trajectories which get caught behind the fission saddle are assumed to contribute to the compound nucleus cross section. Figure 4.5.3 also shows that by subtracting the fission contribution from the calculated compound nucleus cross section one obtains the measured evaporation residue cross section.

The standard model can be applied successfully to many different systems. See for example the review article by Birkelund et al. [BT79]. However, there are deviations from this model due to three different dynamical effects which will be discussed next.

\subsubsection{Hindrance in the fusion of heavy nuclei}

The standard model breaks down when one wants to fuse heavy nuclei with $Z_{1} Z_{2} \gtrsim 1600$. Although there is still a pocket in the potential energy surface and the beam energy is high enough to bring the two nuclei into contact, they do not fuse; the strong absorption assumption is not valid any longer. Figure 4.5.4 shows measured fission like cross sections together with theoretical predictions based on the standard model (eqs. 4.5.2 and 4.5.3). Note that the critical angular momentum for the high-energy 1 imft (eq. 4.5.3) has also been deduced from the one-dimensional model. The critical angular momentum, $\ell_{B_{f}}=0$, where the fission barrier vanishes is much lower for these reactions. The measured fission-like cross section is higher than the cross section corresponding to $\ell_{B_{f}}=0$. Therefore, 1 t seems unlikely that a compound nucleus is formed which subsequently decays. The authors of [SB81] cal1 the cross sections shown capture cross section. It is actually the cross section for events which have final mass numbers around the mean value 


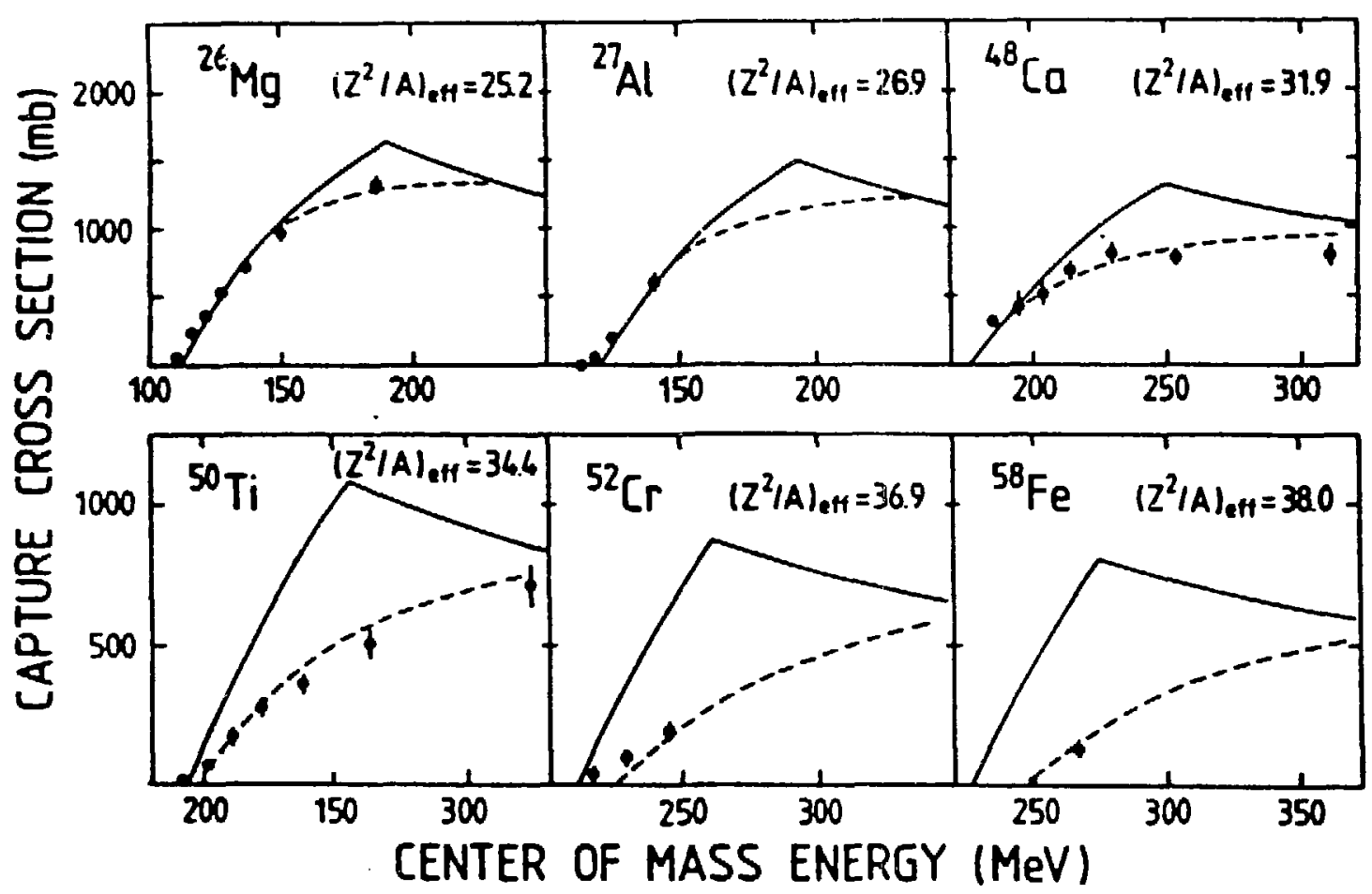

Excitation functions for capture reactlons. The full line is the prediction based on the one-dimensional proximity model of capturing spheres, Refs. 1,4, and 5. As suggested by the analysis in Ref. 1 the sum of the two nuclear radil has been increased by $0.23 \mathrm{fm}$ to obtain normalization with the $\mathrm{Pb}+\mathrm{Mg}$ and $\mathrm{Pb}+\mathrm{Al}$ data. Dashed lines a re predictions of Swiatecki's two-dimensional, extra-push model (Ref. 7) with the fitted values of $a=10$ and $\left(Z^{2} /\right.$ A) ff $^{\text {th }}=32.5$.

Fig 4.5.4. Taken from [SB81]. Reproduced with permission of the American Physical Soclety and the author. 


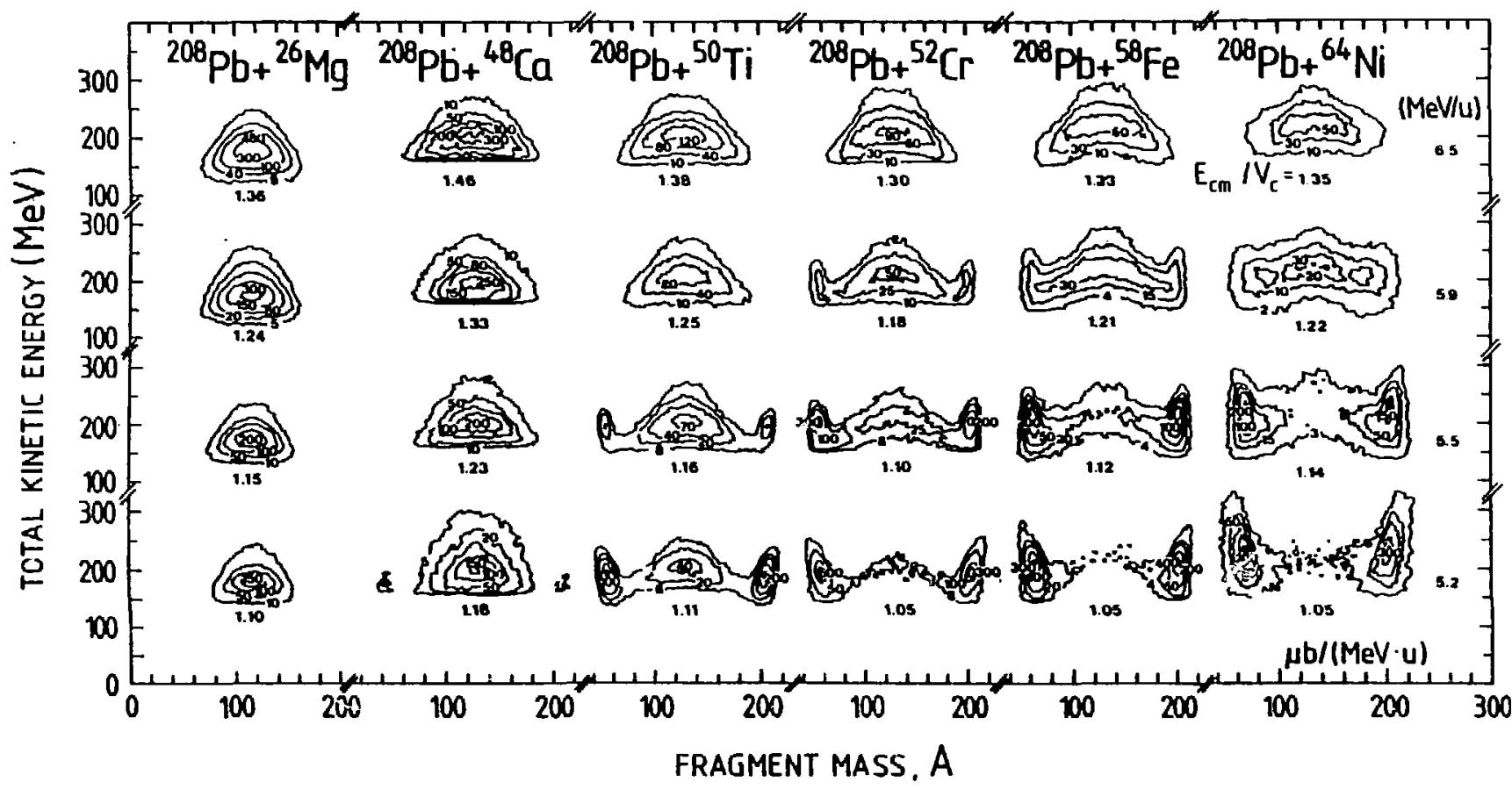

Double differential cross sections $\mathrm{d}^{2} \sigma / \mathrm{dAdTKE}$ for the binary products observed in reactions induced by ${ }^{200} \mathrm{~Pb}$ projectiles on ${ }^{20} \mathrm{Mg}$, ${ }^{40} \mathrm{Ca}$, ${ }^{30} \mathrm{Ti}$, ${ }^{32} \mathrm{Cr}$ ${ }^{34} \mathrm{Fe}$ and ${ }^{64} \mathrm{Ni}$ targets (columns) at incident energies of 5.2, 5.5, 5.9 and $6.5 \mathrm{MeV} / \mathrm{u}$ us indicated on the right-fund side of the figure. The cross sections weri obtuined from an integration over the observed ungular range and corrected for the non-abserved region by assuming a $1 /$ sin 0 distribution. The value 0 $E_{\text {c.. }} / V_{\mathrm{c}}$ is indicated below eacli contour plot.

Fig. 4.5.5 Taken from [BC82]. Reproduced with permission of North-Holland Publishing Co. and the author. 
$\left(A_{1}+A_{2}\right) / 2$. For that one has made cuts around $A=90$ and 160 in the double $\therefore$ differential cross section $d^{2} \sigma / d A d E$ shown in fig. 4.5.5.

Figure 4.5 .4 shows that for the reactions ${ }^{208} \mathrm{~Pb}+{ }^{50} \mathrm{~T} 1,{ }^{52} \mathrm{Cr},{ }^{58} \mathrm{Fe}$ the automatic capture does not occur anymore, whereas for ${ }^{208} \mathrm{~Pb}+{ }^{26} \mathrm{Mg}$ the data follow the standard model.

Let us try to understand this in terms of the trajectory model. First consider the potential-energy landscape for symmetric systems where projectile and target are stable. Figure 4.5 .6 shows contour plots of the folding potentials in the $(s, \sigma)$-plane $(\Delta=0)$. The touching point of two spheres $(\alpha=0)$ is denoted by $t$. The barrier Indicated by $b$ is about 0.6 fm before touching. The numbers on the contour lines are energles in MeV with respect to the compound sphere. In going to heavier compound systems we notice that the barrier becomes lower with respect to the energy of the compound sphere. An even more striking feature is the change in the fission saddle position. It moves for heavier systens towards more compact shapes and becomes rather low in energy. The corresponding saddle point shapes are drawn in the lower right-hand corner. The barrier against fission in this two-dimensional landscape is drastically weakened; in going from $A=188$ to $A=246$, it decreases from $13.5 \mathrm{MeV}$ down to $2.5 \mathrm{MeV}$. A measure of the fissility is given by the parameter $\zeta=z^{2} / A$ which characterizes the ratio of electric to nuclear forces in the system; 1t changes from 35.8 to 40.7 . Also shown in $\mathrm{FIg.4.5.6}$ are trajectories for head-on collisions. Here, we use the equations of motion (3.6.4)-(3.6.10) where we assume a creeping motion in the neck degree of freedom, $\sigma$. The thick lines are those where we give the system only the center-of-mass energy the two nuclei need to reach the top of the barrler assuming that there is no friction. For $A=188(\zeta=35.8)$ the system comes in along $\sigma \sim 0$ and climbs up the mountain, but does not quite make it to the top, 
since we have a proxialty friction which is taking away kinetic energy. Since we are in a two-dimensional parameter space, the nuclear forces may pull small noses out of the spheres, so that the energy is lowered and the two nuclei come into contact by sneaking around the peak. Therefore, despite the friction, the turning point $(\dot{s}=0)$ happens to be again at about $s=s_{b}$. This might be why the energy loss, which is due to friction before the system gets to the barrier, does not enter in the simple expression (4.5.2). Now the system feels already the strong force pulling downhill towards neck formation. Since the scission saddle appears far to the right irom the top of the barier (more stretched shape), the system ends up in a single-sphere shape; automatic capture has taken place. However, if we take $A=208$ and $\zeta=$ 38.1 , the trajectory runs downhill and comes close to the saddle point, where the decision is made whether 1 t will continue to the left and be captured $n \tau$ proceed to the right and scission. In this case it scissioned. For $A=246, Z=100$ the fission barrier is only $2.5 \mathrm{MeV}$ high and a head on trajectory with an energy equal to the barrier does not even come close to the saddle point. Based on these examples one expects a rather abrupt breakdown of the automatic capture assumption around $\zeta=z^{2} / A=37$. For these symmetric systems capture means the formation of a compound mucleus behind the fission saddle.

Let us now consider the role of the asymmetry of the entrance channel. Figure. 4.5.7 shows the potential energy for three different asymmetries for the same compound nucleus ${ }^{246} \mathrm{Fm}$. First, we notice that the conditional saddle point for fixed asymmetry, $\Delta$, moves towards more stretched shapes for more asymmetric systems. The asymmetry has a similar effect on the relative position between barrier and conditional saddle as the fissility has on the unconditional saddle (c.f. fig. 4.5.6). An effective fissility parameter has been introduced as [BA74, SW81] 

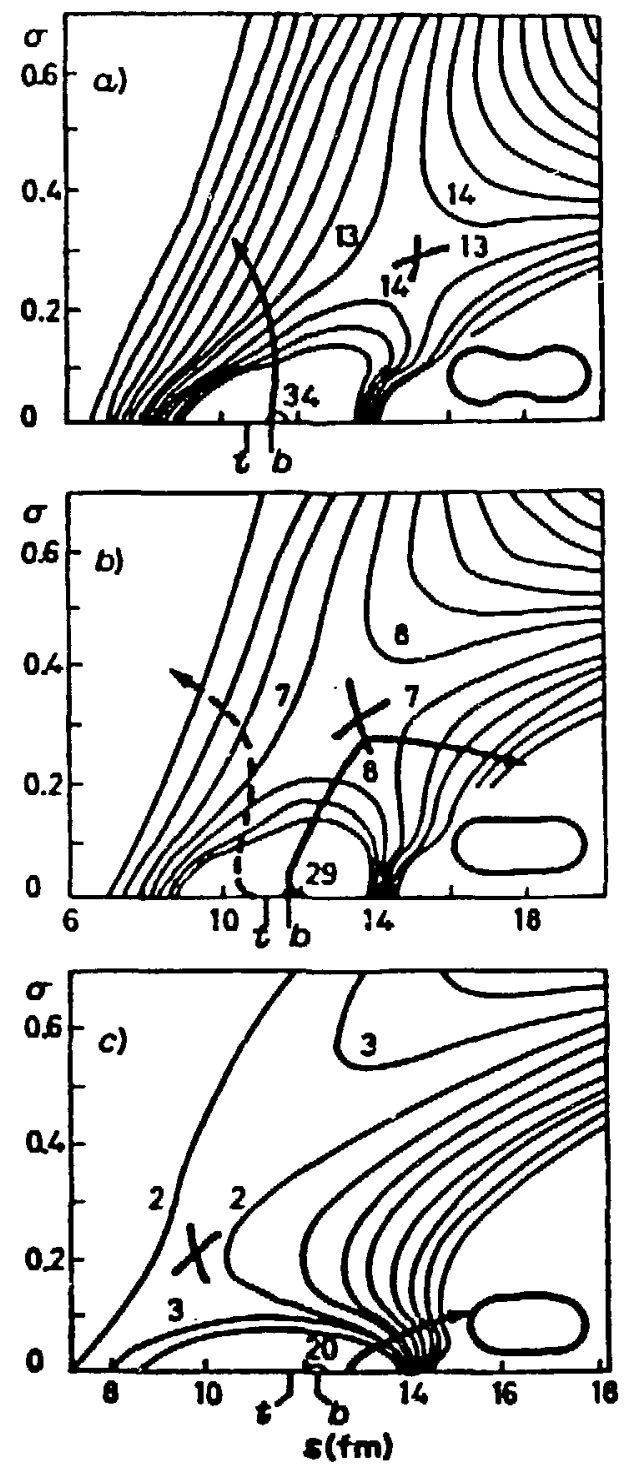

F1g. 4.5.6 Contour maps for the potential energy of symmetric systems and trajectorles for head-on collisions: a) $A=188, Z=$ $\left.\left.82, \zeta=Z^{2} / A=35.8 ; b\right) \quad A=208, Z=89, \zeta=38.1 ; c\right) \quad A=$ 246, $Z=100, \zeta=40.7$. The touching point and the barrier of two spheres are denoted by $t$ and $b$, respectively. The full trajectories start with an intial energy $E_{\mathrm{cm}}$ equal to the barrier energy $V_{b}$. The dashed trajectory starts at the toucising point with zero velocity. In the lower right-hand corners the saddle point shapes are shown. 

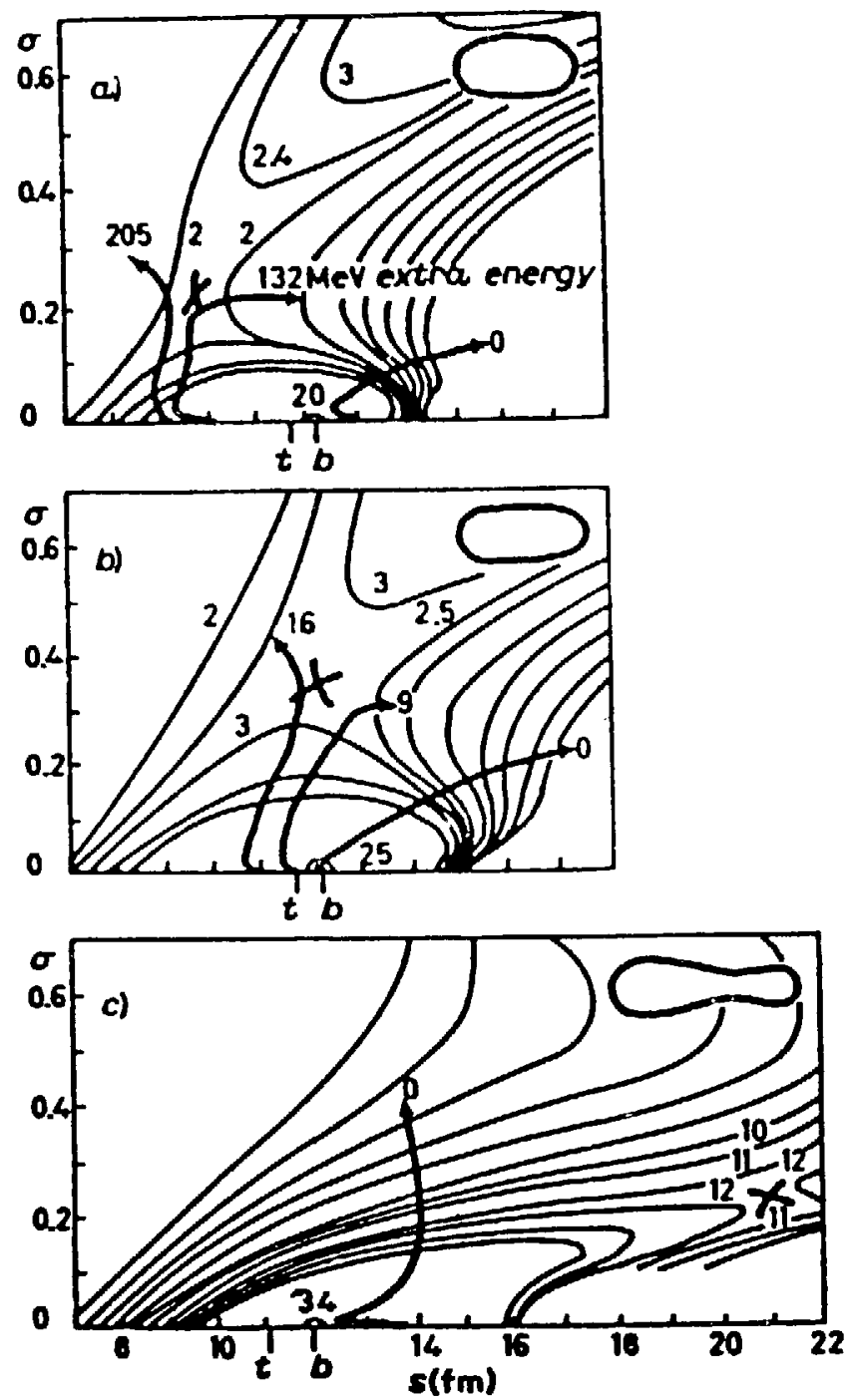

Fig. 4.5.7 Same as fig. 4.5 .6 but for the compound system ${ }^{246} \mathrm{Fm}$ and different asymmetries: a) ${ }^{123} \mathrm{Sn}+123 \mathrm{Sn}, \zeta_{e}=40.7$; b) ${ }^{170} \mathrm{Er}+{ }^{76} \mathrm{Ge}, \zeta_{e}=37.2$; c) ${ }^{206} \mathrm{~Pb}+{ }^{40} \mathrm{Ar}, \zeta_{\mathrm{e}}=28.9$. The head-on trajectories are calculated with different extra energies $\Delta E$ (《<extra push») above the barrier. 


$$
\zeta_{e} \equiv\left(\frac{Z^{2}}{A}\right)_{\text {eff }}=\frac{4 Z_{1} Z_{2}}{A_{1}^{1 / 3} A_{2}^{1 / 3}\left(A_{1}^{1 / 3}+A_{2}^{1 / 3}\right)}
$$

The hope was that asymmetric systems that have the same effective fissility $\zeta_{e}$ should behave similar in the formation of a captured system.

The trajectories included in fig. 4.5.7 have been calculated by keeplng specifically the asymmetry fixed to its initial value in order to see at what c.m. energies they will be captured behind the conditional saddle. Here, the question arises if the conditional saddle has a significance at all. As we have seen in section 4.2 this is the case for necked-in shapes. The strong window friction does not allow sizeable mass drifts in the entrance channel. Therefore, one might call the system captured if it gets caught behind the conditional saddle and cannot reseparate immediately. of course, it is not really caught because the asymmetry degree of freedom relaxes, even if on a long time scale, and the system fissions again. We want to call those events quasifission where the trajectory did not get behind the fission saddle which means the system did not form a compound nucleus. Nevertheless, due to the long reaction time the cross section of the quasifission events may be rather close to a $1 / \sin \theta$ distribution.

In the upper and the central part of fig. 4.5.7 trajectories with an extra energy ebove the barrier are also shown. In the case of ${ }^{170} \mathrm{Er}+$ ${ }^{76} \mathrm{Ge}$ an extra energy of $16 \mathrm{MeV}$ is enough to drive the system behind the conditional saddle point. For ${ }^{123} \mathrm{Sn}+{ }^{123} \mathrm{Sn}$ as much as $205 \mathrm{MeV}$ is needed to get behind the scission saddle. Nix and Sierk discussed this phenomenon already in 1977 [NS77, DS83]. Swlatecki called this energy the "extra push" [SW81, SW82]. 
From a schematic trajectory model Swlateckl developed the "extra push" model which predicts the extra push energy to be quadratic in the effective fissility, $\zeta_{e}$, defined in eq. $(4.5 .4)$

$\Delta E=\quad a^{2} \frac{A_{1}^{1 / 3} A_{2}^{1 / 3}\left(A_{1}^{1 / 3}+A_{2}^{1 / 3}\right)^{2}}{A_{1}+A_{2}} 7.6 \times 10^{-4} \operatorname{MeV}\left(\zeta_{e}-\zeta_{e}^{t h}\right)^{2}$

0

$$
\text { for } \zeta_{e}<\zeta_{e}^{\text {th }}
$$

The steepness a and the threshold $\zeta_{e}^{\text {th }}$ are parameters that have to be determined by using a more realistic model. One can extend formula (4.5.5), which was derived for central collisions, to reactions with angular momentum, by treating the centrifugal force as an enhancement of the Coulomb force. Thus, the effective fissility becomes $\ell$-dependent. For details see [SW82]. In this paper Swiatecki has reproduced successfully the capture cross section of the $\mathrm{Pb}+\mathrm{Mg}, \mathrm{Al}, \mathrm{Ca}, \mathrm{Ti}, \mathrm{Cr}, \mathrm{Fe}, \mathrm{Ni}$ data.

An attempt to verify eq. $(4.5 .5)$ with the model discussed in section 3 has failed. The reason is that for symmetrlc systems there is no way to define a capture cross section in analogy to the highly asymmetric systems because the final mass distribution is centered around the initial masses. There are two sensitive questions which can be answered by the model and by measurements. First, what is the extra energy required to form a compound nucleus in central collisions. Swlatecki called this the "extra extra push". This is a tough question for the experimentalist [SC84] because it 
requires the measurement of the evaporation residues (small cross section) and the real fusion-fission events. In the model one would look at trajectories which get caught behind the fission saddle. The model indicates that for symmetric systems $\Delta E$ rises much faster than the curve deduced from the capture cross section. Going to asymmetric systems the extra extra push curve does not scale according to $\zeta_{e}$.

Second, what is the shape of $\mathrm{d} \sigma / \mathrm{dA}$, and if there is a well pronounced quasi-fission peak, what is the corresponding cross section. Experimentally it has been measured. All models which try to predict capture cross sections without being able to make any prediction about do/dA [GS82, Fr83, Fr84] are not really helpful in understanding the data. The desirable goal should always be to calculate what has been neasured. Here, it means that trajectorles which get caught behind the conditional saddle need not correspond to those events that are included in the experimental capture cross section. In the following section we shall discuss calculations where the asymmetry degree of freedom is allowed to evolve dynamically.

\subsection{Dynamics in the mass asymmetry}

If the beam energy is only little higher than the coulomb barrier and the initial system is rather asymmetric a substantial mass rearrangement has been observed [BC82, TB84]. In $\mathrm{fig}, 4.6 .1$ the double differential cross sections are displayed for two systems. The U+Al case shows a well separated quasi Eission part around symmetric mass division's, whereas the U+Ca case has no pronounced minimum in $d \sigma / d A$ between the Initial mass division and symmetric splitting. The results of the erajectory calculations are a'so included in fig. 4.6.1. For UtAl there is a "jump" in going from an incoming $\ell$-value of $46 \mathrm{~K}$ to $45 \mathrm{~K}$. The final mass number changes rapidly by 43.6 units and the final 


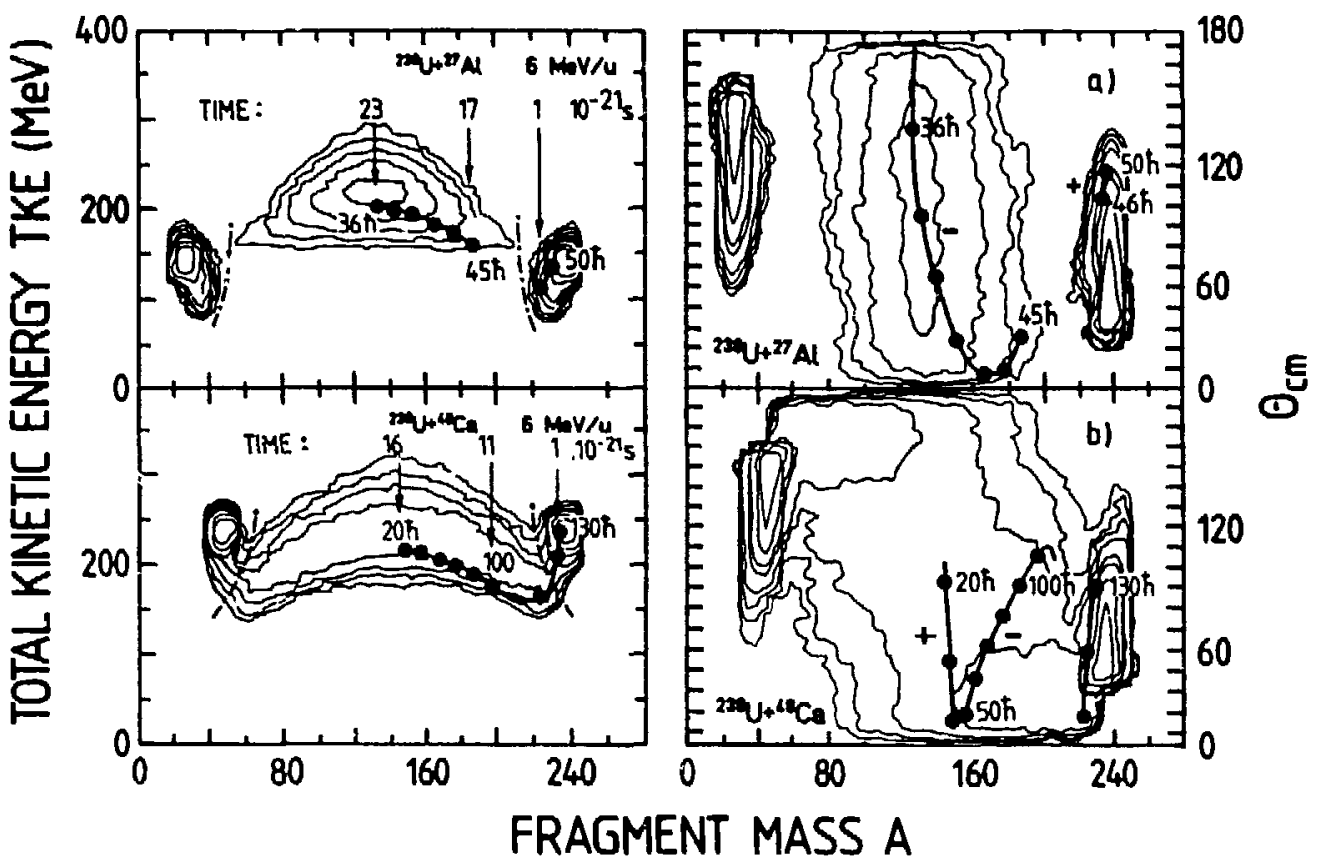

Fig. 4.6.1 Contour plot of $d^{2} \sigma / d A d E$ and $d^{2} \sigma / d A d \theta$ for $U+A l$ and $U+C a$ at $6 \mathrm{MeV} / \mathrm{u}$ taken from [TB84]. The solid line connects the results of the trajectory calculation. On the left hand side three reaction times are given for an incoming $\ell$-value of 36,45 , and 46 $h$ for the $U+A l$ case and $l=20,100$, and $130 k$ for $U+C a$. The plus and minus signs on the r.h.s. Indicate the positive and negative branches of the scattering angle, respectively. Reproduced with permission of the American Physical Society and the author.

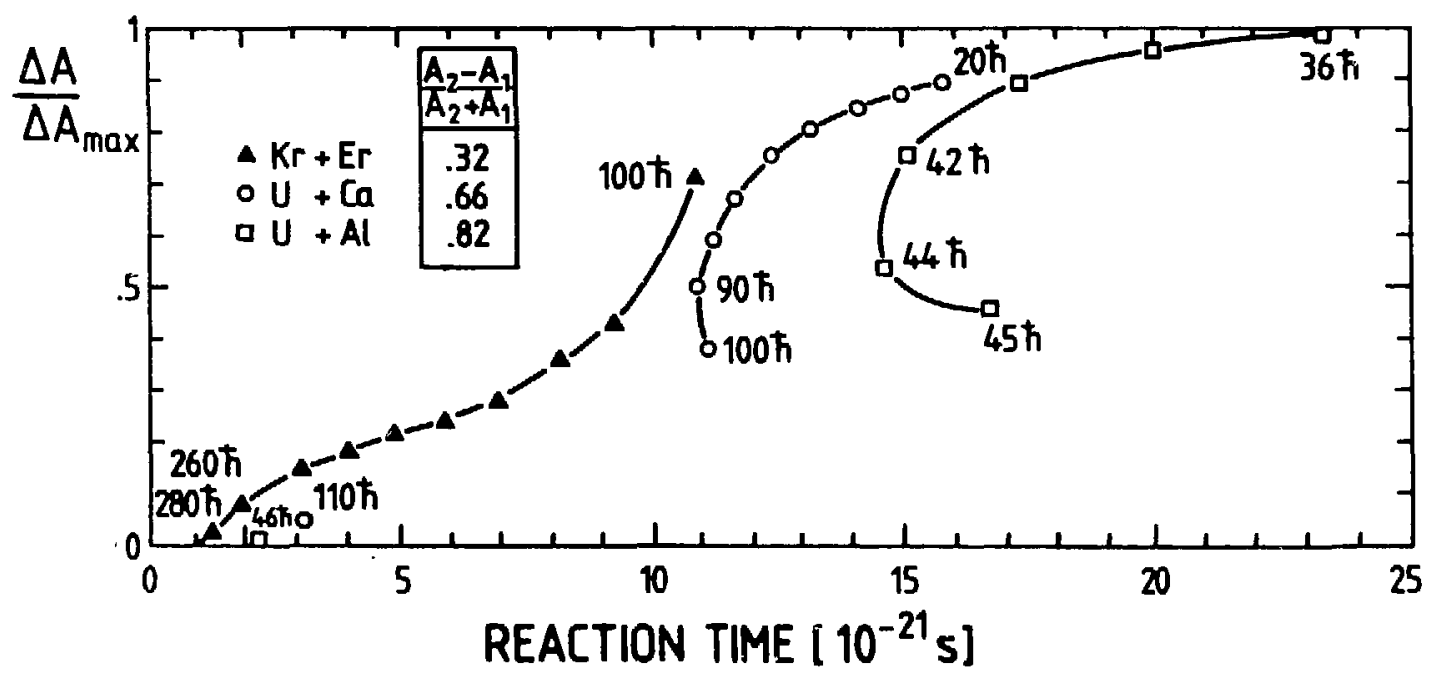

Fig. 4.6.2 Percentage of the possible mass drift $\Delta A / \Delta A$ max as a function of the reaction time. ( $\Delta A=A_{1}-A_{1 f i n a l}, \Delta A_{\max }=A_{1}-\left(A_{1}+A_{2}\right) / 2$ 

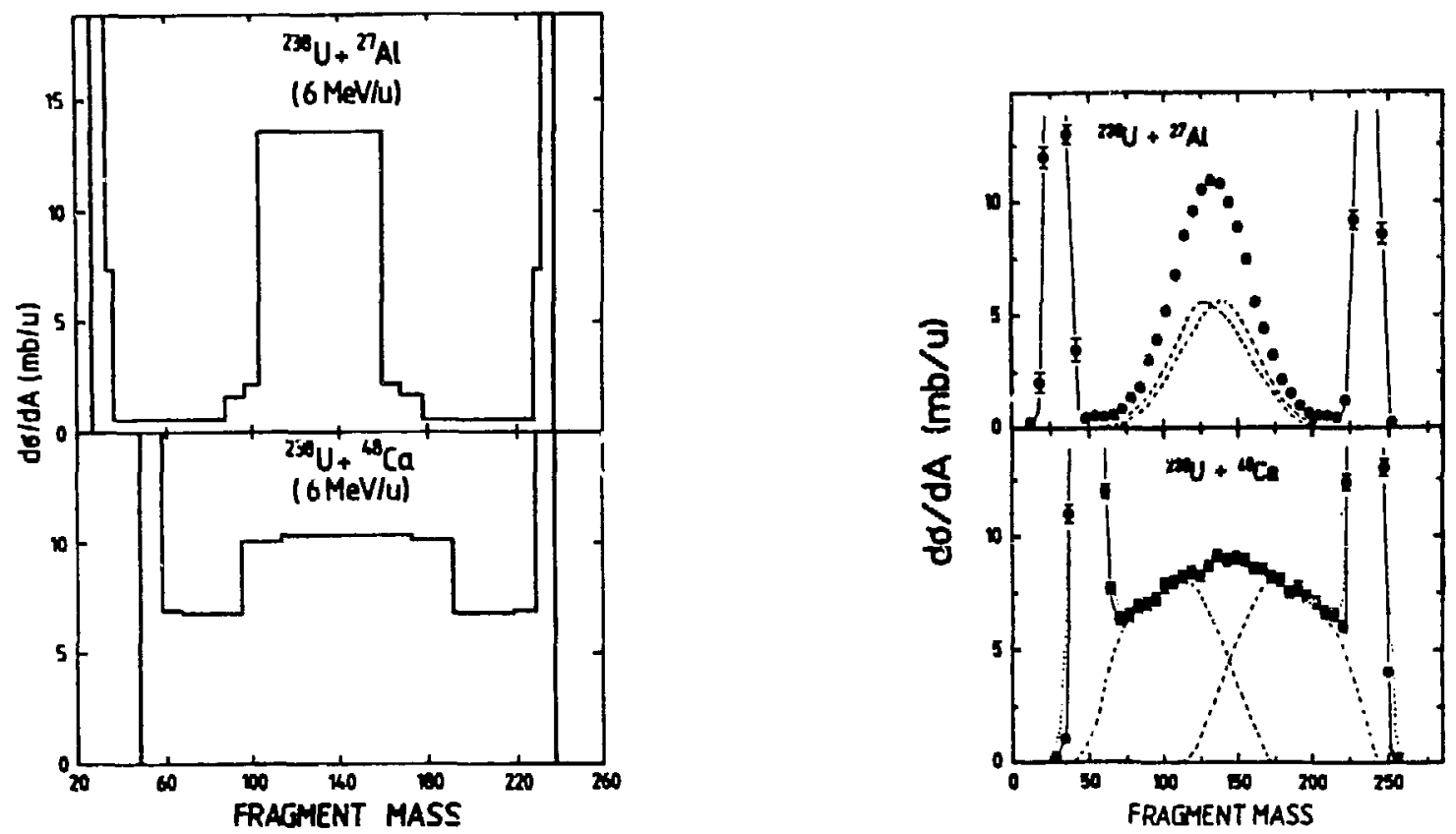

FIg. 4.6.3 do/dA from the trajectory calculation (1.h.s.) compared to the experimental data from [TB84] (r.h.s.).
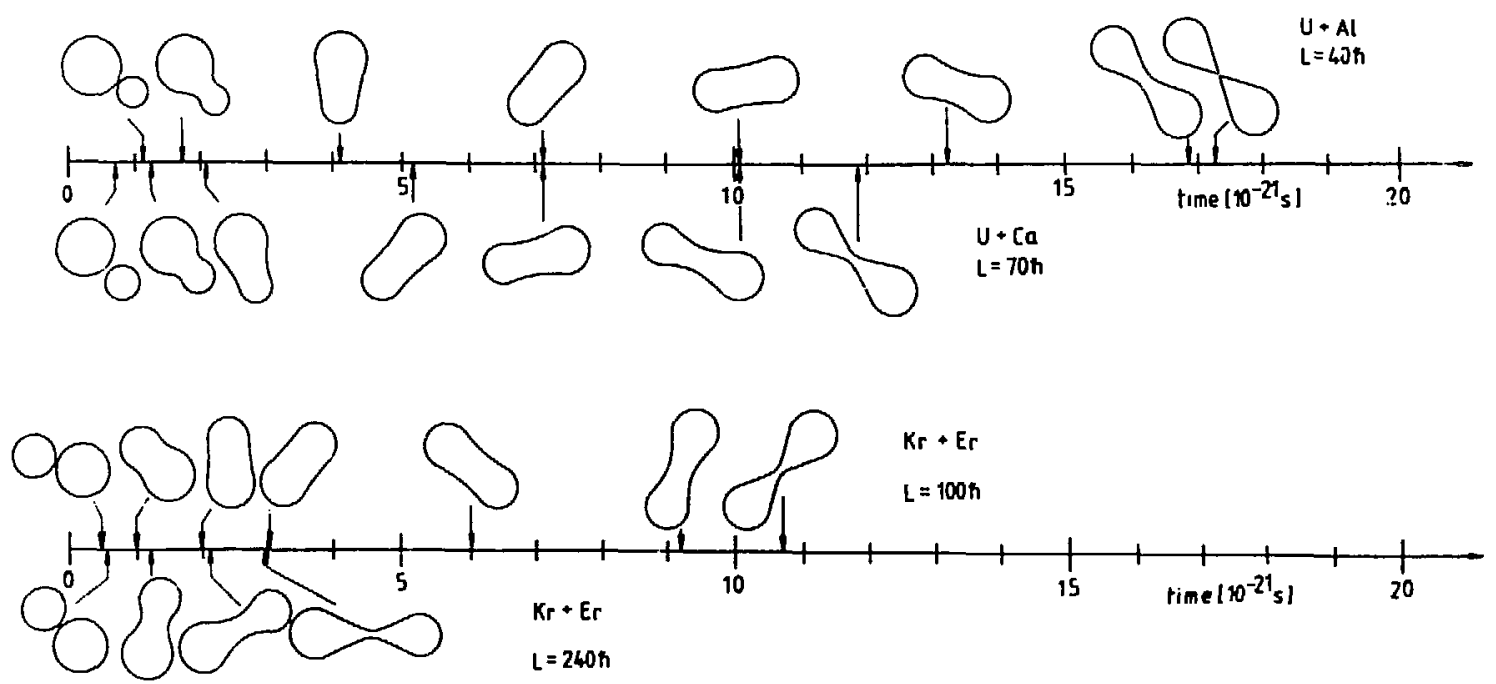

Fig. 4.6.4 Time evolution of the shape for four different trajectorles: $U+\mathrm{Al}$ at $\mathrm{E}_{\mathrm{lab}}=6 \mathrm{MeV} / \mathrm{u}, \ell=40 \mathrm{~h} ; \mathrm{U}+\mathrm{Ca}$ at $\mathrm{E}_{\mathrm{lab}}=6 \mathrm{MeV} / \mathrm{u}$, $\ell=70 \mathrm{~K} ; \mathrm{Kr}^{=} \mathrm{Er}$ at $\mathrm{E}_{1 \mathrm{ab}}=8.2 \mathrm{MeV} / \mathrm{u}, \ell=100 \mathrm{~K}$ and $\ell=240 \mathrm{~K}$. 


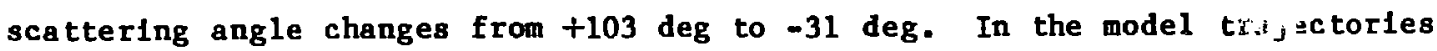
wth $\ell>46 \mathrm{~h}$ do not get behind the condltiona 1 saddle, whereas trajectorles with $\ell<45 \mathrm{~h}$ get caught. They can escape only by changing the mass asymmetry In order to lower the conditional saddle. But this takes a long time, in this case $17 \cdot 10^{-2} 1_{\mathrm{g}}$ (c.f. fig. 4.6 .1 and $\mathrm{fig}, 4.6 .2$ ). In the UtCa case the "jump" occurs between $\ell=110 \mathrm{~h}$ and $\ell=100 \mathrm{~h}$, but $1 \mathrm{f}$ is not as dramatic in the final fragment mass. The cross section $d \sigma / d A$ has been calculated and is compared with the experimental one in $\mathrm{fig} \cdot 4.6 .3$. The two different types of reactions - quasi fission versus more continous mass rearrangement - Is nicely reproduced.

The speed of the mass drift can be recognized in $\mathrm{fig} \cdot 4.6 .2$ where we relate for three reactions the percentage of the possible change in the mass number to the calculated reaction times. The previously discussed "jump" for $\mathrm{U}+\mathrm{Ca}$ and U+Al is quite dramatic.

In $\mathrm{fIg}$. 4.6.4 we show the explicit time dependence of the shape for some of the trajectorles. In the U+Al, $\ell=40 \mathrm{~h}$ trajectory the mass has drjfted $89 \%$ of its way to symmetry. One sees that during the time interval 5 to $10 \cdot 10^{-21} \mathrm{~s}$ the shapes are not of the di-nuclear type. The same is true for the $U+C a, l=$ 70 h case around $5 \cdot 10^{-21} \mathrm{~s}$. The system enters the so called mono-nuclear regime, where the neck has disappeared and the window (which actually cannot be defined reasonably anymore) is widely open. Here, one cannot talk about two nuclei which have more or less kept their identity but rather one deals with a single nucleus where $\Delta=\left(R_{1}-R_{2}\right) /\left(R_{1}+R_{2}\right)$ has become a measure for the octupole moment rather than for the mass asymmetry. Along with the disappearance of the window the window friction has gone. Therefore, we use the wall dissipation alone for mono-nuclear shapes. It turns out that the friction coefficient for the asymmetry degree of freedom is about 4-8 times 
smaller in the mono-nuclear case compared to the di-nuclear case. This means that in the model the asymmetry can relax about 4-8 times faster for mononuclear shapes compared to di-nuclear shapes with fat necks. It is this feature which shortens the reaction times for the U+Ca case compared to UtAl (c.f. fig. 4.6.2). The model seems to predict reaction times that are too long i.e. two negative scattering angles for $\mathrm{U}+\mathrm{Ca}$. This might be due to the crude way of combining window-plus-wall and wall friction by a form factor which as a function of the ratio of neck area to surface area of the smaller nucleus $\left(x=R_{\text {neck }}^{2} / R_{1}^{2}\right)$, turns on and off the different contributions. We assumed to be in a di-nuclear regime if $x<.8$ and to be in the mono-nuclear regime if $x>1$. In between we choose the $\sin ^{2}$ as a smooth interpolation (see eq. $(3 \cdot 4 \cdot 76)$.

A more satisfying way to describe the transition from the di-nuciear to the mono-nuclear regime in the picture of the long-mean-free-path dissipation mechanism is not at our hand. These calculations, however, show that the sensitivity of the reaction time (which shines through in the scattering angle) on the friction coefficient might enable us to learn about the dynamics of the mass asymmetry degree of freedom. Necessary for that are experimental data like those in [TB84] where a rather asymmetric entrance channel leads to a composite system with high fissility. The energy should be high enough to get the nuclear system behind the conditional saddle but not too large in order to keep the angular momentum low. This is necessary to stay away fiom the orbiting type cross section $(\mathrm{d} \sigma / \mathrm{d} \Omega \propto 1 / \mathrm{sin} \theta)$ which is not informative. 


\section{APPENDIX}

\section{The Wigner Function}

There exists a representation of quantum mechanics which deals with expressions quite close to classical mechanics. This remarkable fact was discovered by E. Wigner in 1932. Let us define, and study, the following function called the Wigner function

$$
f(q, p)=\frac{1}{(2 \pi h)^{3}} \int d^{3} s e^{\frac{1}{h} p s} \rho\left(q-\frac{s}{2}, q+\frac{s}{2}\right)
$$

where $q, s$ and $p$ are vectors for position and momentum, respectively, and $\rho\left(q, q^{\prime}\right)$ is the one-body density maxtrix in coordinate respresentation defined as

$$
\rho\left(q, q^{\prime}\right)=\operatorname{Tr}\left(\underset{\Psi^{+}}{\Psi^{\prime}}\left(q^{\prime}\right) \underset{\sim}{\Psi}(q) \underset{\sim}{R}\right)
$$

where $\Psi^{+}$and $\Psi$ are field operators and $\underset{\sim}{\mathrm{R}}$ is the statistical operator (density matrix) describing the system under consideration. Quantum mechanical operators in the Fock space are marked by an underlining twiddle and all traces are to be taken in the Fock space. The first thing to note is that $f(q, p)$ is real. This can be verified easily from $(A .1),(A .2)$ and ${\underset{\sim}{R}}^{+}=\underset{\sim}{R}$. Let us derive a few helpful relations. If a basis set is created by fermion creation operators $a_{\alpha}^{+}$as

$$
\underset{\sim \alpha}{a}|0\rangle=|\alpha\rangle \quad \text { for } \alpha=1,2, \ldots,
$$

then the fleld operators can be written as

$$
\begin{aligned}
& \Psi^{+}(q)=\sum_{\alpha=1}^{\infty} \psi_{\alpha}^{\star}(q) \underset{\sim \alpha}{a_{\alpha}^{+}} \\
& \psi(q)=\sum_{\alpha=1}^{\infty} \psi_{\alpha}(q) \underset{\sim}{a_{\alpha}},
\end{aligned}
$$

where the single-particle wave functions are given by

$$
\psi_{\alpha}(q)=\langle q| \alpha
$$


The unit operator in the one-particle subspace of the Fock-space can be written as

$$
1=\int d^{\prime} q \mid q>\left\langle q\left|=\int d^{3} p\right| p><p|=\Sigma| \alpha>\alpha\right|
$$

The eigenstate $|p\rangle$ and $|q\rangle$ of the momentum and position operator are related by

$$
\langle q \mid p\rangle=\frac{1}{(2 \pi h)^{3 / 2}} e^{\frac{1}{\hbar h} p q}
$$

which yields

$$
\begin{aligned}
& |p\rangle=\int d^{3} q|q\rangle\langle q \mid p\rangle=\frac{1}{(2 \pi h)^{3 / 2}} \int d^{3} q e^{\frac{1}{h} p q}|q\rangle \\
& \left\langle p^{\prime} \mid p\right\rangle=\frac{1}{(2 \pi h)^{3}} \int d^{3} q e^{\frac{1}{h}\left(p-p^{\prime}\right) q}=\delta\left(p-p^{\prime}\right)
\end{aligned}
$$

and

$$
\left\langle q^{\prime} \mid q\right\rangle=\delta\left(q-q^{\prime}\right)
$$

Using relations (A.4) and (A.5), a different representation of the one-body density matrix (defined in $(A, 2)$ ) can be verified easily:

$$
\rho\left(q, q^{\prime}\right)=\sum_{\alpha, \beta} \psi_{\alpha}(q) \rho_{\alpha \beta} \psi_{\beta}^{*}\left(q^{\prime}\right)
$$

where

$$
\rho_{\alpha \beta}=\operatorname{Tr}(\underset{\sim \beta}{a} \underset{\sim \alpha}{a} \underset{\sim}{\mathrm{a}})
$$

Hence, the Wigner function can also be written as

$$
f(q, p)=\frac{1}{(2 \pi /)^{3}} \sum_{\alpha, \beta} \rho_{\alpha \beta} \int d^{3} s e^{\frac{1}{h} p s} \psi_{\alpha}\left(q-\frac{s}{2}\right) \psi_{\beta}^{*}\left(q+\frac{s}{2}\right)
$$

Definition (A.1) and the equivalent ( $A .14$ ) can be inverted:

$$
\rho\left(q, q^{\prime}\right)=\int d^{3} p e^{-\frac{1}{h} p\left(q^{\prime}-q\right)} f\left(\frac{q^{\prime}+q}{2}, p\right)
$$


or

$$
\rho_{\alpha \beta}=\int d^{3} p d^{3} q d^{3} s e^{-\frac{1}{h} p s} \psi_{\alpha}^{\star}\left(q-\frac{s}{2}\right) \psi_{B}\left(q+\frac{s}{2}\right) f(q, p)
$$

Equation (A.15) is obtained by multiplying eq. (A.1) by $e^{-\frac{1}{h} p s^{\prime}}$ and integrating over p. Equation (A.16) is obtaired by using the inversion of eq. $(A .12)$ :

$$
\rho_{\alpha \beta}=\int d^{3} q d^{3} q^{\prime} \psi_{\alpha}^{*}(q) p\left(q, q^{\prime}\right) \psi_{\beta}\left(q^{\prime}\right) \text {, }
$$

and substituting eq. (A.15). These relations imply that the one-body density matrix and the Wigner function are completely equivalent descriptions of the single-particle properties of a given system.

Let us now consider two simple examples. If the statistical operator $\underset{\sim}{R}$ represents a single slater determinant then $\rho_{\alpha \beta}=n_{\alpha} \delta_{\alpha \beta}$ where $n_{\alpha}=0,1$ are the occupation numbers of the single-particle levels. In this case the representation of the Higner function given in eq. (A.14) is most convenient; the summation runs just over the occupled levels.

Another simple example is that of a Fermi gas. Let us denote by $\underset{\sim}{\mathrm{H}}$ the single-particle hamiltonian and by $\underset{\sim}{\mathrm{N}}$ the particle number operator. The statistical operator $\underset{\sim}{R}$ for a Fermi gas is then given by

$$
\underset{\sim}{\mathrm{R}}=\frac{1}{\mathrm{Z}} \mathrm{e}^{-\beta\left(\mathrm{\sim}_{0}-\mu \mathrm{N}\right)}
$$

where $Z$ is the partition sum:

$$
\mathrm{Z}=\operatorname{Tr}\left(\mathrm{e}^{-\beta\left(\stackrel{\mathrm{H}}{0}_{0}^{-\mu N}\right)}\right)
$$

$\beta$ is the inverse temperature $(\beta=1 / T)$ and $\mu$ is the chemical potential. By choosing the appropriate single-particle basis, $\underset{\sim}{\mathrm{H}}$ and $\underset{\sim}{\mathbb{N}}$ take the form

$$
\begin{aligned}
& \stackrel{\mathrm{H}}{\sim}_{0}=\Sigma \varepsilon_{\alpha} \stackrel{\mathrm{a}}{\sim \alpha}_{\alpha}^{\mathrm{a}} \underset{\alpha \alpha}{\mathrm{a}} \\
& \underset{\sim}{\mathbb{N}}=\sum_{\alpha} \underset{\sim \alpha}{\stackrel{a}{\sim}} \underset{\sim \alpha}{a}
\end{aligned}
$$

which can be used to evaluate the one-body density matrix: 


$$
\rho_{\alpha \beta}=T_{r}\left(a_{\beta}^{+} \stackrel{\sim}{\alpha}_{\alpha} \underset{\sim}{R}\right)=\frac{\delta_{\alpha \beta}}{1+\exp \left(\beta\left(\epsilon_{\alpha}-\mu\right)\right)}
$$

Substituting eq. (A.22) In eq. (A.14) one obtains the Wigner function for a Fermi gas.

A further simplification is obtained by considering an infinitely extended gas. Here, all the knowledge about the system is contained in the occupation nimber $\mathrm{n}(\mathrm{p})$ for a plane wave with momentum $\mathrm{p}$. Since the plane waves also form the elgenstates of the one-body density, we can write

$$
\begin{aligned}
\rho\left(q, q^{\prime}\right) & =\left\langle q|q| q^{\prime}\right\rangle=\int d^{3} p\langle q \mid p\rangle n(p)\left\langle p \mid q^{\prime}\right\rangle \\
& =\frac{1}{(2 \pi h)^{3}} \int d^{3} p e^{-\frac{1}{h} p\left(q^{\prime}-q\right)} n(p)
\end{aligned}
$$

Using definition (A.1) gives us the Wigner function for an Infinitely extended sys tem

$$
f(q, p)=\frac{1}{(2 \pi h)^{3}} n(p)
$$

As expected, the Wigner function does not depend on $q$ anymore. If we are dealing with a thermally equilibrated, Ideal FermI gas n(p) is just the FermI distribution whlch means

$$
f(q, p)=\frac{1}{(2 \pi h)^{3}} \frac{1}{1+\exp \left\{\beta\left(\frac{p 2}{2 m}-\mu\right)\right\}}
$$

The next step of sophistication would be to use the Thomas-Fermi approximation, which should be valid when the potential U varfes slowly in coordinate space, 1.e. $|\partial U / \partial q|<k_{F}$ where $k_{F}=(2 m \mu)^{1 / 2} / h$ is the Fermi wave number and $m$ the mass of a fermion. $I_{1} \cdot \cdots$, one replaces the chemical potential $\mu$ In (A.25) by a coordinate dependent one, namely, $\mu(q)=\varepsilon_{F}-U(q)$, (c.f. fIg. Ai) 


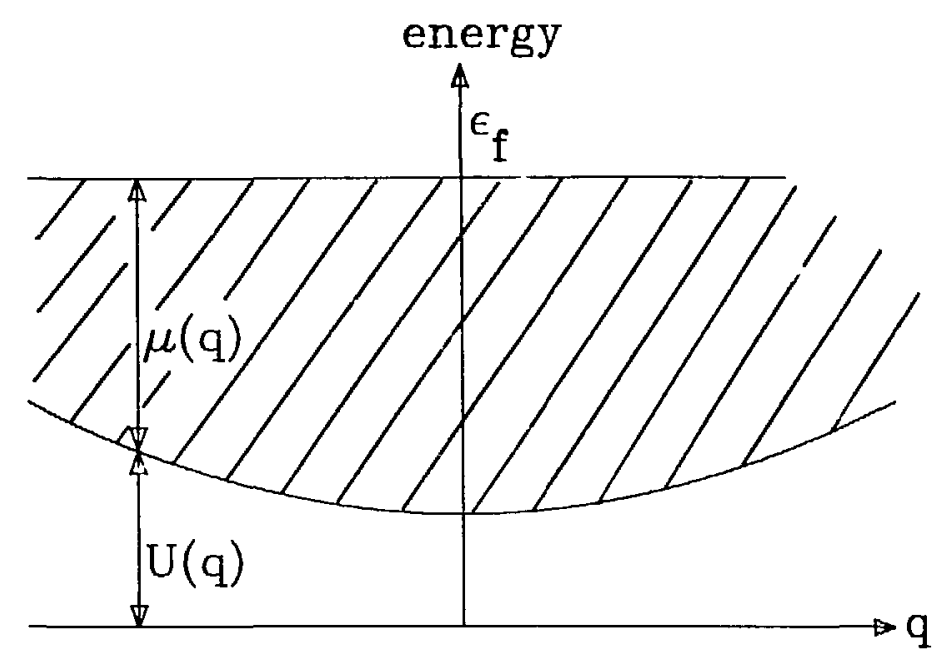

Fig. Al Thomas-Fermi Approximation

It is important to note that the Wigner function not only looks very much like the classical one-body phase distribution but even more prominent is the fact that expectation values of one-body operators can be evaluated with the Wigner function almost as if they were classical objects.

Any one-body operator $\underset{\sim}{\sigma}$ can be written as

$$
\underset{\sim}{\sigma}=\underset{\alpha \beta}{\sum} A_{\alpha \beta} \stackrel{+}{\underset{\sim}{a} \alpha} \stackrel{\mathrm{a}}{\sim} \alpha
$$

Its expectation value is

$$
\begin{aligned}
& \left\langle\underset{\sim}{\sigma}=\operatorname{Tr}(\underset{\sim R}{g R})=\underset{\alpha \beta}{\Sigma} A_{\alpha \beta} \operatorname{Tr}\left({\underset{\sim \alpha}{a}}_{\sim \beta}^{+} \underset{\sim}{\mathrm{a}}\right)\right. \\
& =\sum_{\alpha \beta} A_{\alpha \beta} P_{B \alpha}=\operatorname{Tr}(\underset{\sim}{A} P)
\end{aligned}
$$

Thus, all information needed to calculate the expectation value of an arbitrary one-body operator is the one-body density matrix, or 1 ts equivalence, the Wigner function. Let us first consider the special cases where $g$ is a function of the momentum or the position operator only. Assume 


$$
g=\int d^{3} q \sigma(q) \psi^{+}(q) \psi(q)
$$

where $\sigma(q)$ is a function of $q$ (not an operator), for example, the potentisl energy. Then

$$
\left\langle\underset{\sim}{\langle}=\int d^{3} q \sigma(q) \operatorname{Tr}\left(\Psi^{+}(q) \psi(q)\right)=\int d^{3} q \sigma(q) \rho(q, q)\right.
$$

If we use eq. (A.15) for $q^{\prime}-q=0$ we obtain

$$
\left\langle\underset{\sim}{\sim}=\int d^{3} q d^{3} p f(q, p) \sigma(q)\right.
$$

If $\underset{\sim}{\sigma}$ is a function of momentum only, like the kinetic energy, the expectation value can be expressed as

$$
\begin{aligned}
\langle g\rangle & =\int d^{3} p \sigma(p)\langle p|Q| p\rangle= \\
& =\int d^{3} p \sigma(p) \int d^{3} q d^{3} q^{\prime} \frac{1}{(2 \pi h)^{3}} e^{\frac{1}{h} p\left(q^{\prime}-q\right)} \rho\left(q, q^{\prime}\right)
\end{aligned}
$$

which, by a change of variables $q^{\prime}-q+s$ and $\left(q+q^{\prime}\right) / 2+q$ becomes

$$
\langle q\rangle=\int d^{3} q d^{3} p f(q, p) \sigma(p)
$$

From eqs. (A.30) and (A.32) it is clear that the expectation values are calculated in the same fashion as in classical mechanics where $f(q, p)$ represents the one-body phase space distribution. Note that on the right hand side there are no operator valued quantities anymore.

For an operator containing combinacions of $p$ and $q$ like $p_{\sim}^{2} q$ the situation is somewhat more complicated because they do not commute. H. Weyl postulated the following correspondence principle: if the classical function $\sigma(q, p)$ can be written $a s$

$$
\sigma(q, p)=\int d k d s \Omega(k, s) e^{j(k q+s p)}
$$

then the corresponding quantum operator is

$$
g(q, R)=\int \mathrm{dkds} \Omega(k, s) e^{i(k g+s R)}
$$


Please note that any power $\mathrm{q}^{\mathrm{n}} \mathrm{R}^{\mathrm{m}}$ is still meant to be a one-body operator. The notation is inconsistent since, for example, gq, as it is defined with the field operators, already contains a two-body operator. Therefore, we write $\mathrm{q}_{\mathrm{n}}^{\mathrm{n}} \mathrm{m}$.

For example:

$$
\begin{aligned}
& \sigma(q, p)=q+\Omega(k, s)=1 \nabla \delta(k) \delta(s) \\
& \sigma(q, p)=p+\Omega(k, s)=1 \delta(k) \nabla \delta(s) \\
& \sigma(q, p)=q^{2} p-\Omega(k, s)=\left[1^{2} \nabla^{2} \delta(k)\right] 1[\nabla \delta(s)]
\end{aligned}
$$

where $\nabla \delta(k)$ is the gradient of the delta function which has to be removed by integrating by parts. The resulting operators are:

$$
\begin{aligned}
q & \rightarrow q \\
p & +p \\
q^{2} p & +\frac{1}{3}\left(q_{\sim}^{2} p+q p q+p q^{2}\right)
\end{aligned}
$$

The postulate (A.33) fits right into the scheme of the Wigner function because it implies

$$
\langle\underset{\sim}{g}(R, q)\rangle=\int d^{3} q d^{3} p f(q, p) \sigma(p, q)
$$

This means that the expectation value of any operator is of the same form as the classical expectation value.

Relation (A.36) can be proven by first verifying

$$
\left\langle e^{1(k q+s p)}>=\int d^{3} q d^{3} p f(q, p) e^{i(k q+s p)}\right.
$$

In dofing this, one uses the Identity

$$
e^{(\underset{\sim}{A}+B)}=e^{A} e^{\stackrel{B}{\sim}} e^{-\frac{1}{2}[\underset{\sim}{A}, \underset{\sim}{B}]} \text { if }[\underset{\sim}{A}, \underset{\sim}{B}]=c \text {-number }
$$

and some coordinate transformations. The step to (A.36) is then straightforward. 
In sumnary, the Wigner function is the quantum analogue of the classical single-particle phase space distribution. It is real but not necessarily positive. Nevertheless, any expectation value is calculated as in classical mechanics by integrating over $q$ and $p$. On the other hand, any measurement cannot test the Wigner function in cells of phase space with a volume less than about $h^{3}$. Therefore, if we integrate the Wigner function over a part of the phase space which is compatible with the uncertainty relation we should obtain a positive number. 


\section{References}

BA74 R. Bass, Nuc1. Phys. A231, 45 (1974).

BB78 J. Blocki, Y. Boneh, J. R. Nix, J. Randrup, M. Robe1, A. J. Sierk and W. J. Swiatecki, Ann. Phys. (N.Y.), 113, 330 (1978).

BC8 2 R. Bock, Y. T. Chu, M. Dakowski, A. Gobbi, E. Grosse, A. Olmi, H. Sann, D. Schwalm, U. Lynen, W. Müller, S. Bjornholm, H. Esbensen, W. Wölfi and E. Mnrenzoni, Nucl. Phys. A388, 334 (1982).

BF83 W. Bohne, P. Fröbrich, K. Grabisch, K. Hartmann, H. Lehr, H. Morgenstern and W. Stöffler, Z. Phys. A313, 19 (1983).

BG78 M. Berlanger, P. Grange, H. Hofmann, C. Ng 6 and J. Richert, Phys. Rev. C 17, 1495 (1978).

B180 J. Block1, preprint (Institute of Nuclear Research, Swierk, Poland) INR 1980.

BM83 H. Breuer, A. C. Mignerey, V. E. Viola, K. L. Wolf, J. R. Birkelund, D. Hilscher, J. R. Huizenga, W. U. Schroeder and W. W. Wilcke, Phys. Rer. C 28, 1080 (1983).

BN78 M. Berlanger, C. Ng6, P. Grange, J. Richert and H. Hofmann, Z. Physik A284, 61 (1978).

BR8 2 U. Brosa and W. Cassing, Z. Physik A307, 167 (1982).

BS82 J. Block1 and W. J. Swlateck1, report LBL-12811 (1982).

BT79 J. R. Blrkelund, L. E. Tubbs, J. R. Hulzenga, J. N. De and D. Sperber, Phys. Rep. 56, 107 (1979).

BY8 3 H. Brever, N. R. Yoder, A. C. Mignerey, V. E. Viola and $\mathrm{K}$. Kwla tkowsk1, Nucl. Instr. and Meth. 204, 419 (1983).

CB83 J. Carter, C. Brendel, A. Richter, G. Schneider, H. Feldmeier, W. Bohne, K. Grabisch, H. Lehr and H. Morgenstern, Z. Phys. A313, 57 (i983).

CH43 S. Chandrasekhar, Rev. Mod, Phys. 15, 1 (1943).

CJ78 B, Cauvin, R. C. Jared, P. Russo, R. P. Schmitt, R. Babinet and L. G. Moretto, Nucl. Phys. A301, 511 (1978).

CK.81 W. Cassing and J. Knoll, Phys. Lett. 106B, 443 (1981).

DS76 K. T. R. Davies, A. J. Sierk and J. R. N1x, Phys. Rev. C 13, 2385 (1976).

ES83 K. T. R. Davies, A. J. Sierk and J, R. Nix, Phys. Rev, C 28, 679 (1983). 
EG78 Y. Eyal, A. Gavron, I. Tserruya, Z. Fraenkel, Y. Eisen, S. Wald, R. Bass, G. R. Gould, G. Kreyling, R. Renfordt, K. Stelzer, R. Zitzmann, A. Gobbi, U. Lynen, H. Stelzer, I. Rode and R. Bock; Phys. Rev. Lett. 41, 625 (1978) and Phys. Rev. C 20, 1377 (1980).

Fe84 H. Feldmeler, Dynamics of Heavy-Ion Reactions, in Nuclear Structure and Heavy Ion Dynamics; ed. L. Moretto and R. A. Ricci (North-Holland 1984), p. 274 (ISBN 0-444-86826-7).

FL84 H. Feldmeler, Proceedings of the Workshop on Nuclear Dynamics III, March 5-9, 1984, Copper Mountain, CO; ed. V. Viola, Indiana Nuclear Chemistry Report INC-40007-24.

FK84

H. Frelesleben and J. V. Kratz, Phys. Rep. 106, 1 (1984).

Fr83

Fr84

FS83

FS84

FS8 5

GB84

GG84

GH81

GN80

Gr75

Gr82

GS8 2

HB79

HS77

Ke64

K146

KN79

P. Fröbrtch, Phys. Lett. 122B, 338 (1983).

P. Fröbrich, Phys. Rep. 116, 337 (1984).

F. Fröbrı́ch, B. Strack and M. Durand, Nucl. Phys. A406, 557 (1983).

H. Feldmeler and H. Spangenberger, Nuc1. Phys. A428, 223c (1984).

H. Feldmeier and H. Spangenberger, Nucl. Phys. A435, 229 (1985).

J. J. Griffin and W. Broniowsk1, Nuc1. Phys. A428, 145c (1984).

G. Guarino et al., Nucl. Phys. A424, 157 (1984).

D. H. E. Gross and K. M. Hartmann, Phys. Rev. C 24, 2526 (1981).

A. Gobbi and W. Nörenberg, in Heavy Ion Collisions Vol. 2, ed. R. Bock, North-Holland (1980).

D. H. E. Gross, Nucl. Phy". A240, 472 (1975).

H. Grabert: Projection Operator Techniques in Non-equilibrium Mechanics, Springer Tracts in Modern Physics, Vol. 95, (1982), Springer-Verlag.

D. H. E. Gross and L. Satpathy, Phys. Lett. 110B, 31 (1982).

D. Hilscher, J. R. Birkeiund, A. D. Hoover, W. U. Schröder, W. W. Wilcke, J. R. Huizenga, A. C. Mignerey, K. L. Wolf, H. F. Breuer and V. E. Viola, Jr., Phys. Rev. C 20,576 (1979).

H. Hofmann and P. Siemens, Nucl. Phys. A275, 464 (1977).

I. Kelson, Phys. Rev. 136, B1667 (1964).

J. G. Kirkwood, J. Chem. Phys. 14, 180 (1946).

H. J. Krappe, J. R. Nix and A. J. Slerk, Phys. Rev. C 20, 992 (1979). 
LR63 J. L. Lebowitz and P. Resibols, Phys. Rev. 139, A1101 (1965).

LV85 D. K. Lock, R. Vandenbosch and J. Randrup, preprint: Transport and Evaporation Model Calculation of $\mathrm{N}$ and $\mathrm{Z}$ Distributions for Damped Nucleus Reactions.

MN76 T. Möller and J. R. N1x, Nucl. Phys. A272, 502 (1976).

MN81 P. Möller and J. R. N1x, Nucl. Phys. A361, 117 (1981).

Mo83 L. G. Moretto, Z. Physik A310, 61 (1983) and Proc. Int. Conf. Nucl. Phys., Florence 1983, Vol. II, p. 385.

Ni69

J. R. Nix, Nucl. Phys. Al30, 241 (1969).

$\mathrm{Ni} 84$

J. R. Nix and A. J. Slerk, Nucl. Phy-s. A428, L6lc (1984).

Nö80

W. Nörenberg, in Heavy Ion Collisions Vol. 2, ed, R. Bock, North Holland (1980).

Nö81 W. Nörenberg, Phys. Letters 104B, 107 (1981).

Nö83 W. Nörenberg, Nucl, Phys. A400, 275c (1983) and Nucl. Phys. A409, 191c (1983).

NS77 J. R. Nix and A. J. Slerk, Phys. Rev. C 15, 2072 (1977).

NS80 J. R. NIx and A. J. Sierk, Proc. Int. Workshop on Gross Properties of Nuclel and Nuclear Excltations IX, HIrschegg, Austrla, ed. H.

Feldmeier (1980), p. 74 .

Ra78 J. Randrup, Ann. Phys. 112, 356 (1978).

Ra78a J. Randrup, Nuc 1. Phys. A307, 319 (1978).

Ra79 J. Randrup, Nucl. Phys. A327, 490 (1979).

Ra82 J. Randrup, Nuc 1. Phys. A383, 468 (1982).

Re65 F. Reif, Fundamentals of Statistical and Thermal Physics, McGraw-Hill Kogakusha Ltd., ed. E. U. Condon, Tokyo 1965, p. $560 \mathrm{ff.}$

RN80 S. Riedel and G. Wolschin, Z. Physik A294, 17 (1980).

RS84 J. Randrup and W. J. Swiateckl, Nucl. Phys. A429, 105 (1984).

RW79 C. Riedel, G. Wolschin and W. Nb̈renberg, Z. Phys. A290, 47 (1979).

SB81 H. Sann, R. Bock, Y. T. Chu, A. Gobbi, A. 01mi, U. Lynen, W. Müller,

S. Bjornholm and H. Esbensen, Phys. Rev. Lett. 47, 1248 (1981).

SC84 C. C. Sahm, H. G. Clerc, K. H. Schmidt, W. Reisdorf, P. Armbruster, F. P. Hessberger, J. G. Keller and G. Münzenberg, Z. Physik A319, 113 (1984). 
SH84 W. U. Schröder and J. R. Hulzenga, in Treatise on Heavy Ion Science, ed. D. A. Bromley, Vol. 2, Plenum Press New York and London 1984.

SS81 S. K. Samaddar, D. Sperber, M. Zielinska-Pfabe, M. I. Sobel and S. I. A. Garpman, Phys. Rev. C 23, 760 (1981).

Sw80 W. J. Swiatecki, in Progress in Particle and Nuclear Physics, Vol. 4, edited by D. Wilkinson (1980), p. 383 .

Sw81 W. J. Swiatecki, Physica Scripta 24, 113 (1981).

Sw82 V. J. Swiateck1, Nuc1. Phys. A376, 275 (1982).

TB84 J. Töke, R. Bock, G. X. Dai, S. Gralla, A. Gobbi, K. D. Hildenbrand, J. Kuzminski, W. F. J. Müller, A. Olmi, H. Stelzer, B. B. Back, S. Bjornholm, preprint GSI-84-51 and Phys. Lett. 142B, 258 (1984).

TC79 B. Tamain, R. Chechik, H. Fuchs, F. Hanappe, M. Morjean, C. Ng $6, \mathrm{~J}$. Peter, M. Dakowski, B. Lucas, C. Mazur, M. Ribrag and C. Signarbieux, Nuc1. Phys. A330, 253 (1979).

VL84 R. Vandenbosch, A. Lazzarini, D. Leach, D.-K. Lock, A. Ray and A. Seans ter, Phys. Rev. Lett. 52, 1964 (1984).

We80 $H$. Weldenmüller, in Progress in Particle and Nuclear Physics, Vol. 3, ed. D. Wilkinson (1980) p. 49.

WU45 M. C. Wang and G. E. Uhlenbeck, Rev. Mod. Phys. 17, 323 (1945).

WW8 1 H. J. Wollersheim, W. W. Wilcke, J. R. Birkelund, J. R. Huizenga, W. U. Schröder, H. Frelesleben and D. Hilscher, Phys. Rev. C 24, 2114 (1981).

YN82 H. L. Yadis and W. Nörenberg, Phys. Lett. 115B, 179 (1982).

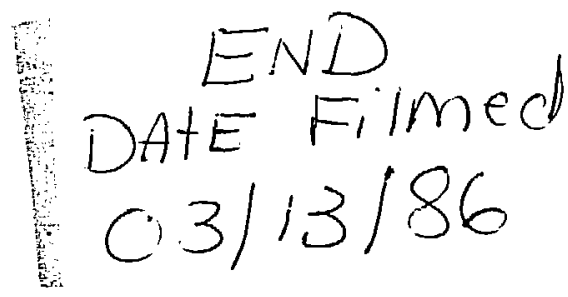

